\title{
NOISE REDUCTION FOR VENTILATION SYSTEMS WITH HEAT EXCHANGERS
}

Mohammad Kojourimanesh 
Graduation committee members:

\section{Chairman and Director of PDEng program}

Prof.dr.ir. G. Brem, University of Twente

\section{Supervisors}

Prof.dr.ir. A. de Boer, University of Twente

Dr.ir. A.P. Berkhoff, University of Twente

\section{Members}

Prof.dr.ir. G. Brem, University of Twente

Prof.dr.ir. A. de Boer, University of Twente

Dr.ir. A.P. Berkhoff, University of Twente

Dr.ir. Y.H. Wijnant, University of Twente

Ir. I. Cleine, Development Engineer R\&D at Brink Climate Systems

The work described in this thesis was performed at the research group Structural Dynamics, Acoustics Control (SDAC), Faculty of Engineering Technology, University of Twente, Enschede, The Netherlands.

This research was carried out with the financial support of the Brink Climate Systems Company.

Mohammad Kojourimanesh

Noise reduction for ventilation systems with heat exchangers

PDEng Thesis, University of Twente, Enschede, The Netherlands

May 2018

ISBN: 978-94-6233-964-4

Copyright (C) by Mohammad Kojourimanesh, Enschede, The Netherlands, 2018

Cover design by Mohammad Kojourimanesh

Printed by Gildeprint, Enschede, The Netherlands 


\title{
NOISE REDUCTION FOR VENTILATION SYSTEMS WITH HEAT EXCHANGERS
}

\author{
PDEng Thesis \\ to obtain the degree of \\ Professional Doctorate in Engineering (PDEng) at the University of Twente, \\ on the authority of the rector magnificus, \\ Prof.dr. T.T.M. Palstra, \\ on account of the decision of the graduation committee, \\ to be defended \\ on Monday the 14th of May 2018 at 13:30 hours
}

by

Mohammad Kojourimanesh
born in 1985
in Shiraz, Islamic Republic of Iran 
This PDEng Thesis has been approved by:

Thesis Supervisor:

Prof.dr.ir. A. de Boer

Co-supervisor(s):

Dr.ir. A.P. Berkhoff 


\section{Contents}

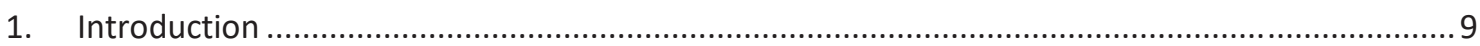

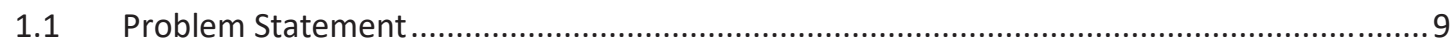

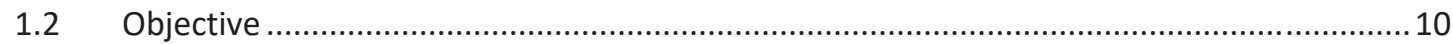

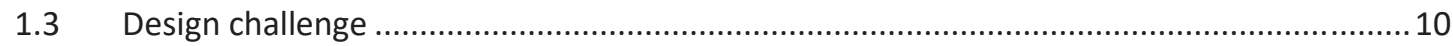

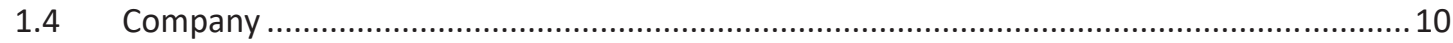

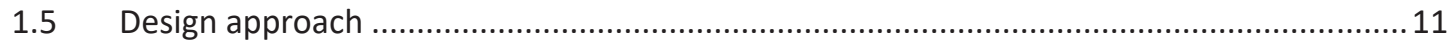

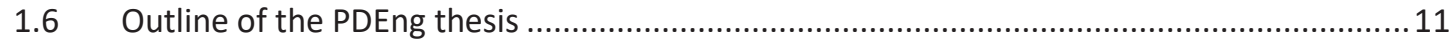

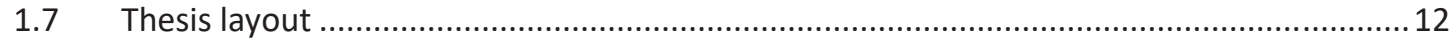

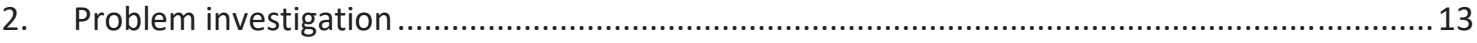

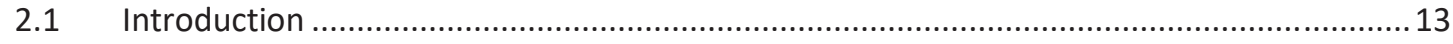

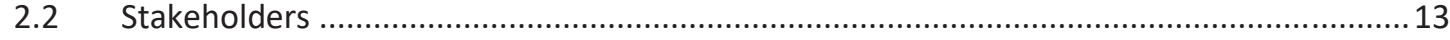

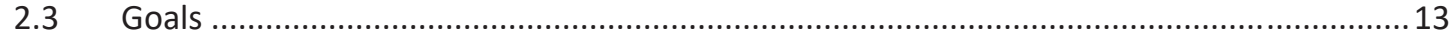

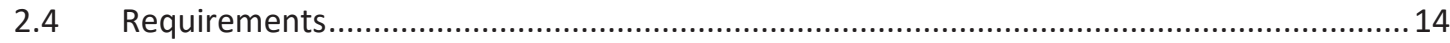

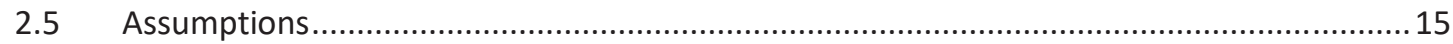

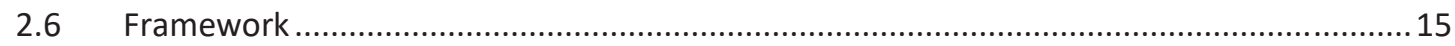

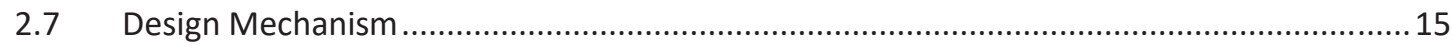

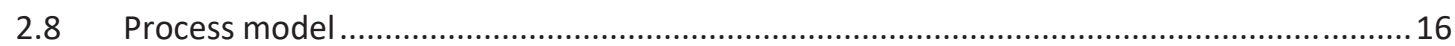

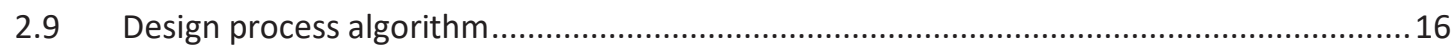

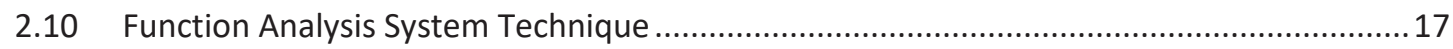

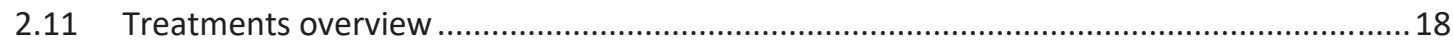

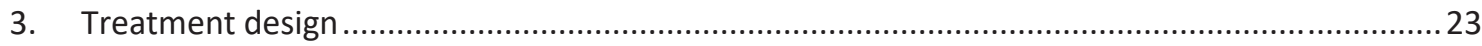

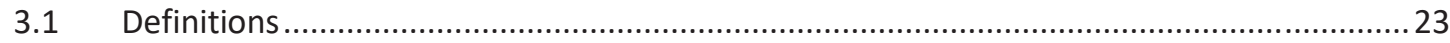

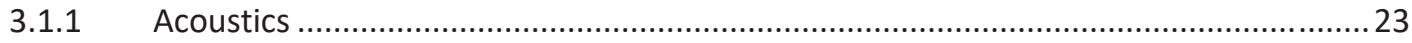

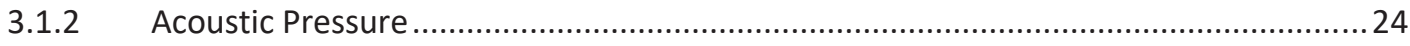

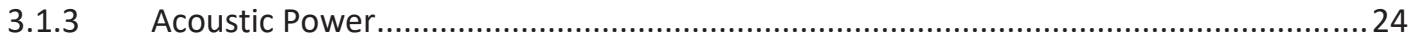

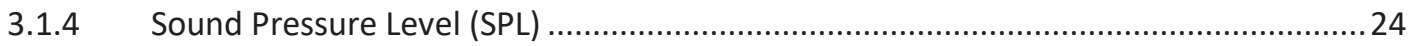

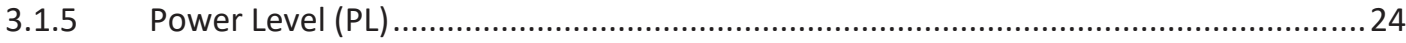

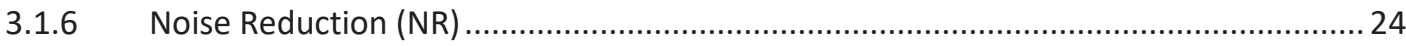

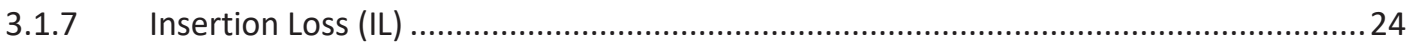

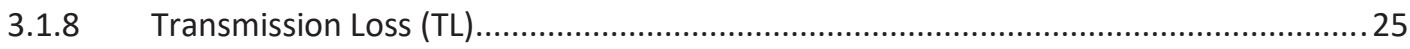

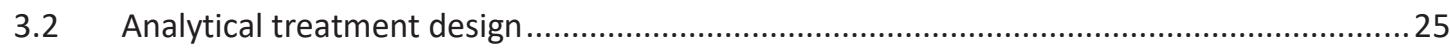

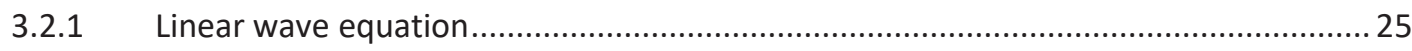

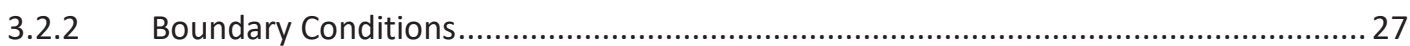




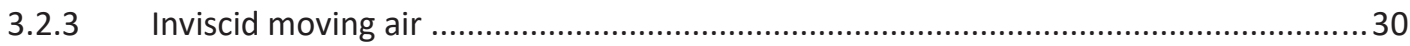

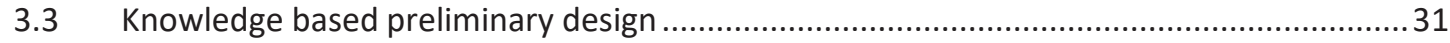

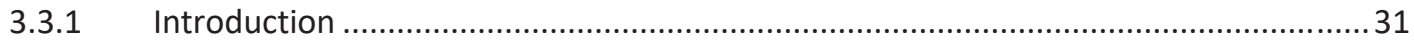

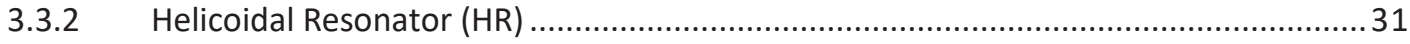

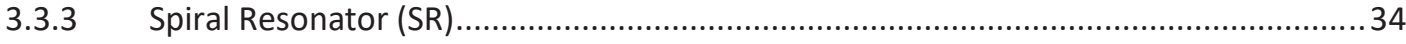

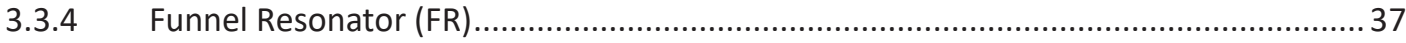

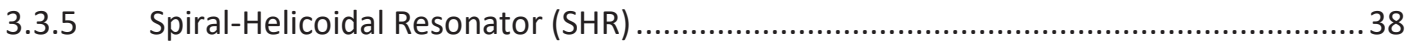

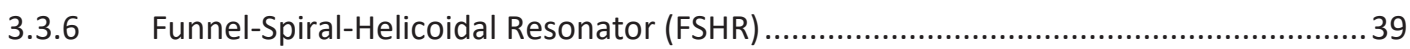

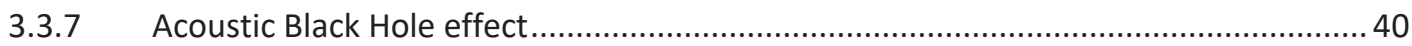

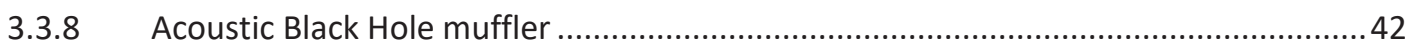

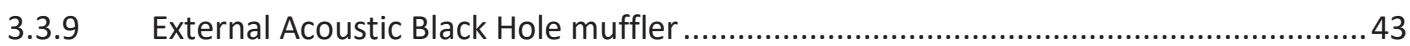

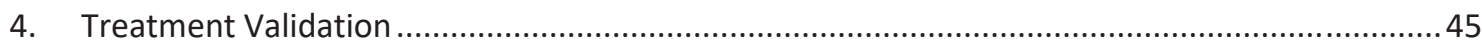

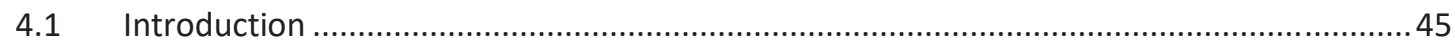

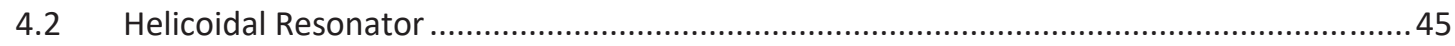

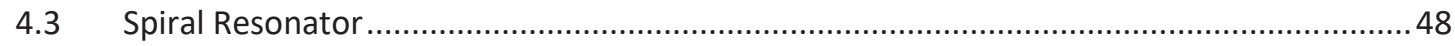

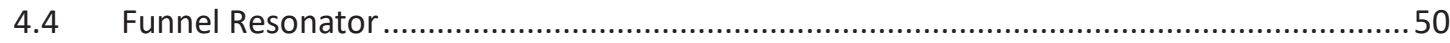

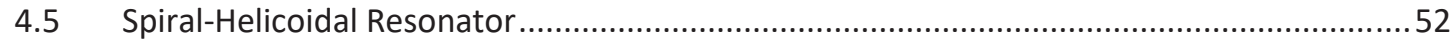

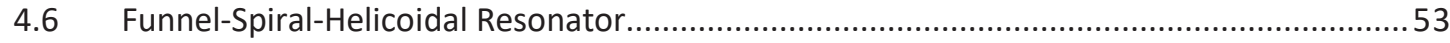

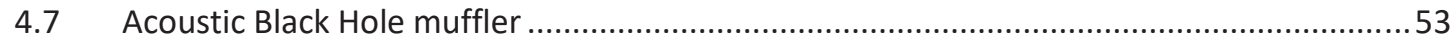

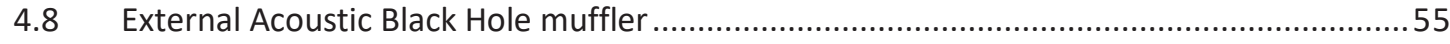

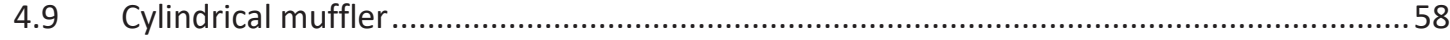

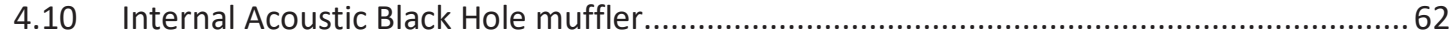

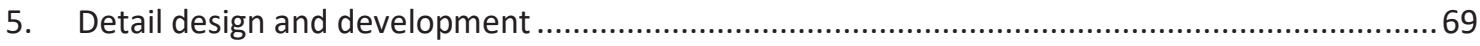

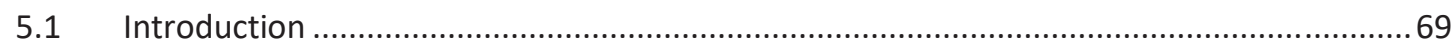

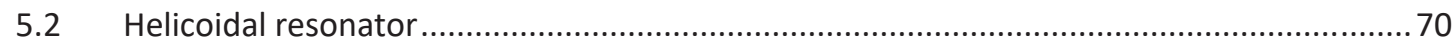

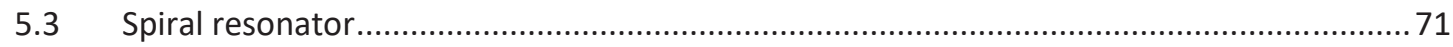

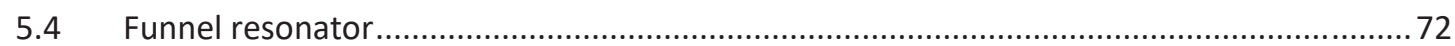

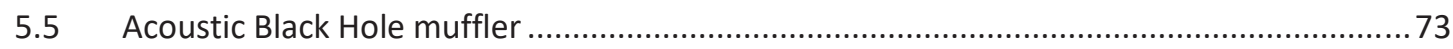

5.6 External Acoustic Black Hole muffler ............................................................................. 74

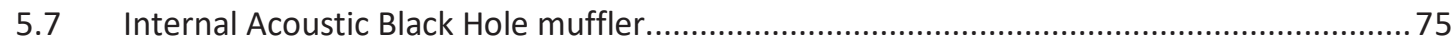

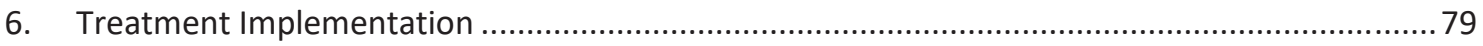

6.1 Test setup

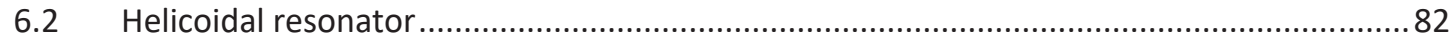

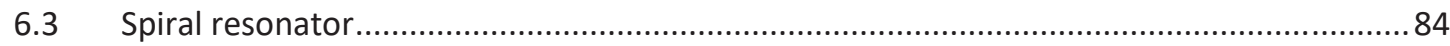

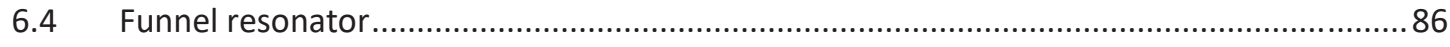

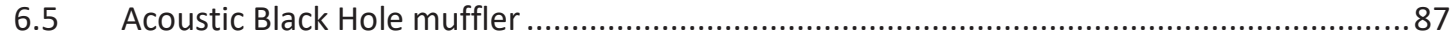




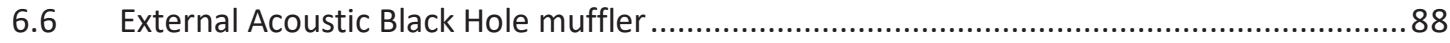

6.7 The combination of the External and internal Acoustic Black Hole resonators ...................91

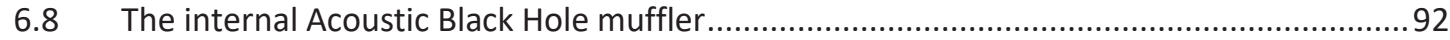

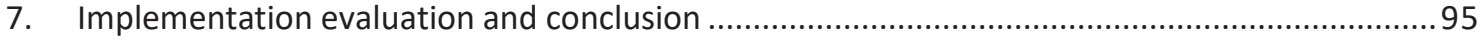

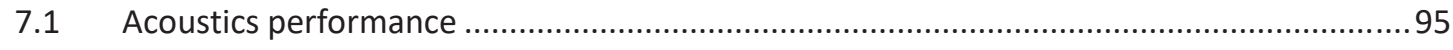

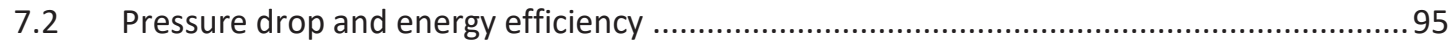

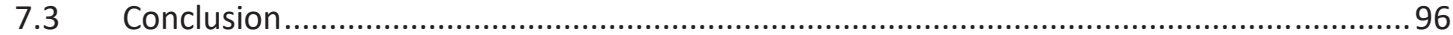

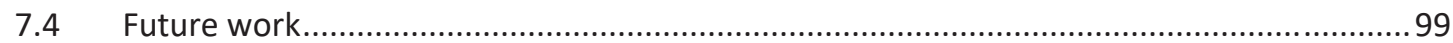

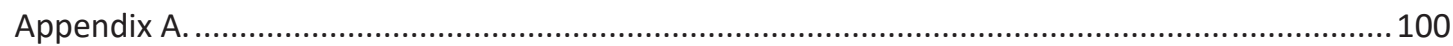

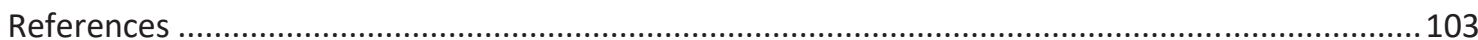

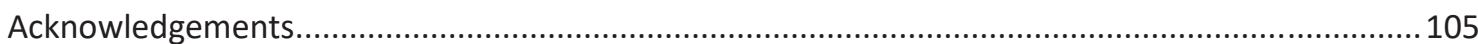





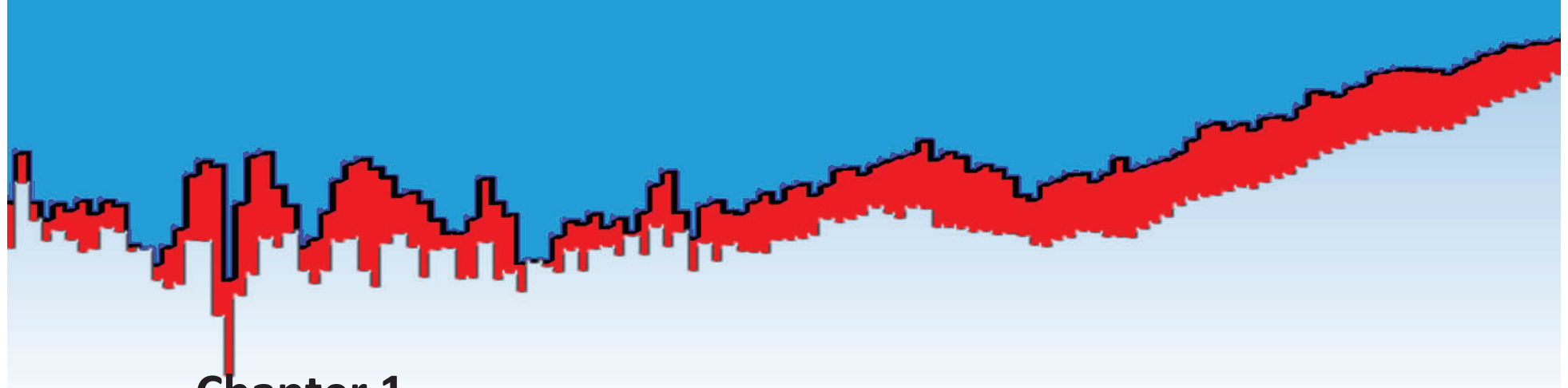

\section{Chapter 1}

\section{Introduction}

\subsection{Problem Statement}

The significance of ventilation systems in today's modern world is evident. In some cold areas, these systems are used without cooling cycles with the main function to send fresh air inside the house. But for a lower energy consumption in winter, heat recovery technologies are applied to preheat the fresh air with exhausted air before being supplied to the residential space. An overview of the ventilation system is presented in Fig. 1.1.

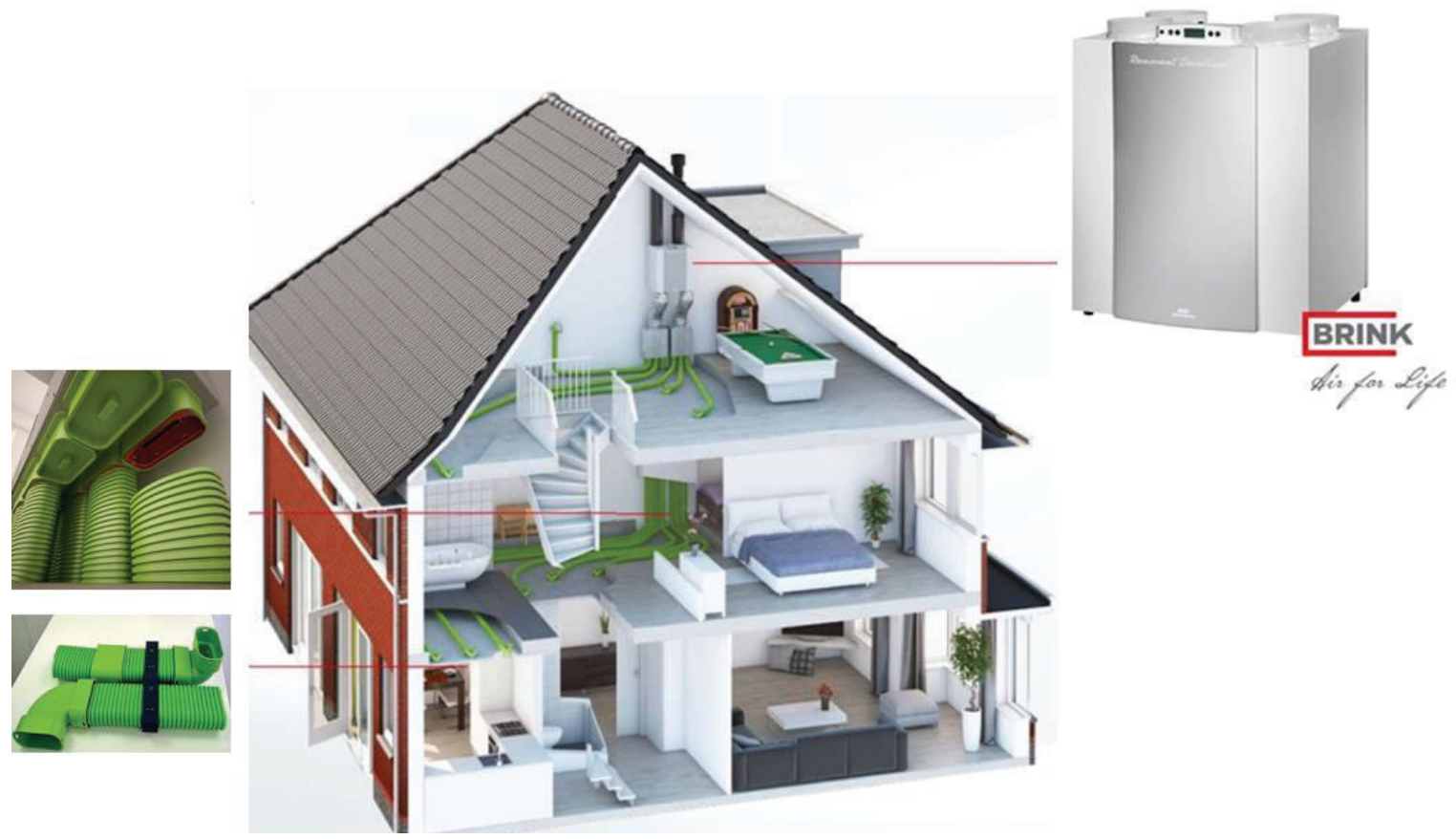

Figure 1.1: General view of a ventilation system in a house.

This ventilation system has two fans to blow the inlet and outlet air and produce sufficient pressure to overcome the pressure drops in inlet and outlet ducts. Besides breathing fresh air, customers have additional priorities. The important one is a low level of produced sound (noise). For low air flowrates, one of the best options is utilizing centrifugal fans. These fans, 
however, are the primary source of the noise in the unit. The noise sources are mechanical vibrations due to the fan as the primary source, the acoustic pressure generated by the action of the impeller in air and the turbulence of the air in the ducts known as aeroacoustics noise. This acoustic pressure can become audible through the air supply vents. The level of the noise is crucial for the acceptance by the users. If the noise level is too high, many users reduce the amount of air flow. In some cases, this will lead to insufficient ventilation with possible consequences regarding health.

\subsection{Objective}

In this project, the goal is to reduce the noise radiated from the air supply vents. The dominant noise level is in the frequency range of $250 \mathrm{~Hz}$ to $2500 \mathrm{~Hz}$. Because the standard acoustic dampers are big when they are to be effective at low frequencies, the objective of this project is to design small appliances which emit significantly lower noise levels. It should be mentioned that these appliances may produce pressure drops inside the duct, so the fan needs to work harder and more input power is consumed to blow the same amount of airflow.

\subsection{Design challenge}

The design challenges can be formulated as follows.

- How can the Sound Power Level of the ventilation system be reduced by $10 \mathrm{~dB}$ ?

- Which type of noise reduction methods can be applied in this project?

- How much an input power of the unit will be increased?

\subsection{Company}

Brink Climate Systems is one of the leading manufacturers of ventilation systems. This company is active on the European market and in some Asian countries. Brink Climate Systems has approximately 180 employees and it is part of CENTROTEC Sustainable AG. CENTROTEC is a listed company with offices in 50 countries and nearly 3300 employees. Brink is one of the brands in the Climate Systems segment. With Brink's solutions in the fields of ventilation, heating, cooling and hot water generation, people live and work healthy, comfortable, and sustainable for a lifetime [1]. 


\subsection{Design approach}

Design science is a design of artifacts to interact with a problem context in order to meet desired goals [2]. The design and investigation process has two parts, i.e., design problems and knowledge questions. A design problem is a problem to (re)design an artifact and a knowledge question asks for the related knowledge [2]. The design quality depends on the quality of the design process. The design process is mostly presented in form of a phase-model which contains some steps that a designer should carry out [3]. Four main steps in this respect can be expressed as 1) an analysis and investigation of the problem, 2) a design of a solution or treatment, 3) an implementation of the treatment, and 4) an evaluation of the treatment. This model is called ADIE-model (Analysis, Design, Implementation, Evaluation) [3]. The more detailed design process can be seen in an engineering cycle. The engineering cycle is a rational process of solving the problem which is shown in Fig. 1.2 [2]. The first three design tasks, i.e., problem investigation, treatment design, and treatment validation are named a design cycle. The design cycle reduces uncertainty about the treatment and designs are validated before implemented. In the next steps of the engineering cycle, first it requires choice of a design results of the design cycle, and commitment to implement the design, then it will be applied in the real environment and evaluated there [2].

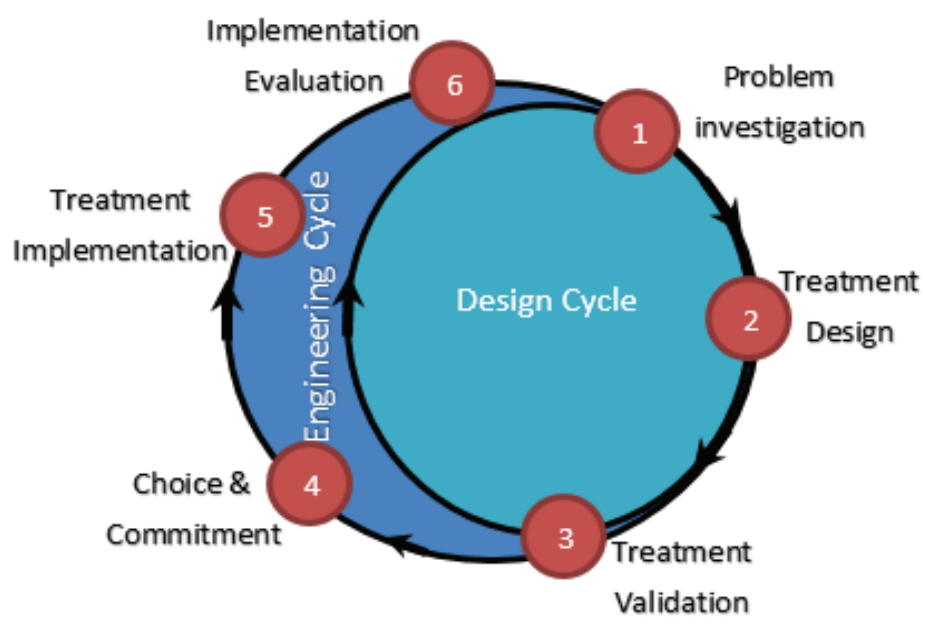

Figure 1.2: Design and Engineering cycles.

\subsection{Outline of the PDEng thesis}

Reducing the low frequency noise in ventilation systems can be considered as a main challenge for the manufacturers of these systems. The objective of this project is designing a 
silencer as an accessory for the ventilation system to supply fresh air with $10 \mathrm{~dB}$ less Sound Power Level (SPL). This goal can be achieved by using various types of noise reduction methods. First of all, the noise can be reduced from the sound sources, i.e., the fans. Secondly, the so-called air-borne radiation, i.e., the aeroacoustics noise, can be absorbed after being emitted from the source. Thirdly, and in contrast with the second category, the so-called structure-borne radiation, i.e., the vibro-acoustic noise, can be absorbed from the structure of the unit itself. After a definition of the various noise reduction methods [4], the priority of each method is estimated using a Value Engineering procedure [5]. Based on the priorities, the best methods are selected. Next, upon finding and selecting the methods, the methods will be validated by design and simulation with SolidWorks, COMSOL, and MATLAB software. Furthermore, the detail design parameters will be defined. In Brink Climate Systems, subsequent development stages of a prototype are indicated with the designations Sample A to Sample D. In this project, Samples A to C will be developed, built, and tested. Next, the results are evaluated and if it is acceptable for the market, then Sample D of the new ventilation unit will be produced.

\subsection{Thesis layout}

In the second chapter of this study, the problem investigation, the stakeholders, and their goals are introduced. Then, the requirements are defined and the design approach is examined.

In the third chapter, after defining acoustic parameters and the ruling equations in this area, the analytical treatments are presented, subsequently knowledge based preliminary design is done.

The fourth chapter deals with the validation of each treatment by using the numerical software, i.e., SolidWorks, COMSOL, and MATLAB.

In the fifth chapter, based on the previous knowledge based preliminary design and the numerical results, the detail design of each treatment is presented.

The sixth chapter concerns preparing the test setup and implementing the treatments in the real environment. Then, this implementation is evaluated in the seventh chapter in which the Technical Performance Measures (TPMs) values are compared with each other.

Finally, the conclusion of the project is given and some suggestions for the future work are discussed. 


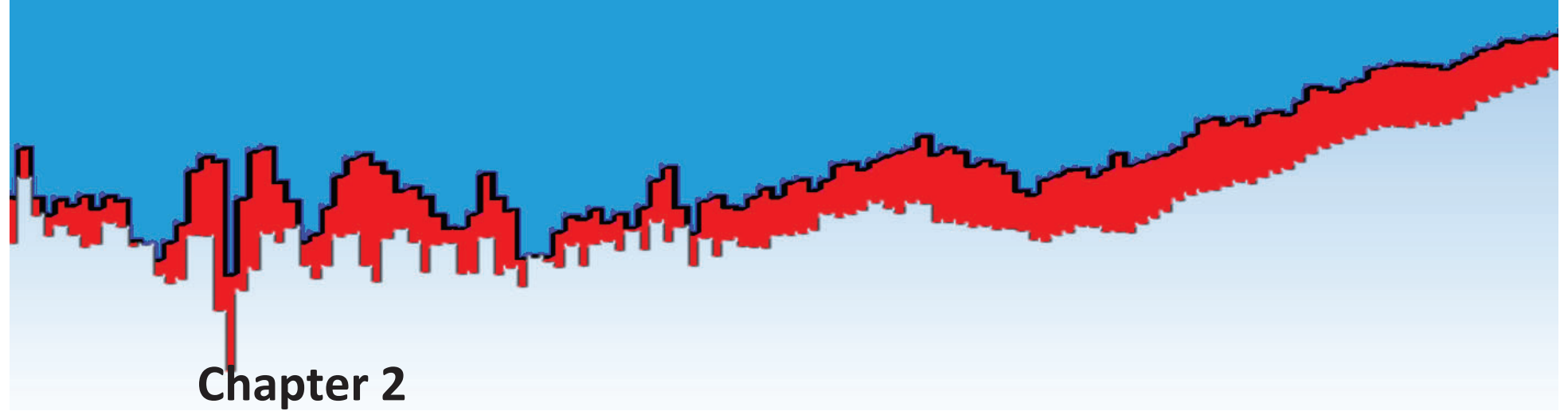

\section{Problem investigation}

\subsection{Introduction}

During the problem investigation step, it is necessary to define stakeholders, their needs, and goals. The next part of this step is determining the design framework, and mechanism. In addition, needs of stakeholders should be transformed into requirements. Then, available treatments might be found that attain the goals.

\subsection{Stakeholders}

A stakeholder is a group of people or institutions affected by the results of the project. Based on Alexander's list [2], the stakeholders in this project are:

- Normal operators: End users.

- Maintenance operators: Service technician of Brink Climate Systems.

- Functional beneficiaries: Company's managers, supervisors at the University of Twente.

- A financial beneficiary: Brink Climate Systems, University of Twente.

- A political beneficiary: Government.

- A negative stakeholder: Companies with the former technologies.

- A threat agent: Competitors of the company.

- The sponsor: Brink Climate Systems and the University directly and the government indirectly.

- The purchaser: R\&D department's manager at Brink Climate Systems.

- Suppliers: Injection molding Companies.

\subsection{Goals}

Goals are desires of stakeholders. Some of the goals of each stakeholder are written as below however, the main goal is reducing the noise level of the ventilation system:

- End users: Less noise, less size of the ventilation unit and supplying enough airflow.

- Service technician: Increasing the maintenance interval and maintainability.

- Company managers: Gain a better market. 
- Supervisors at the university: Solving the problem using noise reduction theories.

- University of Twente: Getting more reputation and more industrial projects.

- Government: Reducing a health problem in the society.

- R\&D manager: Team working, make a patent, developing new technologies.

- Suppliers: Making the model with the Plastic injection molding technique.

\subsection{Requirements}

To define requirements, first it is needed to know more about the ventilation unit and its specification. A Flair 325 is a novel development with high-efficiency that it can supply the correct airflow rate in any situation. Fig. 2.1 shows the overall view of various entities of the Flair 325 and the description of the Fig. 1.2 is depicted in Table.2.1 [6].

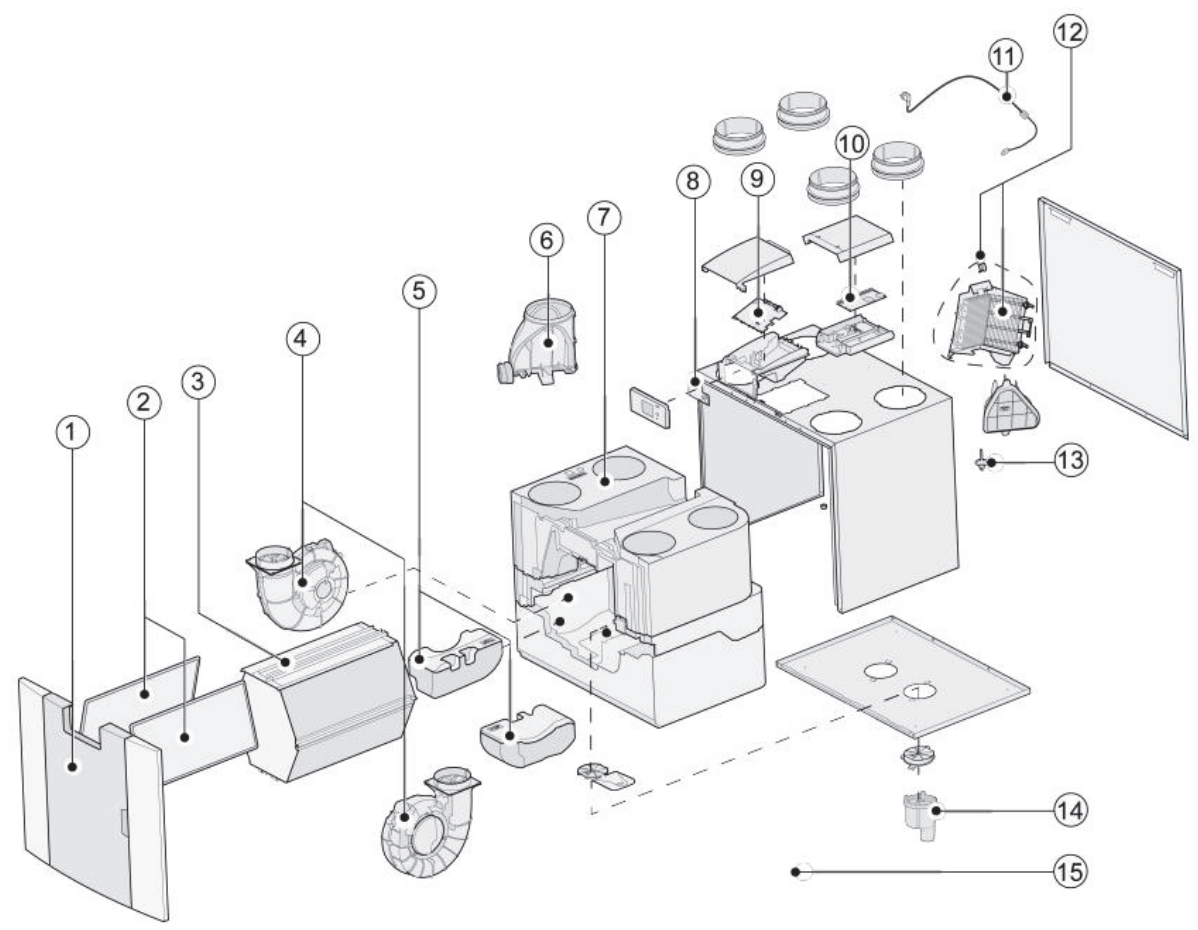

Figure 2.1: Overall view of various entities of the Flair 325 [6].

Table 2.1: Description of the Flair 325.

\begin{tabular}{|l|c|c|c|}
\hline & & Flair 325 & \\
\hline $\mathbf{1}$ & Plastic front panel & 9 & Basic pcb UWA2-B \\
\hline $\mathbf{2}$ & Filters (2 items) ISO Coarse 60\% & 10 & Plus pcb UWA2-E \\
\hline $\mathbf{3}$ & Heat exchanger & 11 & Mains plug and cable 230 V \\
\hline $\mathbf{4}$ & Fan (1 item) & 12 & Internal preheater incl. maximum security \\
\hline $\mathbf{5}$ & EPS assembly & 13 & Temperature sensor \\
\hline $\mathbf{6}$ & Bypass valve with motor & 14 & Condensation discharge \\
\hline $\mathbf{7}$ & EPP interior & 15 & Cable set \\
\hline $\mathbf{8}$ & Display pcb UBP-2 & & \\
\hline
\end{tabular}


Hence, requirements for this project are defined as below:

1. Satisfy ISO 3741 standard.

2. Sound Power Level should be less than $60 \mathrm{~dB}$ at $325 \mathrm{~m}^{3} / \mathrm{h}$ air flowrate and $150 \mathrm{~Pa}$ pressure differences between the inlet and outlet ducts of the unit.

3. External Muffler's size should be less than $1 \mathrm{~m}$ in length and $300 \mathrm{~mm}$ in outside diameter and more than $125 \mathrm{~mm}$ in inside diameter.

4. Size of the unit cannot be extended (dimensions $(H \times W \times D): 650 \times 750 \times 560 \mathrm{~mm}^{3}$ ).

5. Pressure drop of the appliance inside the duct should be less than $50 \mathrm{~Pa}$.

6. Modifications should not have any drawbacks in terms of health issues.

\subsection{Assumptions}

1. Airflow range is 50 to $325 \mathrm{~m}^{3} / \mathrm{h}$.

2. Temperature range is $16{ }^{\circ} \mathrm{C}$ to $35^{\circ} \mathrm{C}$.

3. Humidity range is 30 to $90 \%$.

4. Ducts are smooth.

5. Air flow is turbulent after emitting from the fan.

6. The dust-particle size is negligible.

\subsection{Framework}

The conceptual framework can be used to frame a research problem, describe the phenomena, and analyze the structure. Architectural and statistical structures are two main types of structures. The architectural structure is useful in case-based research, while the statistical structure supports sample-based research. This project is case-based research, and architectural structure is a proper structure to consider.

\subsection{Design Mechanism}

There are many ways to investigate implementation and find the mechanism of the problems like Survey, Observational Case Studies, Single-Case Mechanism Experiments, and Statistical Difference-Making Experiments. The applicable methods in this project could be determined by literature study and talking with product developer R\&D, R\&D manager of the Company, supervisors at the university as experts in this field. So, the first mechanism is expert opinion. Afterwards, it is needed to use some limited models and compare the results, which can be considered as Statistical Difference-Making Experiments mechanism. Generally, in statistical difference-making experiments, an artifact is tested by using it to treat a sample of population elements [2]. Finally, the new appliance will be built and tested by considering Technical 
Action Research mechanism i.e. a newly designed artifact is tested in the field by using it to help a client [2]. This stage will be the last stage in scaling up a technology from the laboratory to the real world which is called Scaling Up Mechanism.

\subsection{Process model}

We have three well-known process models as waterfall, spiral, and Vee models. To select which one is the best for a specific project, it is needed to study the literature and practice. In this project, due to technical tests in each step, the Vee model can be used effectively. Applicable criteria regarding the system should be expressed in terms of technical performance measures (TPMs) and exhibited at the system level. Technical performance measures are quantitative values (estimated, predicted and measured) that describe system performance. Some of the TPMs in this project are Sound Power Level (PL), Sound Pressure Level (SPL), Insertion Loss (IL), Transmission Loss (TL), size, airflow capacity. To ensure that all the requirements are met, the TPMs will be tracked throughout the design process and we use the scheduling formal design review. Also, the TPMs will be checked with all members of the group by sending an email or at face to face steering meetings and so on.

\subsection{Design process algorithm}

One of the reliable methods to get the treatment from requirements is depicted in Fig. 2.2 [7], which is labeled as design process algorithm. Synthesis, analysis, evaluation and modification are tasks that the designer should carry out to get the proper treatment [7].

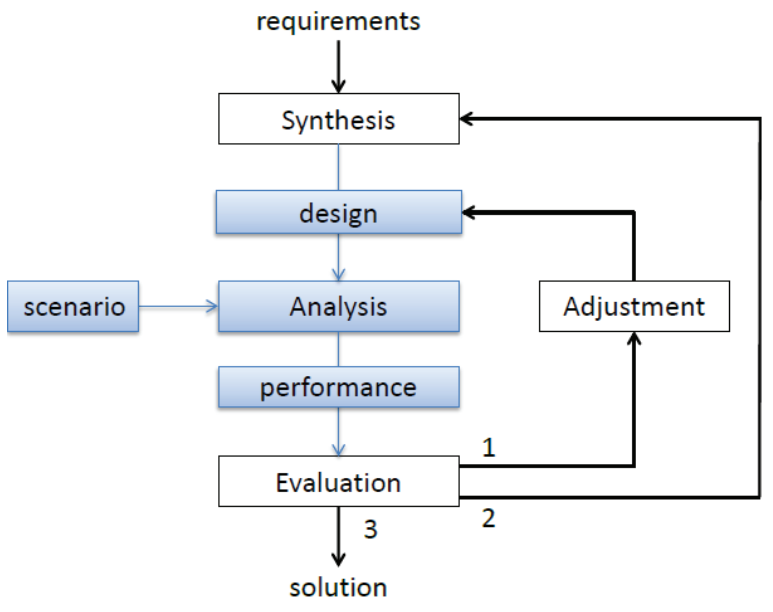

Figure 2.2: Design process algorithm. 
The synthesis task transforms requirements into a possible solution. The performance of the solution can be calculated with the analysis task. Scenario parameters and design parameters play an important role in this step. The parameters which can be changed by the designer are named design parameters and the other parameters like environmental conditions or design limitations which affect the design, but cannot be changed by the designer are called scenario parameters. Then, the performance of the solution is evaluated and the accepted solution might be modified in the adjustment task [8]. The main part of the design process is shown in Fig. 2.1 with blue color, that is proposed Design Process Unit (DPU) [7]. The DPU has four subprocesses as synthesis, analysis, evaluation, and adjustment. Each part has its values, and they can be derived from various methods, but all of them should be applied in design process to obtain goals. Decomposition is one of the main parts of the DPU, and it creates smaller models from a big model. Regarding the decomposition, this project is divided into three sub-projects and each of them has its needs, requirements, designing, implementation, etc. Although these methods are independent from each other, they have interconnection between themselves due to the application in one unit.

\subsection{Function Analysis System Technique}

As mentioned before, the aim of this project is to find good ways to reduce the unit noise as much as possible without decreasing the system's efficiency. Based on a literature review [4], various methods of noise reduction and their functions have been defined. Next, these functions have been categorized by a Function Analysis System Technique (FAST) diagram, as depicted in Fig. 2.3. 


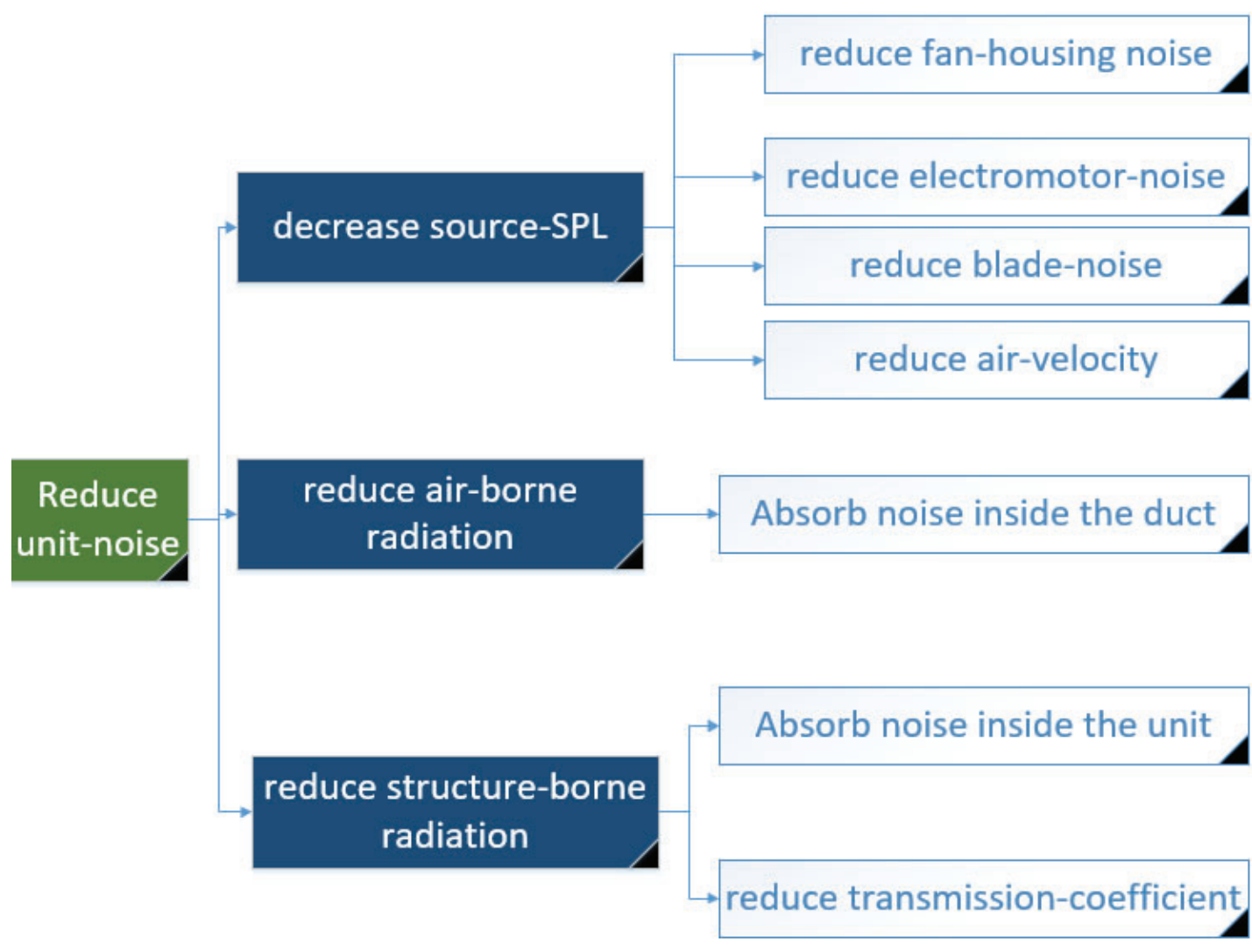

Figure 2.3: FAST diagram of the noise reduction methods.

\subsection{Treatments overview}

According to Fig. 2.3, different functions and solutions are taken into account to reach the final goal. For instance, some modifications can be made in order to have good flow streamlines inside the fan-housing such as adjusting the geometry of the volute tongue, changing the material of the fan-housing, using porous materials covered by thin layers, and last but not least installing a Helmholtz resonator at the volute tongue [4]. The purpose of these modifications is to reduce the noise source sufficiently. To following attributes can contribute in reducing structure-borne noise: the location of the fans, the correct selection of the unit body material, the size of the unit, the sizes of the inlet and outlet ducts, the position of the heat exchanger, the insulation joints, and finally the installment of a vibration absorber. The combination of the previously mentioned attributes can all be used in order to reduce the structure-borne radiation. Finally, by applying the various types of mufflers and resonators, the noise can be reduced in a significant way. In this case, various techniques in active and passive categories can be taken into account to attenuate the duct noise. Many manufacturers prefer to use passive techniques instead of active ones due to the cost and 
necessary control systems. The passive techniques are divided into two parts, termed internal and external methods. Some of the internal passive techniques are the Internal Acoustic Black Hole muffler (IABH), the Helicoidal Resonator (HR), the Spiral Resonator (SR), and the Funnel Resonator (FR); also for the external methods, the hybrid muffler, the External Acoustic Black Hole muffler (EABH), the Cylinder Muffler (CM) could be considered.

Based on Value Engineering, the suggested methods have been evaluated in terms of the criteria listed below; each criterion is characterized by its own weighting factor (WF):

1. Power consumption by adding new entities for noise reduction (WF: 4)

2. Unit noise radiation (WF: 5)

3. Duct noise (WF: 5)

4. Lifetime (WF: 3)

5. Reliability (WF: 4)

6. Maintainability (WF: 3 )

7. Maintenance interval (WF: 2)

8. Adjustment needed (WF: 1)

9. Clean air (WF: 5)

10. Volume (WF: 3)

11. Development time (WF: 2)

12. Development cost (WF: 2)

13. Cost (increasing the total price of the unit by adding a new entity) (WF: 4).

The comparison matrix is illustrated in Table. 2.2 where the values of each method are accordingly listed along with their priorities set by Brink Climate Systems, with $A$ the highest priority and $D$ the lowest priority. 


\begin{tabular}{|c|c|c|c|c|c|c|c|c|c|c|c|c|c|c|c|c|c|c|c|c|c|c|c|}
\hline 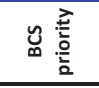 & & 0 & 0 & 0 & $\infty$ & $u$ & 0 & 0 & $\circ$ & $\varangle$ & $\infty$ & $u$ & $<$ & $\varangle$ & $\infty$ & $\infty$ & $\varangle$ & $<$ & $<$ & $\varangle$ & $<$ & $<$ & $\ll$ \\
\hline$\frac{\mathrm{g}}{\frac{3}{3}}$ & & $\approx$ & F & ஃ & $\stackrel{\circ}{\circ}$ & $\stackrel{\infty}{\stackrel{-}{\circ}}$ & ณ & ఏ్ન & 8 & 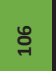 & $m$ & 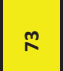 & $\stackrel{\vec{\sigma}}{0}$ & D्ञ & $\stackrel{\infty}{\exists}$ & ๓ & $\stackrel{\circ}{\circ}$ & $\stackrel{乛}{~}$ & $\approx$ & స్తి & : & $\stackrel{\infty}{\stackrel{-}{-1}}$ & g \\
\hline 茄 & $\sigma$ & 요 & 0 & $\infty$ & $\infty$ & $\infty$ & $\infty$ & $\infty$ & $\infty$ & $\infty$ & 0 & $\bullet$ & n & $\infty$ & I) & $\infty$ & $\infty$ & i & $a$ & $\sigma$ & $\infty$ & $\infty$ & $\stackrel{9}{9}$ \\
\hline 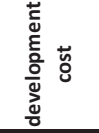 & $\sim$ & $\infty$ & 0 & $\infty$ & $\infty$ & 6 & $\sigma$ & 0 & $\infty$ & $\infty$ & 0 & ${ }^{\circ}$ & $\infty$ & $\circ$ & $\stackrel{9}{9}$ & $\infty$ & ๑ & $\infty$ & 우 & $\stackrel{9}{9}$ & $a$ & $\infty$ & 앙 \\
\hline 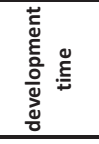 & $N$ & $\infty$ & 0 & $\infty$ & $\infty$ & 0 & $\sigma$ & $\sigma$ & $\infty$ & 0 & $\infty$ & ${ }^{\circ}$ & $\infty$ & $\infty$ & $\stackrel{9}{9}$ & $\infty$ & ๑ & $\infty$ & $\infty$ & $\infty$ & 0 & $\infty$ & $\infty$ \\
\hline 套 & $m$ & o & $\stackrel{\mu}{\circ}$ & $\infty$ & $\infty$ & $\stackrel{9}{9}$ & $\stackrel{9}{9}$ & $\stackrel{r}{\circ}$ & $\sigma$ & $\infty$ & i & $\infty$ & $\infty$ & 6 & $\infty$ & $\infty$ & $\sigma$ & 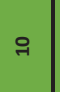 & 이 & ᄋ & $\infty$ & $\stackrel{\sim}{9}$ & $\stackrel{\circ}{-1}$ \\
\hline 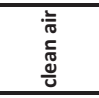 & in & 요 & I & I & 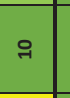 & $\stackrel{7}{9}$ & $\stackrel{\circ}{9}$ & $\stackrel{r}{\circ}$ & 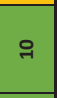 & $\stackrel{\Gamma}{\rho}$ & 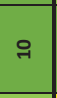 & i & 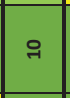 & $\stackrel{9}{9}$ & $\stackrel{9}{9}$ & $\stackrel{\circ}{9}$ & $\infty$ & 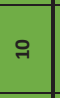 & I & $\stackrel{9}{9}$ & : & 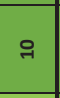 & $\stackrel{9}{9}$ \\
\hline 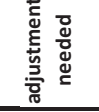 & -7 & o & \& & : & 0 & 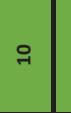 & $\stackrel{9}{9}$ & 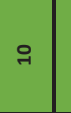 & 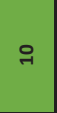 & 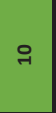 & $\infty$ & 0 & $\stackrel{9}{-1}$ & 웅 & @ & $\stackrel{9}{9}$ & \& & \& & 아 & ᄋ & ๑ & $\infty$ & $\stackrel{\circ}{\circ}$ \\
\hline 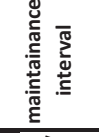 & $\sim$ & $\stackrel{\circ}{\circ}$ & \% & : & $\infty$ & $\stackrel{9}{9}$ & $\stackrel{\circ}{9}$ & I & $\stackrel{\pi}{\circ}$ & $\stackrel{\Gamma}{\circ}$ & $\infty$ & $\circ$ & $\stackrel{\circ}{-1}$ & $\stackrel{\circ}{9}$ & 궁 & $\stackrel{\circ}{9}$ & \& & i & i & $\stackrel{-}{\circ}$ & $\infty$ & $\infty$ & 욱 \\
\hline 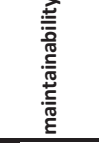 & $m$ & $\stackrel{\circ}{9}$ & \& & $\infty$ & 0 & i & 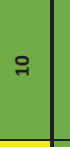 & \% & ๑ & 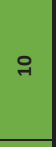 & $\infty$ & $\infty$ & $\stackrel{9}{9}$ & $\infty$ & $\stackrel{\circ}{-1}$ & $\stackrel{\circ}{9}$ & $\infty$ & 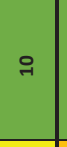 & I & $\stackrel{\circ}{-1}$ & 0 & $\infty$ & 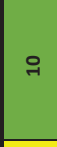 \\
\hline 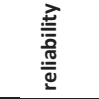 & $\sigma$ & $\sigma$ & \& & 0 & $\infty$ & $\infty$ & 0 & $\infty$ & $\sigma$ & 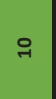 & 0 & $\infty$ & $\stackrel{9}{9}$ & $\infty$ & $\sigma$ & $\infty$ & \& & 0 & $\sigma$ & $\circ$ & 0 & $\infty$ & 0 \\
\hline 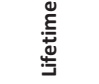 & $m$ & $\infty$ & $\infty$ & $\infty$ & $\infty$ & $\stackrel{9}{9}$ & $\infty$ & ᄋ & $\circ$ & $\infty$ & $\infty$ & $\circ$ & $\infty$ & $\infty$ & 욤 & 0 & \& & g & i & $\infty$ & 0 & $\infty$ & $\stackrel{\circ}{9}$ \\
\hline 芫 & in & $\infty$ & $\infty$ & 0 & o & $\circ$ & $\circ$ & $\infty$ & $\circ$ & ㅇ & $\infty$ & 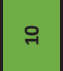 & g & $\infty$ & 。 & $\infty$ & 앙 & $\sigma$ & $\sigma$ & 。 & 용 & $\infty$ & $\circ$ \\
\hline 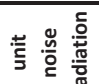 & in & $\circ$ & $\circ$ & ๑ & $\infty$ & $\infty$ & 0 & $\infty$ & $\sigma$ & $\circ$ & $\circ$ & $\infty$ & $\infty$ & 0 & $\sigma$ & $\sigma$ & $\infty$ & $\circ$ & $\circ$ & $\infty$ & $\infty$ & $\infty$ & 요 \\
\hline 竞 & $\sigma$ & 0 & 0 & 요 & q & $\stackrel{\pi}{9}$ & $\infty$ & $\infty$ & $\infty$ & 0 & $\infty$ & $\bullet$ & $\stackrel{9}{9}$ & $\stackrel{9}{9}$ & • & $\sigma$ & 0 & 요 & 우 & $\stackrel{9}{9}$ & $\infty$ & 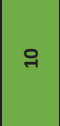 & $\stackrel{\circ}{\longrightarrow}$ \\
\hline 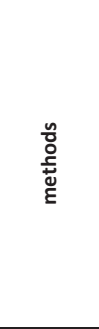 & 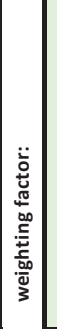 & 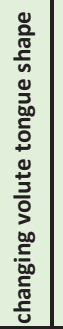 & 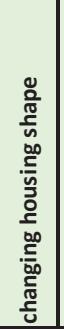 & 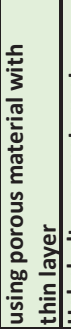 & 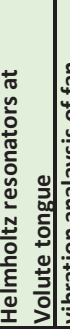 & 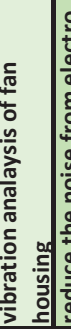 & 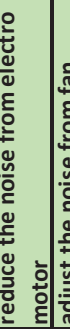 & 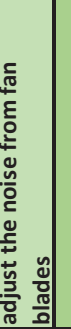 & 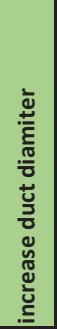 & 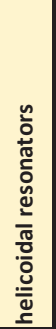 & 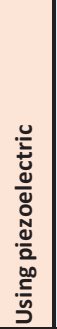 & 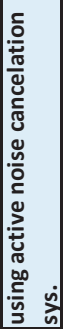 & 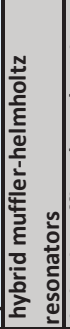 & 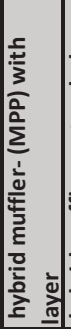 & 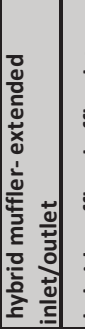 & 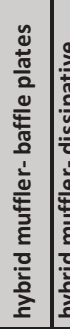 & 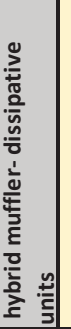 & 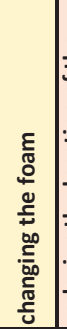 & 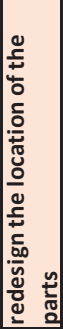 & 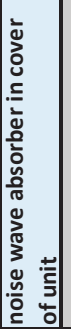 & 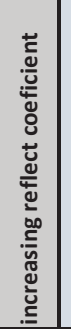 & 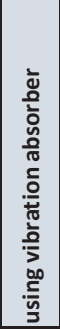 & 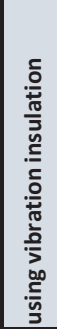 \\
\hline 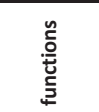 & \multicolumn{9}{|c|}{ 总 } & \multicolumn{8}{|c|}{ 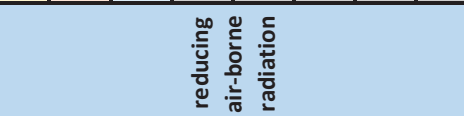 } & \multicolumn{6}{|c|}{ 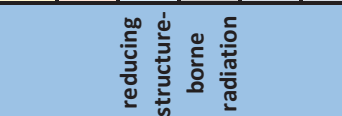 } \\
\hline
\end{tabular}


Based on Table 2.2, some of the methods with larger values and highest priority (A) from the second category have been selected to design and develop the internal and external mufflers and resonators. This project has been divided into three sub-projects.

In the first sub-project, the internal resonators (inside the tube) are designed. The narrowband noise attenuation can be obtained by a properly designed, smart innovation, termed helicoidal resonator, which works as a passive acoustic filter inside the duct. Łapka [9] has simulated the HR inside the common ventilation duct size, $125 \mathrm{~mm}$, and the lowest eigenfrequency that he achieved was $1282 \mathrm{~Hz}$. Finding an analytical solution of the system would be a good way to gain a better understanding of the problem and seek the suitable treatment inside the duct afterwards. The analytical solution helps to develop the other innovative resonators, termed spiral resonator and funnel resonator, which work for frequencies lower than $1282 \mathrm{~Hz}$ which is not possible to achieve by the helicoidal resonator.

In the second sub-project, an Acoustic Black Hole muffler, which absorbs high amount of the incident wave energy, is considered as a passive treatment for low frequency noise reduction inside the duct while the External Acoustic Black Hole (EABH) is considered outside the duct. This absorption takes place by decreasing the velocity of wave propagation towards zero in finite length of the muffler. These mufflers will be installed after the fan to absorb the air noise.

In the third sub-project, in order to apply the treatment in the real environment, the selected method is designed such that it is possible to make it with the injection molding technique and install it inside the ventilation unit. 


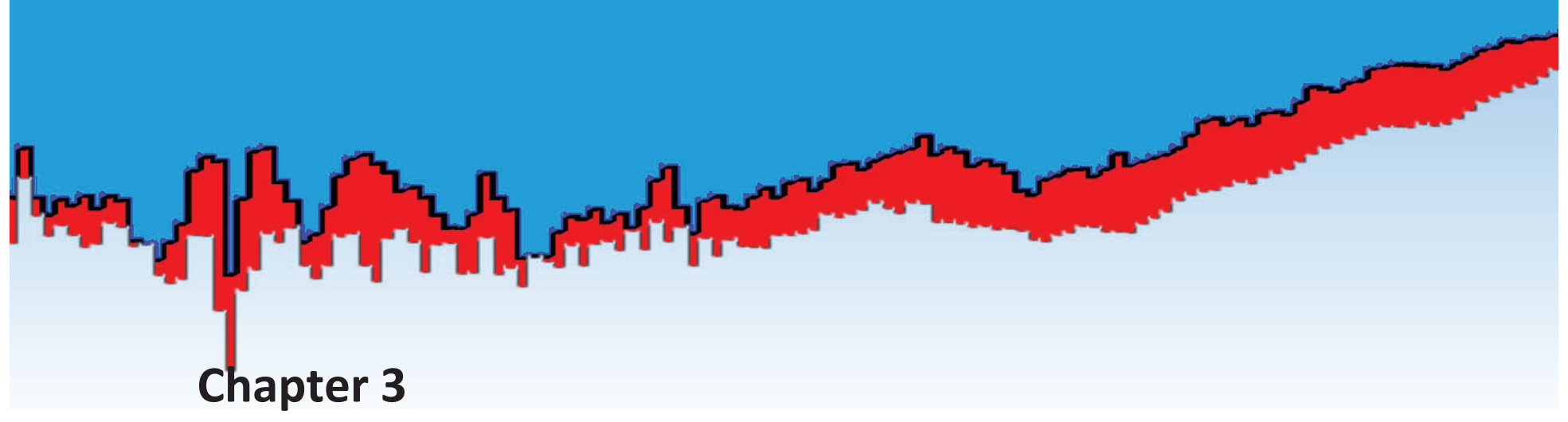

\section{Treatment design}

Treatment design is a design of one or more artifacts that can treat the problem. The goal of treatment design is that some stakeholders are better off when the problem is treated. Treatment design consisted of a literature survey of the state of the art of requirements engineering, followed by the specification of a candidate solution [2]. In this chapter, first some acoustical terms are defined and then the possible analytical treatment will be determined. Based on the analytical treatment, some resonators are designed in the knowledge based preliminary design phase.

\subsection{Definitions}

\subsubsection{Acoustics}

The word acoustics is derived from the Greek word root Akuein meaning hearing. It was first used by Sauveur for this science. Acoustics as a science began with Pythagoras, who wanted to know why some musical sounds are more interesting than others which he found out in the numerical proportions. Aristoteles understood that sound is composed of air expansion and contraction. Galileo and Mersenne independently discovered and completed the law of vibrating strings that Pythagoras had started 2000 years ago [10]. Acoustics as a science may be defined as the generation, transmission, and reception of energy as vibrational waves in matter [11]. 


\subsubsection{Acoustic Pressure}

The difference between instantaneous pressure $P$ and static (equilibrium) pressure $p_{0}$ is termed sound pressure or acoustic pressure $p[12]$ :

$$
p=P-p_{0} .
$$

\subsubsection{Acoustic Power}

Acoustic power or sound power is the product of the sound pressure $p$ and the component of the particle velocity, $u_{n}$, at a point on a surface in the direction normal to the surface, integrated over that surface [12] [13]:

$$
w=\int p u_{n} d A
$$

\subsubsection{Sound Pressure Level (SPL)}

Sound Pressure Level is ten times the logarithm to the base 10 of the ratio of the square of the sound pressure $p$ to the square of a reference value $p_{0}=20 \mu \mathrm{Pa}$ expressed in decibels (dB) [12]:

$$
S P L=10 \log \left(\frac{p^{2}}{p_{0}^{2}}\right)=20 \log \left(\frac{p}{p_{0}}\right) .
$$

\subsubsection{Power Level (PL)}

Power Level is ten times the logarithm to the base 10 of the ratio of the sound power of a source $w$ to a reference value $w_{0}=10^{-12} \mathrm{~W}$ expressed in decibels [12]:

$$
P L=10 \log \left(\frac{w}{w_{0}}\right)
$$

To define the efficiency and performance of the noise reduction systems, various types of methods can be used. The most important ones are expressed below.

\subsubsection{Noise Reduction (NR)}

The Noise Reduction (NR) parameter is the difference between the sound pressure levels at two arbitrary selected points like inlet and outlet of the duct [13]:

$$
N R=S P L_{\text {in }}-S P L_{\text {out }} .
$$

\subsubsection{Insertion Loss (IL)}

Insertion Loss difference between acoustic power level without any filter and with filter [13]:

$$
I L=\text { Power Level Out } t_{\text {withoutFilter }}-\text { Power LevelOut }{ }_{\text {withFilter }} .
$$




\subsubsection{Transmission Loss (TL)}

The Transmission Loss (TL) describes the performance of the model by finding the differences between Sound Power Level before and after the model inside the tube [13]:

$$
T L=\text { Power Levl } l_{\text {in }}-\text { Power Level } l_{\text {out }}=10 \log \left(\frac{\text { Acoustic power }_{\text {in }}}{\text { Acoustic power }_{\text {out }}}\right) .
$$

\subsection{Analytical treatment design}

\subsubsection{Linear wave equation}

When the fluid element with velocity $v(x, y, z, t)$ at position $(x, y, z)$ and time $t$ moves to a new location $(x+d x, y+d y, z+d z)$ at a later time $t+d t$, its new velocity is expressed by the leading terms of its Taylor expansion:

$$
v=v\left(v_{x}, v_{y}, v_{z}, t\right)+\frac{\partial v}{\partial x} v_{x} d t+\frac{\partial v}{\partial y} v_{y} d t+\frac{\partial v}{\partial z} v_{z} d t+\frac{\partial v}{\partial t} d t
$$

Thus, the acceleration of the chosen element is:

$$
a=\frac{\partial v}{\partial t}+(v \cdot \nabla) v
$$

where $(v . \nabla)=v_{x} \frac{\partial}{\partial x}+v_{y} \frac{\partial}{\partial y}+v_{z} \frac{\partial}{\partial z}$. By considering $\quad d m=\rho d V, \quad d f=a d m$ and $d f=-\nabla p d V+g \rho d V$ it can be shown [11]:

$$
-\nabla P+g \rho=\rho\left(\frac{\partial v}{\partial t}+(v \cdot \nabla) v\right)
$$

This nonlinear, inviscid force equation is Euler's equation with gravity. In the case of no acoustic excitation $\nabla P_{0}=g \rho_{0}$ and thus $\nabla P=\nabla p+g \rho_{0}$ so it becomes:

$$
-\frac{1}{\rho_{0}} \nabla p+g s=(1+s)\left(\frac{\partial v}{\partial t}+(v . \nabla) v\right), s=\frac{\rho-\rho_{0}}{\rho_{0}} .
$$

If we now make the assumptions that $|g s|<<|\nabla p| / \rho_{0},|s|<<1,|(v . \nabla) v|<<\left|\frac{\partial v}{\partial t}\right|$ and take the divergence of the above equation, then:

$$
\nabla \cdot\left(\rho_{0} \frac{\partial v}{\partial t}\right)=-\nabla^{2} p
$$


where $\nabla . \nabla=\nabla^{2}$ is the three-dimensional Laplace operator. Next, take the time derivative of the continuity equation and use the facts that space and time are independent and that there are no large density variations over time:

$$
\rho_{0} \frac{\partial^{2} s}{\partial t^{2}}+\nabla \cdot\left(\rho_{0} \frac{\partial v}{\partial t}\right)=0
$$

By using these two equations, we obtain the linear lossless wave equation as [11]:

$$
\nabla^{2} p-\frac{1}{c_{0}^{2}} \frac{\partial^{2} p}{\partial t^{2}}=0,
$$

where $c_{0}$ is the thermodynamic speed of sound defined as:

$$
c_{0}{ }^{2}=\left.\frac{\partial P}{\partial \rho}\right|_{\text {adiabat }}=\frac{\beta}{\rho_{0}} .
$$

Hence, in cylindrical-coordinates an acoustic pressure $p$ at each point $(r, \varphi, z, t)$ inside the duct could be derived by solving wave equation below:

$$
\nabla^{2} p(r, \phi, z, t)-\frac{1}{c_{0}^{2}} \frac{\partial^{2} p(r, \phi, z, t)}{\partial t^{2}}=0
$$

where $\nabla^{2} \equiv \frac{\partial^{2}}{\partial r^{2}}+\frac{1}{r} \frac{\partial}{\partial r}+\frac{1}{r^{2}} \frac{\partial^{2}}{\partial \varphi^{2}}+\frac{\partial^{2}}{\partial z^{2}}$. Using the separation of variables method, the general solution for wave equation could be expressed as [14]:

$$
p(r, \phi, z, t)=R(r) \times \Phi(\phi) \times Z(z) \times T(t) .
$$

The expression for each term is written as [13],[14],[15]:

$$
\begin{aligned}
& \left\{\begin{array} { c } 
{ \frac { d ^ { 2 } T } { d t ^ { 2 } } + \omega ^ { 2 } T = 0 , \omega = 2 \pi f = c _ { 0 } k } \\
{ T ( t ) = e ^ { i \omega t } }
\end{array} \left\{\begin{array}{c}
\frac{d^{2} Z}{d z^{2}}+k_{z}{ }^{2} Z=0 \\
Z(z)=A_{1 z} e^{-i k_{z} z}+A_{2 z} e^{i k_{z} z}
\end{array}\right.\right. \\
& \left\{\begin{array} { c } 
{ \frac { d ^ { 2 } \Phi } { d \phi ^ { 2 } } + m ^ { 2 } \Phi = 0 } \\
{ \Phi ( \phi ) = A _ { 1 \phi } \operatorname { c o s } ( m \phi ) + A _ { 2 \phi } \operatorname { s i n } ( m \phi ) }
\end{array} \quad \left\{\begin{array}{c}
r^{2}\left(\frac{\partial^{2} R}{\partial r^{2}}+\frac{1}{r} \frac{\partial R}{\partial r}\right)+\left[k_{r}{ }^{2} r^{2}-m^{2}\right] R=0 \\
R(r)=\sum_{m} A_{m} J_{m}\left(k_{r} r\right)+B_{m} Y_{m}\left(k_{r} r\right)
\end{array}\right.\right.
\end{aligned}
$$

where $\omega$ is the angular frequency, $m$ is a separation constant, and the axial wave number $k_{z}$ can be obtained from the wave number $k_{0}=\frac{\omega}{c_{0}}$ and the radial wave number $k_{r}$ as $k_{z}=\left(k_{0}^{2}-k_{r}^{2}\right)^{0.5}$ for $\omega \geq 0[13]$. 


\subsubsection{Boundary Conditions}

Three different types of boundary conditions are considered. At all the solid boundaries, sound hard (wall) boundary conditions are used [16]:

$$
\left(-\frac{\nabla p}{\rho}\right) \cdot \hat{n}=0
$$

where $\hat{n}$ is a normal vector of the wall's surface. At the inlet boundary for the numerical solution, a combination of an incoming and an outgoing plane wave is assumed. This is explained in the Acoustics module User's Guide of the COMSOL Multiphysics 5.3 software [16], which is validated earlier in these papers [17], [18]:

$$
\left(-\frac{\nabla p}{\rho}\right) \hat{n}=\frac{i \omega}{\rho c_{0}} p-\frac{2 i \omega}{\rho c_{0}} p_{0}
$$

In this equation, $p_{0}$ denotes the applied outer pressure, i.e., the emitted noise from the fan. At the outlet boundary, an outgoing plane wave radiation is set [16]:

$$
\left(-\frac{\nabla p}{\rho}\right) \cdot \hat{n}=\frac{i \omega}{\rho c_{0}} p
$$

For the analytical solution, six boundary Conditions should be defined so that unknown parameters can be found. 
Boundary conditions of the duct with the Open-Open Ends, i.e., Neumann BC's are expressed as below [14]:

$$
\left\{\begin{array} { l } 
{ \frac { d Z ( z = 0 ) } { d z } = 0 } \\
{ \frac { d Z ( z = L ) } { d z } = 0 }
\end{array} \Rightarrow \left\{\begin{array}{c}
Z(z)=\cos \left(k_{z} z\right) \\
k_{z}=2 n \pi / L, n=1,2,3, \ldots
\end{array} .\right.\right.
$$

The axial boundary condition is shown in Fig. 3.1.

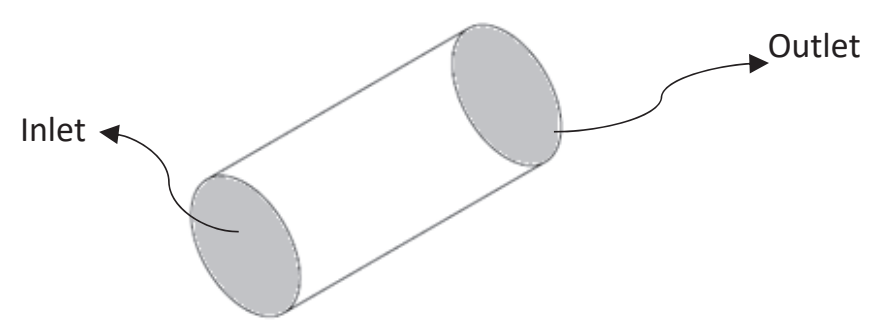

Figure 3.1: Axial boundaries at the simple duct.

\section{Angular Boundary Conditions}

For simple duct in Fig. 3.1, the angular boundary condition should be $\Phi(0)=\Phi(2 \pi)$. In this state, $m$ could be obtained as [15]:

$$
\left\{\begin{array} { c } 
{ \Phi ( 0 ) = A _ { 1 \varphi } } \\
{ \Phi ( 2 \pi ) = A _ { 1 \varphi } \operatorname { c o s } ( 2 m \pi ) + A _ { 2 \varphi } \operatorname { s i n } ( 2 m \pi ) }
\end{array} \Rightarrow \left\{\begin{array}{l}
\sin (2 m \pi)=0 \\
\cos (2 m \pi)=1
\end{array} \Rightarrow m=0, \pm 1, \pm 2, \pm 3, \ldots .\right.\right.
$$

If the duct includes a blade like in Fig. 3.2 then $m$ becomes $m=0, \pm 0.5, \pm 1, \pm 1.5, \pm 2, \ldots$ due to the following conditions [15]:

$$
\begin{gathered}
\frac{d \Phi}{d \phi}(0)=0 \Rightarrow A_{2 \phi}=0 \\
\pi)=0 \Rightarrow-A_{1 \phi} * m * \sin (2 \pi m)=0
\end{gathered} \Rightarrow \begin{gathered}
\Phi(\phi)=A_{1 \phi} \cos (m \phi) \\
m=0, \pm 0.5, \pm 1, \pm 1.5, \pm 2,, \ldots
\end{gathered}
$$

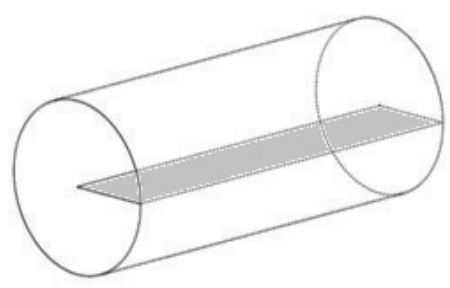

Figure 3.2: Angular boundaries at the duct.

It should be noted that the modes corresponding to $m=0$ are axisymmetric. 
Radial Boundary Conditions

If the duct includes no mandrel $\left(r_{1}=0\right)$ shown in Fig. 3.3 , the pressure at the center of the duct should be limited. Therefore, the coefficient of the second kind of the Bessel Function should be zero and the unknown parameter can be found as [15]:

$$
\left\{\begin{array}{l}
R_{m}\left(r_{1}=0\right): \text { finite } \Rightarrow B_{m}=0 \\
\frac{d R_{m}}{d r}\left(r_{2}\right)=0 \Rightarrow J_{m}^{\prime}\left(k_{r} r_{2}\right)=0
\end{array} .\right.
$$

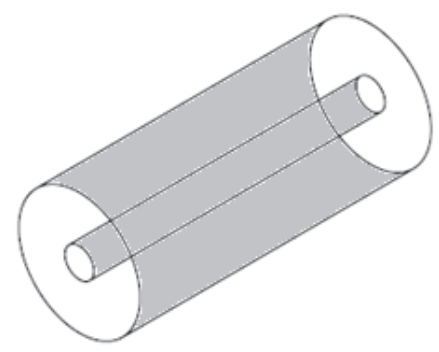

Figure 3.3: Radial boundaries at the duct.

Hence, the radial wave number $k_{r, m}$ will be obtained from the roots of the derivation of the first kind of the Bessel Function equation. The results for various $m$ are written in Table 3.1 [15].

Table 3.1: Roots of the first kind of the Bessel Function.

\begin{tabular}{|l|l|l|l|l|}
\hline Roots of the first kind of the Bessel Function $\left(k_{r, m} \times r_{2}\right)$ & $m=0$ & $m=0.25$ & $m=0.5$ & $m=1$ \\
\hline First root & 0 & 0.7688 & 1.1650 & 1.8410 \\
\hline Second root & 3.8310 & 4.2248 & 4.6040 & 5.3310 \\
\hline Third root & 7.0150 & 7.4062 & 7.7890 & 8.5360 \\
\hline
\end{tabular}

If the duct includes a mandrel or it is a co-axial cylindrical duct, then the pressure derivation at both radial boundaries should vanish [15]:

$$
\left\{\begin{array}{l}
\frac{d R_{m}}{d r}\left(r_{1}\right)=0 \\
\frac{d R_{m}}{d r}\left(r_{2}\right)=0
\end{array} \Rightarrow \frac{d R_{m}}{d r}(r)=B_{m}\left[\frac{A_{m}}{B_{m}} J_{m}^{\prime}\left(k_{r} r\right)+Y_{m}^{\prime}\left(k_{r} r\right)\right]\right.
$$

In this case, $k_{r}$ should be obtained from the following [15]:

$$
\begin{aligned}
& \begin{array}{l}
\frac{A_{m}}{B_{m}} J_{m}^{\prime}\left(k_{r} r_{1}\right)+Y_{m}^{\prime}\left(k_{r} r_{1}\right)=0 \quad \frac{A_{m}}{B_{m}}=-\frac{Y_{m}^{\prime}\left(k_{r} r_{1}\right)}{J_{m}^{\prime}\left(k_{r} r_{1}\right)} \\
\frac{A_{m}}{B_{m}} J_{m}^{\prime}\left(k_{r} r_{2}\right)+Y_{m}^{\prime}\left(k_{r} r_{2}\right)=0
\end{array} \quad \begin{array}{l}
\frac{A_{m}}{B_{m}}=-\frac{Y_{m}^{\prime}\left(k_{r} r_{2}\right)}{J_{m}^{\prime}\left(k_{r} r_{2}\right)} \\
k_{r} r_{2}=X, \frac{r_{2}}{r_{1}}=q
\end{array} \\
& \Rightarrow \frac{A_{m}}{B_{m}}=-\frac{Y_{m}^{\prime}(X / q)}{J_{m}^{\prime}(X / q)}=-\frac{Y_{m}^{\prime}(X)}{J_{m}^{\prime}(X)} \Rightarrow J_{m}^{\prime}(X) * Y_{m}^{\prime}(X / q)-J_{m}^{\prime}(X / q) * Y_{m}^{\prime}(X)=0
\end{aligned}
$$


Values of $X$ for various $m$ and $q$ are given in Table 3.2.

Table 3.2: First and second roots of Eq. (3.26).

\begin{tabular}{|l|c|c|c|c|}
\hline First and second Roots $\left(k_{r, m, q} \times r_{2}\right)$ & \multicolumn{2}{c|}{$m=0$} & \multicolumn{2}{c|}{$m=1$} \\
\hline $\mathbf{q = 2 . 0 8 3 3}$ & First root & second root & First root & second root \\
\hline $\mathbf{q = 4 . 1 6 6 7}$ & 0 & 6.1531 & 1.3744 & 5.7297 \\
\hline $\mathbf{q = 6 . 2 5}$ & 0 & 4.3975 & 1.6563 & 4.9844 \\
\hline $\mathbf{q = 1 2 . 5}$ & 0 & 4.0938 & 1.7494 & 4.9894 \\
\hline $\mathbf{q = 3 1 . 2 5 0 0}$ & 0 & 3.8994 & 1.8163 & 5.1938 \\
\hline
\end{tabular}

By comparing the last row of Table 3.2 with the first and second rows of Table 3.1, it can be concluded that when the mandrel diameter approaches zero, the result of the wave number will approach the results without mandrel. This result also shows that by adding a mandrel inside the duct, lower eigen-frequencies will be obtained.

The pressure function becomes:

$$
P(r, \phi, z, t)==\sum_{m=0}^{\infty} \sum_{n=0}^{\infty}\left[A_{m} J_{m}\left(k_{r, m, n} r\right)+B_{m} Y_{m}\left(k_{r, m, n} r\right)\right]\left[A_{1 \phi} e^{-i m \phi}+A_{2 \phi} e^{i m \phi}\right]\left[A_{1 z} e^{-i k_{z, m, n} z}+A_{2 z} e^{i k_{z, m, n} z}\right] e^{i \omega t}
$$

where

$$
k_{z, m, n}=\left(k_{0}^{2}-k_{r, m, n}{ }^{2}\right)^{0.5} \text { for } \omega \geq 0,
$$

and $n$ is the index of the root of the Bessel function.

\subsubsection{Inviscid moving air}

If an inviscid moving air inside the duct is considered then the general solution will be[13]:

$$
P(r, \phi, z, t)==\sum_{m=0}^{\infty} \sum_{n=0}^{\infty}\left[A_{m} J_{m}\left(k_{r, m, n} r\right)+B_{m} Y_{m}\left(k_{r, m, n} r\right)\right]\left[A_{1 \phi} e^{-i m \phi}+A_{2 \phi} e^{i m \phi}\right]\left[A_{1 z} e^{-i k_{z, m, n}^{+}}+A_{2 z} e^{i k_{z, m, n}^{-}}\right] e^{i \omega t}
$$

where $k_{z, m, n}^{+}$and $k_{z, m, n}^{-}$are governed by:

$$
k_{z, m, n}^{2}+k_{r, m, n}^{2}=\left(k_{0}+M k_{z, m, n}\right)^{2}
$$

Here, the mean-flow velocity is assumed to be constant in space and time, therefore, independent of all coordinates and $M$ is the average Mach number of the mean flow. 


\subsection{Knowledge based preliminary design}

\subsubsection{Introduction}

Scientific theories enhance our capability to describe, explain, and predict phenomena and to design artifacts that can be used to treat problems [2]. In design artifacts, one of the simple combination of above states is the half blade attaches to the mandrel shown in Fig. 3.4 and the radial wave number is written in Table 3.3.

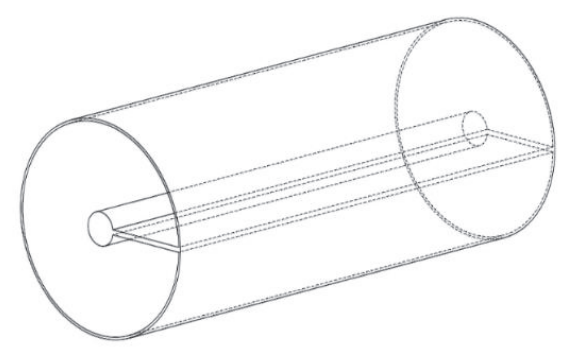

Figure 3.4: The half blade attached to the mandrel inside the duct.

Table 3.3: Radial wave number of the half blade attached to the mandrel.

\begin{tabular}{|l|c|c|}
\hline \multicolumn{2}{|c|}{$m=0.5$} \\
\hline $\mathbf{q}=\mathbf{2 . 0 8 3 3}$ & First root & Second root \\
\hline $\mathbf{q = 4 . 1 6 6 7}$ & 0.6565 & 5.6009 \\
\hline $\mathbf{q = 6 . 2 5}$ & 0.8583 & 4.1781 \\
\hline $\mathbf{q = 1 2 . 5}$ & 0.9075 & 4.0006 \\
\hline
\end{tabular}

\subsubsection{Helicoidal Resonator (HR)}

To decrease the wave number, one of the smart innovations is to install the helicoidal shape inside the duct shown in Fig. 3.5. The helicoidal shape is made by turning the blade by an axially dependent angle like $\varphi=\frac{2 \pi}{L} z$.

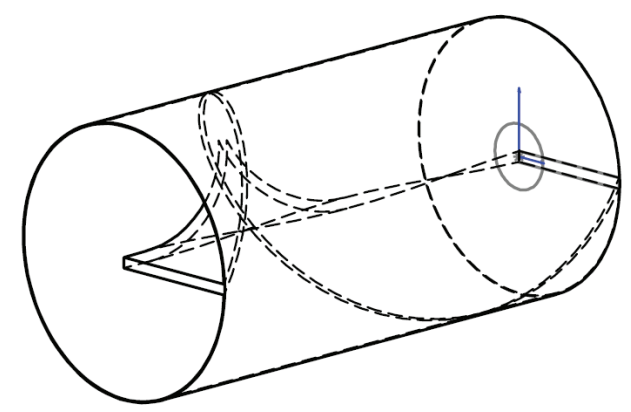

Figure 3.5: The helicoidal resonator. 
The helicoidal resonator (HR) has a boundary condition at the combination of the axial and angular axes. The pressure gradient perpendicular to the helical surface should be zero:

$$
\frac{\nabla \vec{P}}{\rho} \hat{n}=0 \Rightarrow \nabla \vec{P} . \hat{n}=0 .
$$

The pressure gradient in cylindrical coordinate could be written as below:

$$
\nabla \vec{P}=\left(\begin{array}{c}
\frac{\partial R}{\partial r} \cos (\phi)-\frac{1}{r} \frac{\partial \Phi}{\partial \phi} \sin (\phi) \\
\frac{\partial R}{\partial r} \sin (\phi)+\frac{1}{r} \frac{\partial \Phi}{\partial \phi} \cos (\phi) \\
\frac{\partial Z}{\partial z}
\end{array}\right) .
$$

In addition, the helicoid surface and a normal vector of the surface are exposed in Fig. 3.6 and it can be expressed in Eq. (3.33):

$$
\text { Helicoid Surface }=\left(\begin{array}{c}
r \cos \left(\frac{2 \pi}{L} z\right) \\
r \sin \left(\frac{2 \pi}{L} z\right) \\
z
\end{array}\right), \hat{n}=\left(\begin{array}{c}
\sin \left(\frac{2 \pi}{L} z\right) \\
-\cos \left(\frac{2 \pi}{L} z\right) \\
\frac{2 \pi r}{L}
\end{array}\right) .
$$

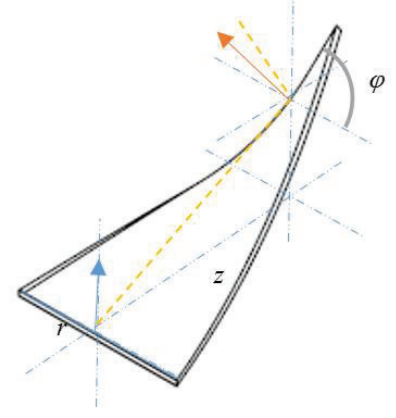

Figure 3.6: The helicoid surface.

More information can be found in Appendix A. Hence, the helicoid condition is:

$$
\begin{aligned}
& \nabla \vec{P} . \hat{n}=-\frac{1}{r} \frac{\partial \Phi}{\partial \phi}+\frac{2 \pi r}{L} \frac{\partial Z}{\partial z}=0 \\
& \Rightarrow-\frac{m}{r}\left[-A_{1 \phi} \sin (m \phi)+A_{2 \phi} \cos (m \phi)\right]+\frac{2 \pi r k_{z} i}{L}\left[-A_{1 z} e^{-i k_{z} z}+A_{2 z} e^{i k_{z} z}\right]=0
\end{aligned} .
$$

This helicoid condition is valid at $z=0, \phi=0$, so:

$$
A_{2 \phi}=\frac{2 \pi r^{2} k_{z} i}{m L}\left[-A_{1 z}+A_{2 z}\right], A_{1 \phi r}=\sqrt{1-A_{2 \phi r}^{2}} .
$$


Also, the helicoid condition is valid at $z=L, \phi=2 \pi$ :

$$
\begin{aligned}
& -\frac{m}{r}\left[-A_{1 \phi} \sin (m 2 \pi)+A_{2 \phi} \cos (m 2 \pi)\right]+\frac{2 \pi r k_{z} i}{L}\left[-A_{1 z} e^{-i k_{z} L}+A_{2 z} e^{i k_{z} L}\right]=0 \\
& \Rightarrow A_{1 \phi} \sin (m 2 \pi)=\frac{2 \pi r^{2} k_{z} i}{m L}\left[-A_{1 z} \cos (m 2 \pi)+A_{2 z} \cos (m 2 \pi)+A_{1 z} e^{-i k_{z} L}-A_{2 z} e^{i k_{z} L}\right]
\end{aligned}
$$

In Eq. (3.36), if $r=0$ then $m$ will be:

$$
\text { if } r=0 \Rightarrow \sin (m 2 \pi)=0 \Rightarrow m= \pm 0.5, \pm 1, \ldots .
$$

By substituting $m$ in Eq. (3.24), the radial wave number $k_{r}$ will be determined which is depicted earlier in Table 3.1. Moreover, Eq. (3.36) is valid at $r=r_{2}$ :

$$
\text { if } r=r_{2} \Rightarrow \frac{2 \pi r_{2}{ }^{2} k_{z} i}{m L}\left[A_{1 z}\left(1+e^{-i k_{z} L}\right)-A_{2 z}\left(1+e^{i k_{z} L}\right)\right]=0 \Rightarrow \frac{A_{1 z}}{A_{2 z}}=\frac{1+e^{i k_{2} L}}{1+e^{-i k_{z} L}}
$$

To find the maximum TL, a below equation should be valid. Hence:

$$
d(T L)=0 \Rightarrow \frac{Z_{0}}{d Z_{0}}=\frac{Z_{L}}{d Z_{L}} \Rightarrow \frac{A_{1 z}+A_{2 z}}{-A_{1 z}+A_{2 z}}=\frac{A_{1 z} e^{-i k_{z} L}+A_{2 z} e^{i k_{z} L}}{-A_{1 z} e^{-i k_{z} L}+A_{2 z} e^{i k_{z} L}}
$$

So, the axial wave number for maximum TL will be derived by considering Eq. (3.38) and (3.39).

In short, the eigen-frequency of HR which has a maximum TL is $f=\frac{k_{0} \times c_{0}}{2 \pi}, k_{0}=\left(k_{z}{ }^{2}+k_{r}{ }^{2}\right)^{0.5}$ where the axial wave number is derived from Eq. (3.39) and the radial wave number can be obtained from Table. 3.1 and Table. 3.3. 


\subsubsection{Spiral Resonator (SR)}

To absorb low frequency noise, another possibility is applying a spiral blade inside the duct as shown in Fig. 3.7.
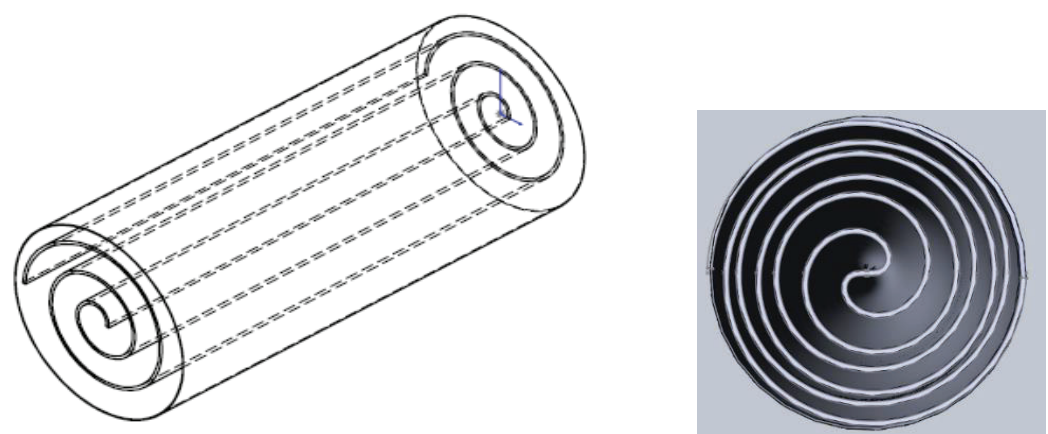

Figure 3.7: The Spiral Resonator (SR) and front view of the double spiral Resonator.

If we combine two spiral models together, the double spiral resonator presented in Fig. 3.7 is made. To find an analytical solution for the spiral resonator, it should be mentioned that the angular boundary condition will be changed and related to the function of the spiral curve. By considering the linear function, $\varphi(r)=2 n \pi \frac{r}{r_{2}}$, shown in Fig. 3.8, the solution can be derived.

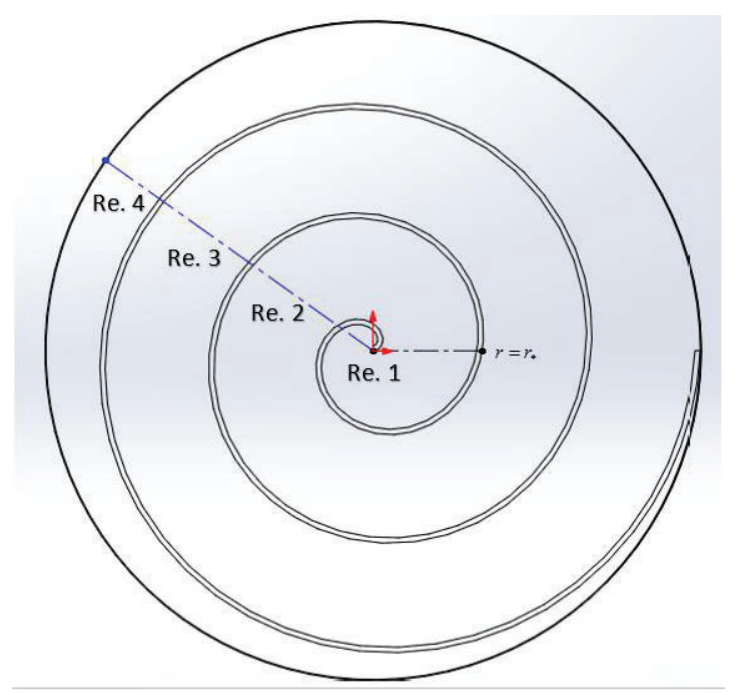

Figure 3.8: The front section view of the spiral resonator.

First, the function and normal vector of the spiral surface can be expressed (see Appendix A): 


$$
\text { Spiral Surface }=\left(\begin{array}{c}
\frac{r_{2} \phi}{2 n \pi} \cos (\phi) \\
\frac{r_{2} \phi}{2 n \pi} \sin (\phi) \\
z
\end{array}\right), \hat{n}=\left(\begin{array}{c}
\frac{r_{2}}{2 n \pi} \sin (\phi)+\frac{r_{2} \phi}{2 n \pi} \cos (\phi) \\
-\frac{r_{2}}{2 n \pi} \cos (\phi)+\frac{r_{2} \phi}{2 n \pi} \sin (\phi) \\
0
\end{array}\right) .
$$

Second, the pressure gradient perpendicular to the spiral surface should be zero. Hence:

$$
\Rightarrow \frac{\partial R}{\partial r} * r \phi-\frac{\partial \Phi}{\partial \phi}=0 \Rightarrow\left\{\begin{array}{c}
k_{r} r \phi\left[A_{m} J_{m}^{\prime}\left(k_{r} r\right)\right]-m\left[-A_{1 \phi} \sin (m \phi)+A_{2 \phi} \cos (m \phi)\right]=0 \quad r \leq r_{*} \\
k_{r} r \phi\left[A_{m} J_{m}^{\prime}\left(k_{r} r\right)+B_{m} Y_{m}^{\prime}\left(k_{r} r\right)\right]-m\left[-A_{1 \phi} \sin (m \phi)+A_{2 \phi} \cos (m \phi)\right]=0 \quad r>r_{*}
\end{array}\right.
$$

The spiral condition should be valid at $r=0, \phi=0$, so:

$$
\nabla \vec{P} . \hat{n}(r=0, \varphi=0)=0 \Rightarrow A_{2 \varphi}=0, A_{1 \varphi}=1 .
$$

From the radial boundary condition at the end of the first turn $\left(r=r_{*}, \varphi=2 \pi\right)$, we have:

$$
\frac{d R_{m}}{d r}\left(r_{*}\right)=0 \Rightarrow J_{m}^{\prime}\left(k_{r} r_{*}\right)=0
$$

The spiral condition should be also valid at the end of the first turn $\left(r=r_{*}, \varphi=2 \pi\right)$, hence:

$$
\nabla \vec{P} . \hat{n}\left(r=r_{*}, \varphi=2 \pi\right)=0 \Rightarrow \sin (m 2 \pi)=0 \Rightarrow m=(0, \pm 0.5, \pm 1, \ldots) .
$$

So, $k_{r}$ can be obtained from $J_{0.5}^{\prime}\left(k_{r} r_{*}\right)=0$. Now based on spiral condition, Eq. (3.40), the coefficient of the radial functions for the first region, Re. 1 in Fig. 3.8, will be calculated as:

$$
A_{m 1}(r)=-\frac{m \sin \left(m \frac{2 \pi r}{r_{*}}\right)}{k_{r} r \frac{2 \pi r}{r_{*}} J_{m}^{\prime}\left(k_{r} r\right)}, m=0.5 \quad 0<r \leq r_{*} .
$$

For the other regions, i.e., 2, 3 and 4, it can be found in Appendix A.

If both ends of the spiral resonator are opened, $Z(z)=\cos \left(k_{z} z\right)$, the pressure at the inlet will be the same as at the outlet and no noise reduction will be obtained. To overcome this difficulty, one of the solutions is to close a part of each of the ends like in Fig. 3.9: 


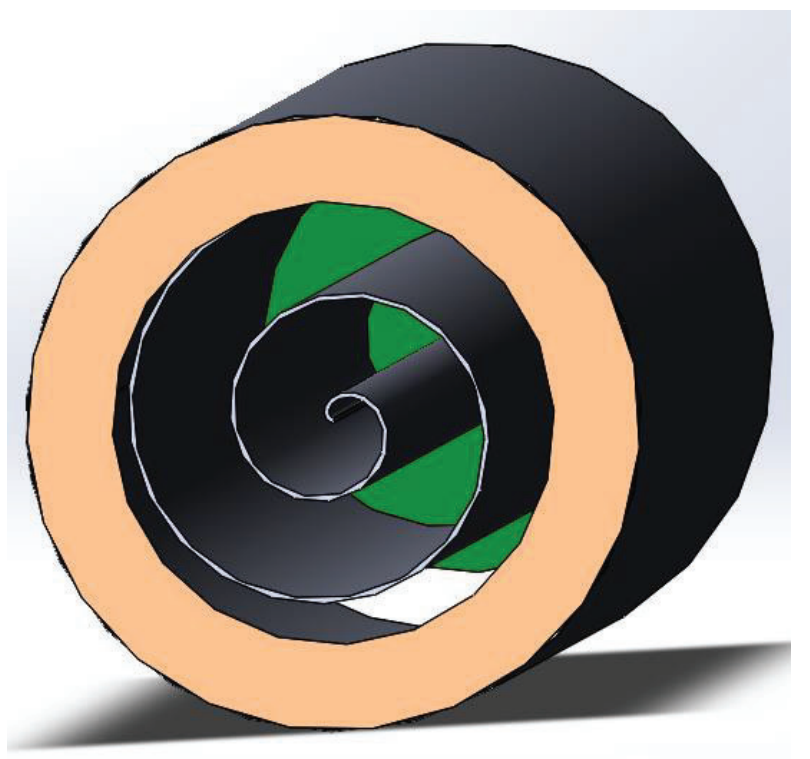

Figure 3.9: The spiral resonator with half closed ends.

In this case, the axial boundary condition will be changed at the closed end parts, so:

$$
\left\{\begin{array}{ccc}
Z(z=L)=0 \Rightarrow A_{1 z} e^{-i k_{z} L}+A_{2 z} e^{i k_{z} L}=0 & r \leq r_{0} \quad \text { (half outlet is closed) } \\
Z(z=0)=0 \Rightarrow A_{1 z} e^{-i k_{z} 0}+A_{2 z} e^{i k_{z} 0}=0 & r>r_{0} & \text { (half inlet is closed) }
\end{array} .\right.
$$




\subsubsection{Funnel Resonator (FR)}

The other method to reduce the low frequency noise is using a funnel inside the tube which is demonstrated in Fig. 3.10. The axial and radial wave number will be derived from below formula.

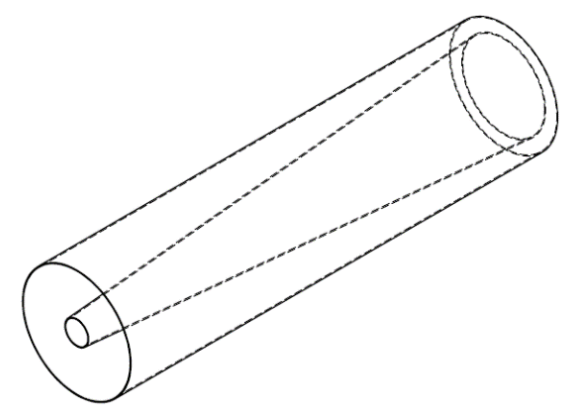

Figure 3.10: The funnel resonator inside the duct.

The funnel surface and normal vector of the surface can be expressed:

$$
\text { Funnel Surface }=\left(\begin{array}{c}
\left(r_{1}+\frac{r_{2}-r_{1}}{L} z\right) \cos (\phi) \\
\left(r_{1}+\frac{r_{2}-r_{1}}{L} z\right) \sin (\phi) \\
z
\end{array}\right), \hat{n}=\left(\begin{array}{c}
\left(\frac{L}{r_{2}-r_{1}} r_{1}+z\right) \cos (\phi) \\
\left(\frac{L}{r_{2}-r_{1}} r_{1}+z\right) \sin (\phi) \\
-\left(\frac{L}{r_{2}-r_{1}} r_{1}+z\right)
\end{array}\right) \text {. }
$$

The pressure gradient perpendicular to the funnel surface should be zero, hence:

$$
\nabla \vec{P} . \hat{n}=\left(\frac{L}{r_{2}-r_{1}} r_{1}+z\right)\left[\frac{\partial R}{\partial r}-\frac{\partial Z}{\partial z}\right]=0 \Rightarrow \frac{\partial R}{\partial r}-\frac{\partial Z}{\partial z}=0 .
$$

The funnel condition should be valid at funnel surface inside and outside of the funnel for $z=0$ and $z=L$ :

$$
\text { inside of the funnel } \Rightarrow\left\{\begin{array}{l}
J_{m}^{\prime}\left(k_{r 01} r_{01}\right)=\frac{i k_{z 01}}{k_{r 01}}\left[-A_{1 z} e^{-i k_{z 01} 0}+A_{2 z} e^{i k_{z 01} 0}\right], \quad k_{z 01}^{2}=k^{2}-k_{r 01}^{2} \\
J_{m}^{\prime}\left(k_{r L 1} r_{L 1}\right)=\frac{i k_{z L 1}}{k_{r L 1}}\left[-A_{1 z} e^{-i k_{z L 1} L}+A_{2 z} e^{i k_{z L 1} L}\right], \quad k_{z L 1}^{2}=k^{2}-k_{r L 1}^{2}
\end{array} .\right.
$$

By solving above 2 equations, 2 unknown parameters $k_{r 01}, k_{r L 1}$ can be found. The funnel condition should also be valid at the outside region of the funnel for $z=0$ and $z=L$ : 
outside of the funnel $\Rightarrow\left\{\begin{array}{l}{\left[A_{m 1} J_{m}^{\prime}\left(k_{r 02} r_{01}\right)+B_{m 1} Y_{m}^{\prime}\left(k_{r 02} r_{01}\right)\right]=\frac{i k_{z 02}}{k_{r 02}}\left[-A_{1 z} e^{-i k_{z 02} 0}+A_{2 z} e^{i k_{z 02} 0}\right]} \\ {\left[A_{m 2} J_{m}^{\prime}\left(k_{r L 2} r_{L 1}\right)+B_{m 2} Y_{m}^{\prime}\left(k_{r L 2} r_{L 1}\right)\right]=\frac{i k_{z L 2}}{k_{r L 2}}\left[-A_{1 z} e^{-i k_{z L 2} L}+A_{2 z} e^{i k_{z L 2} L}\right]}\end{array}\right.$.

The duct Boundary condition will give:

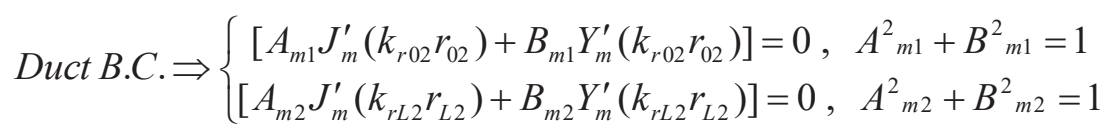

By solving the above 6 equations, 6 unknown parameters $2 k_{r}, A_{m 1}, B_{m 1}, A_{m 2}, B_{m 2}$ can be found. It means that when the acoustic pressure field can be obtained by these parameters then the transmission loss is derived. For instance, consider that a FR with $0.6 \mathrm{~m}$ length is installed in a duct with $0.125 \mathrm{~m}$ diameter and $d_{0}=0.03 \mathrm{~m}, d_{L}=0.055 \mathrm{~m}$, this model will be compared in next chapters, based on written MATLAB code the first five frequencies are 287, $578,858,1147,1455 \mathrm{~Hz}$.

\subsubsection{Spiral-Helicoidal Resonator (SHR)}

The combination of spiral and helicoidal resonators will give a new resonator, shown in Fig. 3.11, which has lower eigen-frequencies whereas, with the helicoidal resonator, these frequencies cannot be achieved.

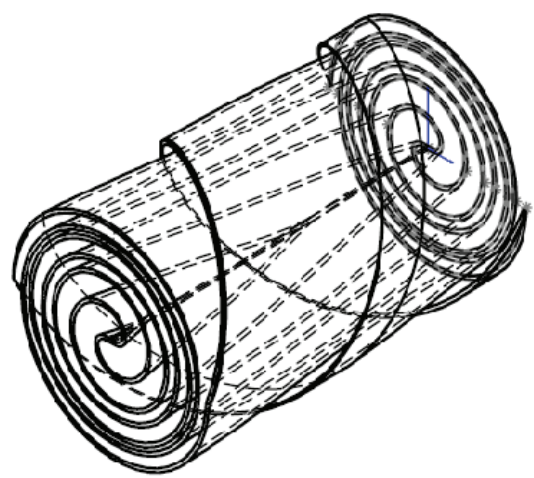

Figure 3.11: The Spiral-Helicoidal Resonator (SHR).

One of the advantages of SHR is that by rotating only $61^{\circ}$ instead of $360^{\circ}$, displayed in Fig. 3.12 , the desired eigen-frequencies could be achieved with less pressure drop. 


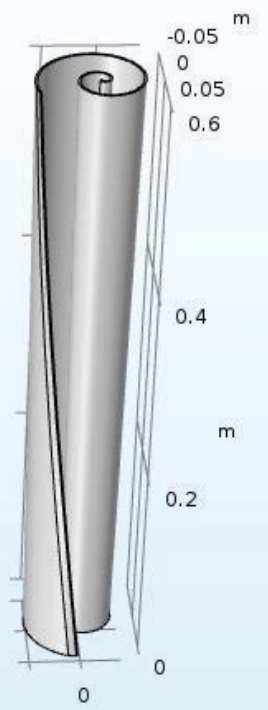

Figure 3.12: The spiral-helicoidal resonator with $61^{\circ}$ helical rotation.

\subsubsection{Funnel-Spiral-Helicoidal Resonator (FSHR)}

The other combination of these three resonators termed Funnel-Spiral-Helicoidal Resonator (FSHR) is shown in Fig. 3.13. Based on the simulation results, this model has more eigenfrequencies lower than $1000 \mathrm{~Hz}$ and it has less pressure drop while the making process will become more complicated.

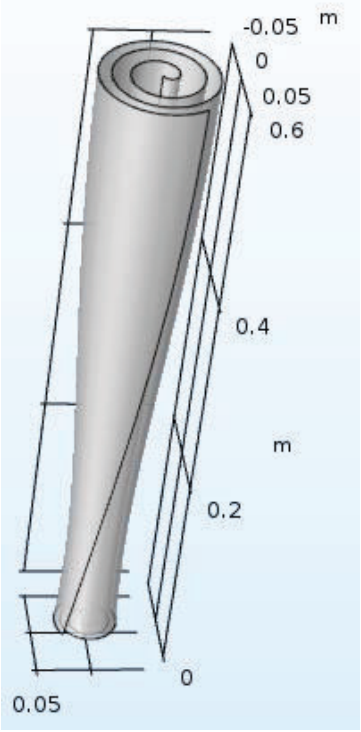

Figure 3.13: The funnel-spiral-helicoidal resonator. 


\subsubsection{Acoustic Black Hole effect}

The Acoustic Black Hole ( $A B H)$ can be considered as a passive treatment for low frequency noise reduction inside the duct. A smooth decrease in the velocity of wave propagation in finite length is the main principle of the $A B H$. The $A B H$ includes a specified number of tapered edges with particular distances from each other assembled inside a tube. The plates could be set on parallel form termed Normal Acoustical Black Hole (NABH) and a spiral tapered edge could also be used which is termed Spiral Acoustic Black Hole (SABH). These tapered edges should be smooth enough to cause no reflection by itself; also, the sound propagation inside the $\mathrm{ABH}$ will take extremely long time to reach the other part of the $\mathrm{ABH}$, which is causing zero reflection therefore. The function of the inner diameter of the blades might be linear or quadratic, which are termed LABH and QABH respectively [19]. As mentioned before, the linear Euler's equation is [11]:

$$
-\nabla p=\left(\rho_{0} \frac{\partial V}{\partial t}\right)
$$

where $V(x, y, z)$ is the fluid velocity at position $(x, y, z)$. In case of a narrow, axially symmetric waveguide of varying cross section, this equation can be written as:

$$
-\frac{\partial p}{\partial z}=\left(\rho_{0} \frac{\partial u}{\partial t}\right)
$$

where $u$ is the velocity component on the $z$ axis. For varying cross section, the equation of continuity can be written as [19]:

$$
d\left(\rho_{0} S u\right)+\left(2 \pi r v \rho_{0}\right) d z+\frac{\partial \rho}{\partial t} S d z=0
$$

where $S=S(z)$ and $v$ is the projection of the velocity of fluid near the walls the perpendicular to the symmetry axis. The boundary admittance is defined as the quotient of the relative particle velocity and the sound pressure at the boundary [20], i.e.,

$$
Y(z)=\frac{v_{r e l}(z)}{p(z)},
$$


where the relative particle velocity is defined as the difference between the particle velocities of the fluid and the structure. The reciprocal value is well known as the boundary impedance, defined as:

$$
Z(z)=\frac{p(z)}{v_{r e l}(z)}=\frac{1}{Y(z)} .
$$

For many applications, it is useful to introduce dimensionless boundary admittances and impedances defined as [20]:

$$
\tilde{Y}(z)=\rho c Y \quad \text { and } \quad \tilde{Z}(z)=\frac{Z}{\rho c}=\frac{1}{\tilde{Y}(z)} .
$$

Substituting wall admittance in the equation of continuity, we obtain [19]:

$$
-\frac{1}{\rho_{0}} \frac{\partial \rho}{\partial t}=u(\ln S)_{z}^{\prime}+\frac{\partial u}{\partial z}+\frac{2 Y p}{r}
$$

Taking into account the equality $\frac{\partial \rho}{\partial t}=\frac{1}{c^{2}} \frac{\partial p}{\partial t}$, the wave equation for the pressure will be [19]:

$$
-\frac{1}{c^{2}} \frac{\partial^{2} p}{\partial t^{2}}+\frac{2 Y \rho_{0}}{r} \frac{\partial p}{\partial t}=\frac{\partial^{2} p}{\partial z^{2}}+\frac{\partial p}{\partial z}(\ln S)_{z}^{\prime} .
$$

Assuming a time harmonic dependence of the acoustic pressure, $p(r, \phi, z, t)=p(r, \phi, z) e^{j \omega t}$, the equation reads in the frequency domain:

$$
\frac{\partial^{2} p}{\partial z^{2}}+\frac{\partial p}{\partial z} \frac{\partial(\ln S)}{\partial z}+p\left[k_{0}{ }^{2}+j \omega \frac{2 Y}{r}\right]=0,
$$

where $k_{0} \equiv \frac{\omega}{c_{0}}$ is a wave number. It should be noted that for zero-valued admittance $Y$, this equation transforms to the Webster equation. By considering the linear Euler's equation, the fluid particle velocity is related to the sound pressure by [20]:

$$
v=\frac{\nabla p}{j \rho_{0} \omega}
$$




\subsubsection{Acoustic Black Hole muffler}

The first model of Acoustic Black Hole muffler shown in Fig. 3.14 is proposed by Mironov [19].

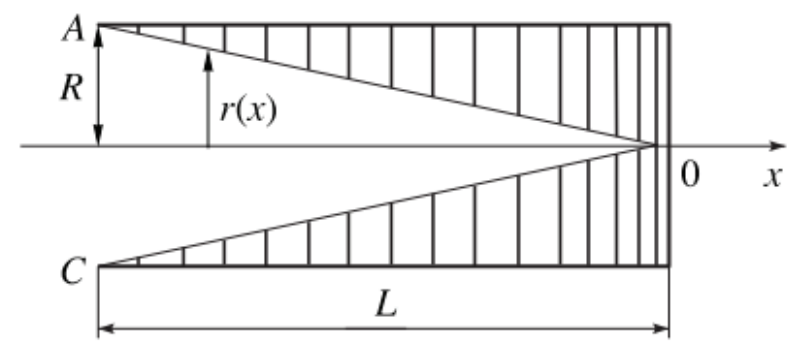

Figure 3.14: Acoustic Black Hole muffler.

In this model the linear radius function is expressed as:

$$
r_{2 x}=-\frac{R}{L} x \text { and } R=r_{1}
$$

Hence, if the losses are neglected, the admittance in cylindrical coordinate would be [19]:

$$
Y\left(r=r_{z}, \varphi, z\right)=(-j \omega) \frac{1}{\rho_{0} c^{2}} \frac{R^{2}-r_{z}{ }^{2}}{2 r_{z}} .
$$

Substituting the above equation into the wave equation for the pressure, and taking into account that $S=\pi r^{2}$, result in [19]:

$$
\frac{\partial^{2} p}{\partial z^{2}}+2 \frac{\partial p}{z \partial z}+p\left[\frac{k_{0}{ }^{2} R^{2}}{r^{2}}\right]=0 .
$$

For the linear Acoustic Black Hole muffler, this equation has a set of exact particular solutions in the form of power-law functions [19]:

$$
p(z)=A_{\alpha}(z / L)^{\alpha} \quad \text { and } \quad \alpha_{1,2}=-\frac{1}{2} \pm \sqrt{\frac{1}{4}-\left(k_{0} L\right)^{2}} .
$$

Due to the closed end of the $\mathrm{ABH}$ muffler, it cannot be used inside the duct directly and it requires some modifications to be suitable for applying in the ventilation systems. For instance, the $\mathrm{ABH}$ muffler could be easily installed inside the T-joint to absorb the incident wave energy of the flow which is shown in Fig. 3.15. The size of the ABH muffler is suitable to apply in any bend pipe, elbows, and it has a negligible pressure drop. If the air flows into the $\mathrm{ABH}$ muffler, it might cause turbulences which results in no absorption. Therefore, the appropriate solution can include a membrane in front of the $\mathrm{ABH}$ muffler to allow only the 
sound waves go into the $\mathrm{ABH}$ muffler. In short, this method is as a reliable way to get rid of the low frequency noise inside the duct with low investment.

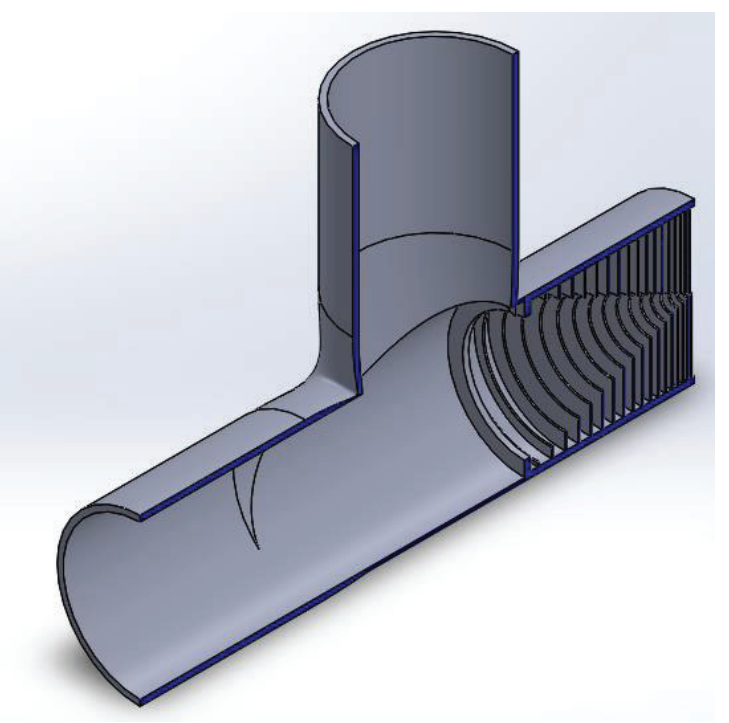

Figure 3.15: Section view of the Acoustic Black Hole muffler installing inside the T-joint.

\subsubsection{External Acoustic Black Hole muffler}

The second model of Acoustic Black Hole muffler, shown in Fig. 3.16, is termed External Acoustic Black Hole (EABH) muffler [19].

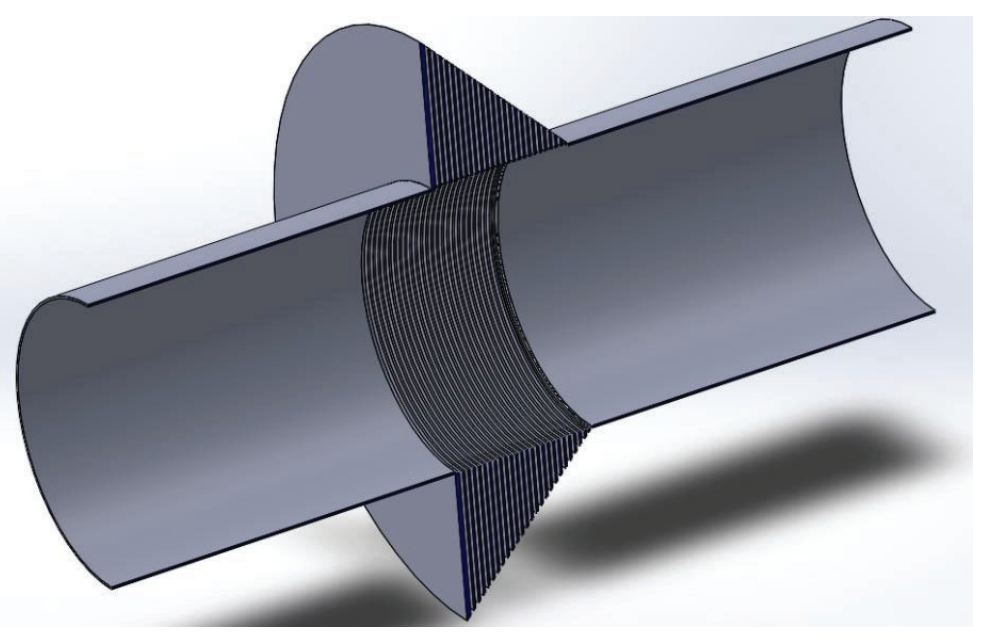

Figure 3.16: Section view of External Acoustic Black Hole muffler.

As mentioned before, the pressure could be written as $P(r, \varphi, z, t)=R(r) \Phi(\varphi) Z(z) T(t)$. So, the dimensionless admittance could be expressed as [21]: 


$$
\widetilde{Y}\left(r=r_{1}\right)=\frac{\rho c}{j \rho_{0} \omega} \frac{J_{m}^{\prime}\left(k_{r} r_{1}\right)-\frac{J_{m}^{\prime}\left(k_{r} r_{2}\right)}{Y_{m}^{\prime}\left(k_{r} r_{2}\right)} Y_{m}^{\prime}\left(k_{r} r_{1}\right)}{\left.J_{r} r_{1}\right)-\frac{J_{m}^{\prime}\left(k_{r} r_{2}\right)}{Y_{m}^{\prime}\left(k_{r} r_{2}\right)} Y_{m}\left(k_{r} r_{1}\right)}
$$

where $m=0$ due to the axisymmetric mode. In this model the linear radius function is expressed as:

$$
r_{2 x}=r_{1}+\alpha^{*} x
$$

Hence, if the losses are neglected, assuming $k_{r} r_{1}<<1$ and $k_{r} r_{2}<<1$ and making use of Taylor's expansions of Bessel function for $m=0$, see Appendix A, the wall admittance equation in cylindrical coordinate is simplified to the previous formula in Acoustics Black Hole as below, and then the solution is the same as mentioned before:

$$
Y\left(r=r_{1}\right)=\frac{\rho c k_{r} r_{1}}{j \rho_{0} \omega} \frac{\left(\frac{r_{2}}{r_{1}}\right)^{2}-1}{2} .
$$

In summary, various resonators and mufflers are introduced and governing equations are derived. The differences between HR, SR, and FR are only on their boundary conditions, i.e., the surface which installs inside the tube. These differences shift the axial and radial wave numbers that causes noise absorption at the defined frequencies. In mufflers, the length of channels are important. Moreover, the function of increasing the length of channels has a strong effect on the transmission loss of mufflers. In this chapter to find the analytical solution, models are simplified and main equations are just expressed. However, it is possible to work on the detail of each resonator and muffler. Also, it should be noted that more time is needed to adapt the solution and results with a practical result which will be discussed in next chapters. 


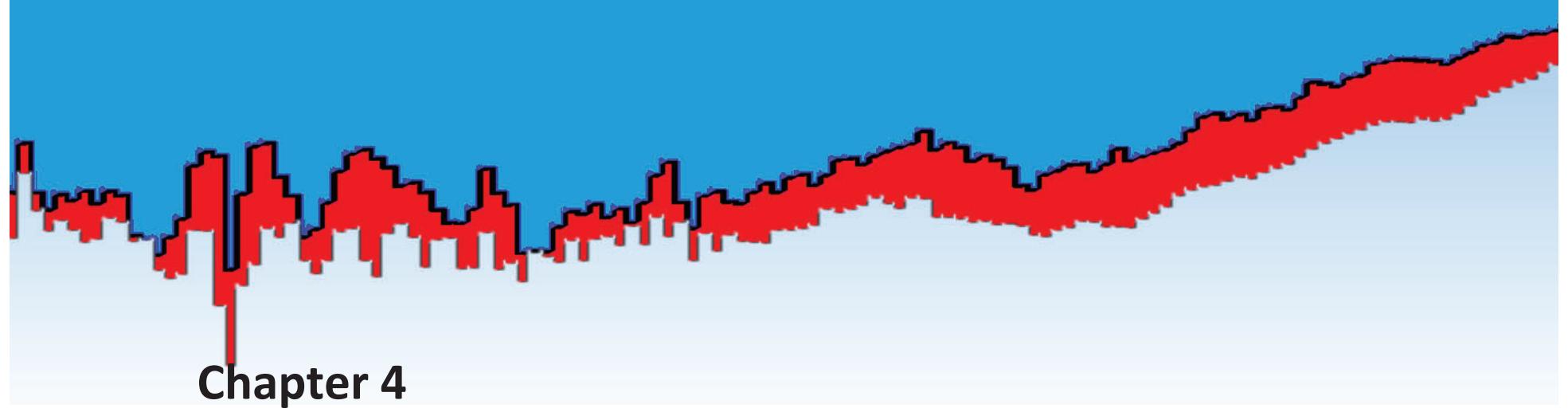

\section{Treatment Validation}

\subsection{Introduction}

In the third task of the design cycle, treatment validation phase, the noise reduction systems are designed and simulated by commercial software SolidWorks, COMSOL, and MATLAB. COMSOL Multiphysics is a simulation software and in this project pressure acoustic and solid mechanics modules of COMSOL are used. In some simulations, it is needed to solve the coupled acoustic-structure equations so a multiphysics module is applied for an acousticstructure interaction (ASI). Moreover, for taking into account losses in mufflers, a poroacoustics module with Delany-Bazley-Miki model has been applied [16]. A flow resistivity of $80 \mathrm{~Pa} . \mathrm{sec} / \mathrm{m}^{2}$ was used. The performance of the numerical results will determine the development of the first samples. This will result in the detail design and development phase where prototypes will be built and tested.

\section{2 Helicoidal Resonator}

The first model is the helicoidal resonator similar to the Łapka's model (involves the helicoidal resonator with a muffler) shown in Fig. 4.1:

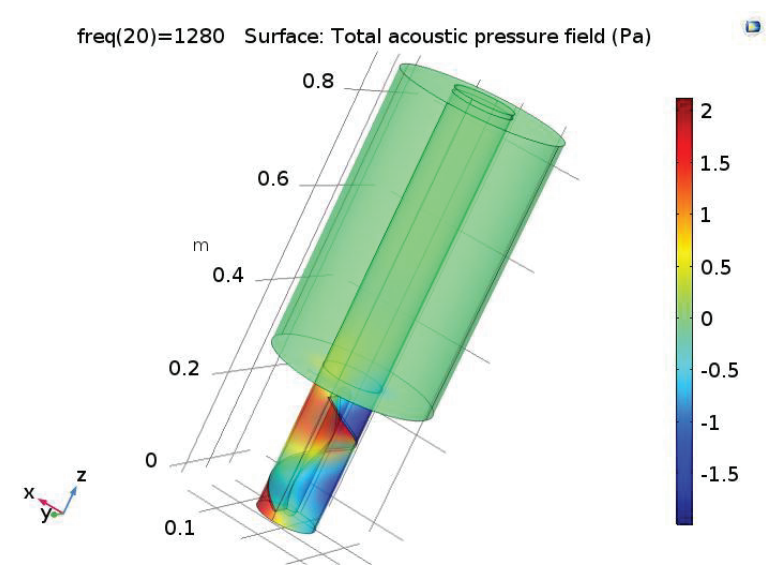

Figure 4.1: The helicoidal resonator with the muffler. 
The transmission loss of three combinations of the HR and the muffler are depicted in Fig. 4.2. To compare and validate the simulation model, the transmission loss of the Łapka's model [22] is also presented in Fig. 4.3.

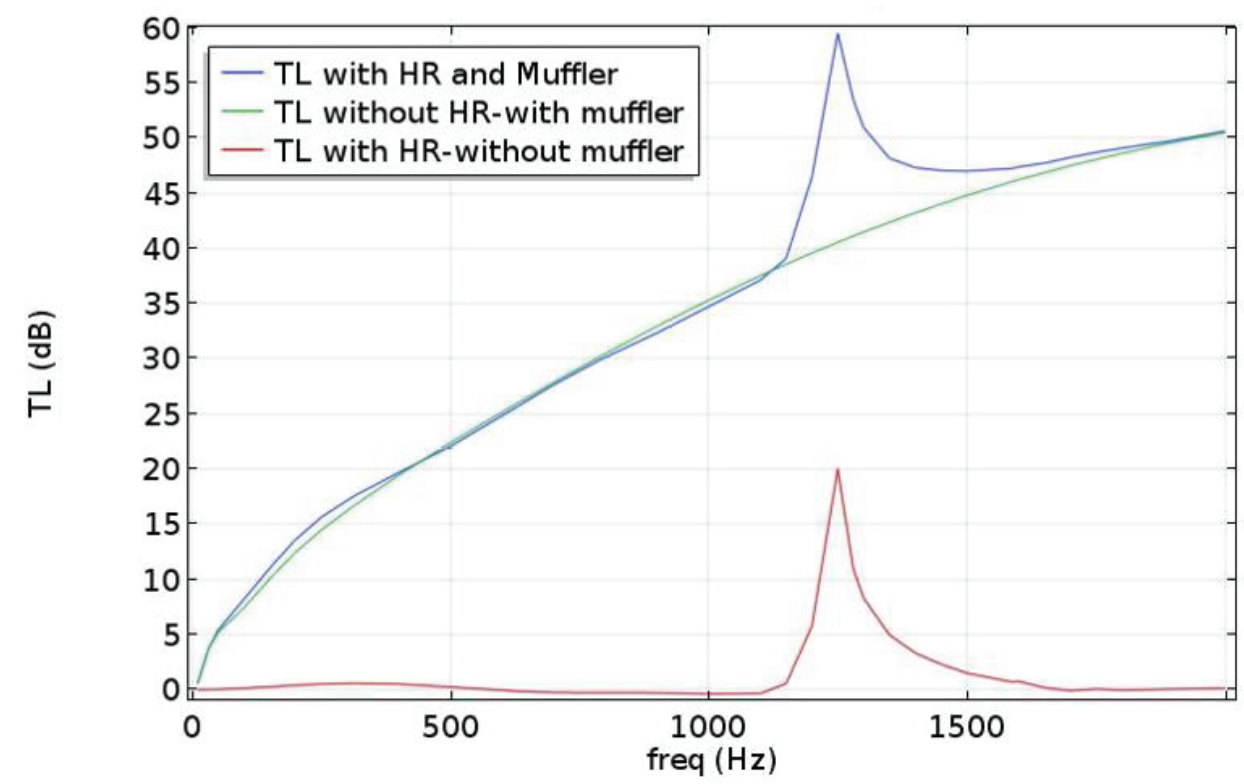

Figure 4.2: Transmission loss of the helicoidal resonator combine with a muffler.

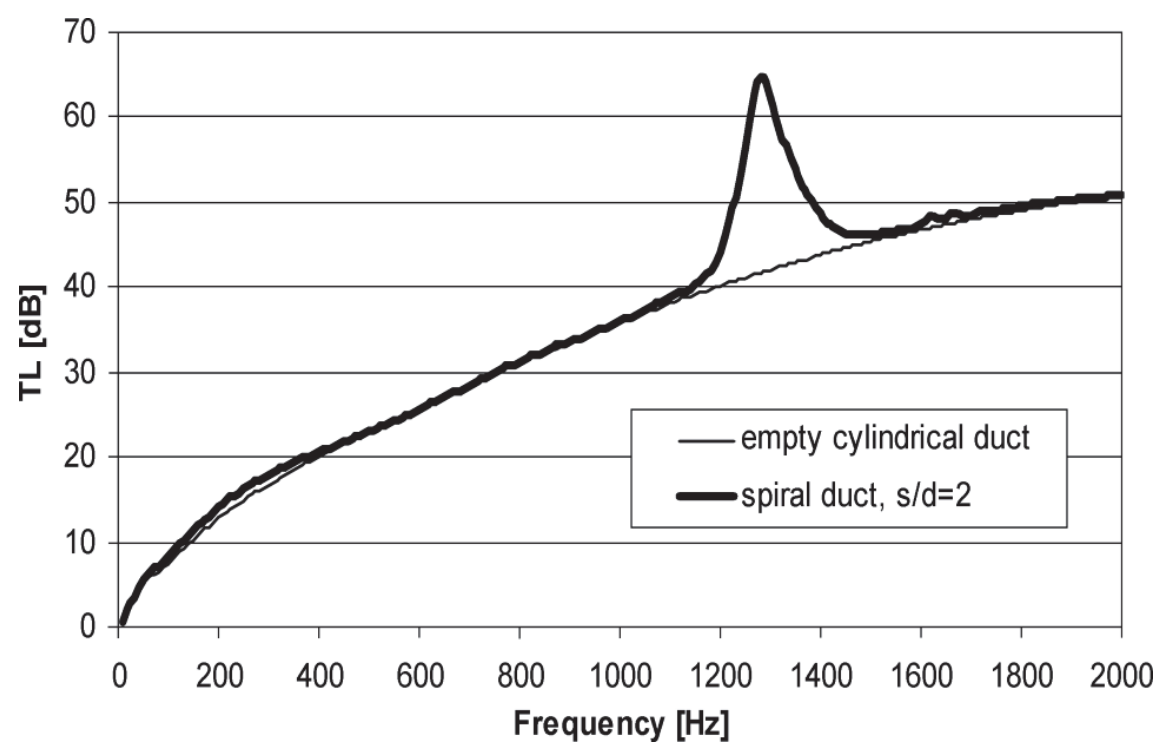

Figure 4.3: Transmission loss of the Lapka's model for the helicoidal resonator with a muffler [22].

By comparing Fig. 4.2, with Fig. 4.3, it can be concluded that the simulation models are similar which results in using this model of simulation for the other resonators. 
To find out the performance of the helicoidal resonator, the pressure acoustics of two models i.e. flat plate and helicoidal resonator inside the ducts at their first eigen-frequencies are depicted in Fig. 4.4.

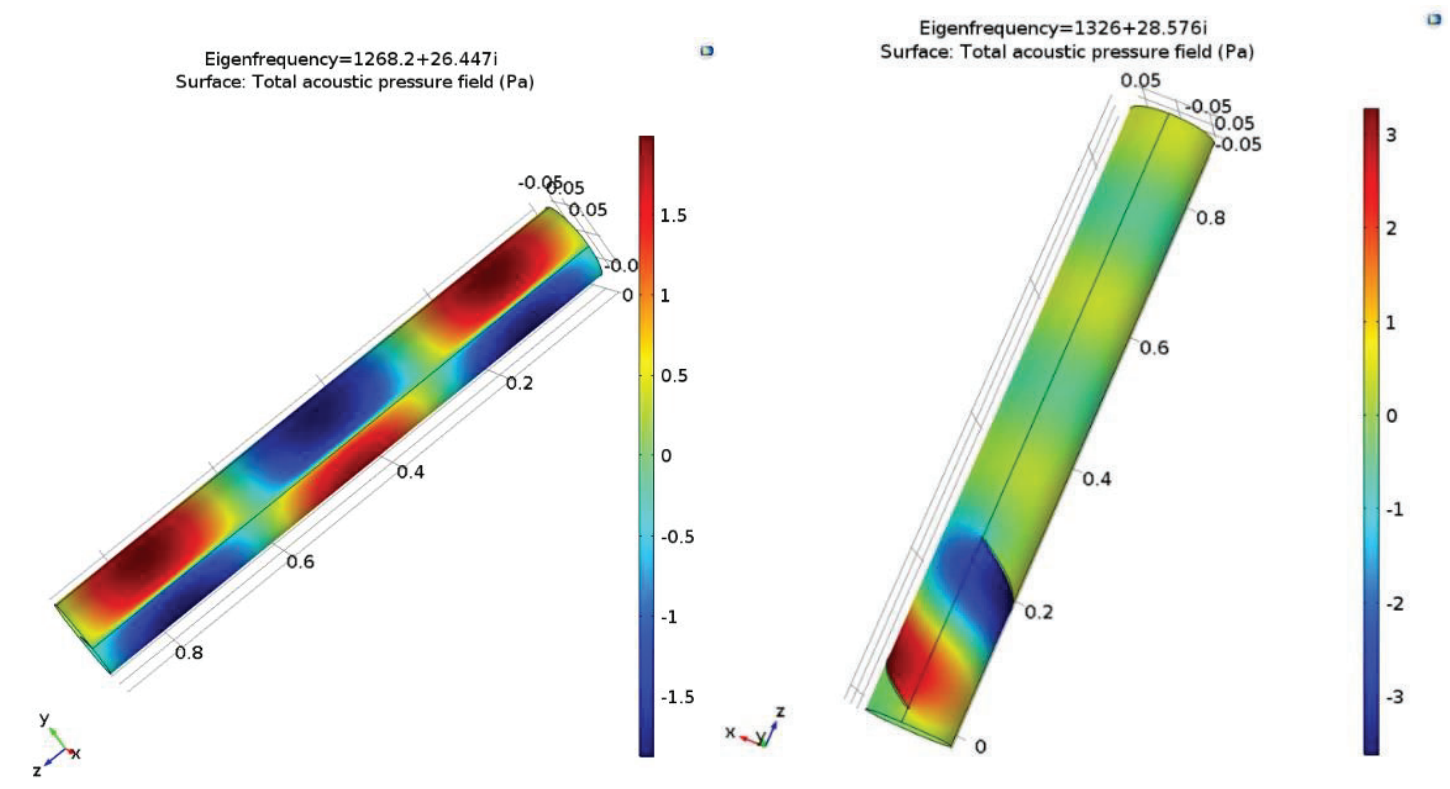

Figure 4.4. The numerical solution of inserting a flat blade and helicoidal resonator inside the duct.

As can be seen in Fig. 4.4 unlike the flat plate inside the duct, the positive pressure region of the air with the negative pressure region after the helicoidal resonator are mixed which causes noise attenuation at that first eigen-frequency. The advantage of the helicoidal resonator is to tune the noise and it cancels the noise at the first eigen-frequency. The MATLAB code based on analytical solution is written and the comparison of the COMSOL simulation's result with the analytical solution is demonstrated in Table 4.1:

Table 4.1: First eigen-frequency determined with analytical and numerical solutions.

\begin{tabular}{|c|c|c|c|}
\hline Resonator & Specification & Numerical result & Analytical Result \\
\hline $\begin{array}{l}\text { Helicoidal resonator } \\
\text { Without mandrel }\end{array}$ & $\begin{array}{l}\text { Length: } 0.247 \mathrm{~m}, \mathrm{R}=0.0625 \mathrm{~m} \\
\text { Thickness: } 5 \mathrm{~mm}\end{array}$ & $1331 \mathrm{~Hz}$ & $1335 \mathrm{~Hz}$ \\
\hline $\begin{array}{l}\text { Helicoidal resonator } \\
\text { Without mandrel }\end{array}$ & $\begin{array}{l}\text { Length: } 0.5 \mathrm{~m}, \mathrm{R}=0.0625 \mathrm{~m} \\
\text { Thickness: } 1 \mathrm{~mm}\end{array}$ & $1108 \mathrm{~Hz}$ & $1102 \mathrm{~Hz}$ \\
\hline $\begin{array}{l}\text { Helicoidal resonator } \\
\text { Without mandrel }\end{array}$ & $\begin{array}{l}\text { Length: } 0.7 \mathrm{~m}, \mathrm{R}=0.0625 \mathrm{~m} \\
\text { Thickness: } 1 \mathrm{~mm}\end{array}$ & $1066 \mathrm{~Hz}$ & $1060 \mathrm{~Hz}$ \\
\hline $\begin{array}{l}\text { Plate inside the duct } \\
\text { Without mandrel }\end{array}$ & $\begin{array}{l}\text { Length: } 0.254 \mathrm{~m}, \mathrm{R}=0.0625 \mathrm{~m} \\
\text { Thickness: } 1 \mathrm{~mm}\end{array}$ & $1244 \mathrm{~Hz}$ & $1221 \mathrm{~Hz}$ \\
\hline $\begin{array}{l}\text { Helicoidal Resonator } \\
\text { with mandrel }\end{array}$ & $\begin{array}{l}\text { Length: } 0.254 \mathrm{~m}, \mathrm{R}=0.0625 \mathrm{~m} \\
\text { Thickness: } 1 \mathrm{~mm}\end{array}$ & $1130 \mathrm{~Hz}$ & $1129 \mathrm{~Hz}$ \\
\hline
\end{tabular}




\subsection{Spiral Resonator}

One of the spiral resonators with three spiral turns and the length of $0.5 \mathrm{~m}$ which is possible to put inside the ventilation duct is simulated in this section. As mentioned before, to force the air to have spiral rotation, half part of the one ends is closed (see. Fig. 3.9). The acoustic pressure field at the first eigen-frequency of this model, $430 \mathrm{~Hz}$, is displayed in Fig 4.5:

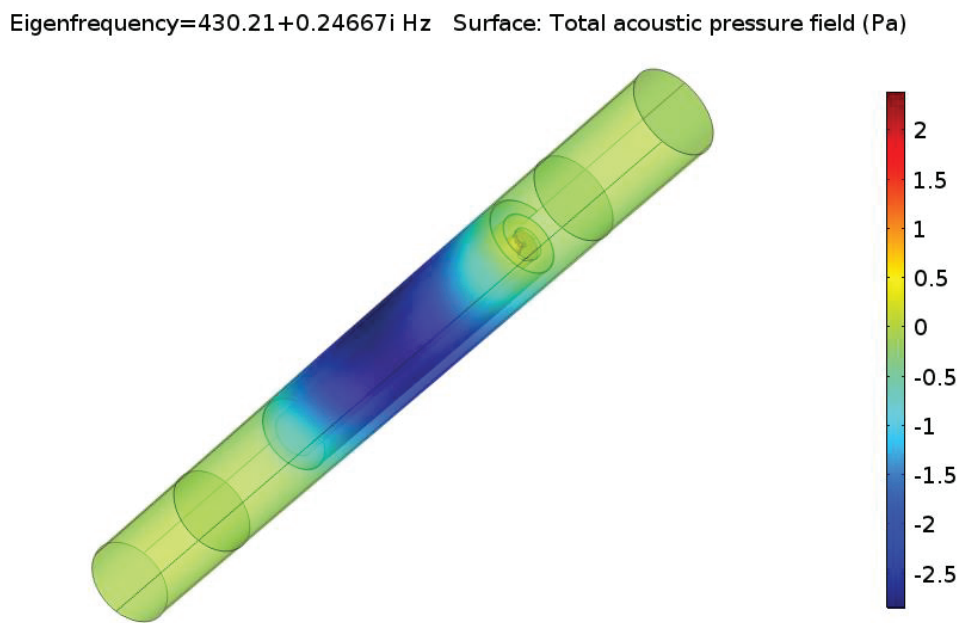

Figure 4.5: Acoustic pressure filed of the spiral resonator at first eigen-frequency.

The transmission loss of the mentioned spiral resonator at $400 \mathrm{~Hz}$ to $1150 \mathrm{~Hz}$ is shown in Fig. 4.6.

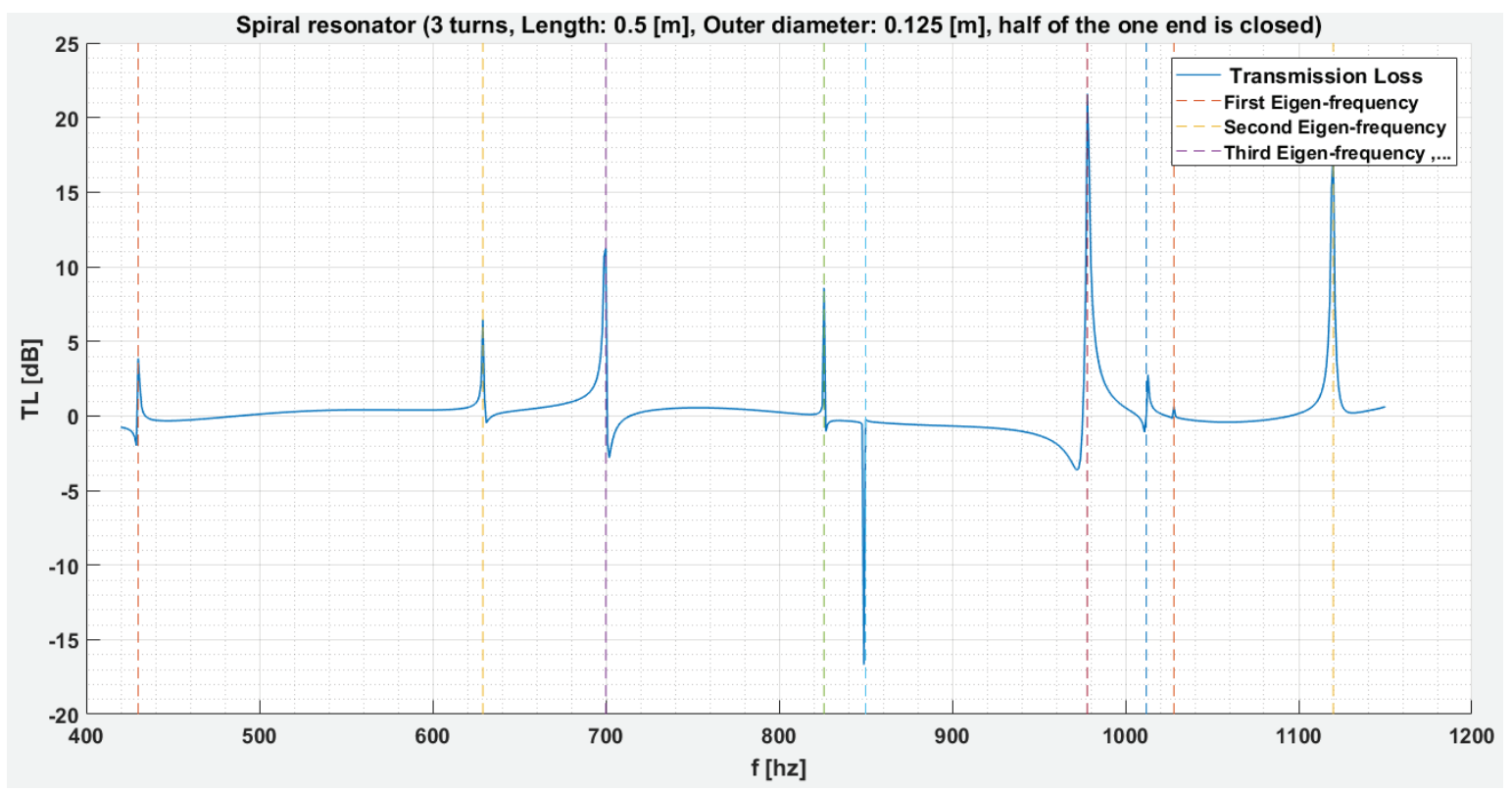

Figure 4.6: Transmission loss of the spiral resonator with three spiral turns. 
As can be seen in Fig. 4.6 the eigen-frequencies are 430, 629, 700, 826, 850, 978, 1012, 1028 $\mathrm{Hz}$. If the length of spiral resonator decreases to $0.254 \mathrm{~m}$ and half of the both ends are closed, then the first and second eigen-frequencies are shifted to the right which is shown in Fig. 4.7:

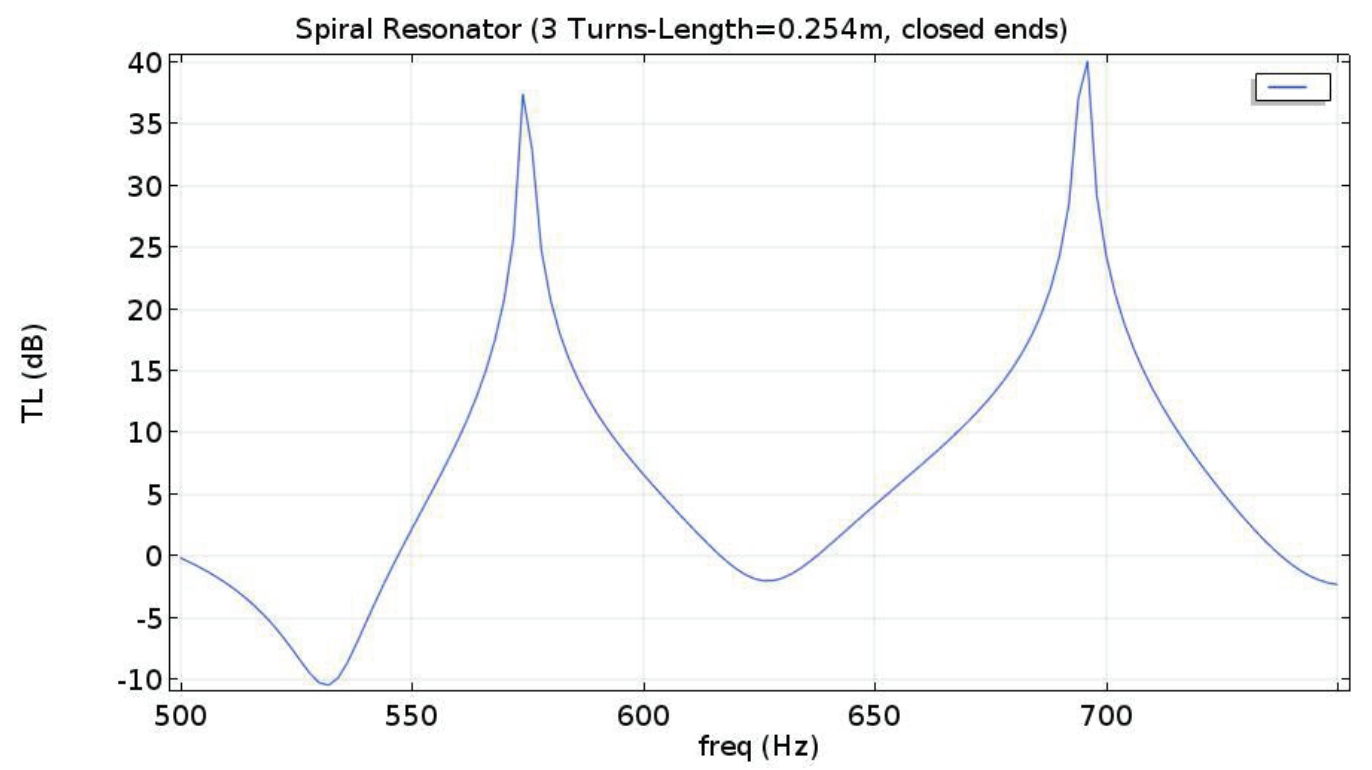

Figure 4.7: Transmission loss of the spiral resonator at the first to the second eigen-frequencies.

It should be mentioned that, using the helicoidal resonator with the same length the first eigen-frequency which caused noise attenuation was $1273 \mathrm{~Hz}$ while using the spiral resonator, it drops to $578 \mathrm{~Hz}$ that cannot be achieved by the helicoidal one anymore.

If the double spiral resonator with three spiral turns and $0.8 \mathrm{~m}$ length is used, then the TL will become as Fig. 4.8: 


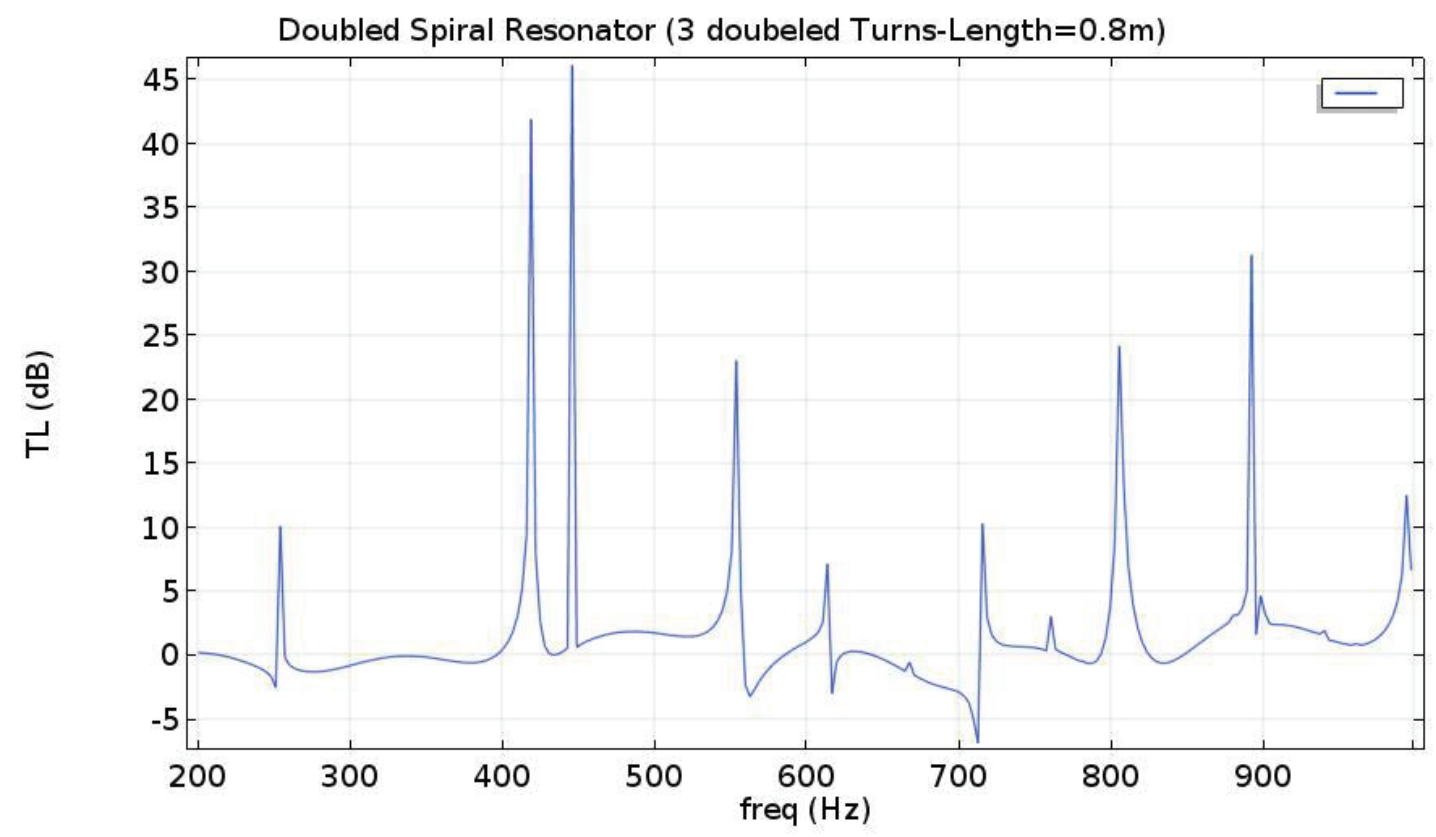

Figure 4.8: Transmission loss of the double spiral resonator.

\subsection{Funnel Resonator}

The next resonator is the funnel resonator, this resonator has simple shape, and it can absorb the noise at its low eigen-frequencies. The transmission loss of the funnel resonator with 0.5 $\mathrm{m}$ length is demonstrated in Fig. 4.9:

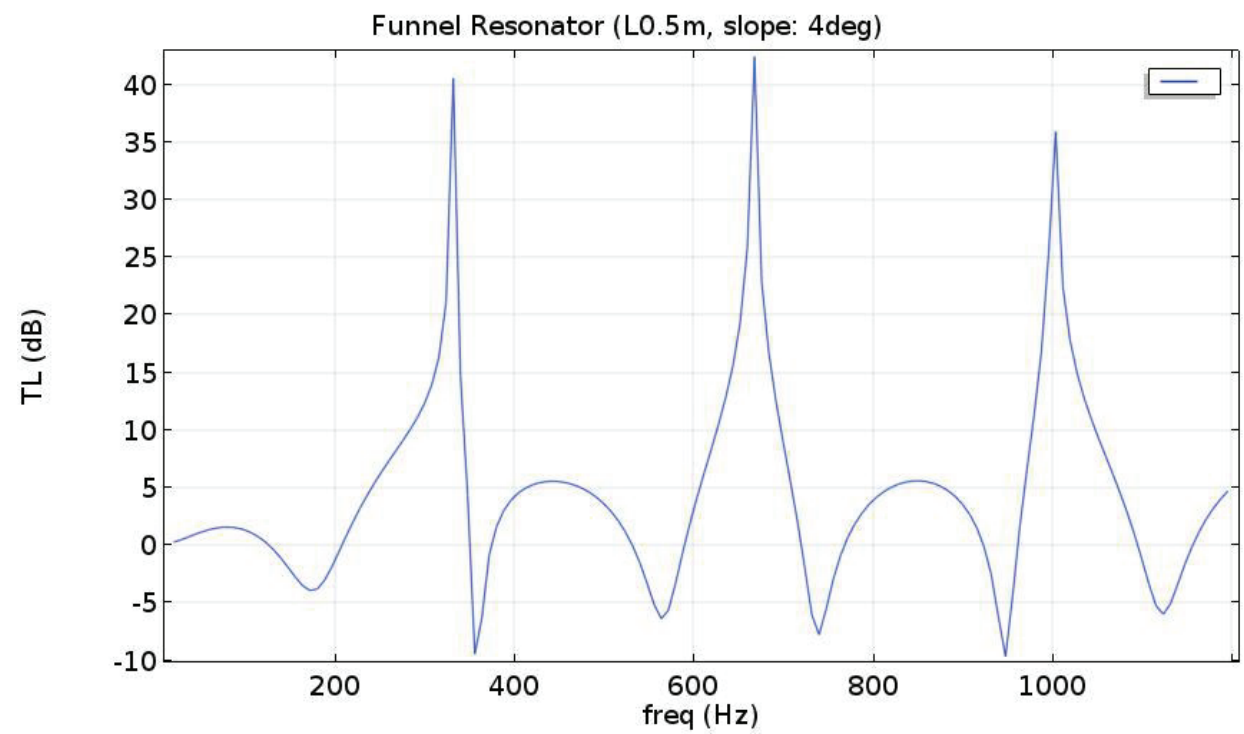

Figure 4.9: Transmission loss of the funnel resonator.

If the length of the funnel increases up to the $0.6 \mathrm{~m}$ and decreases the slope to the 2.36 degree, then the first eigen-frequency would be $272 \mathrm{~Hz}$, i.e., the critical frequency of the fan, 
which is our desired frequency. The acoustic pressure field of the FR at the first eigenfrequency and TL of this resonator are plotted in Fig. 4.10 and Fig. 4.11 respectively.

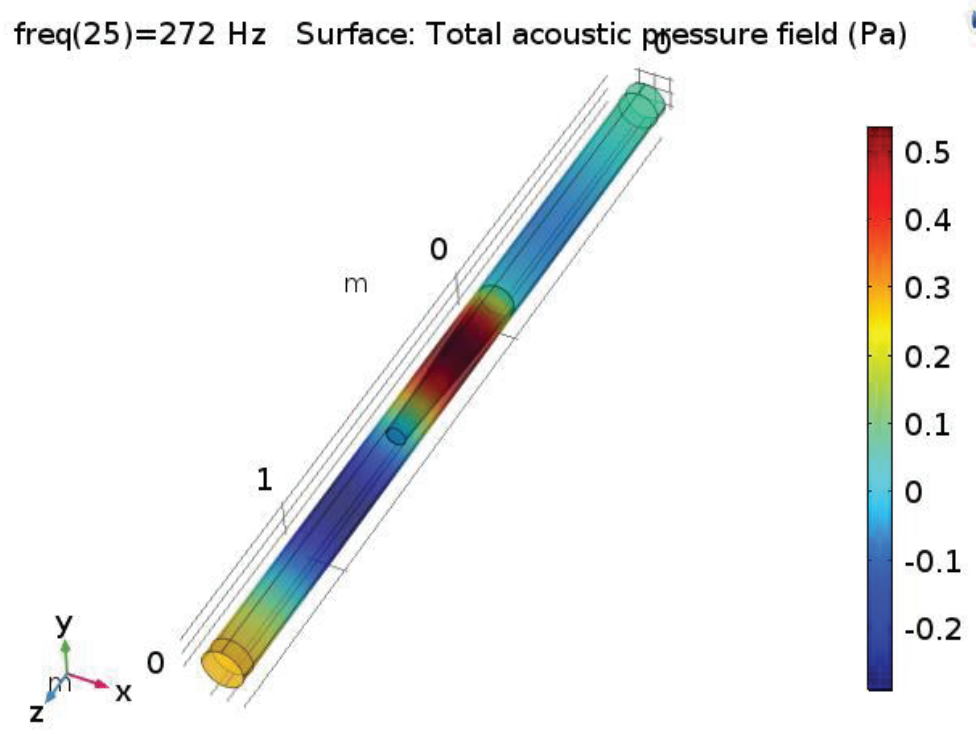

Figure 4.10: Acoustic pressure filed of the funnel resonator at the first eigen-frequency.

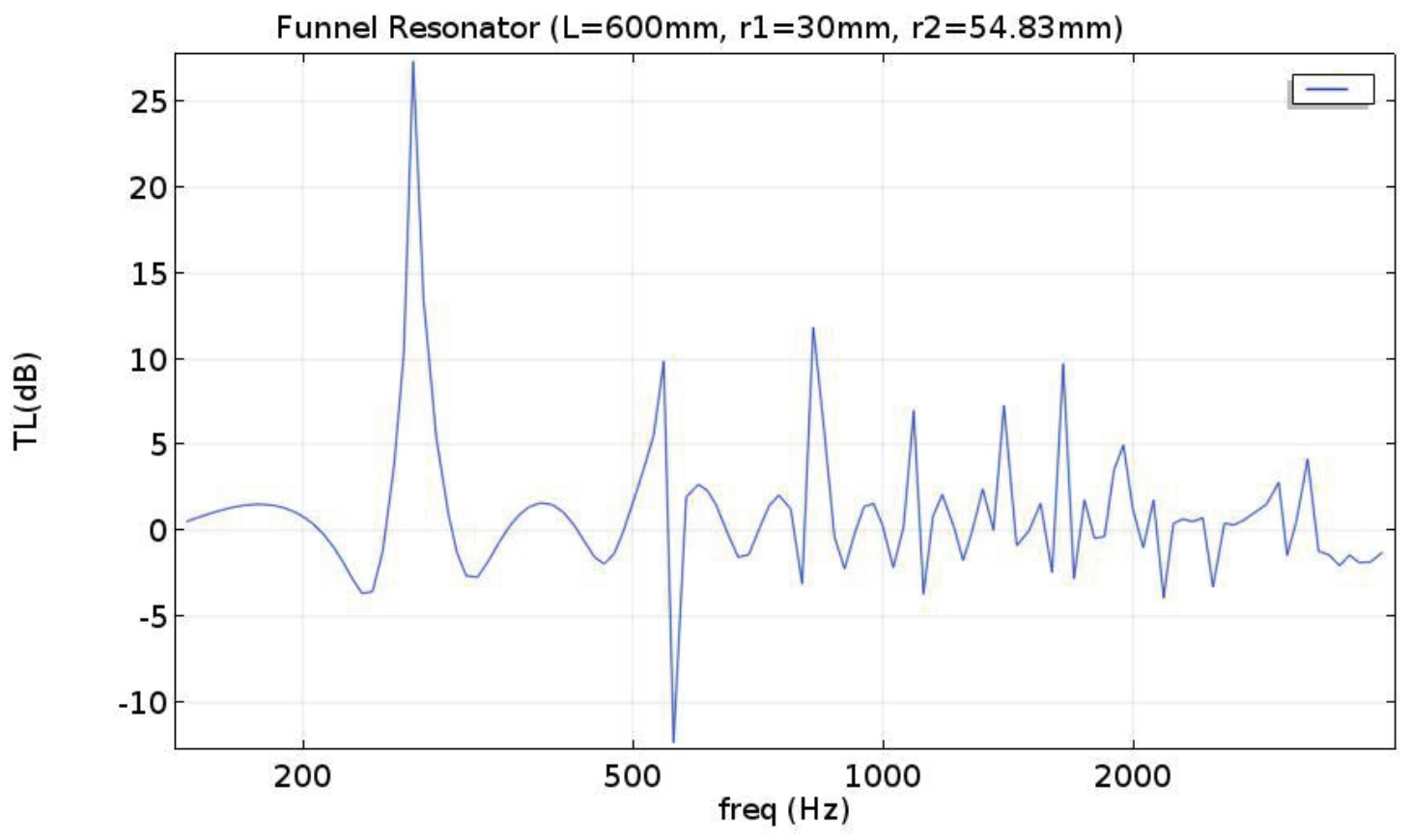

Figure 4.11: Transmission loss of the funnel resonator.

As can be seen in Fig. 4.11, the second and third eigen-frequencies, i.e., $553 \mathrm{~Hz}$ and $831 \mathrm{~Hz}$ are 2 and 3 times of the first one, $273 \mathrm{~Hz}$. 


\subsection{Spiral-Helicoidal Resonator}

The combination of the spiral and helicoidal resonators will give lower eigen-frequencies in comparison with the helicoidal resonator. For instance, the acoustic pressure field of the SHR with $0.6 \mathrm{~m}$ length, 2 spiral turns, and $61^{\circ}$ of helical turning is presented in Fig. 4.12 at its third eigen-frequency.

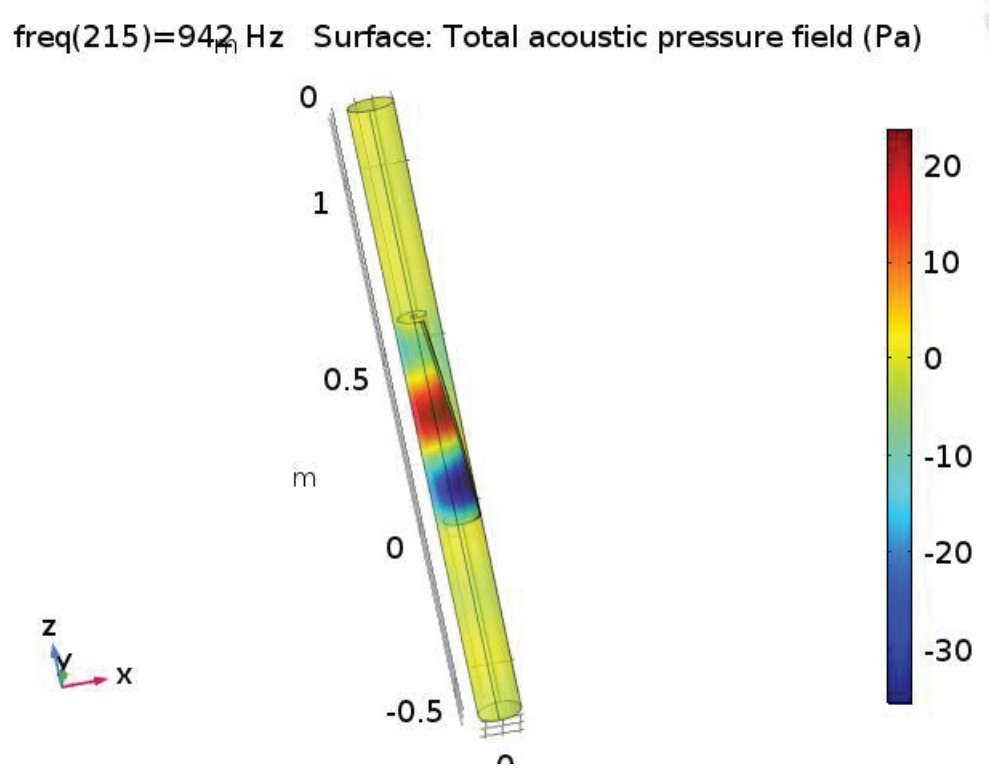

Figure 4.12: Acoustic pressure field of the spiral-helicoidal resonator at the third eigen-frequency.

Moreover, the transmission loss of this SHR is presented in Fig. 4.13:

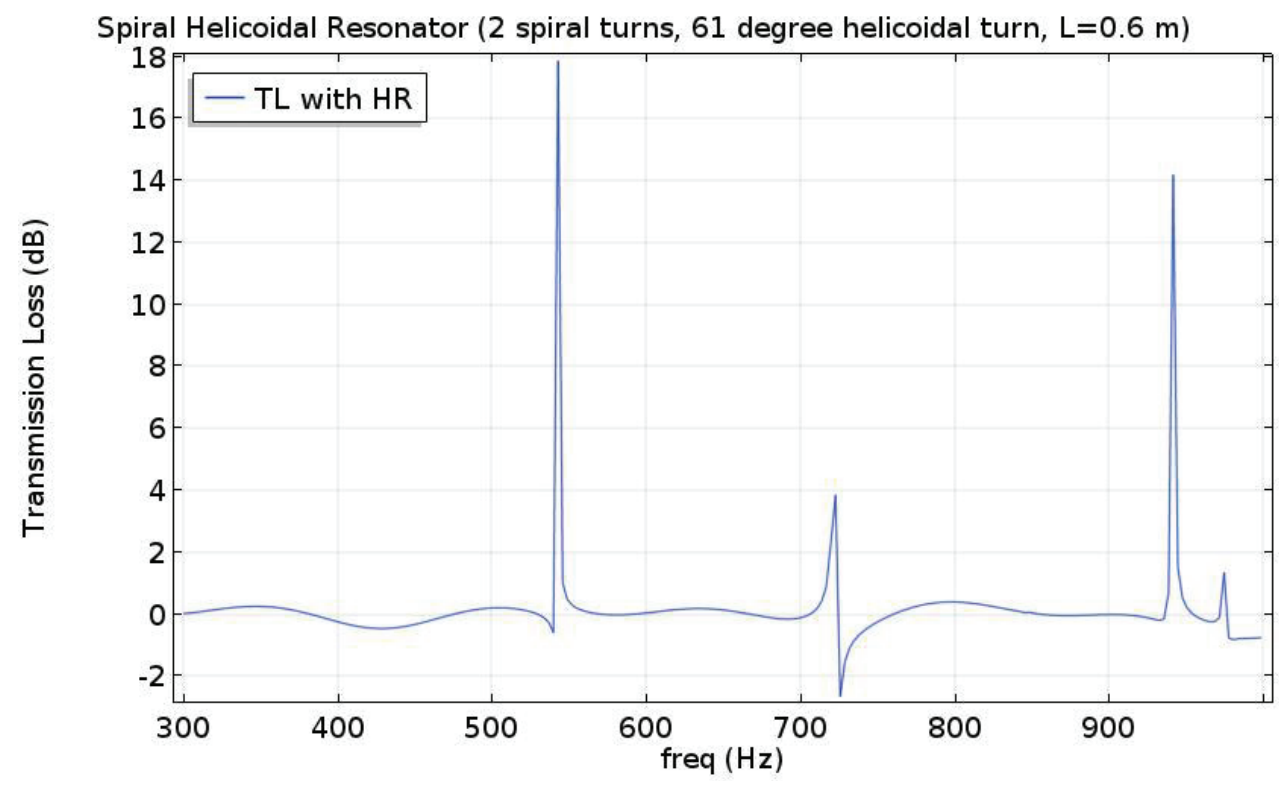

Figure 4.13: Transmission loss of the spiral_helicoidal resonator. 


\subsection{Funnel-Spiral-Helicoidal Resonator}

The combination of these various resonators will give results which are better for broadband noise reduction while the fabrication process will become more complicated. For instance, the TL result of the FSHR is presented in Fig. 4.14 to show that the low frequency noises can be absorbed with only $0.45 \mathrm{~m}$.

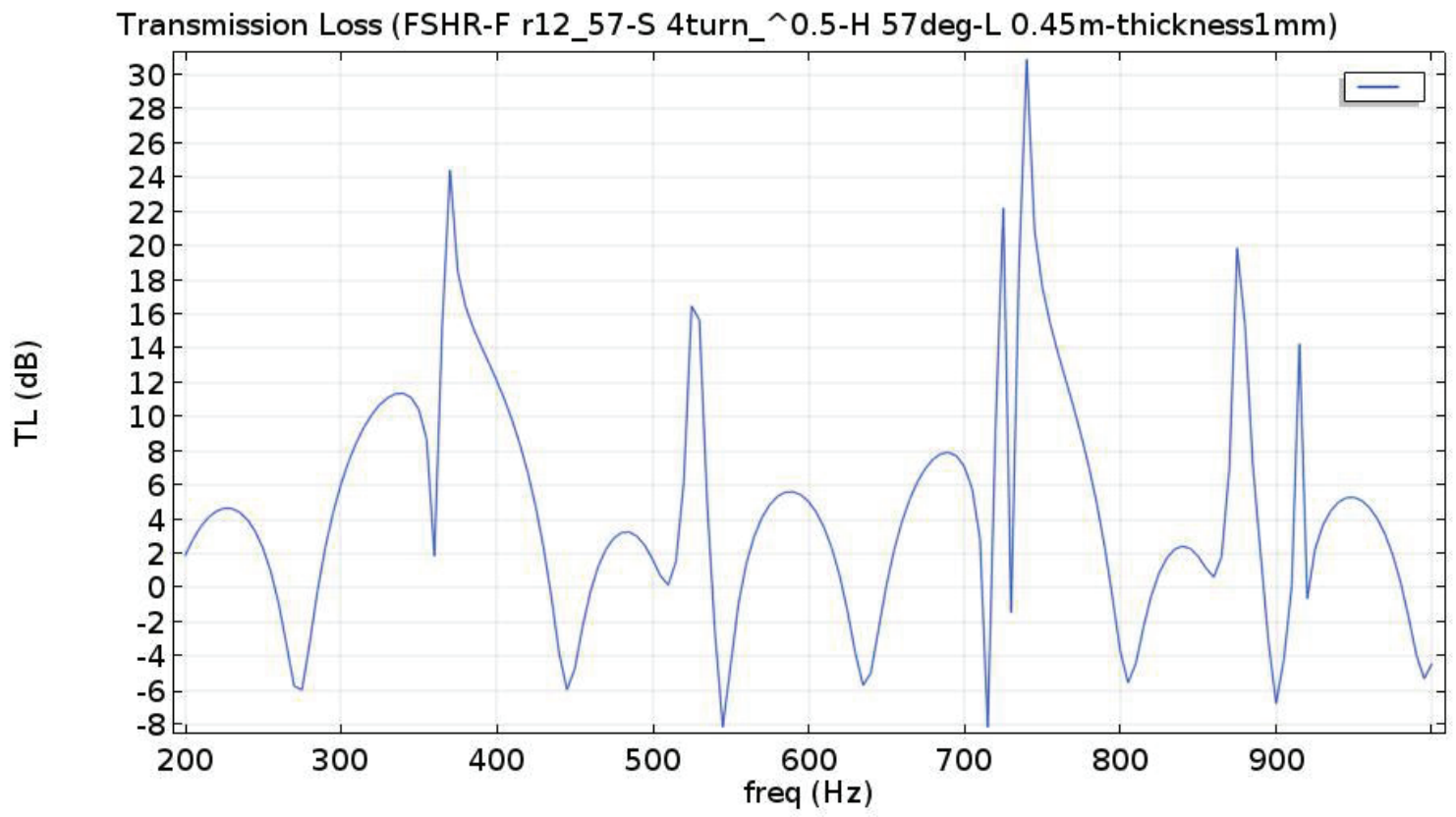

Figure 4.14. Transmission loss of the funnel_spiral_helicoidal resonator with $0.45 \mathrm{~m}$ length.

\subsection{Acoustic Black Hole muffler}

As mentioned in the previous chapter, the Acoustic Black Hole muffler can be installed inside the T-joint. For instance, the acoustic pressure field at $300 \mathrm{~Hz}$ is shown in Fig. 4.15: 


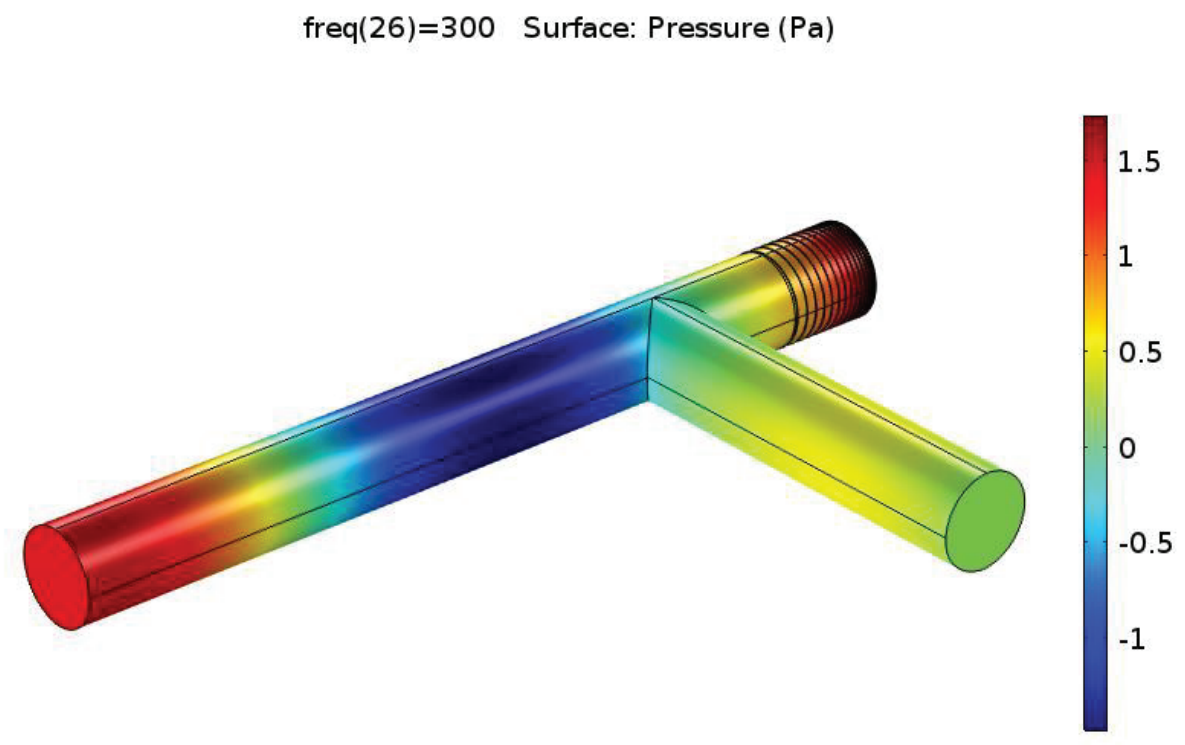

Figure 4.15. Acoustic pressure field of the $\mathrm{ABH}$ at $300 \mathrm{~Hz}$.

This model is simulated similar to the real test setup that it will be discussed in Chapter 6, Section 5. The transmission loss of the ABH muffler with $135 \mathrm{~mm}$ length is shown in Fig. 4.16.

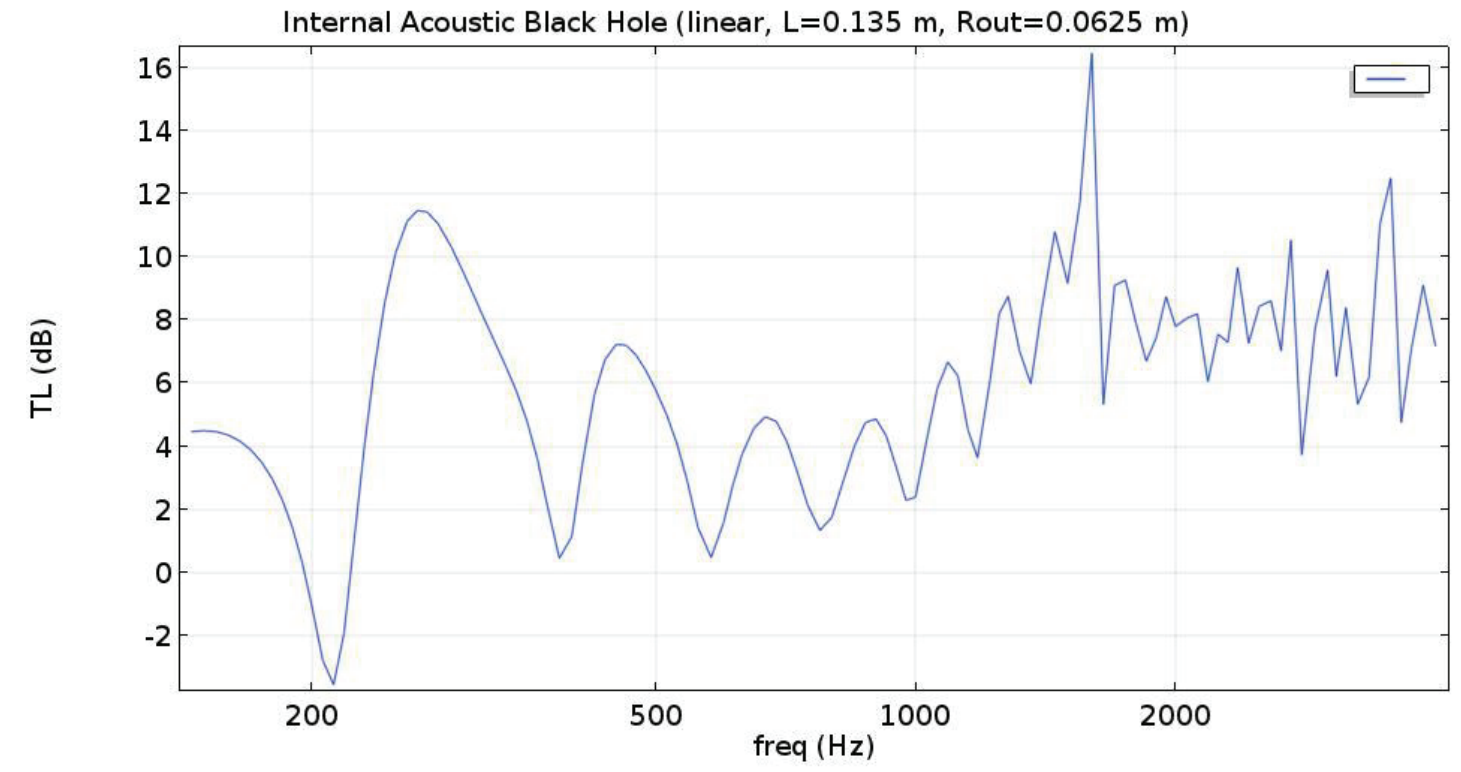

Figure 4.16. Transmission loss of the Acoustic Black Hole muffler.

As can be seen in Fig. 4.16, the ABH muffler can absorb broadband noise. The main noise reduction occurs between $230 \mathrm{~Hz}$ and $1700 \mathrm{~Hz}$. The eigen-frequencies of this model are 285, $433,609,731,834,941,1055,1178,1306,1452,1610,1619,1620,1624,1631,1632 \ldots \mathrm{Hz}$. 


\subsection{External Acoustic Black Hole muffler}

Due to the long channel needed to reduce the low frequency noise by the EABH muffler (see Fig. 3.16), the developed shape of external Acoustic Black Hole muffler should be used to achieve the desired goal in a short length. For instance, the front section view of half part of the EABH muffler is shown in Fig. 4.17 with $182 \mathrm{~mm}$ length, $62.5 \mathrm{~mm}, 127 \mathrm{~mm}$ inner and outer radiuses. The performance of this model is similar to the known External Acoustic Black Hole muffler.

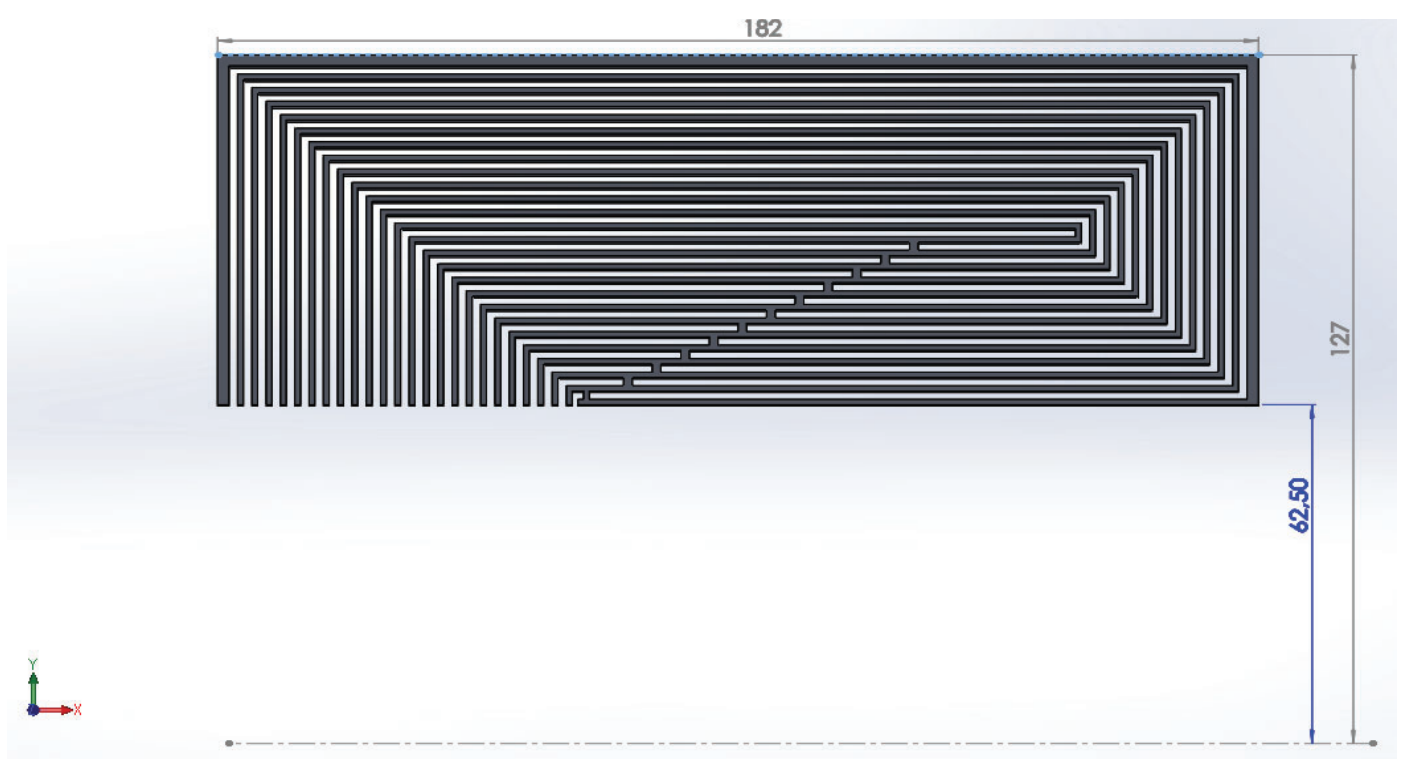

Figure 4.17. Front section view of the external Acoustic Black Hole muffler.

The transmission loss of the mentioned EABH muffler by considering Acoustic-Structure Interaction is depicted in Fig. 4.18: 


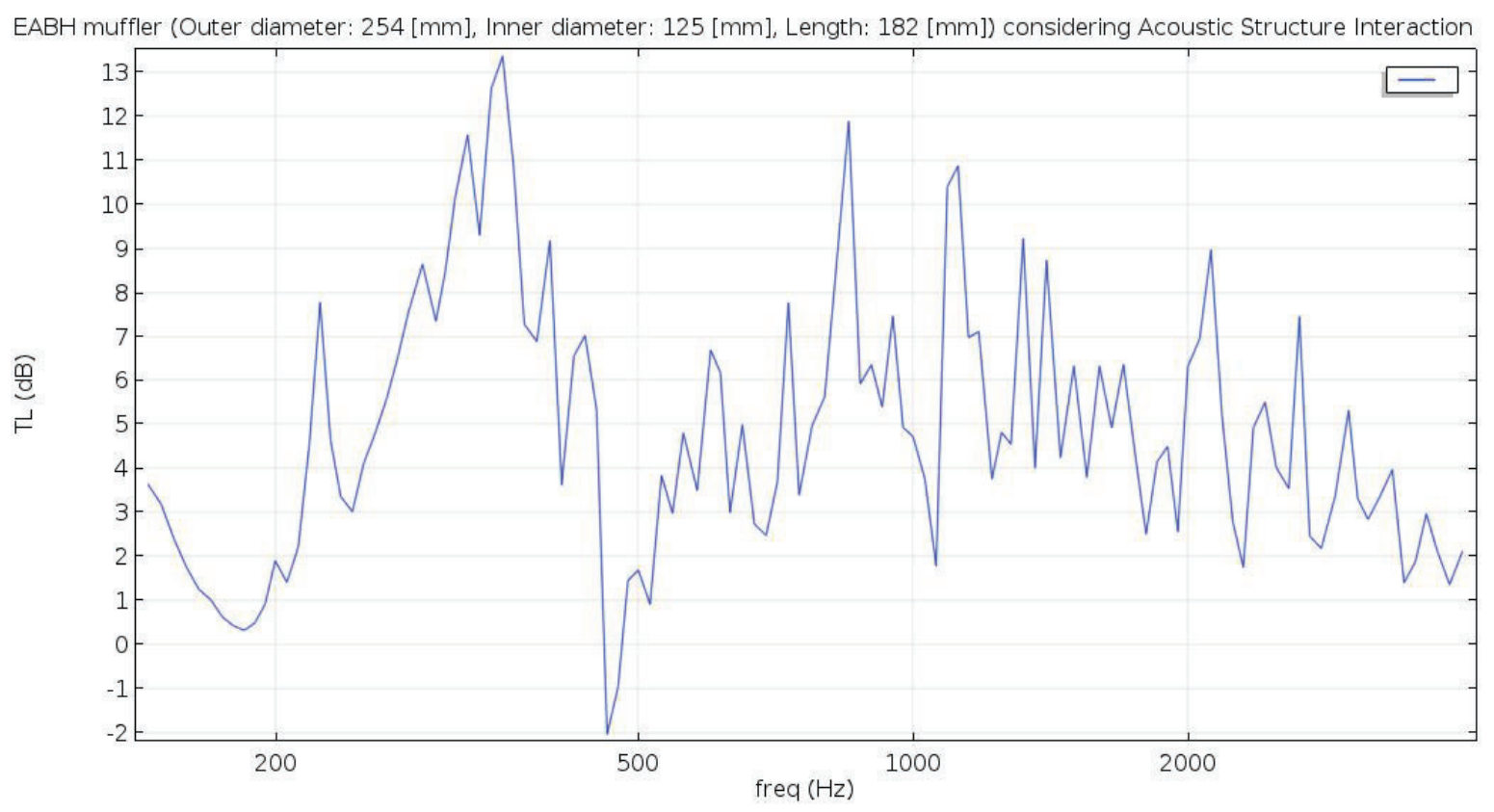

Figure 4.18: Transmission loss of the external Acoustic Black Hole muffler.

As can be seen in Fig. 4.18, this type of the muffler can be used as a broadband noise reduction system which can absorb even the noise at $270 \mathrm{~Hz}$ and even less. But to demonstrate the performance of this muffler, the power level of the fan at $325 \mathrm{~m}^{3} / \mathrm{h}$ flowrate is considered then the EABH muffler is installed surrounding the duct in COMSOL simulation. The Insertion loss by considering Acoustic-Structure Interaction (ASI) is evaluated afterwards. The above EABH muffler could deduct $6.2 \mathrm{~dB}$ shown in Fig. 4.19: 


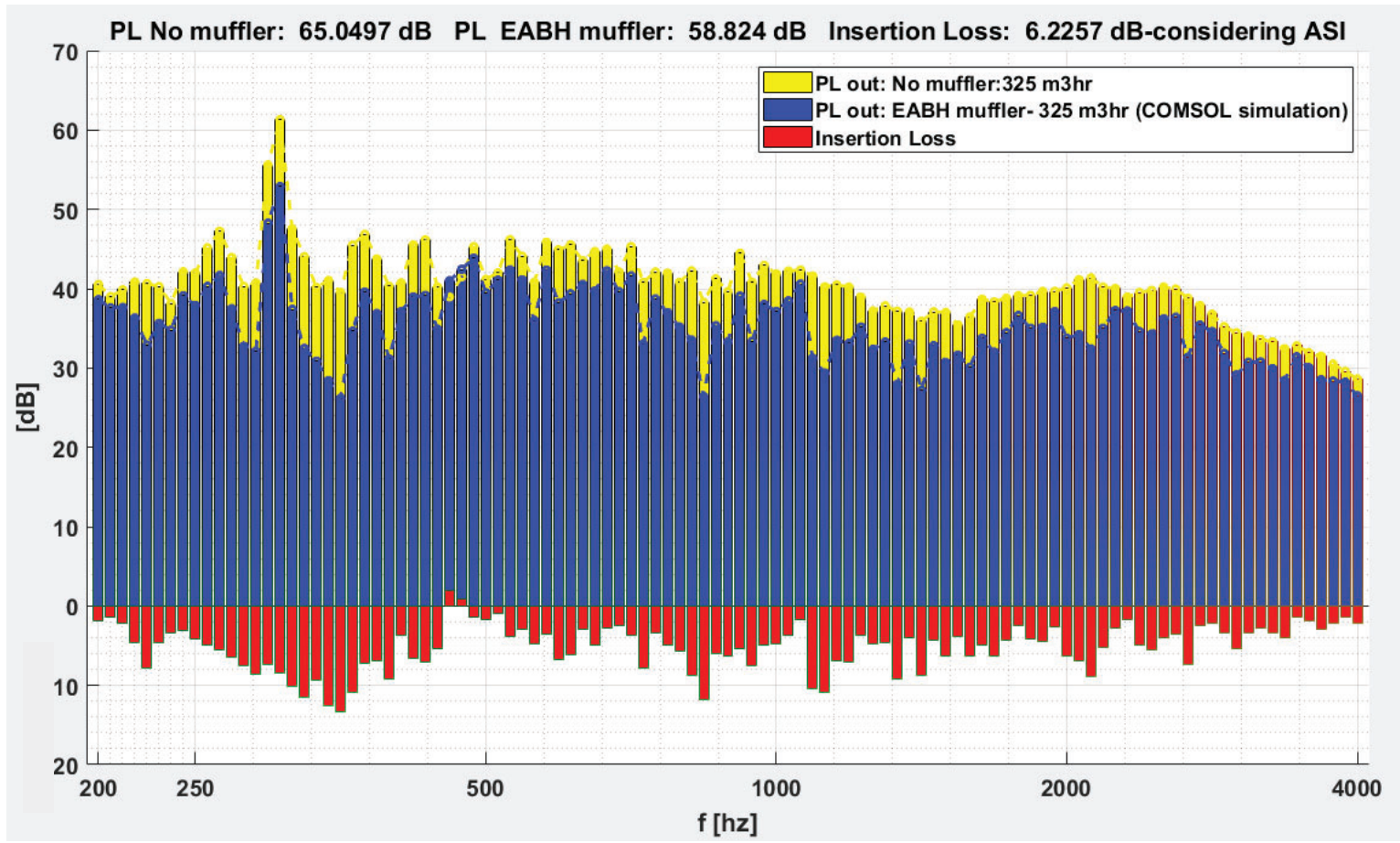

Figure 4.19: power level and Insertion Loss of the EABH muffler with considering ASI

If acoustic structure interaction is not considered, then the results will be like in Fig. 4.20:

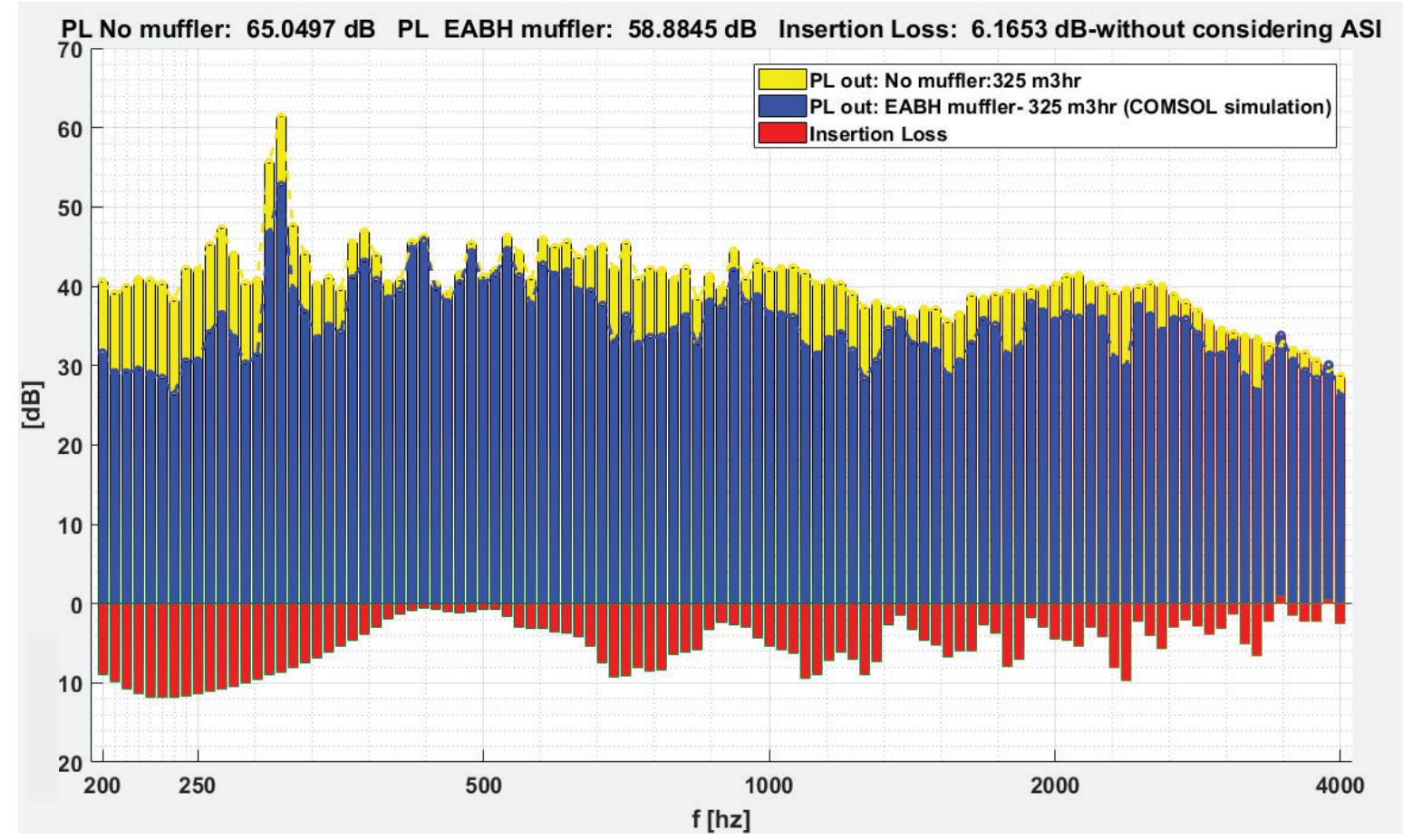

Figure 4.20: Power level and transmission loss of the EABH muffler without considering ASI.

Comparing Fig. 4.19 and Fig. 4.20 reveals that a big differences is observed when ASI is not considered at low frequencies. Hence, it is recommended to use multi-physics module in IABH 
and $\mathrm{EABH}$ simulation. Using three series EABH with $182 \mathrm{~mm}$ length, in total $546 \mathrm{~mm}$, can be one of the best options to deduct the broadband noise more than $18 \mathrm{~dB}$.

\subsection{Cylindrical muffler}

The new innovation termed Cylindrical Muffler (CM) is simulated to evaluate the performance of this muffler. For instance, the front section view of the CM with 25 compacted Loops is shown in Fig. 4.21.

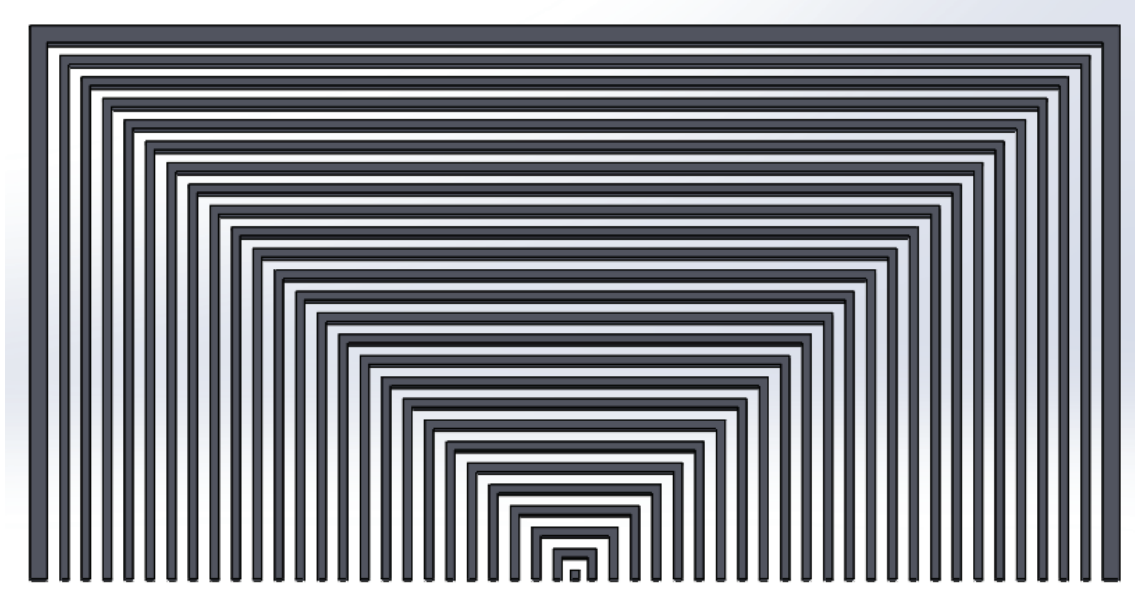

Figure 4.21: Front section view of the cylinder resonator with 25 compact Loops.

Moreover, in Fig. 4.22, the acoustic pressure field of the one loop CM with the outer radius $0.23 \mathrm{~m}$, and $0.33 \mathrm{~m}$ length at the first eigen-frequency $(248 \mathrm{~Hz})$ is demonstrated.

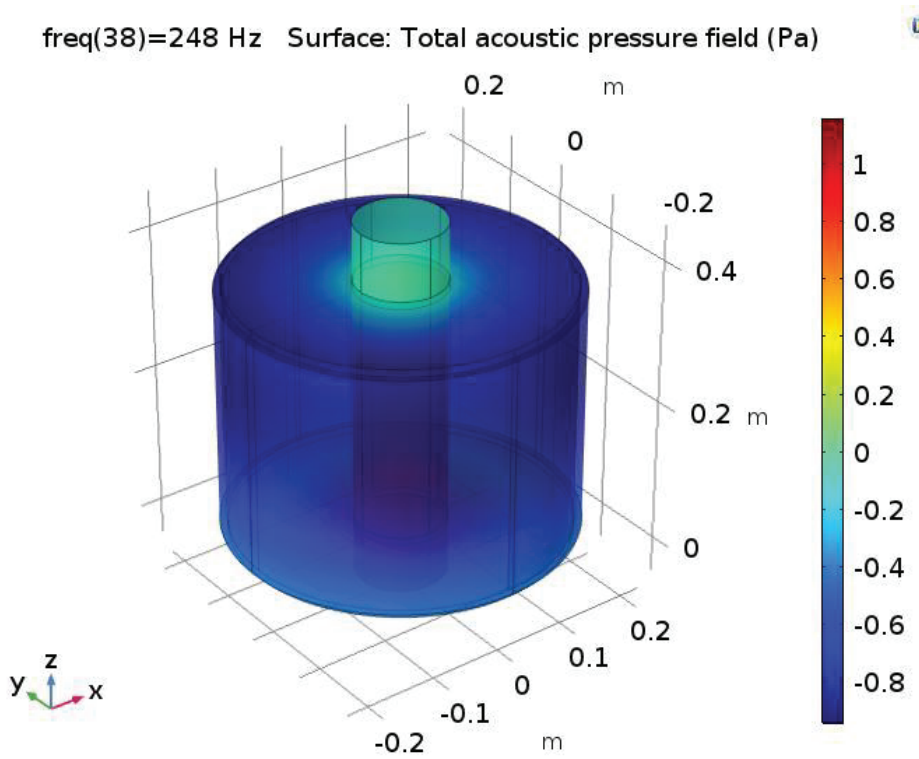

Figure 4.22: Acoustic pressure field of the one loop cylindrical muffler at the first eigen-frequency. 
Transmission loss of the one loop cylindrical muffler is depicted in Fig. 4.23. As can be seen, the second and third eigen-frequencies are 2 and 3 times of the first one which is not possible to achieve with the known side resonators nor Acoustic Black Hole mufflers.

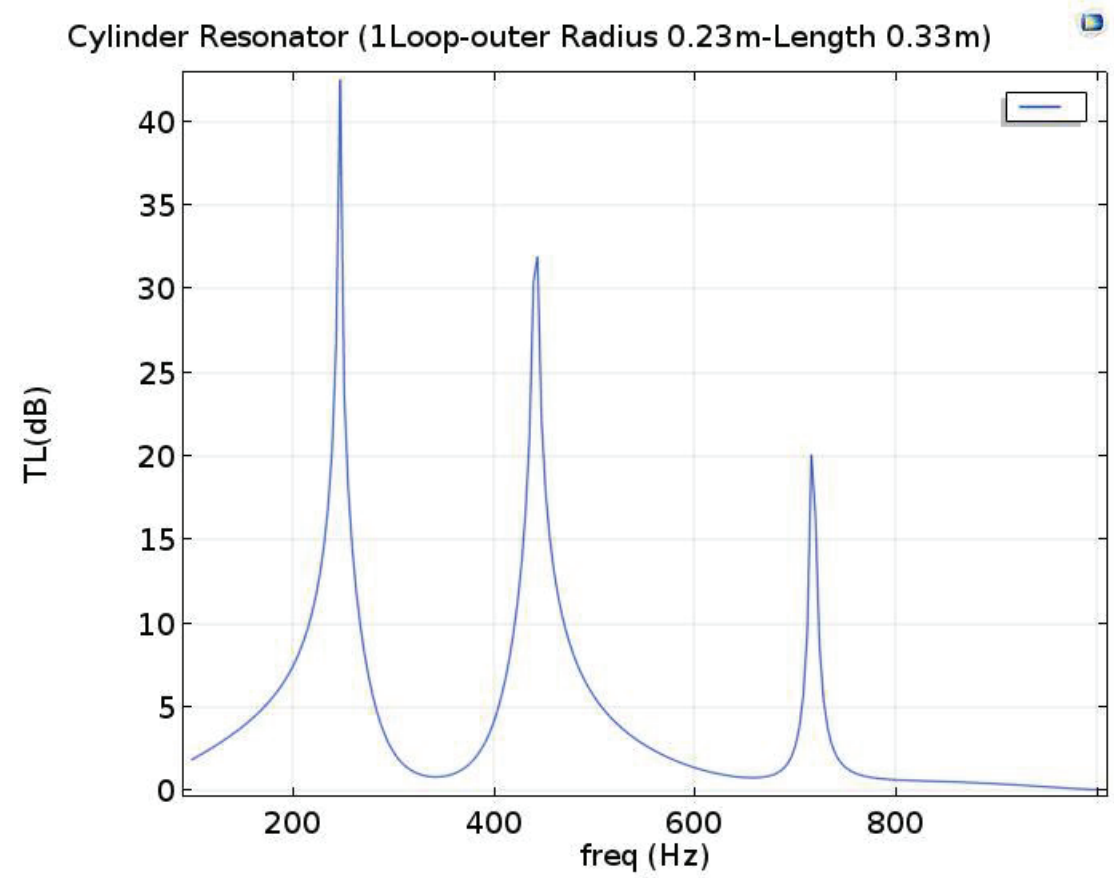

Figure 4.23: Transmission loss of the one loop cylindrical muffler.

Also, the acoustic pressure field of the two loops cylindrical muffler inside each other at the first and second eigen-frequencies are exposed in Fig. 4.24:

freq $(27)=204 \mathrm{~Hz}$ Surface: Total acoustic pressure field $(\mathrm{Pa})$

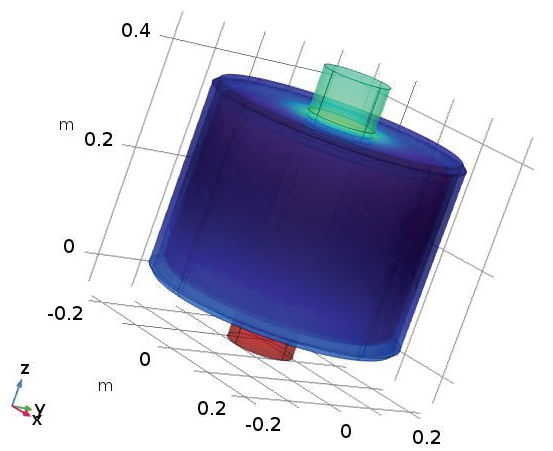

a freq $(82)=424 \mathrm{~Hz}$ Surface: Total acoustic pressure field $(\mathrm{Pa})$ $0.2 \mathrm{~m}$
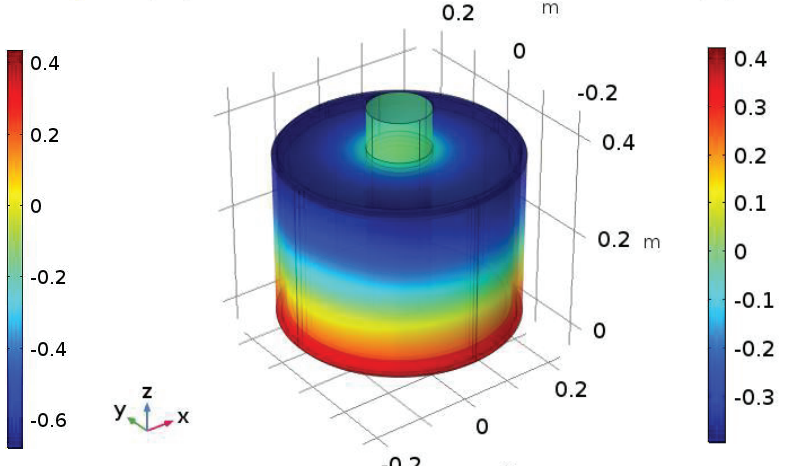

Figure 4.24: Acoustic pressure field of the two loops CM at the first and second eigen-frequencies.

The TL of the CM with 2 loops is presented in Fig. 4.25: 


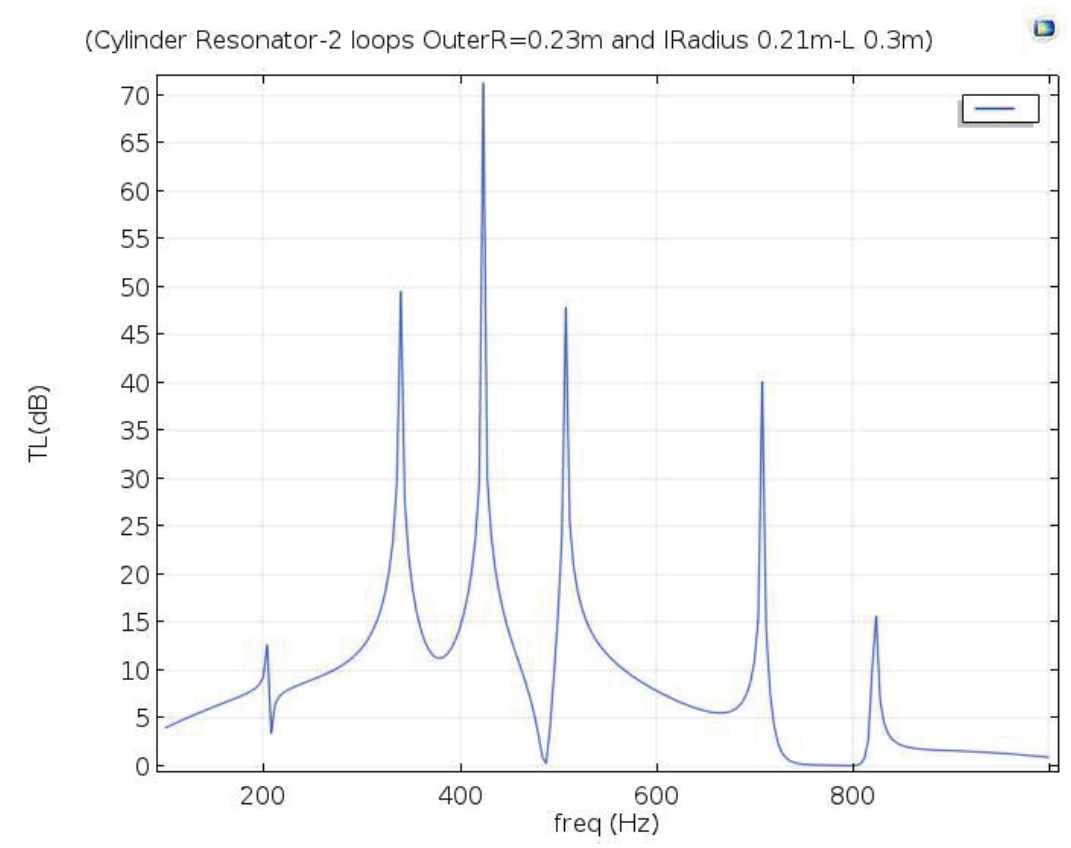

Figure 4.25: Transmission loss of the cylindrical muffler with 2 loops.

Better results could be achieved by increasing the number of loops. For instance, the transmission loss of the mentioned CM with 25 compacted Loops (see Fig. 4.21) is shown in Fig. 4.26. The thickness of the walls is $1 \mathrm{~mm}$ and the distance between walls is $1.5 \mathrm{~mm}$. As can be seen in Fig. 4.26, the cylinder resonator with only $125 \mathrm{~mm}$ length can reduce the noise 9 $d B$. The point is that this resonator is useful for the frequencies more than $1000 \mathrm{~Hz}$.

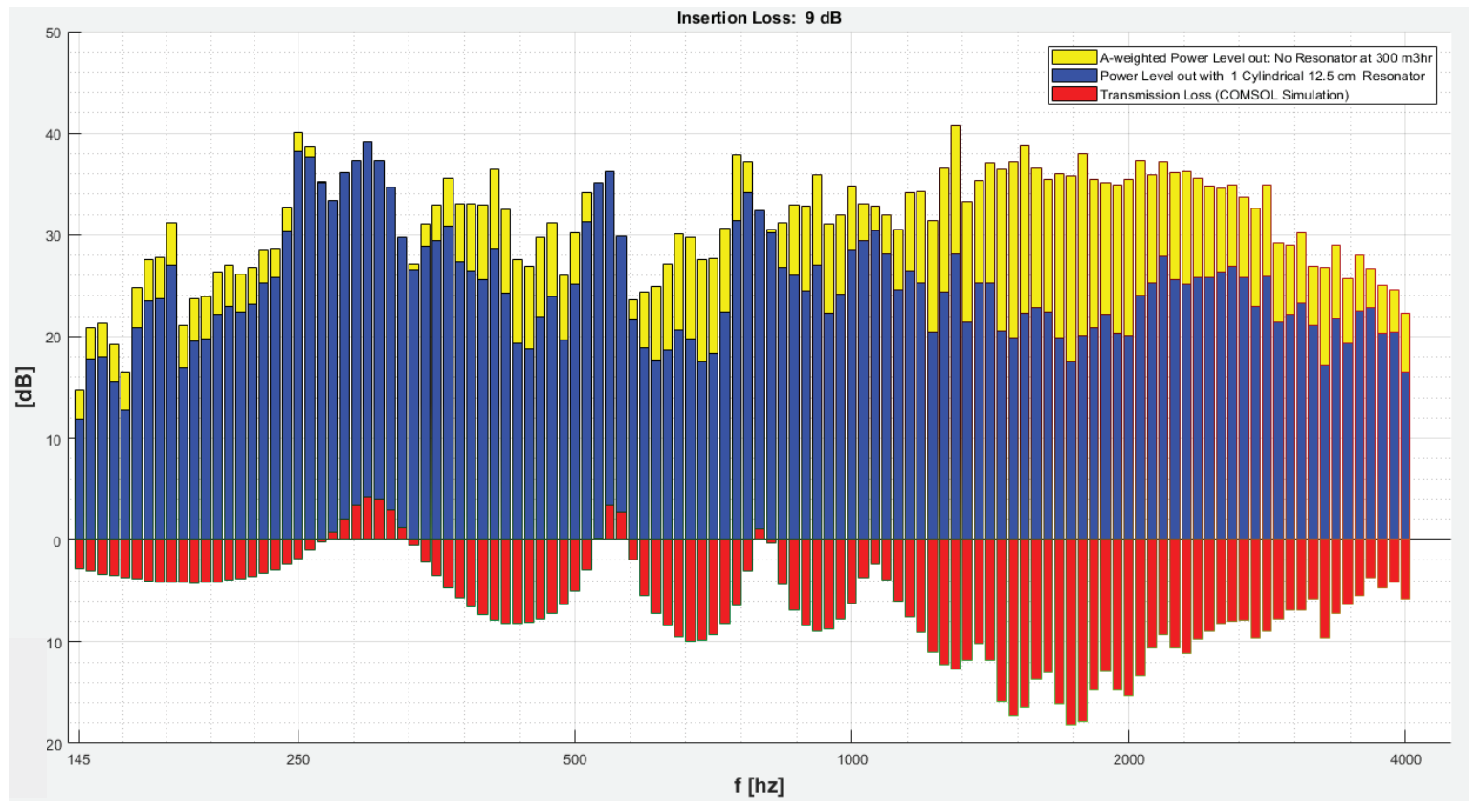

Figure 4.26: Transmission loss of the cylindrical muffler with 25 loops. 
On the other hand by increasing the length of the $\mathrm{CM}$, the low frequency noise can easily be absorbed. For instance, adding three long models of CM shown in Fig. 4.27 with $230 \mathrm{~mm}$ length, in total $690 \mathrm{~mm}$, has a strong ability to remove the noises around $350 \mathrm{~Hz}$. This result is depicted in Fig. 4.28:

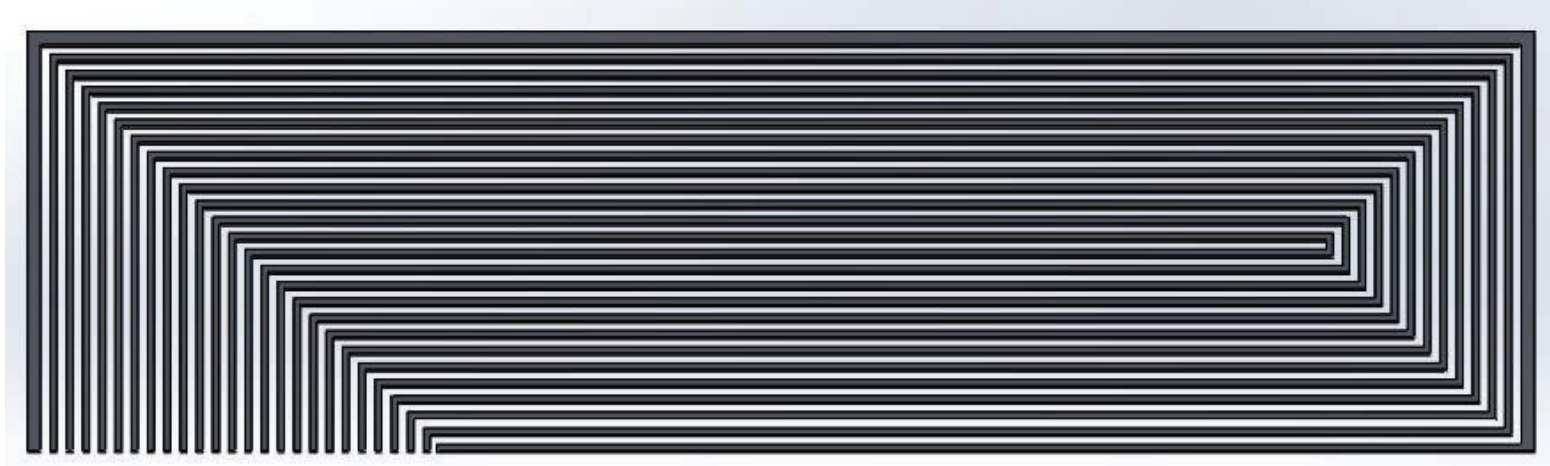

Figure 4.27: Front section view of the long cylinder resonator with 12 Loops.

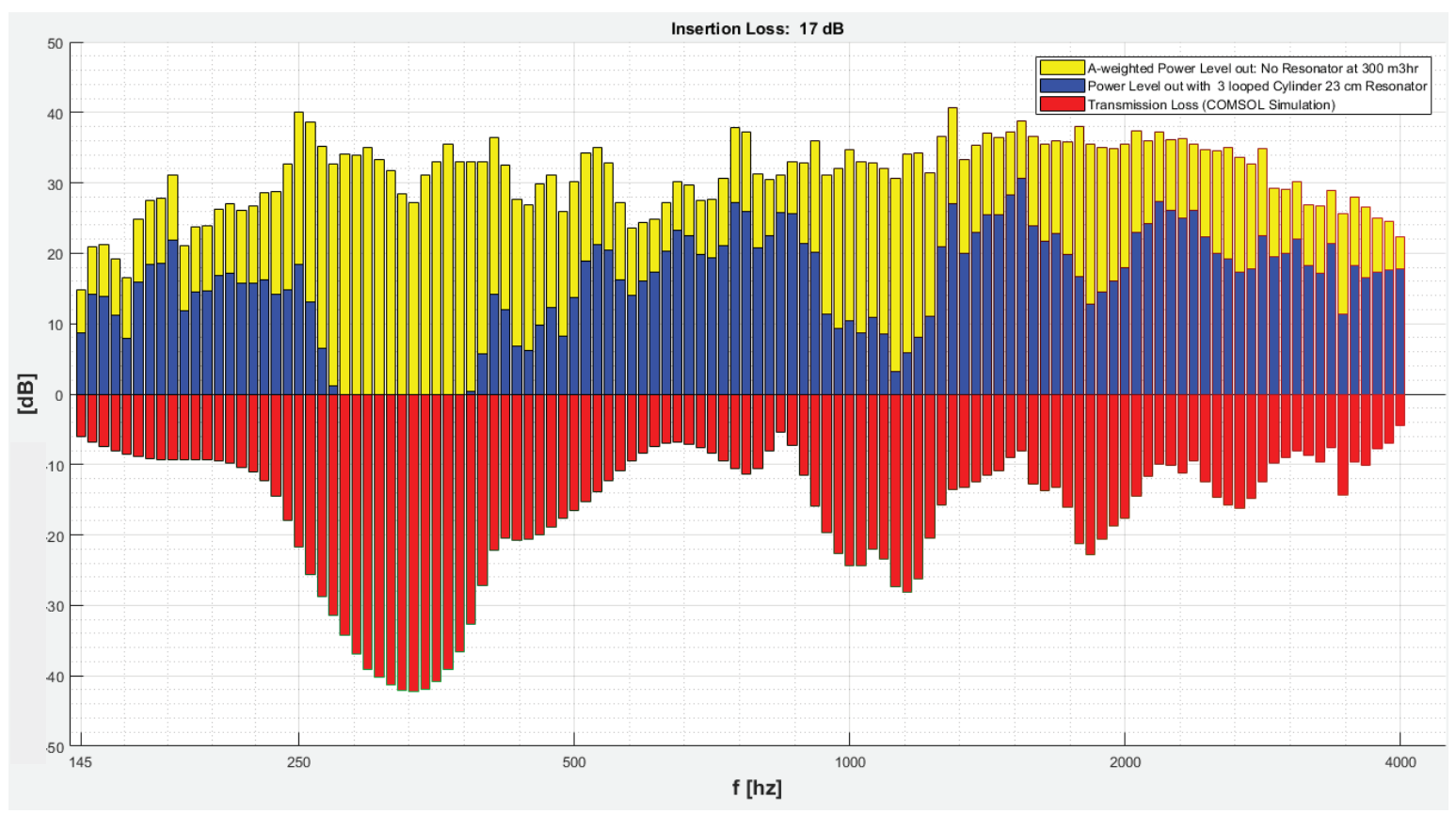

Figure 4.28: Transmission loss of the three long cylindrical muffler $\left(3^{*} 230 \mathrm{~mm}\right)$ with 12 loops.

Based on the simulation result of the EABH and CM between $270 \mathrm{~Hz}$ and $400 \mathrm{~Hz}$ which are shown in Fig. 4.19 and Fig. 4.28, it can be concluded that the combination of the EABH muffler and cylindrical muffler could easily remove noises at any desired frequencies. 


\subsection{Internal Acoustic Black Hole muffler}

The ventilation unit of the Brink Climate Systems is depicted in Fig. 4.29. This unit has two fans which transfer the airflow via two diffusers, shown in Fig 4.29, to the residential space and outside of the house. To hear less noise in residential space, two solutions can be implemented by applying external Acoustic Black Hole muffler. First the muffler can be considered as an accessory and installed outside the unit, second the muffler can be installed inside the unit in the diffuser. The second solution has more priority than the first one for the stakeholders, however it produces more pressure drop which means the fan needs more power to work and energy efficiency will drop a little bit.

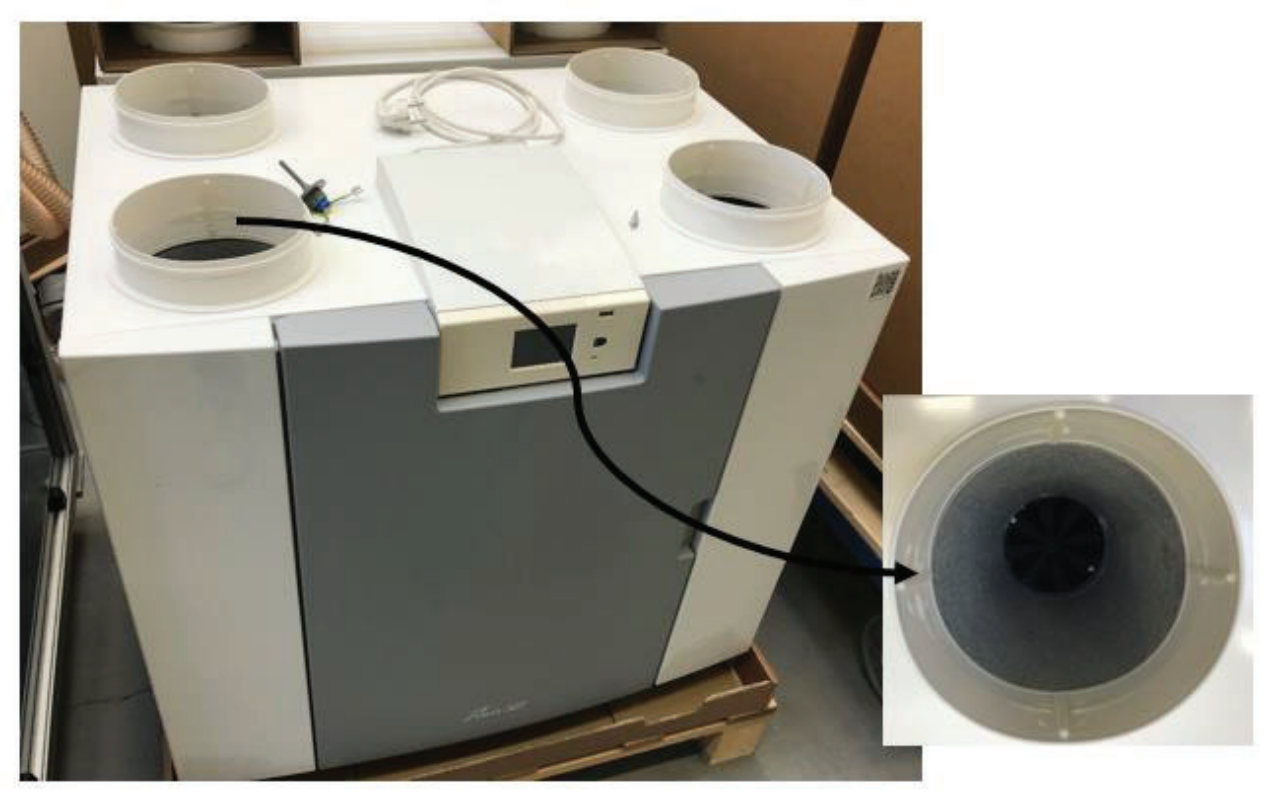

Figure 4.29: Ventilation unit of the Brink Climate Systems.

The object is to design the internal Acoustic Black Hole muffler inside the diffuser. A part of the ventilation unit's model is demonstrated in Fig. 4.30 which involves the muffler inside the diffuser. 


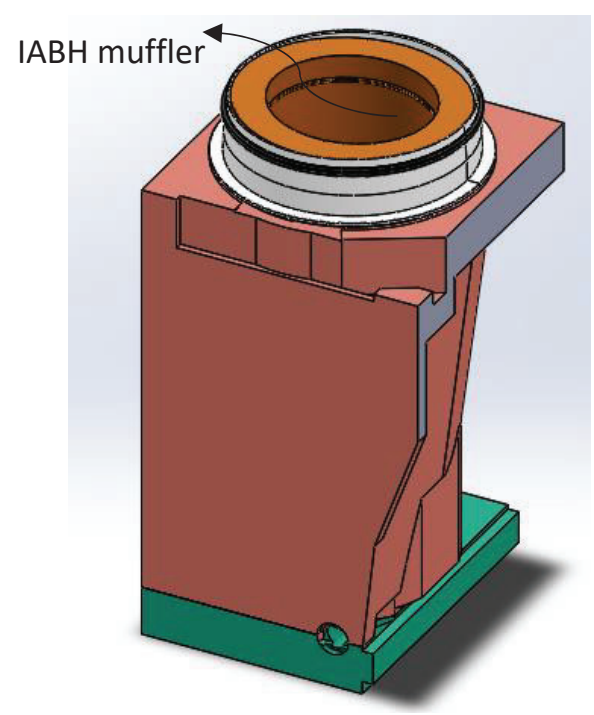

Figure 4.30: The diffuser part of the ventilation unit with the IABH muffler inside it.

As can be seen in Fig. 4.31, the cross section view of the diffuser, due to the unsymmetrical shape of the diffuser the size of the muffler has extra limits.

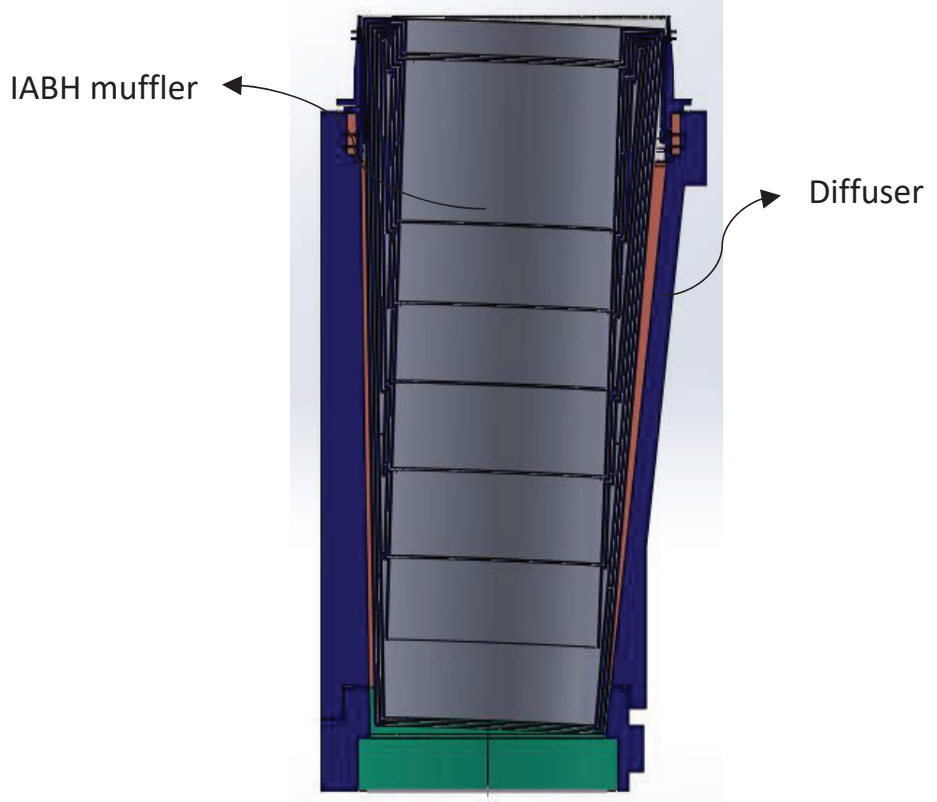

Figure 4.31: The cross section view of the diffuser and the muffler inside the ventilation unit.

The best possible dimension of the diffuser is depicted in Table 4.2. This muffler involves 10 channels to absorb the low frequency noise to high frequency noise based on the fan noise spectrum. To compact the muffler, the channels' width and wall thicknesses are considered 
$1 \mathrm{~mm}$. The inlet of the channels are at the bottom side of the muffler that will be covered by a membrane to protect the muffler from the dust and bacteria growth.

Table 4.2: Dimension of the internal Acoustic Black Hole muffler inside the unit.

\begin{tabular}{|c|c|c|c|}
\hline & \multicolumn{2}{|c|}{ Internal Acoustic Black Hole muffler } & $1 \mathrm{~mm}$ \\
\hline Length & $335 \mathrm{~mm}$ & Blade thickness & 10 \\
\hline Outer diameter & $118-152 \mathrm{~mm}$ & No. of channels & 10 \\
\hline Inner diameter & $108 \mathrm{~mm}$ & & \\
\hline
\end{tabular}

To see the details of the muffler, the front section view of the muffler is plotted in Fig. 4.32.

As can be seen in Fig. 4.32, the closed ends are demonstrated by black circles.

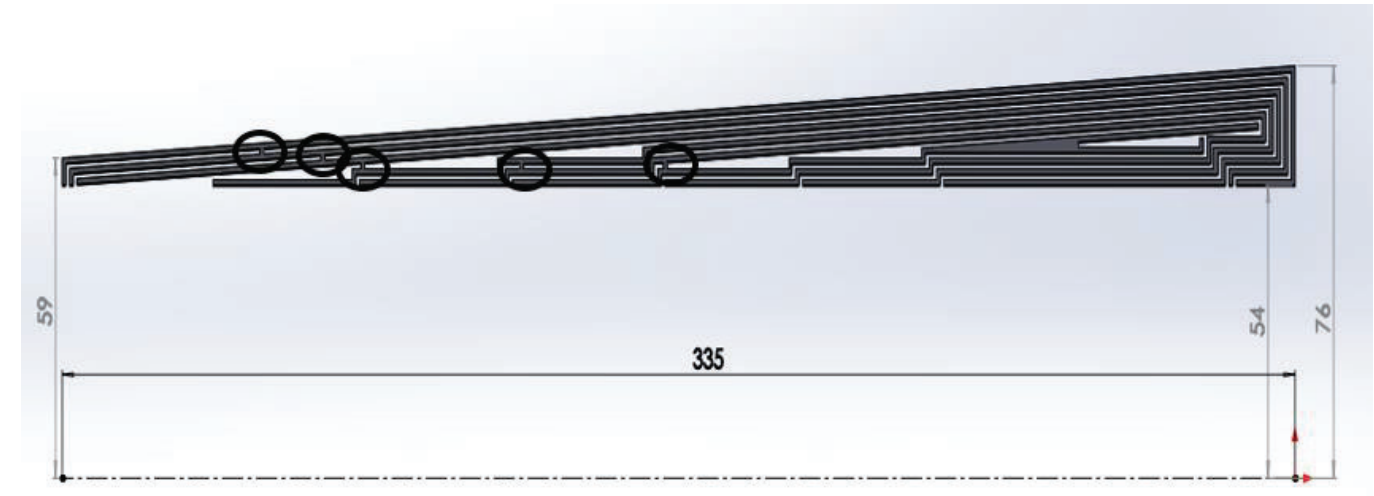

Figure 4.32: The front section view of the IABH muffler inside the ventilation unit.

To make this type of the muffler, one of the solutions is to divide the muffler into some segments like in Fig. 4.33.

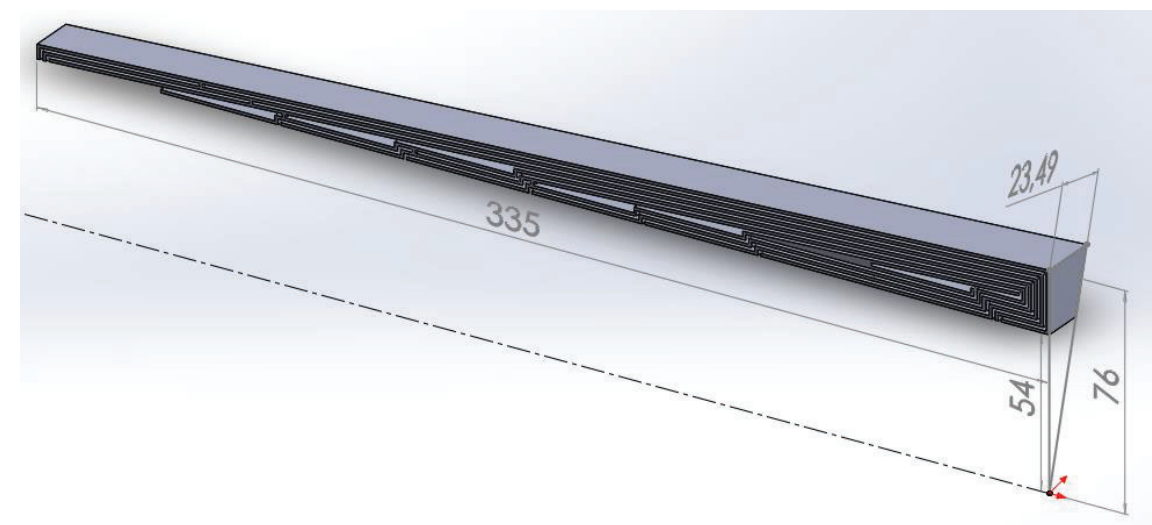

Figure 4.33: The segment of the internal Acoustic Black Hole muffler inside the Ventilation unit.

Assemble twenty pieces of this segment, will give the muffler shown in Fig. 4.34: 


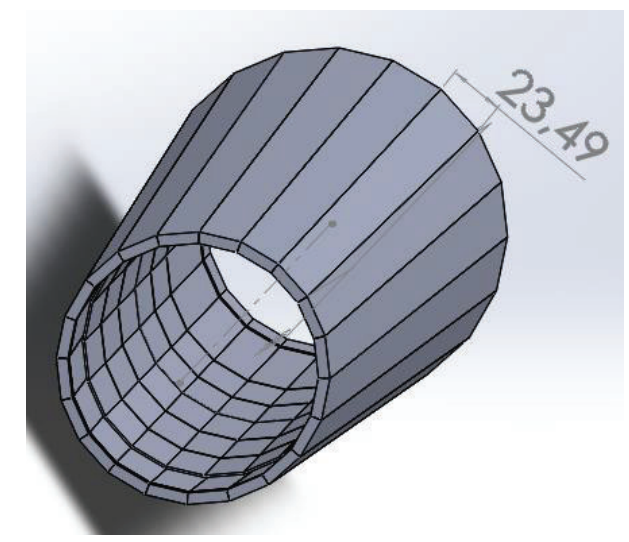

Figure 4.34: The assembly of segments of the internal Acoustic Black Hole muffler inside the unit.

Next, the model should be simulated by COMSOL software to find the acoustic performance of the muffler. Hence, the mesh model of the system is demonstrated in Fig 4.35.

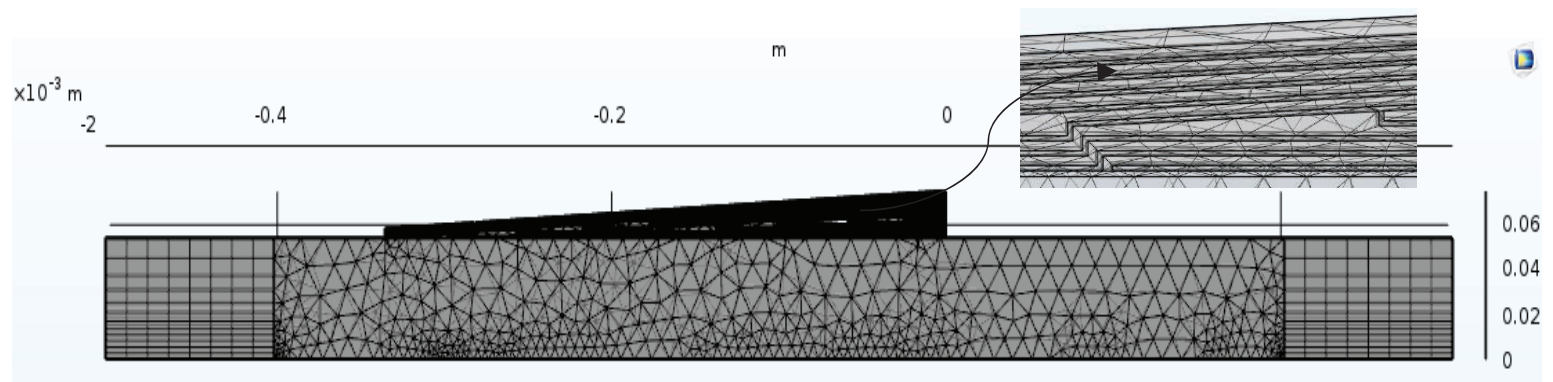

Figure 4.35: The mesh of the internal Acoustic Black Hole muffler model inside the unit.

The maximum length of the mesh is calculated based on considering at least 6 points for the maximum desired frequency which in this case is $4000 \mathrm{~Hz}$ as $\frac{343[\mathrm{~m} / \mathrm{s}]}{4000[1 / \mathrm{s}]^{*} 6}=0.0143[\mathrm{~m}]$.

The transmission loss of the IABH muffler inside the unit is shown in Fig. 4.36. 


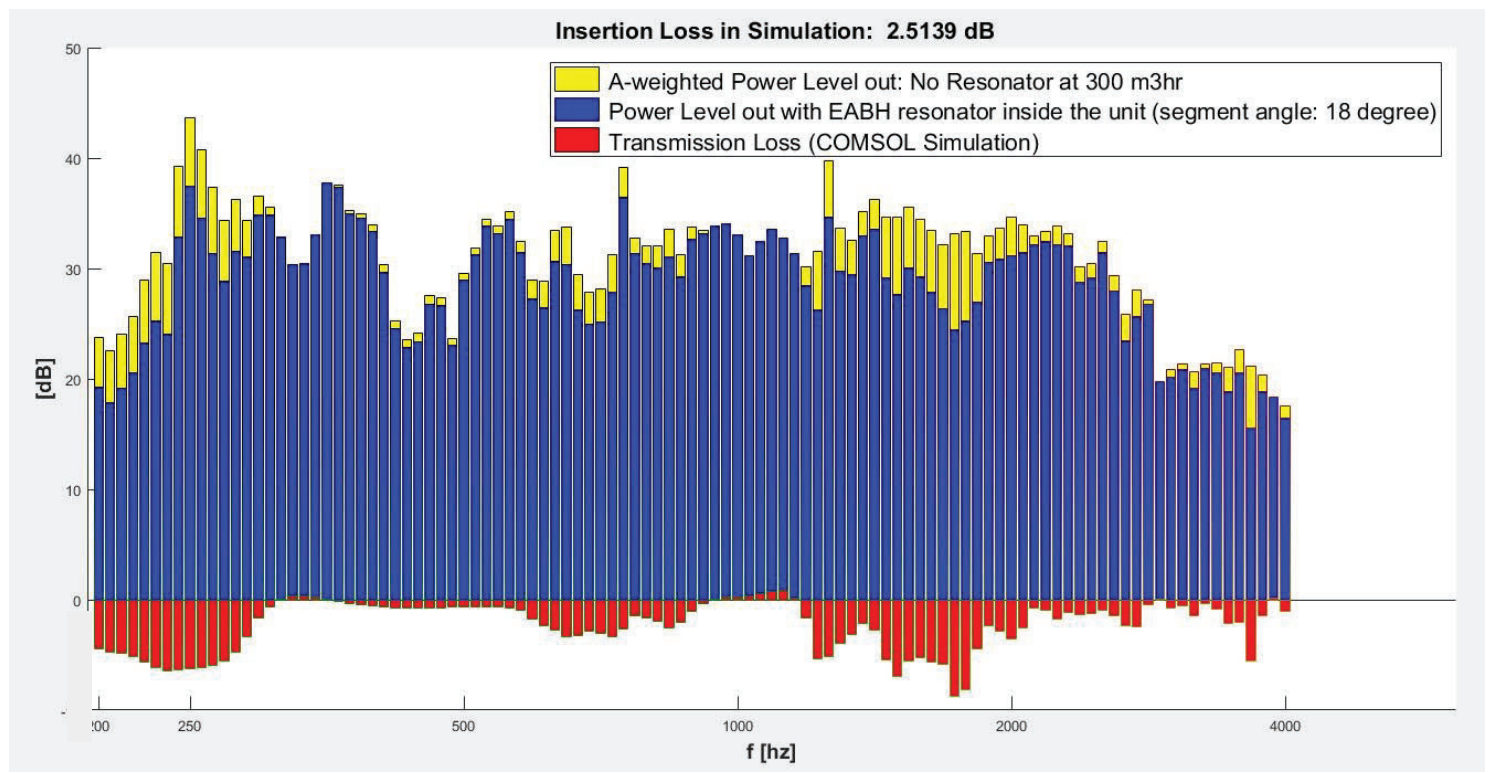

Figure 4.36: Transmission loss of the internal Acoustic Black Hole muffler inside the unit.

It should be noted that if the acoustic structure interaction is considered at the simulation, then the TL will become like in Fig. 4.37. In this model, polyamide is considered as a material of the muffler.

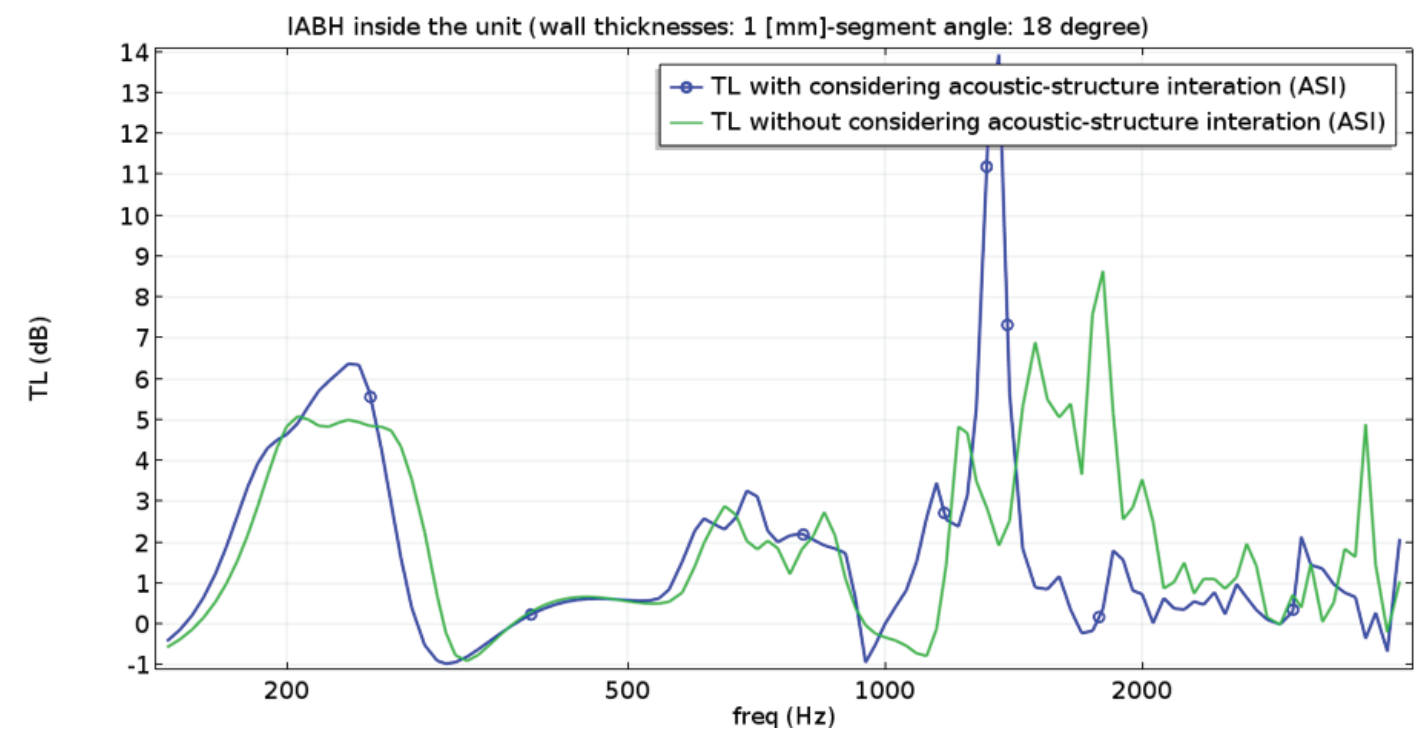

Figure 4.37: TL of the internal Acoustic Black Hole muffler inside the unit by considering ASI.

To present the differences of applying the ASI, the TL of the muffler with four various materials i.e. ABS, PP, and P.A. are compared with the TL of the model with rigid walls in Fig. 4.38: 


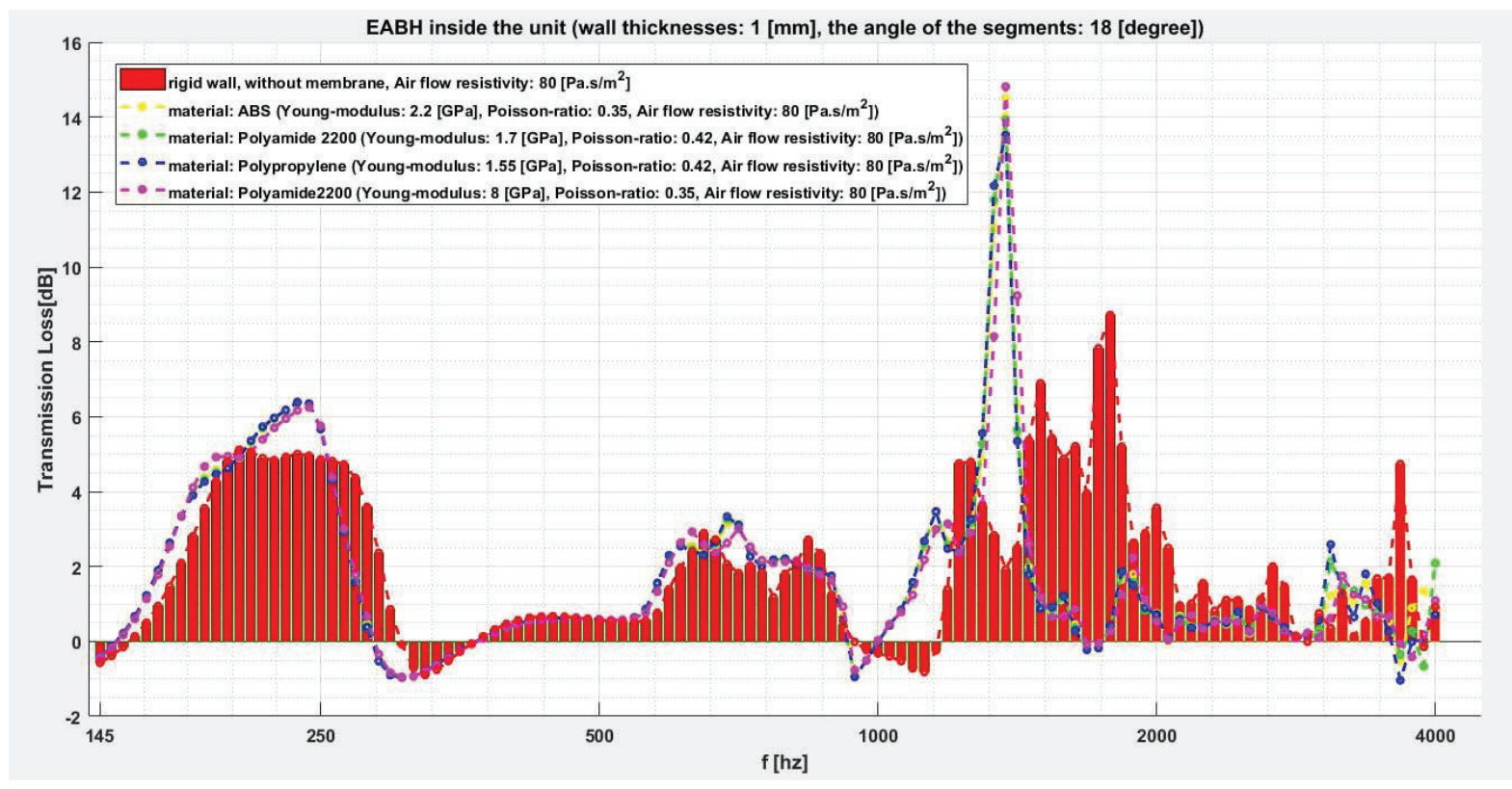

Figure 4.38: TL of the internal Acoustic Black Hole muffler inside the unit.

Moreover, eigen-frequency analyses of the muffler is simulated to determine the first 15 eigen-frequencies for the each state and the results are written in Table. 4.3:

Table 4.3: First to 15 eigen frequencies of the IABH muffler inside the unit.

\begin{tabular}{|c|c|c|c|c|c|c|c|}
\hline \multirow[b]{2}{*}{ No. Material } & \multirow{2}{*}{\begin{tabular}{l} 
Pressure \\
Acoustic \\
module \\
\multicolumn{1}{c}{ air }
\end{tabular}} & \multicolumn{3}{|c|}{$\begin{array}{l}\text { Structure module (with } \\
\text { considering a PVC } \\
\text { membrane) }\end{array}$} & \multicolumn{3}{|c|}{$\begin{array}{l}\text { Acoustic-structure Interaction } \\
\text { (with considering a PVC } \\
\text { membrane) }\end{array}$} \\
\hline & & ABS & PP & PA2200 & $A B S$ & PP & PA2200 \\
\hline 1 & 111 & 2090 & 1883 & 1940 & 182 & 180 & 180 \\
\hline 2 & 139 & 2223 & 2090 & 2090 & 182 & 181 & 181 \\
\hline 3 & 179 & 3375 & 2850 & 2936 & 188 & 187 & 187 \\
\hline 4 & 203 & 3527 & 3017 & 3109 & 190 & 189 & 190 \\
\hline 5 & 207 & 3686 & 3146 & 3241 & 211 & 209 & 210 \\
\hline 6 & 238 & 3818 & 3273 & 3372 & 212 & 210 & 211 \\
\hline 7 & 271 & 3915 & 3376 & 3478 & 232 & 232 & 232 \\
\hline 8 & 289 & 3941 & 3490 & 3596 & 237 & 234 & 235 \\
\hline 9 & 295 & 4107 & 3518 & 3624 & 246 & 243 & 245 \\
\hline 10 & 399 & 4181 & 3725 & 3838 & 248 & 246 & 247 \\
\hline 11 & 498 & 4202 & 3750 & 3863 & 375 & 374 & 374 \\
\hline 12 & 528 & 4357 & 3949 & 4068 & 375 & 375 & 375 \\
\hline 13 & 586 & 4412 & 3955 & 4074 & 381 & 380 & 381 \\
\hline 14 & 605 & 4446 & 3984 & 4104 & 383 & 382 & 382 \\
\hline 15 & 609 & 4482 & 4004 & 4125 & 395 & 394 & 394 \\
\hline
\end{tabular}


To sum up, various resonators and mufflers have been simulated in this chapter to validate the knowledge based designs. It can be concluded that it is possible to absorb low frequency noise via resonators and mufflers. Resonators can work in a specific range of the frequency which is useful for a narrow band treatments (see Fig. 3.2, Fig. 3.6, and Fig. 3.11) and the combination of resonators expand the effective frequency range (Fig. 3.13 and Fig. 3.14). However, mufflers can be considered as a broad band treatments (see Fig. 3.16, Fig. 3.18, Fig. 3.26, and Fig. 3.28). Moreover, mufflers simulations show that the combination of the $E A B H$, $\mathrm{IABH}$, and cylindrical mufflers can remove noises at any desired frequencies. The frequency noise reduction of the helicoidal resonator depends on the diameter of the duct, i.e. for the $125 \mathrm{~mm}$ duct the helicoidal resonator will effect on the frequencies more than $1100 \mathrm{~Hz}$. On the other hand, spiral and funnel resonators can work in any range of the frequencies and it depends on the length and the other specification of the resonators.

Comparing the eigen-frequency of the system with and without considering acousticstructure interaction reveals that mufflers' results are much more related to the ASI rather than resonators. Also, The result of the Fig. 4.38 and Table. 4.3 express that the differences of the eigen-frequencies with various materials are negligible however, it has a big difference with the rigid wall assumption.

Another advantage of the simulation phase is that system's optimizations is occurred. The results of this optimization are used in the next chapter to design details and develop each treatment. 


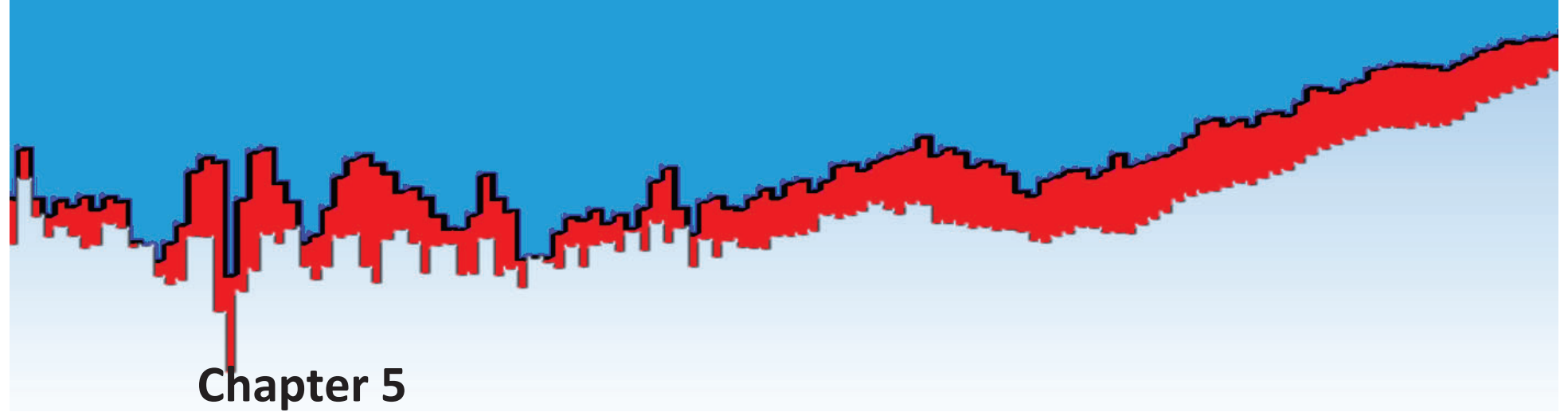

\section{Detail design and development}

\subsection{Introduction}

The performance of the numerical results lead to the development of the first sample. This will result to the detail design and development phase that prototypes are built and tested in the acoustic lab and the reverberation chamber to analyze the technical performance parameters such as the sound power level $(\mathrm{PL})$ and the transmission loss. If $T L$ is more than 3 $\mathrm{dB}$, it means that the method is useful but it may need some optimization to reach the required values. Moreover, if TL is less than $10 \mathrm{~dB}$, then a combination of the second method with the first will be attempted, and the tests will be performed again until it becomes more than $10 \mathrm{~dB}$. The described procedure is demonstrated in Fig. 5.1 and it is presented in the following chapters 5 and 6 .

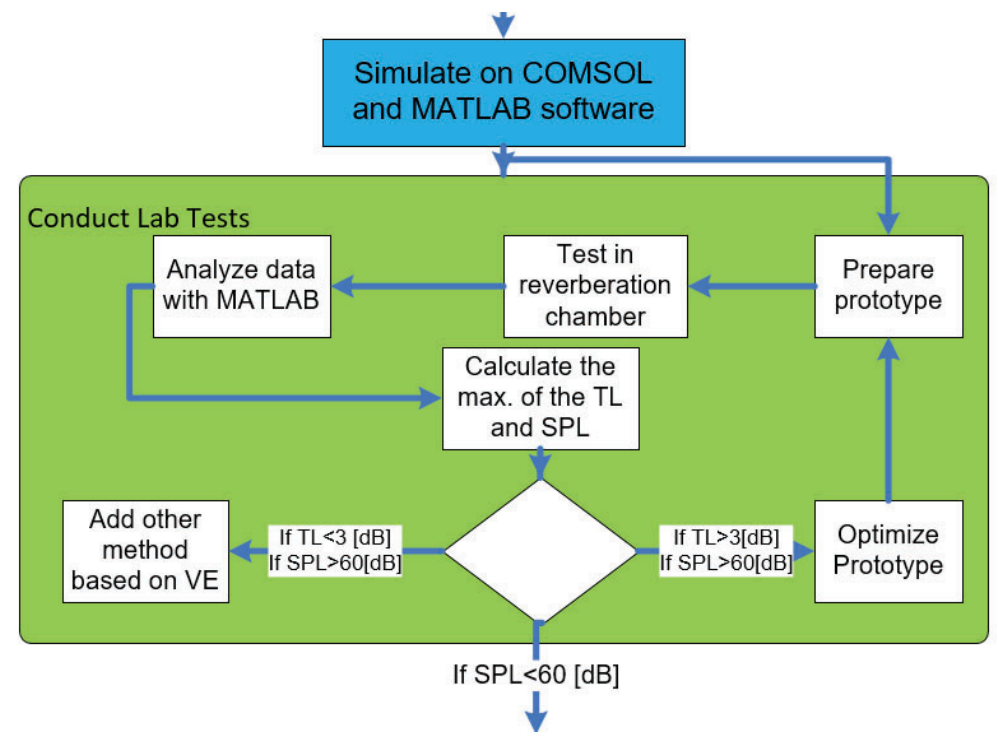

Figure 5.1: Procedure of the Detail Design and Development phase. 


\section{2 Helicoidal resonator}

The nominal diameter of the duct of the ventilation system is $125 \mathrm{~mm}$ and the desired length of the resonator in this project is less than $35 \mathrm{~cm}$. Hence, the helicoidal resonator with the specification in Table 5.1 is designed in SolidWorks software which is shown in Fig. 5.2:

Table 5.1: Specification of the helicoidal resonator.

\begin{tabular}{|c|c|c|c|}
\hline \multicolumn{4}{|c|}{ Helicoidal resonator } \\
\hline Length & $250 \mathrm{~mm}$ & Blade thickness & $5 \mathrm{~mm}$ \\
\hline Outer diameter & $125 \mathrm{~mm}$ & Inner diameter & $30 \mathrm{~mm}$ \\
\hline Eigen-frequencies (numerical) & \multicolumn{3}{|c|}{$1195,1255,1361,1570,1665,1714,1756 \ldots \mathrm{Hz}$} \\
\hline $\begin{array}{c}\text { Acoustics eigen-frequencies considering ASI } \\
\text { (numerical) }\end{array}$ & \multicolumn{3}{|c|}{$1196,1255,1360,1570,1665,1714,1756 \ldots \mathrm{Hz}$} \\
\hline
\end{tabular}

Also, the prototype of the helicoidal resonator which is made by a 3D printer at the University of Twente is exposed in Fig. 5.2:
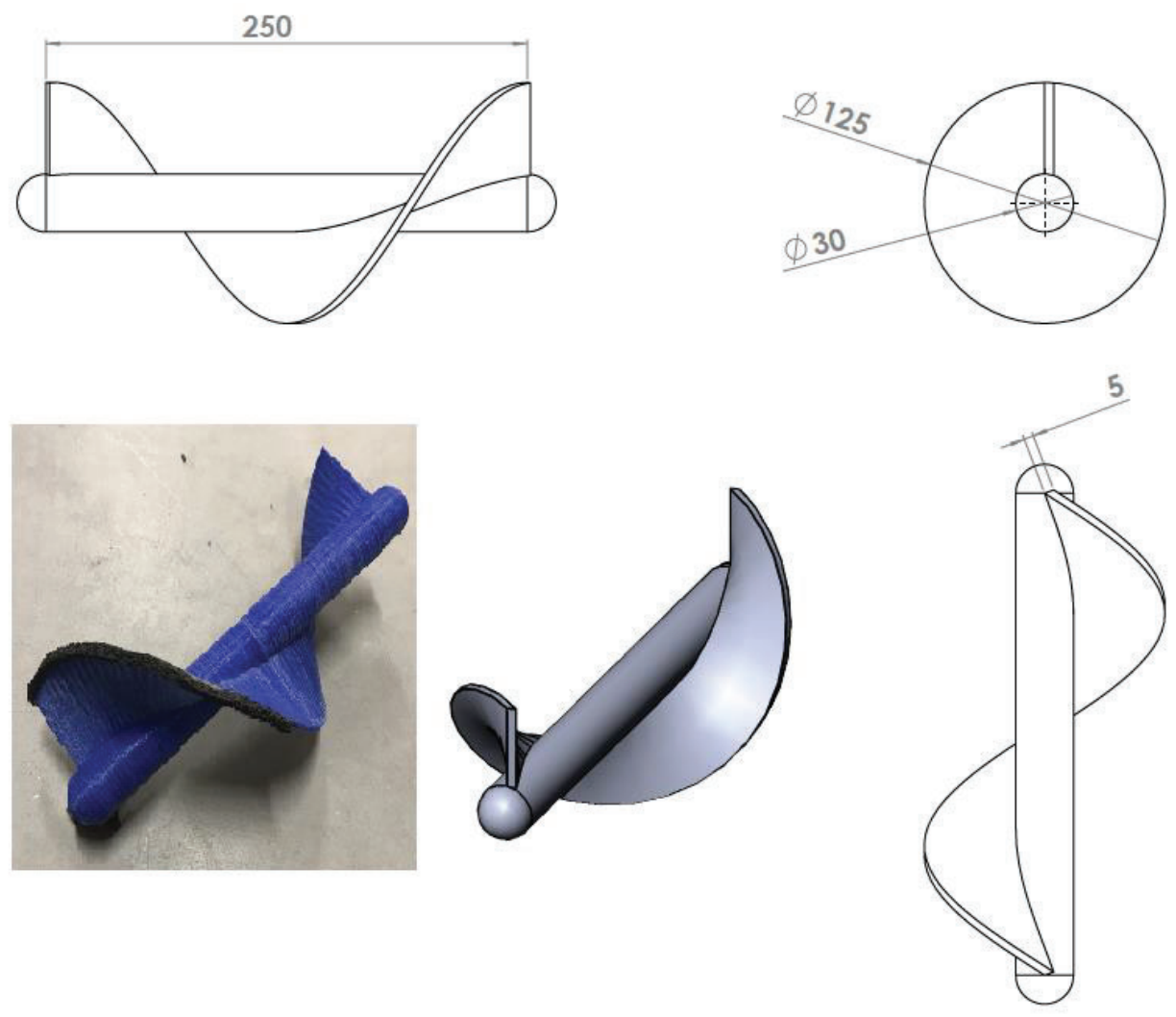

Figure 5.2: Detail design of the helicoidal resonator. 


\subsection{Spiral resonator}

The spiral resonator with three turns and $500 \mathrm{~mm}$ length is considered for the prototype. The specification of the prototype is written in Table 5.2.

Table 5.2: Specification of the spiral resonator.

\begin{tabular}{|c|c|c|c|}
\hline & \multicolumn{4}{|c|}{ Spiral resonator } & & \\
\hline Length & $500 \mathrm{~mm}$ & Blade thickness & $0.5 \mathrm{~mm}$ \\
\hline Outer diameter & $125 \mathrm{~mm}$ & No. of spiral turns & 3 \\
\hline Material & Stainless steel & Half-closed end \\
\hline Eigen-frequencies (numerical) & $430,629,700,826,850,978,1012,1028 \mathrm{~Hz}$ \\
\hline
\end{tabular}

The various views of the spiral resonator are plotted in Fig. 5.3.
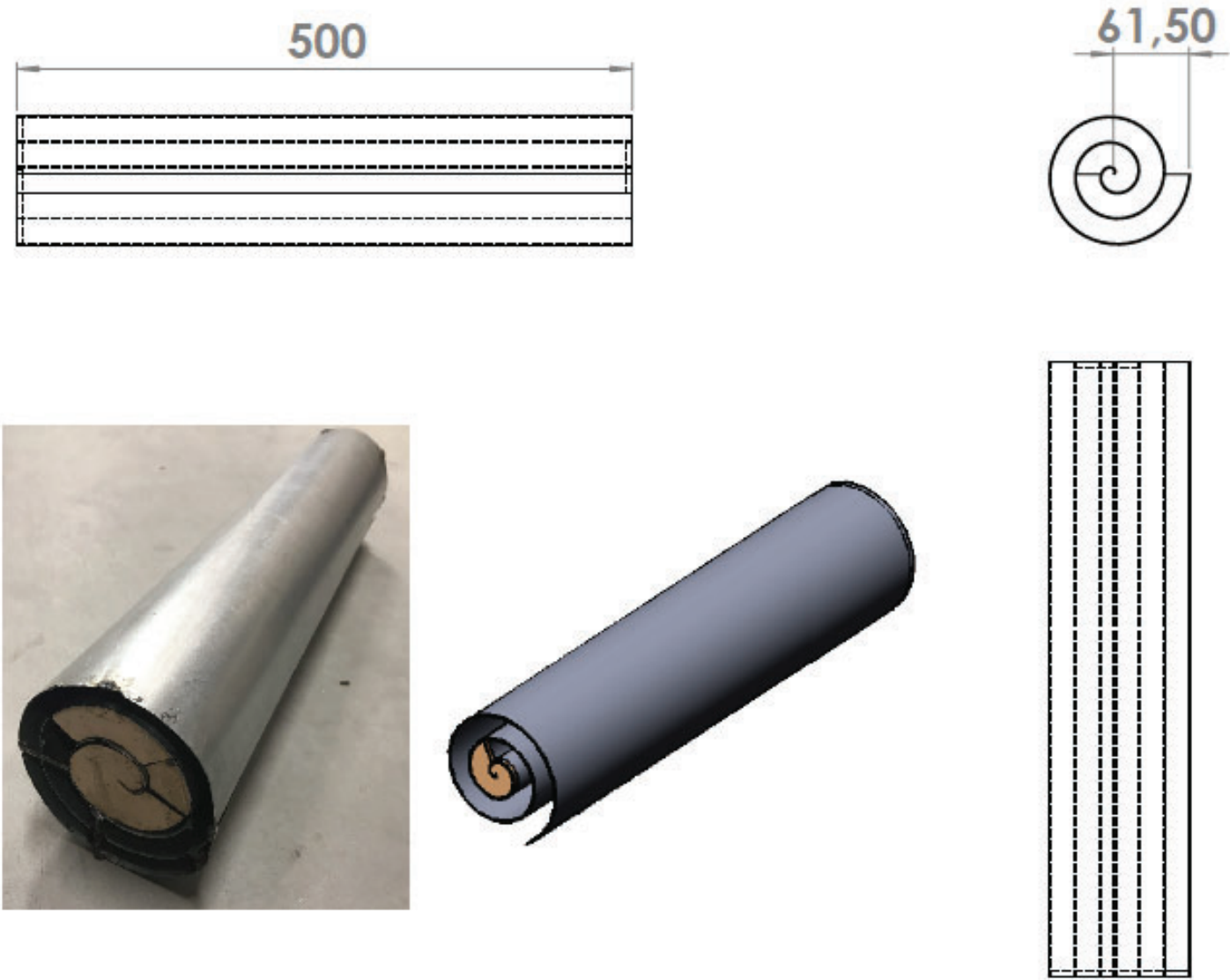

Figure 5.3: Detail design of the spiral resonator. 


\subsection{Funnel resonator}

The funnel resonator is useful for low-frequency noise reduction. The model with the written specification in Table 5.3, is made at Brink Climate Systems to evaluate the acoustic performance of the funnel resonator.

Table 5.3: Specification of the spiral resonator.

\begin{tabular}{|c|c|c|c|}
\hline \multicolumn{4}{|c|}{ Funnel resonator } \\
\hline Length & $600 \mathrm{~mm}$ & Blade thickness & $1 \mathrm{~mm}$ \\
\hline Inlet diameter & $60 \mathrm{~mm}$ & Outlet diameter & $109.67 \mathrm{~mm}$ \\
\hline Material & Stainless steel & Slope & $4.1 \%$ \\
\hline Eigen-frequencies (analytical) & \multicolumn{3}{|c|}{$287,578,858,1147 \ldots \mathrm{Hz}$} \\
\hline Eigen-frequencies (numerical) & \multicolumn{3}{|c|}{$273,553,831,1063,1109 \mathrm{~Hz}$} \\
\hline
\end{tabular}

The SolidWorks model and the prototype of the funnel resonator is shown in Fig. 5.4.
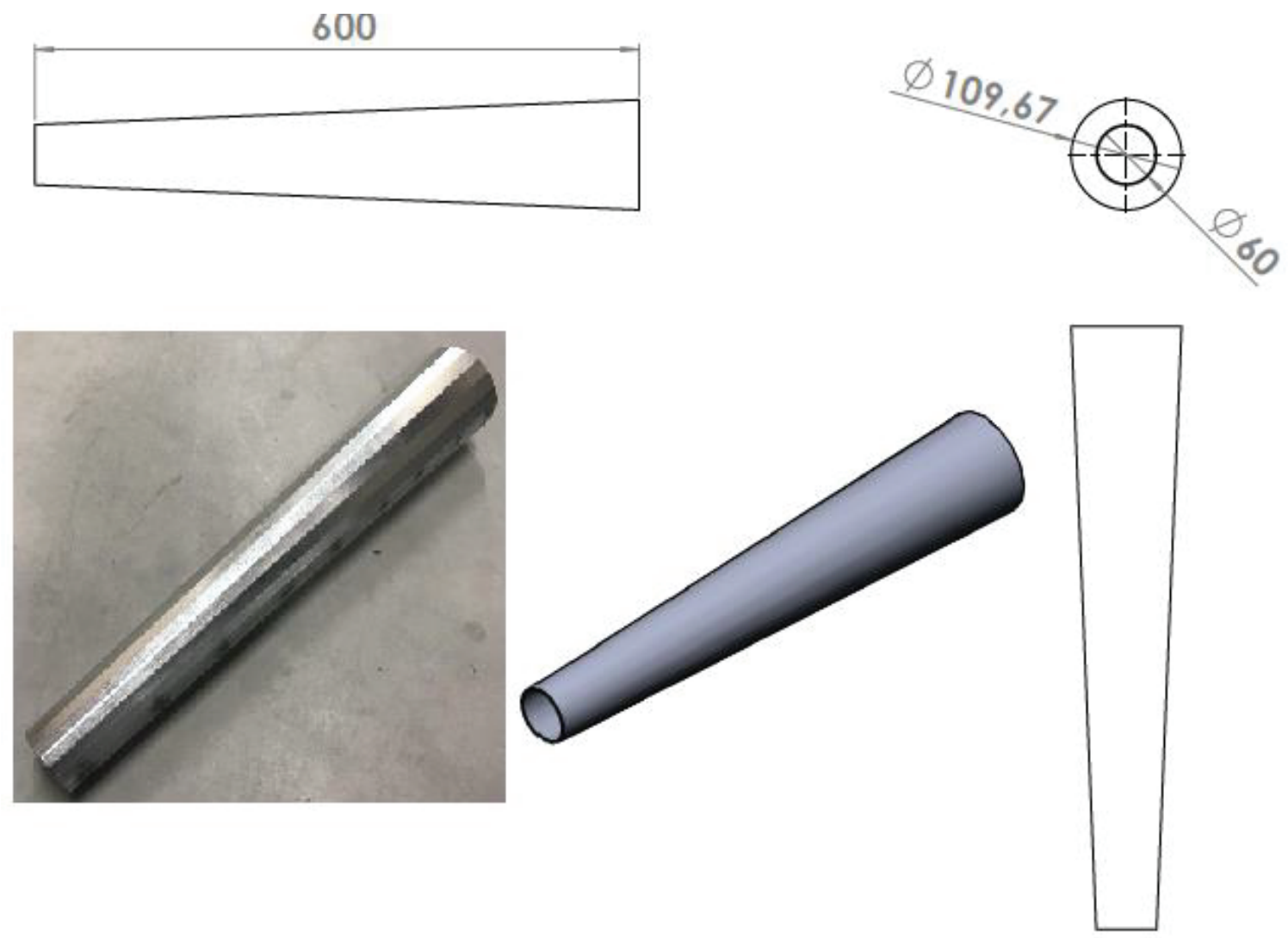

Figure 5.4: Detail design of the funnel resonator. 


\subsection{Acoustic Black Hole muffler}

Because of the Acoustic Black Hole muffler should be installed inside the T-joint, then the outer diameter size is $125 \mathrm{~mm}$. The model which is designed in this section has the written specifications mentioned in Table. 5.4.

Table 5.4: Specification of the Acoustic Black Hole muffler.

\begin{tabular}{|c|c|c|c|}
\hline \multicolumn{4}{|c|}{ Acoustic Black Hole muffler } \\
\hline Length & $135 \mathrm{~mm}$ & Blade thickness & $1 \mathrm{~mm}$ \\
\hline No. of blades & 18 & Outlet diameter & $125 \mathrm{~mm}$ \\
\hline Material & ABS & \\
\hline Eigen-frequencies (numerical) & $\begin{array}{c}\text { 285, } 433,609,731,834,941,1055,1178,1306,1452,1610 \\
1619,1620,1624,1631,1632 \ldots \mathrm{Hz}\end{array}$ \\
\hline
\end{tabular}

The standard view of the model and the produced prototype is plotted in Fig. 5.5:
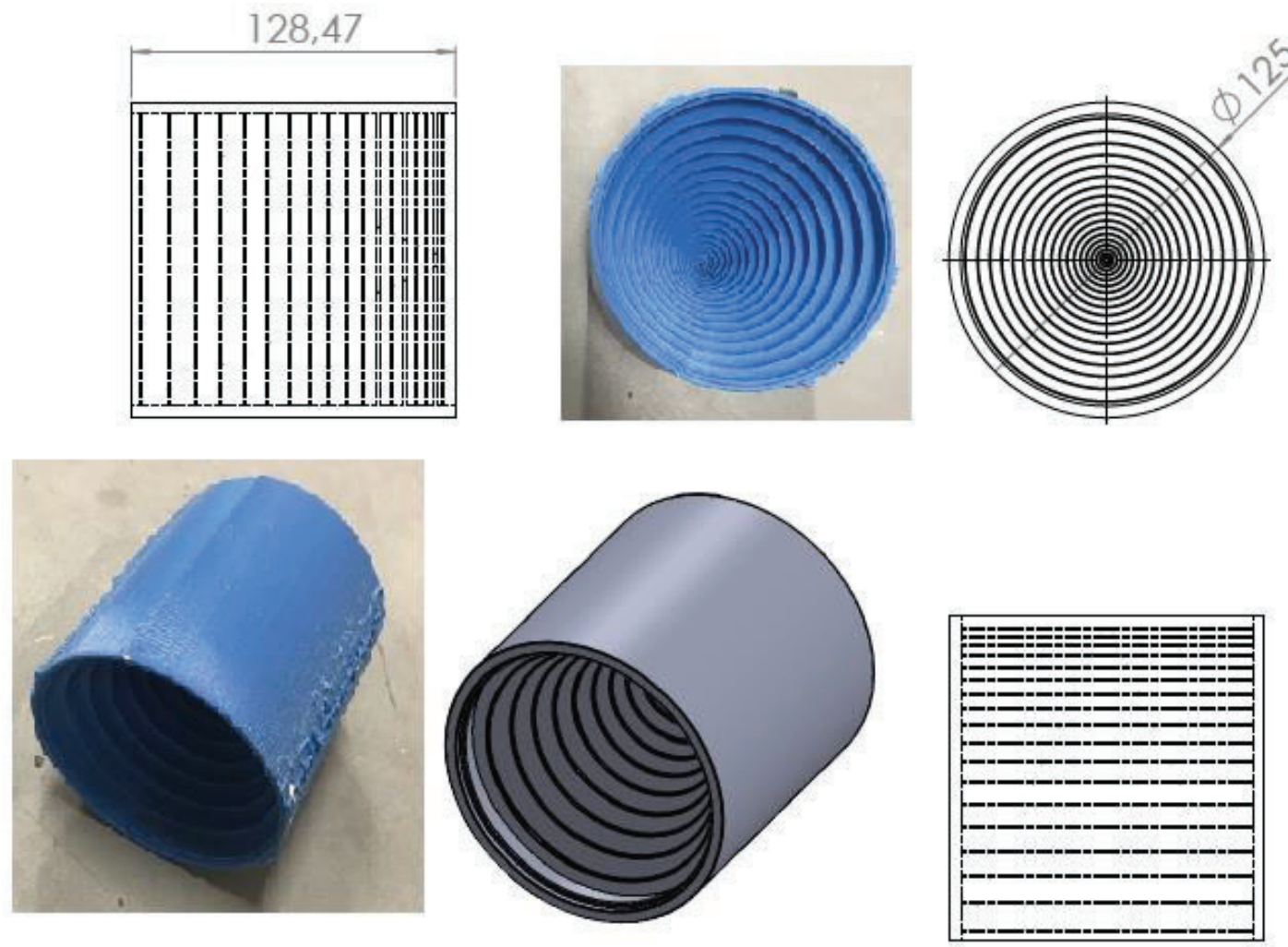

Figure 5.5: Detail design of the Acoustic Black Hole muffler. 


\subsection{External Acoustic Black Hole muffler}

Based on the requirement, the outer diameter of the muffler size is selected to be $254 \mathrm{~mm}$ and the inner one should be the same as duct size which is $125 \mathrm{~mm}$. By adding more channels inside the muffler, the transmission loss function will be smoother and higher. However, the smallest possible thickness to make is $0.4 \mathrm{~mm}$ and the channel width should be more than 1 $\mathrm{mm}$ to be possible to make it with 3D printer at University of Twente (model 'FORMIGA P101'). The specification of the EABH muffler outside the ventilation unit is written in Table 5.5:

Table 5.5: Specification of the external Acoustic Black Hole muffler.

\begin{tabular}{|c|c|c|c|}
\hline & External Acoustic Black Hole muffler \\
\hline Length & $182 \mathrm{~mm}$ & Wall thickness & $1 \mathrm{~mm}$ \\
\hline No. of channels & 24 & Outer diameter & $254 \mathrm{~mm}$ \\
\hline Material & Polyamide 2200 & Inner diameter & $125 \mathrm{~mm}$ \\
\hline Eigen-frequencies & $232,242,253,264,278,294,311,327,334,361,392,432,550,607,644$, \\
\hline (numerical) & $681,718,723,767,786,819,866,871,941,971,978,1015 \ldots . \mathrm{Hz}$ \\
\hline
\end{tabular}

One of the best possible model as the external Acoustic Black Hole muffler is shown in Fig.

5.6 .

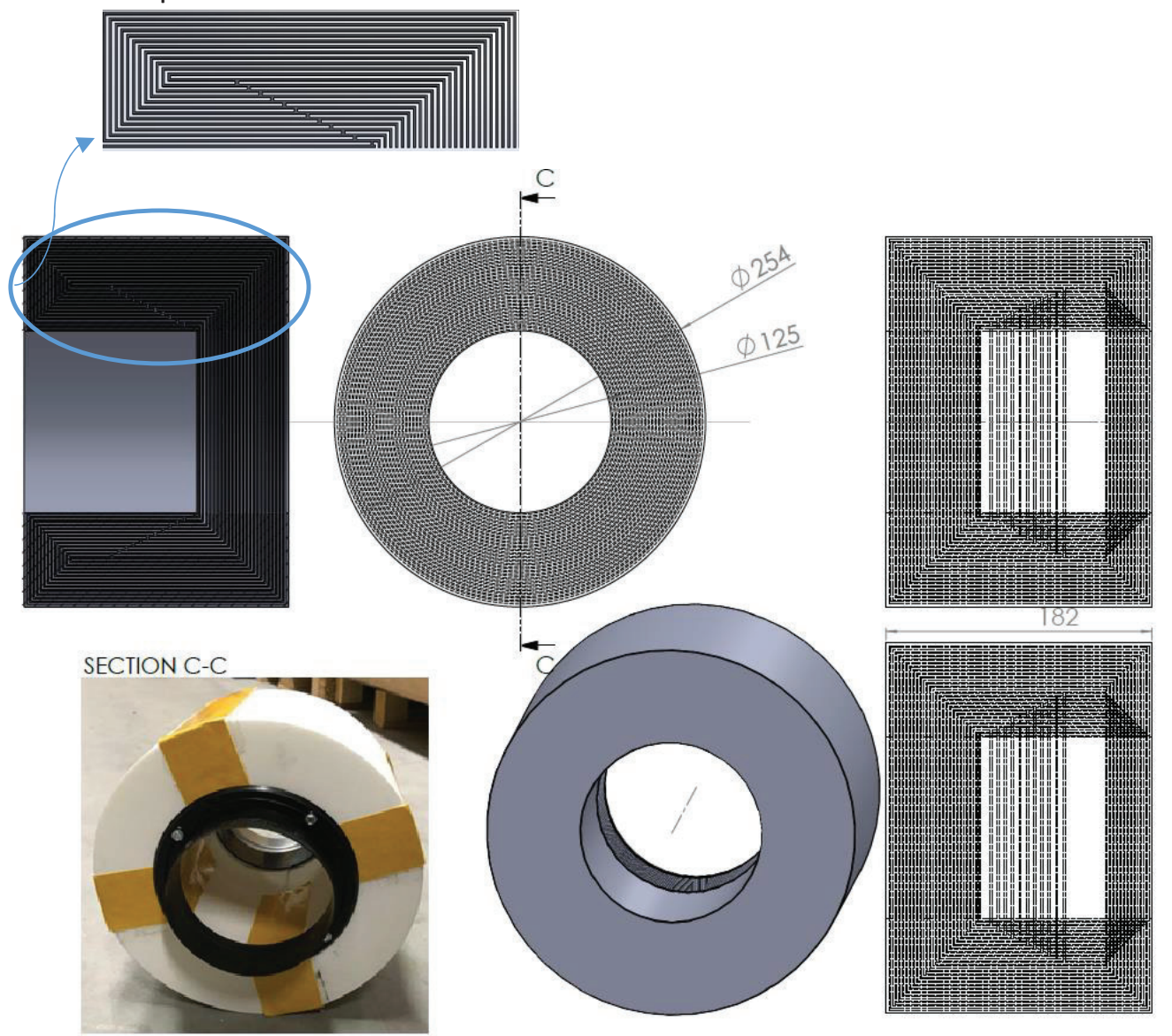

Figure 5.6: A detail design of the external Acoustic Black Hole outside the ventilation unit. 
The muffler is divided into four segments, shown in Fig 5.7, which could be possible to make them with the mentioned 3D printer.

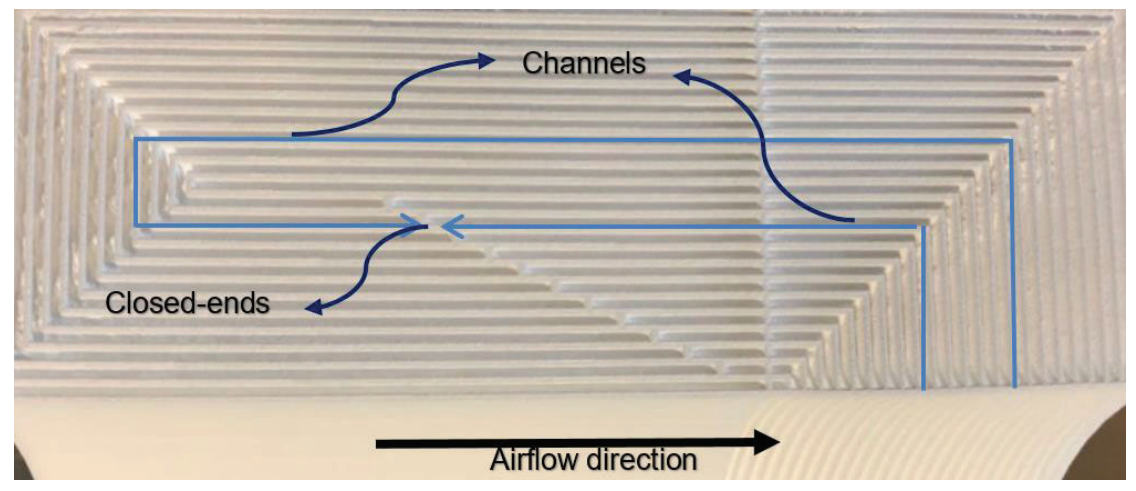

Figure 5.7: A segment of the external Acoustic Black Hole muffler outside the ventilation unit.

\subsection{Internal Acoustic Black Hole muffler}

As discussed before, to put the silencer inside the diffuser of the ventilation unit, it is needed to make some segments. These segments should have enough rigidity and should be possible to make by injection molding technique. Hence, a wall is inserted in the middle and the blade should be extruded with at least $1^{\circ} \mathrm{draft}$. Also, it needs some pins which connect them together. Based on these characteristics, the designed segment is presented in Fig. 5.8:

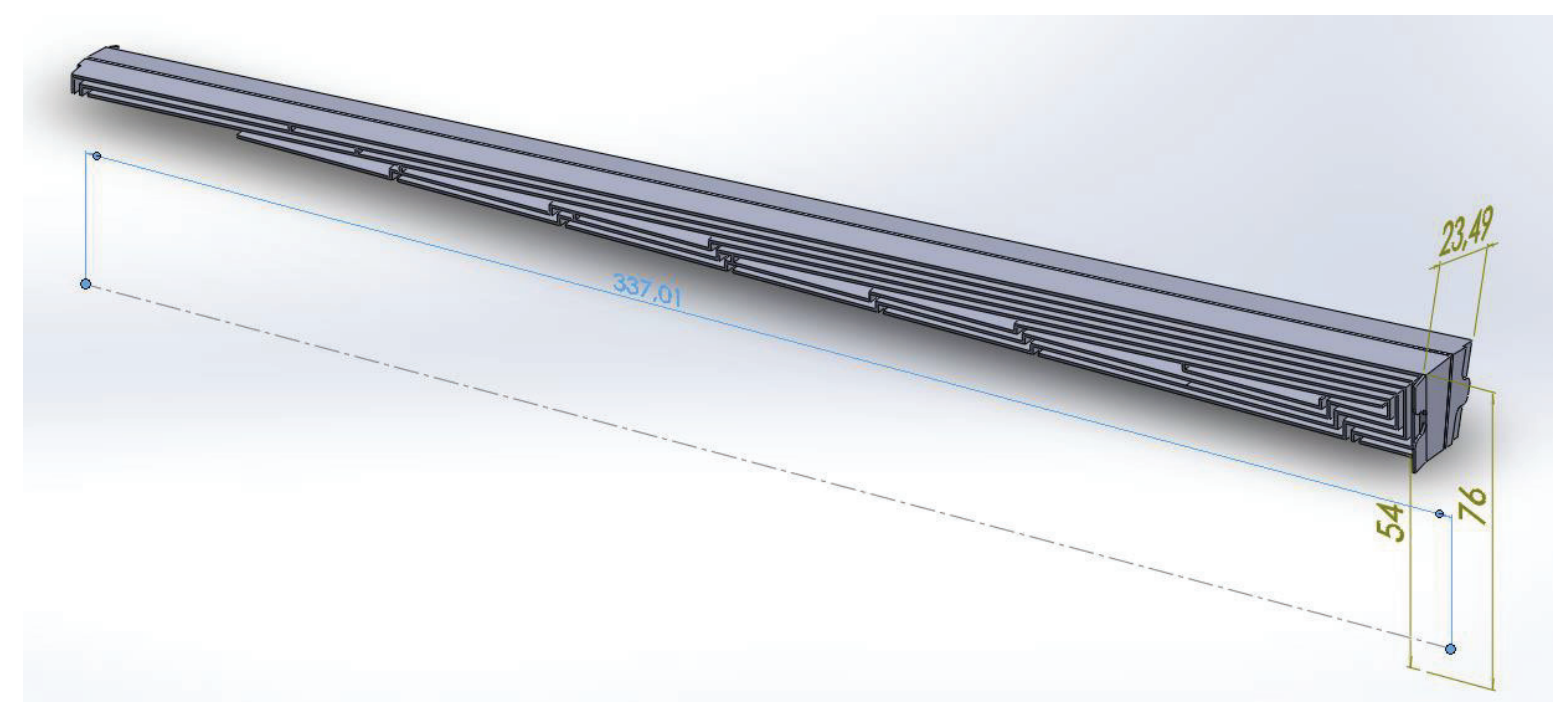

Figure 5.8: A segment of the internal Acoustic Black Hole inside the ventilation unit. 
The specification of this design is depicted in Table 5.6:

Table 5.6: Specification of the internal Acoustic Black Hole muffler inside the unit.

\begin{tabular}{|c|c|c|c|}
\hline \multicolumn{4}{|c|}{ Internal Acoustic Black Hole muffler } \\
\hline Length & $337 \mathrm{~mm}$ & Wall thickness & $0.6-1 \mathrm{~mm}$ \\
\hline No. of channels & 10 & Outer diameter & $118-152 \mathrm{~mm}$ \\
\hline Material & Polyamide 2200 & Inner diameter & $108 \mathrm{~mm}$ \\
\hline Volume & $41 \mathrm{dm}^{3}$ & General draft & 1 degree \\
\hline $\begin{array}{l}\text { Eigen-frequencies } \\
\text { (numerical) }\end{array}$ & \multicolumn{3}{|c|}{$111,139,179,203,207,238,271,289,295,399,498,528 \mathrm{~Hz}$} \\
\hline $\begin{array}{l}\text { Acoustics Eigen-frequencies } \\
\text { considering ASI (numerical) }\end{array}$ & \multicolumn{3}{|c|}{$\begin{array}{c}180,181,187,190,210,211,232,235,245,247,374,375 \\
381,382,394,395,397,399,403 \mathrm{~Hz}\end{array}$} \\
\hline
\end{tabular}

The Cad-drawing of the segment to get an offer from the suppliers is shown in Fig. 5.9:

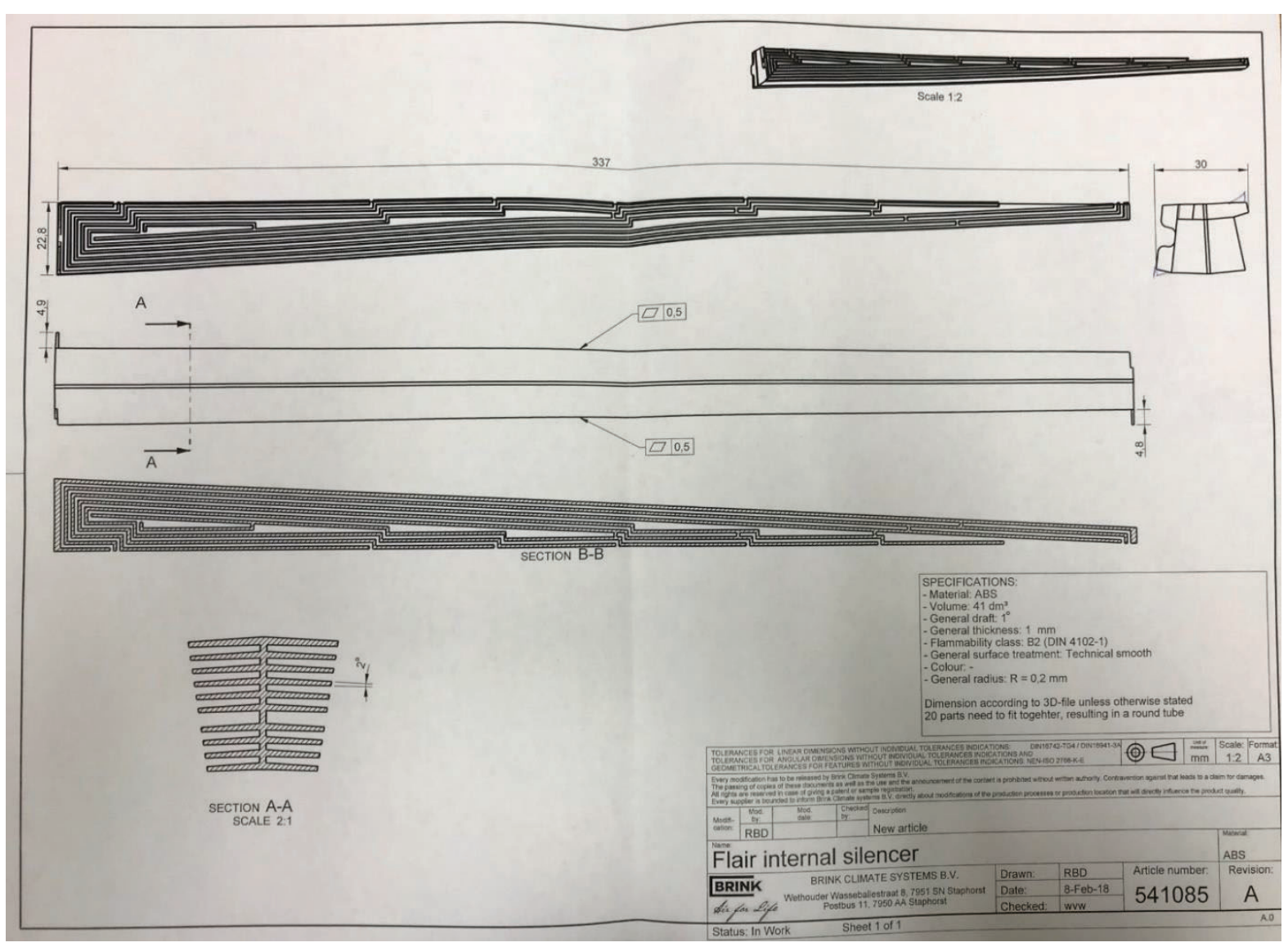

Figure 5.9: The Cad-drawing of a segment of the internal Acoustic Black Hole muffler.

The inserted pins help to assemble the segments like in Fig. 5.10: 


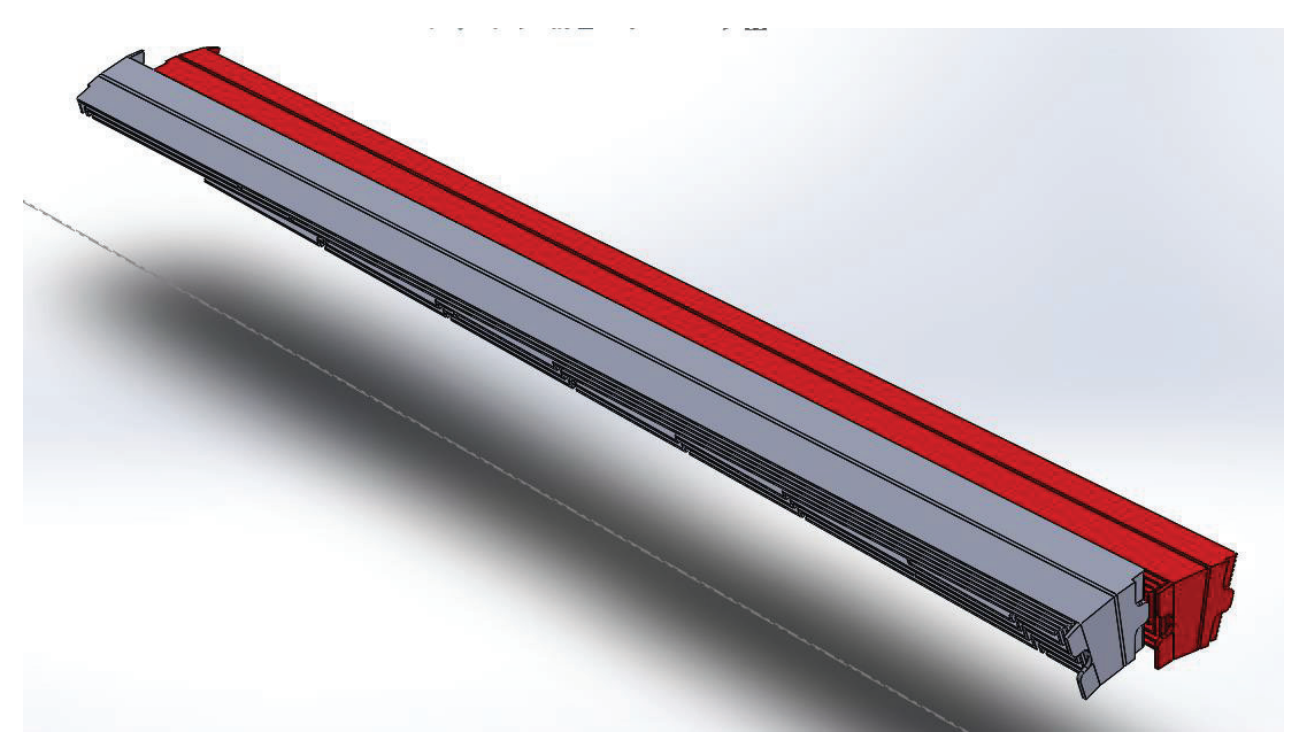

Figure 5.10: Assembly of segments of the internal Acoustic Black Hole muffler.

The final model of the muffler will become like in Fig. 5.11:

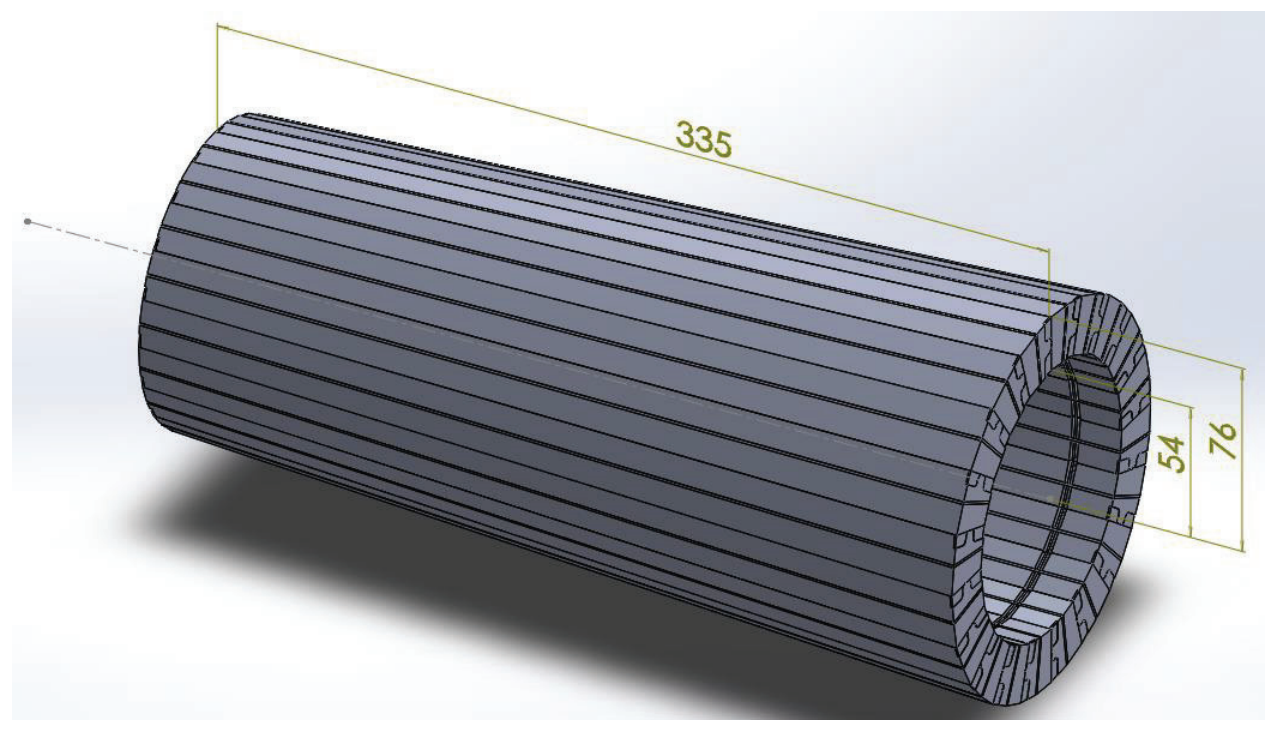

Figure 5.11: The internal Acoustic Black Hole muffler inside the ventilation unit.

Due to the maximum possible length of parts that 3D printer (model: FORMIGA P101) at the university could print, each segment is cut into two parts, then these parts are glued to become a segment like in Fig. 5.12: 

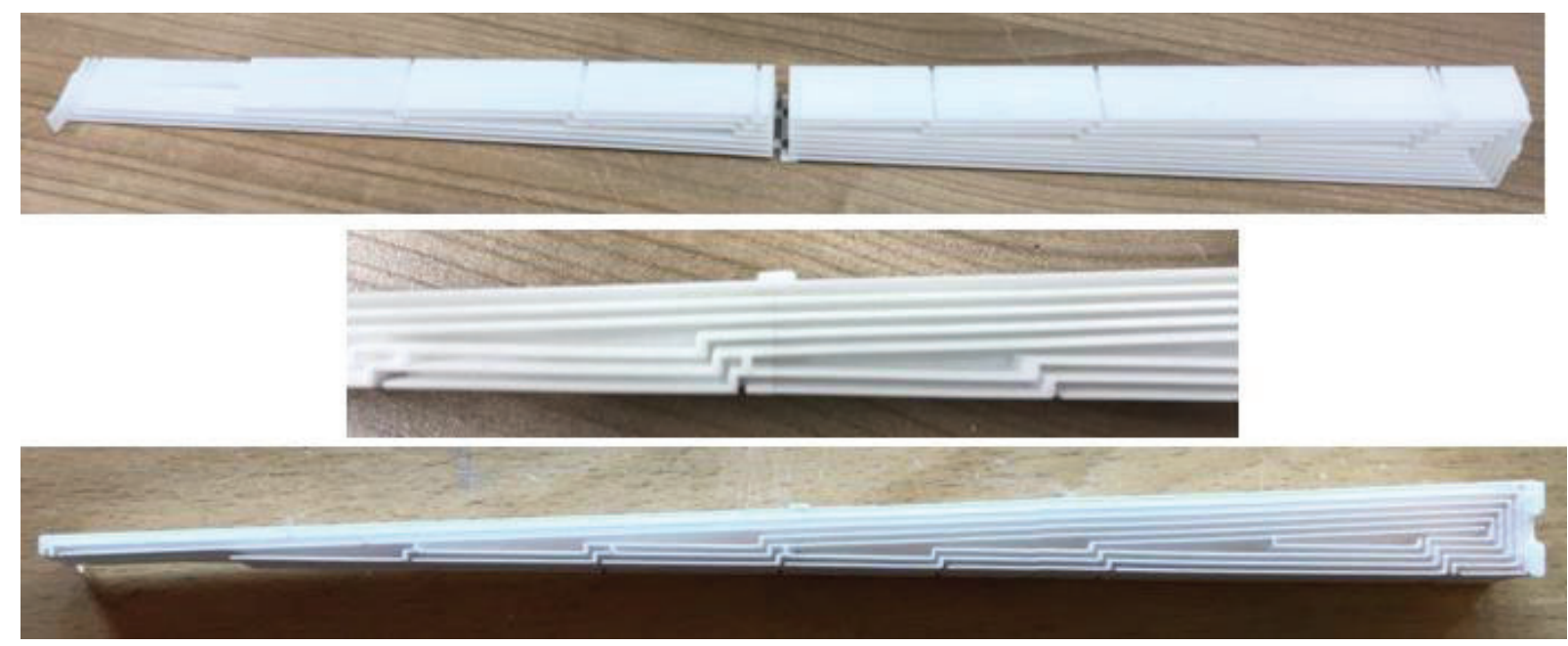

Figure 5.12: Making of each segments.

To assemble the muffler easily, two connectors are used like in Fig. 5.13 which gather the segments somehow they can be taped.
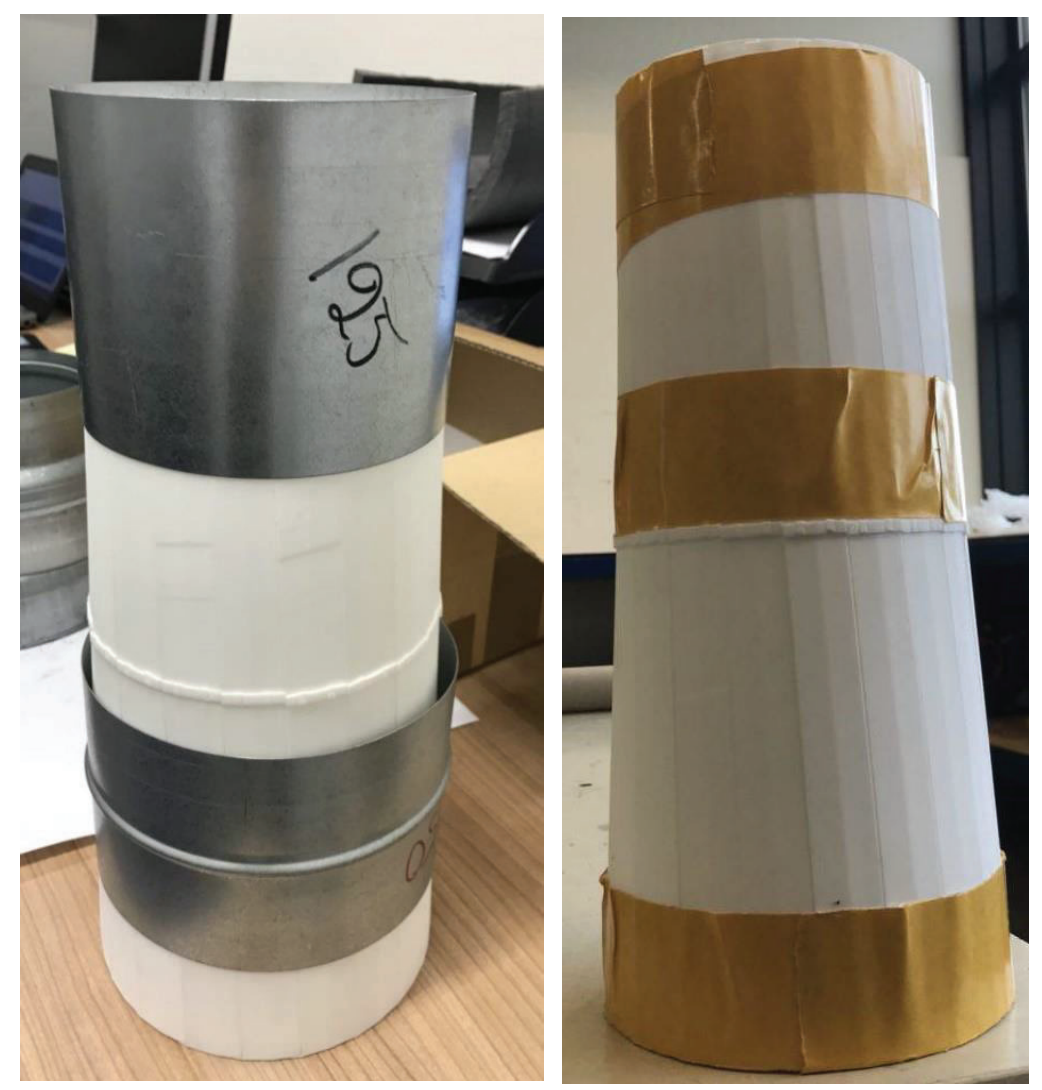

Figure 5.13: The prototype of the internal Acoustic Black Hole muffler.

In short, the detail of each treatments are presented in this chapter and prototypes are prepared to test them which is explained in the next chapter. 


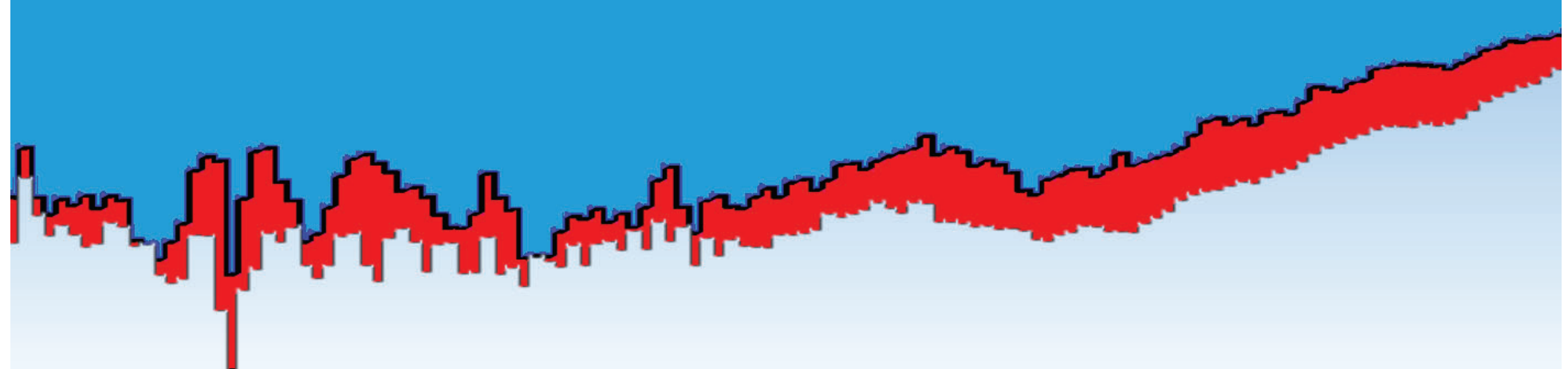

\section{Chapter 6}

\section{Treatment Implementation}

\subsection{Test setup}

The test setups are made at the Brink Climate Systems. The company has a reverberation chamber to measure sound Power Level of the ventilation units. In this chamber, six microphones, model: Bruel \& Kjaer 2671, are located to measure the acoustical pressure in 3 sets of 10 seconds based on ISO 3741 standard. In addition, it's equipped with a data acquisition system, model: Bruel \& Kjaer 3050-A-06, to transfer the acoustic pressure data to the computer. Two test setups are prepared to evaluate the resonators and mufflers in a controlled environment and a real environment whether the requirement of the stakeholders are met.

The first test setup shown in Fig. 6.1, it involves the ventilation unit's fan as a noise source, model: ebmpapst G3G190, a flow meter to adjust the flowrate, model: Flakt 125, pitot tubes to measure the pressure drop, a micro-manometer, model: RS232, 6 microphones (5 located in the reverberation chamber and another one is placed before the resonator inside the tube) to measure the acoustic pressures.
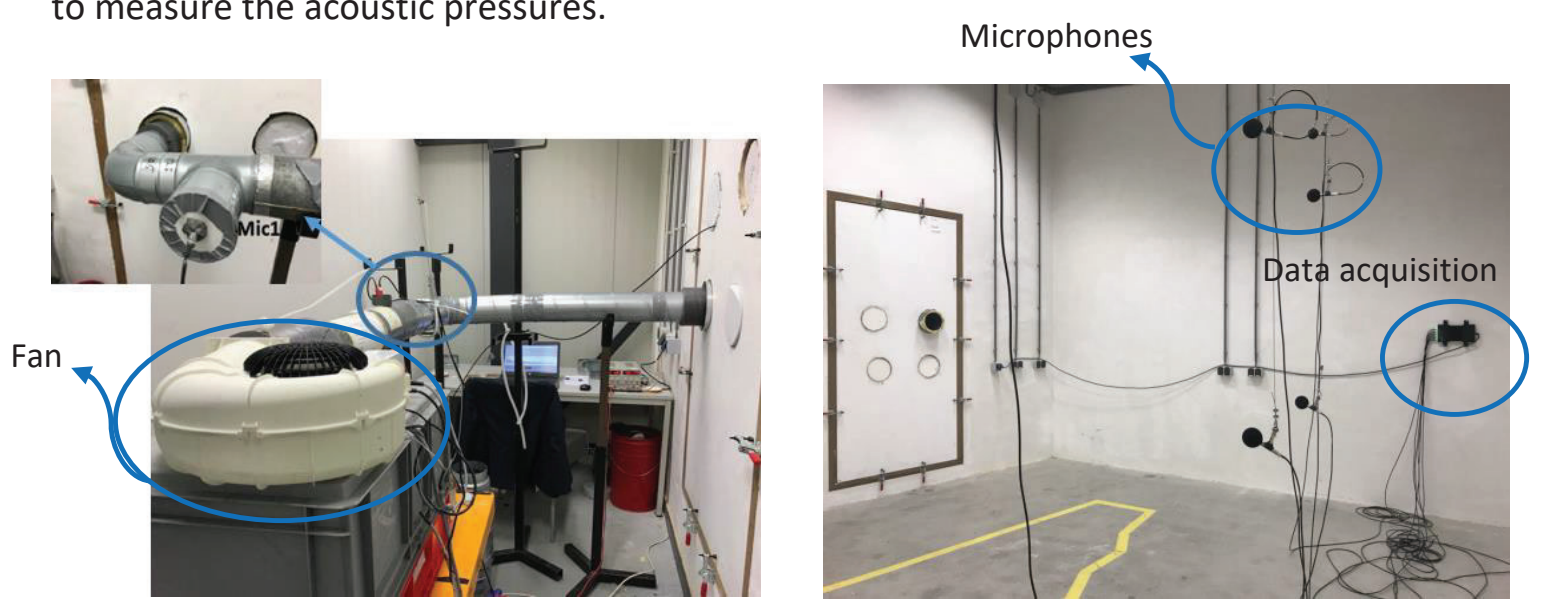

Figure 6.1: First test setup for testing in the controlled environment. 
The second setup configuration is used for testing the resonators and mufflers inside and outside the ventilation unit in the real environment which is presented in Fig. 6.2 and sound power level is measured by six microphones inside the reverberation chamber.
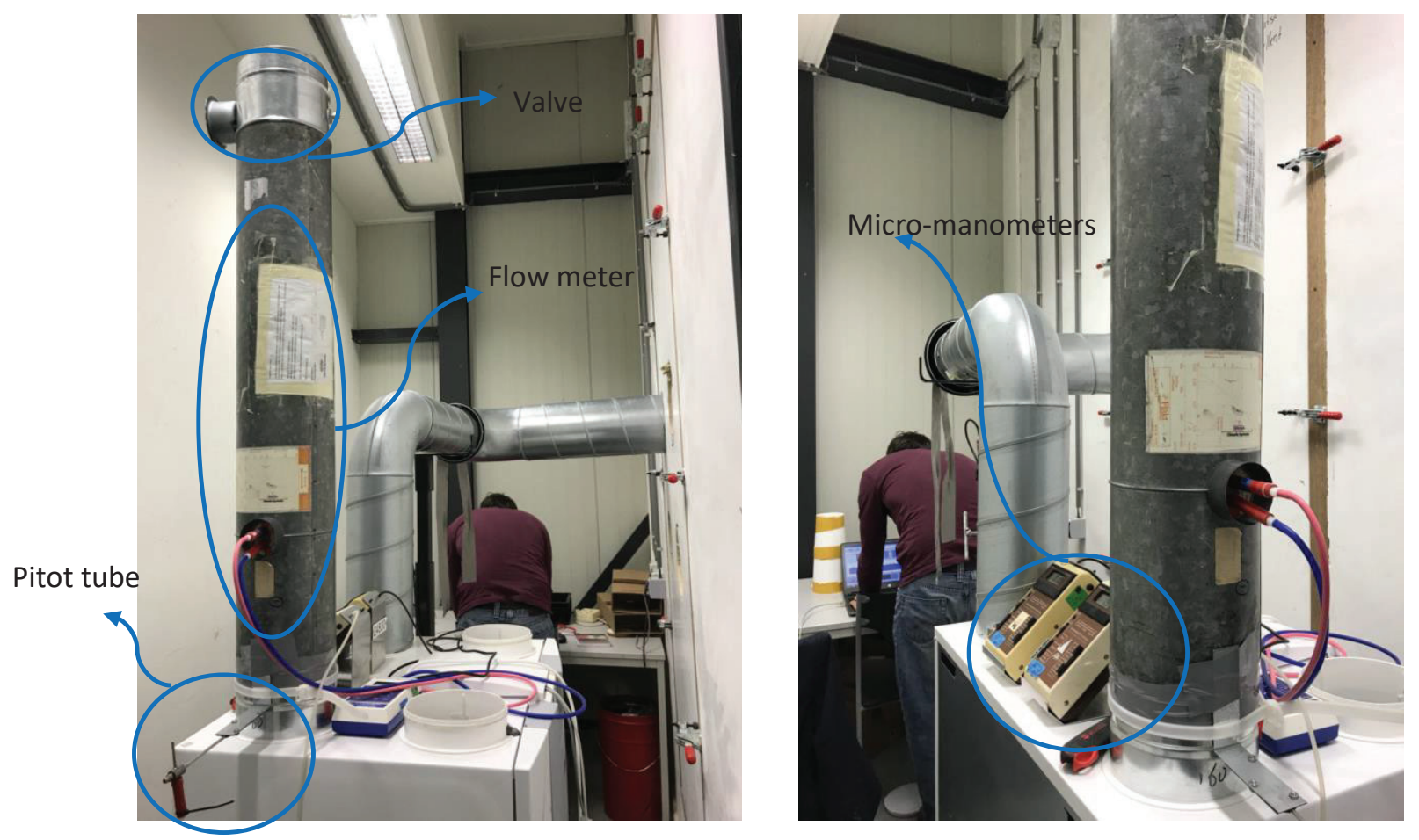

Figure 6.2: Second test setup for testing in the real environment.

The second test setup also involves the flow meter, three Pitot tubes to measure the pressure drop between the inlet and outlet ducts and pressure drop of the resonators, a power-meter, model: Christ CLM1000, a valve to adjust the pressure at the inlet duct, and the ventilation unit of Brink Climate Systems, model: Flair 325, shown in Fig. 6.3:

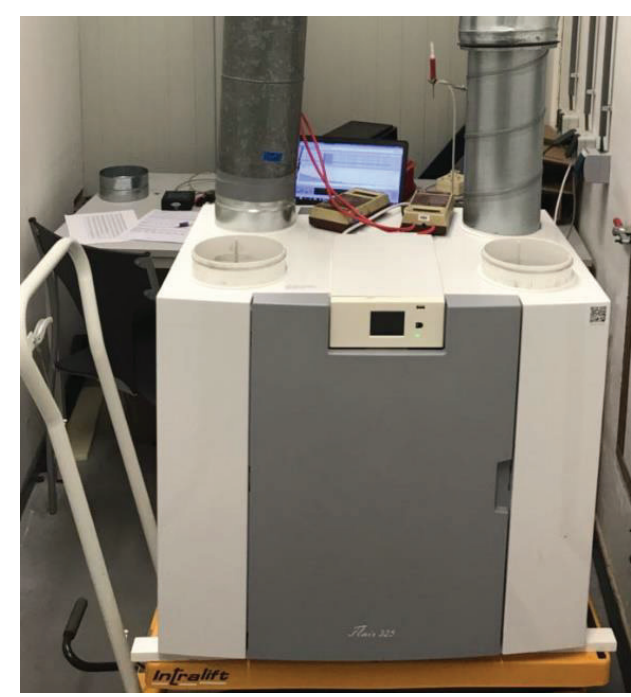

Figure 6.3: A ventilation unit of Brink Climate Systems model Flair 325. 
Moreover, the internal resonators like the helicoidal resonator, decrease the cross section of the tube which is causing more pressure drop, then the fan needs to work harder and it makes more noise. In this case, the performance of the resonator cannot be evaluated well due to different noise sources. Hence, the co-axial duct, shown in Fig. 6.4, is used and the insertion loss is calculated between the situation that the resonator has been installed inside the coaxial duct and the situation without the resonator.

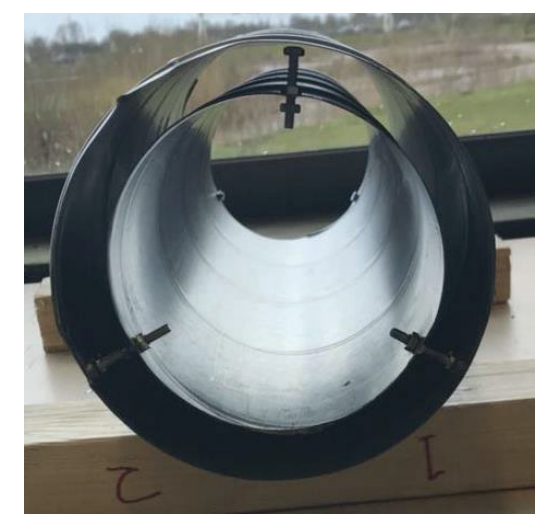

Figure 6.4: The co-axial duct (outer diameter: $160 \mathrm{~mm}$, inner diameter: $125 \mathrm{~mm}$ ).

For instance, the $1 / 1$ and $1 / 3$ octave band sound power level spectrum of the Flair 325 without any resonator at $325 \mathrm{~m}^{3} / \mathrm{h}$ flowrate and $150 \mathrm{~Pa}$ pressure differences between the inlet and outlet ducts is plotted in Fig. 6.5. The weather conditions during the test were: temperature $19.9^{\circ} \mathrm{C}$, atmosphere pressure $1013.4 \mathrm{~Pa}$, and relative humidity $39 \%$.

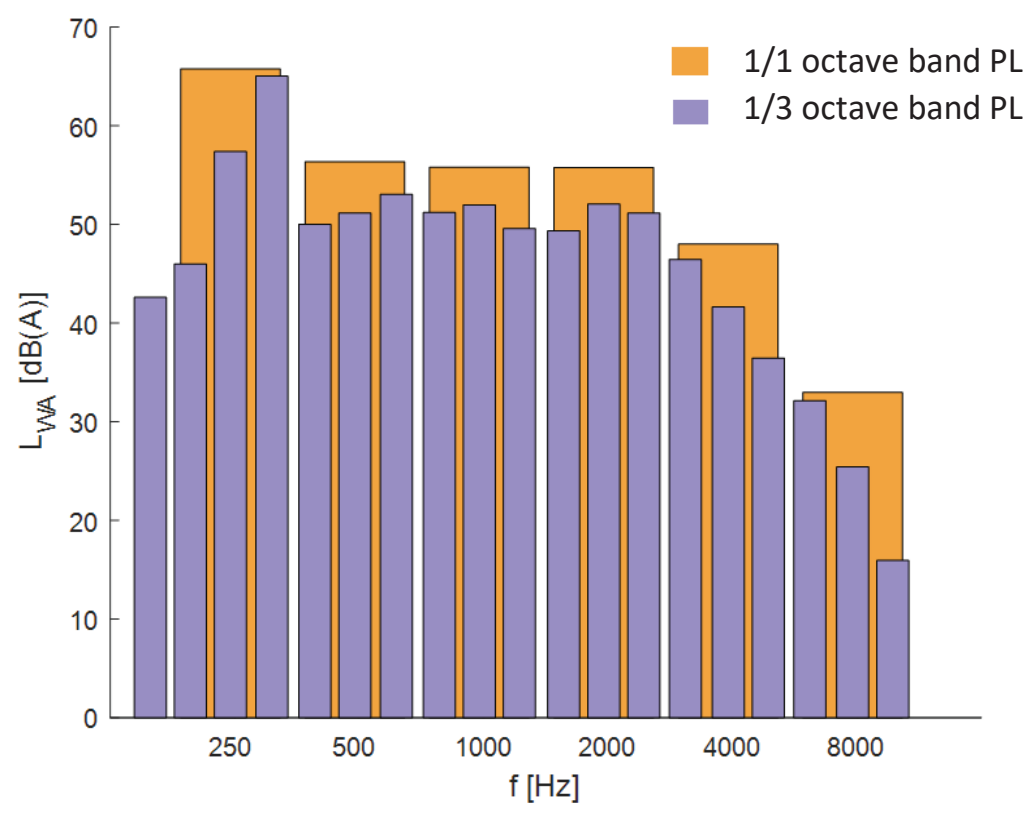

Figure 6.5: $1 / 1$ and 1/3 octave band sound power level spectrum of the unit (model: Flair 325). 
To have better analysis the result, the $1 / 24$ octave band PL spectrum is also considered. Fig. 6.6 shows the $1 / 24$ octave band sound power level spectrum of the Flair 325 without any resonator at $325 \mathrm{~m}^{3} / \mathrm{h}$ flowrate and 150 Pa pressure differences between the inlet and outlet ducts. Also, the frequency range is considered from $145 \mathrm{~Hz}$ to $12000 \mathrm{~Hz}$.

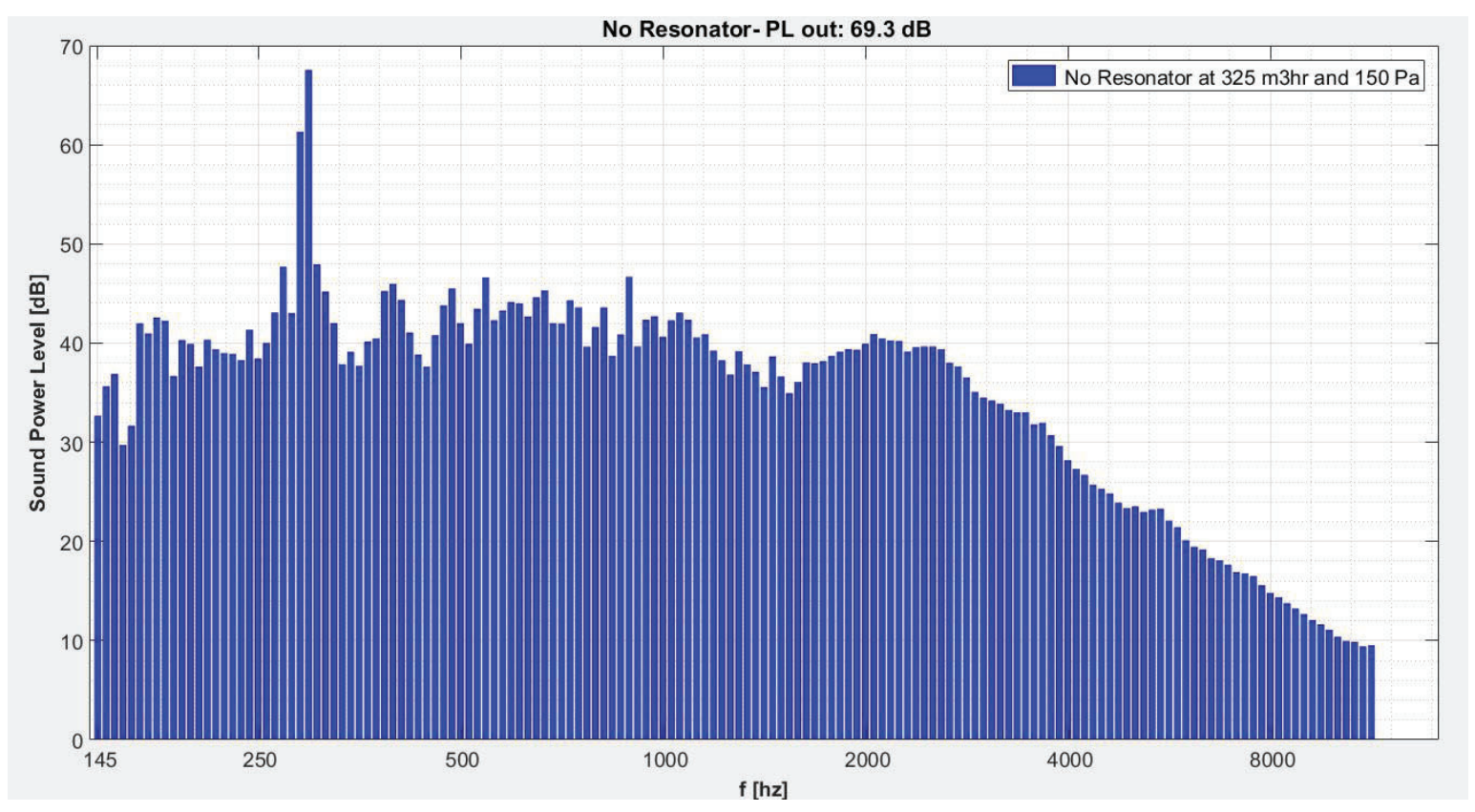

Figure 6.6: 1/24 octave band sound power level spectrum of the ventilation unit (model: Flair 325).

\subsection{Helicoidal resonator}

Fig. 6.7 presents the helicoidal resonator inside the co-axial ducts to install among the ventilation duct size $160 \mathrm{~mm}$.

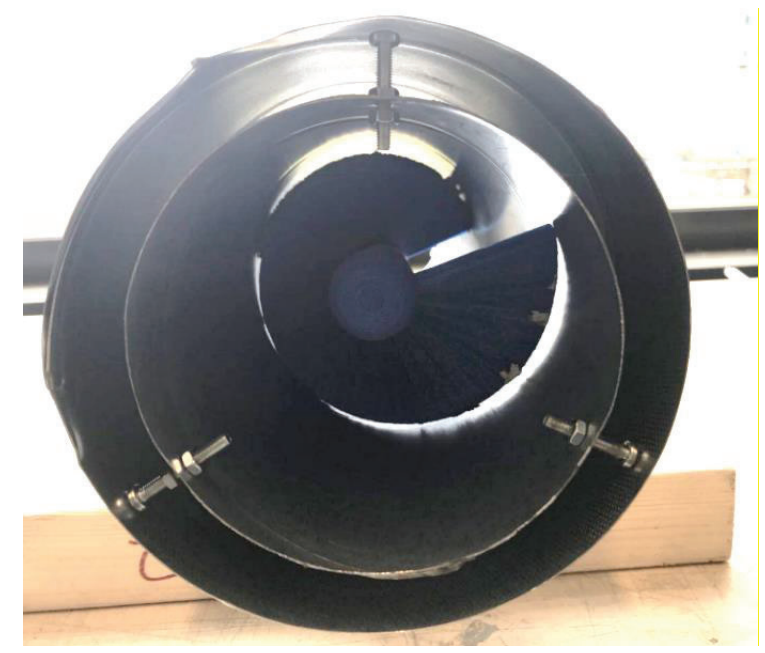

Figure 6.7. The helicoidal resonator inside the co-axial duct. 
The sound power level of the system by applying the helicoidal resonator inside the co-axial duct in the second test setup at $325 \mathrm{~m}^{3} / \mathrm{h}$ flowrate and $150 \mathrm{~Pa}$ pressure differences between the inlet and outlet ducts is shown in Fig. 6.8:

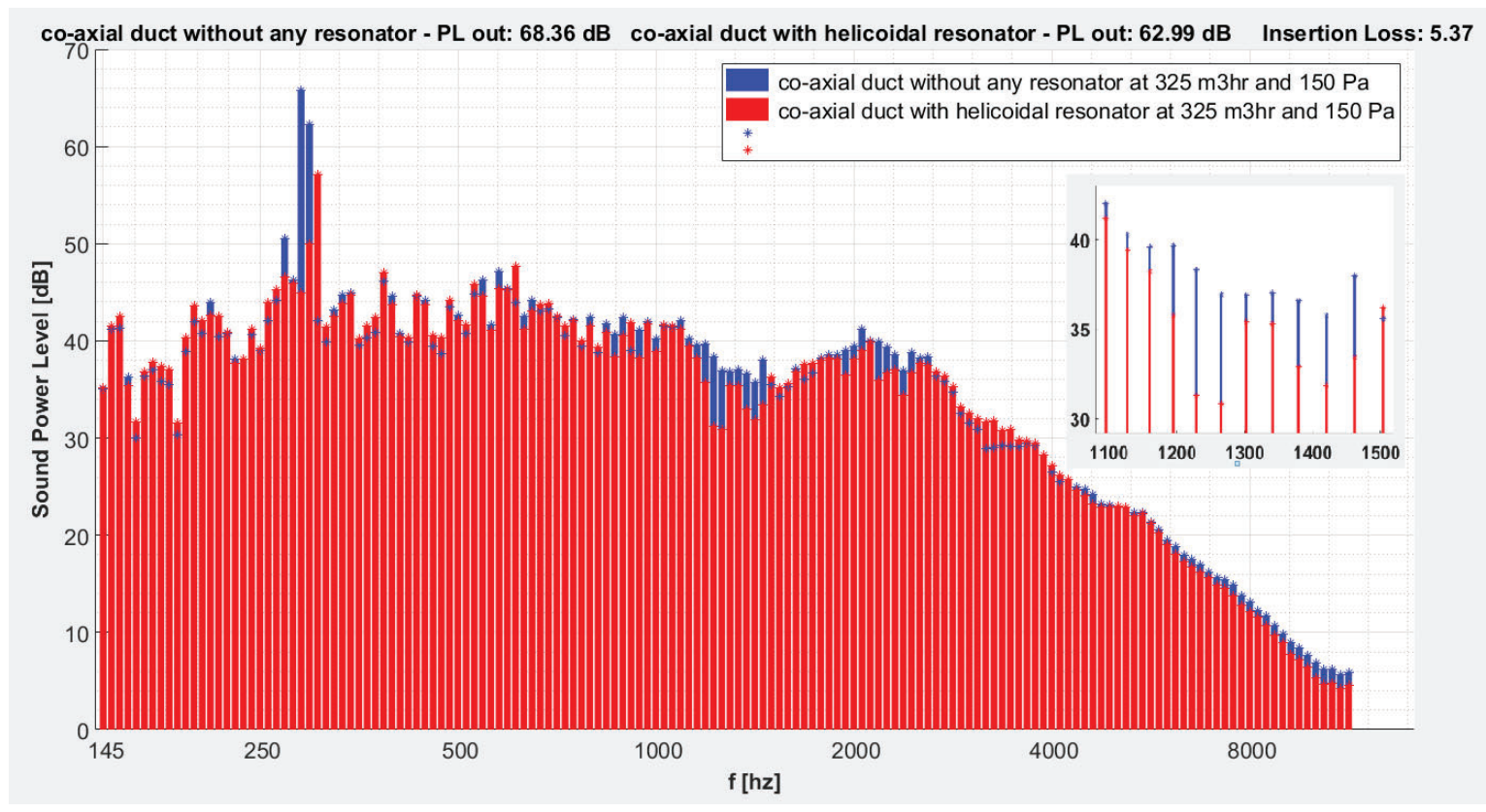

Figure 6.8: Sound power level of applying the helicoidal resonator at $325 \mathrm{~m}^{3} / \mathrm{h}$ and $150 \mathrm{~Pa}$.

As can be seen in Fig. 6.8, the noise reduction at the desired eigen-frequencies $(1180 \mathrm{~Hz}, 1300$ $\mathrm{Hz}, 1615 \mathrm{~Hz}, 1709 \mathrm{~Hz}$, etc.) are achieved. In addition, another analysis has been done at Brink to determine the sound power level of the unit at various frequencies and the result is written in Table. 6.1:

Table 6.1: measured Sound power level of the unit Flair 325.

\begin{tabular}{|c|c|c|c|c|c|c|c|}
\hline & Lw dB & Lw dB & Lw dB & Lw dB & Lw dB & Lw dB & Lwa $d B(A)$ \\
\hline & $250 \mathrm{~Hz}$ & $500 \mathrm{~Hz}$ & $1000 \mathrm{~Hz}$ & $2000 \mathrm{~Hz}$ & $4000 \mathrm{~Hz}$ & $8000 \mathrm{~Hz}$ & overall \\
\hline $\begin{array}{l}\text { co-axial } 160 \mathrm{~mm} \text { no silencer } \\
325 \mathrm{~m}^{3} / \mathrm{h}, 150 \mathrm{~Pa}\end{array}$ & 72.1 & 59.6 & 55.9 & 53.7 & 44.7 & 33.1 & 66.2 \\
\hline $\begin{array}{l}\text { co-axial } 160 \mathrm{~mm} \text { helicoidal } \\
\text { resonator } 325 \mathrm{~m}^{3} / \mathrm{h}, 150 \mathrm{~Pa}\end{array}$ & 66.2 & 59.7 & 55.2 & 52.7 & 45.5 & 32.5 & 62.6 \\
\hline Insertion Loss & 5.9 & -0.1 & 0.7 & 1 & -0.8 & -1.4 & 3.6 \\
\hline
\end{tabular}




\subsection{Spiral resonator}

The test setup configuration for the spiral resonator is the same as for the helicoidal resonator, the spiral resonator inside the co-axial duct is shown in Fig. 6.9:

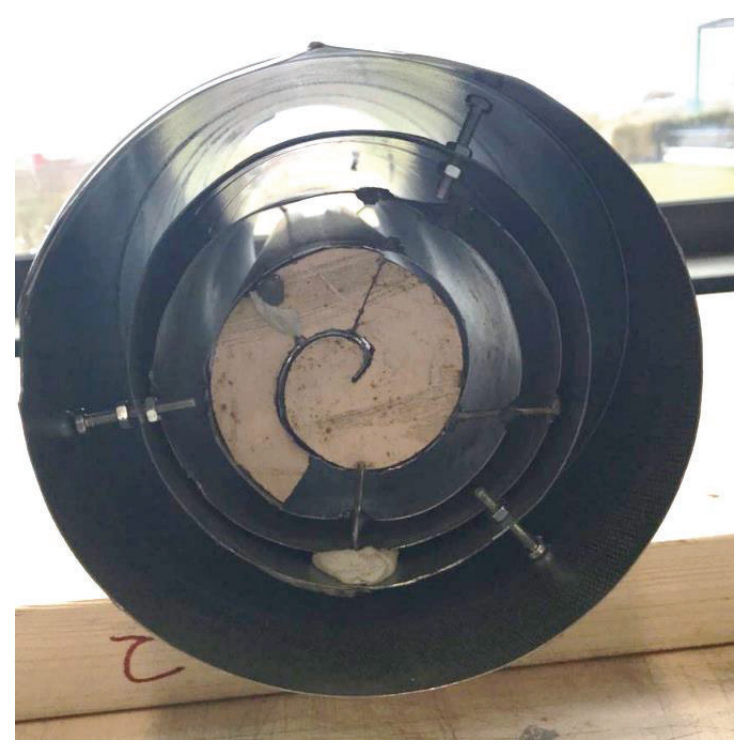

Figure 6.9. The spiral resonator inside the co-axial duct.

The sound power level of the spiral resonator at $325 \mathrm{~m}^{3} / \mathrm{h}$ flowrate and 150 Pa pressure over the unit is plotted in Fig. 6.10:

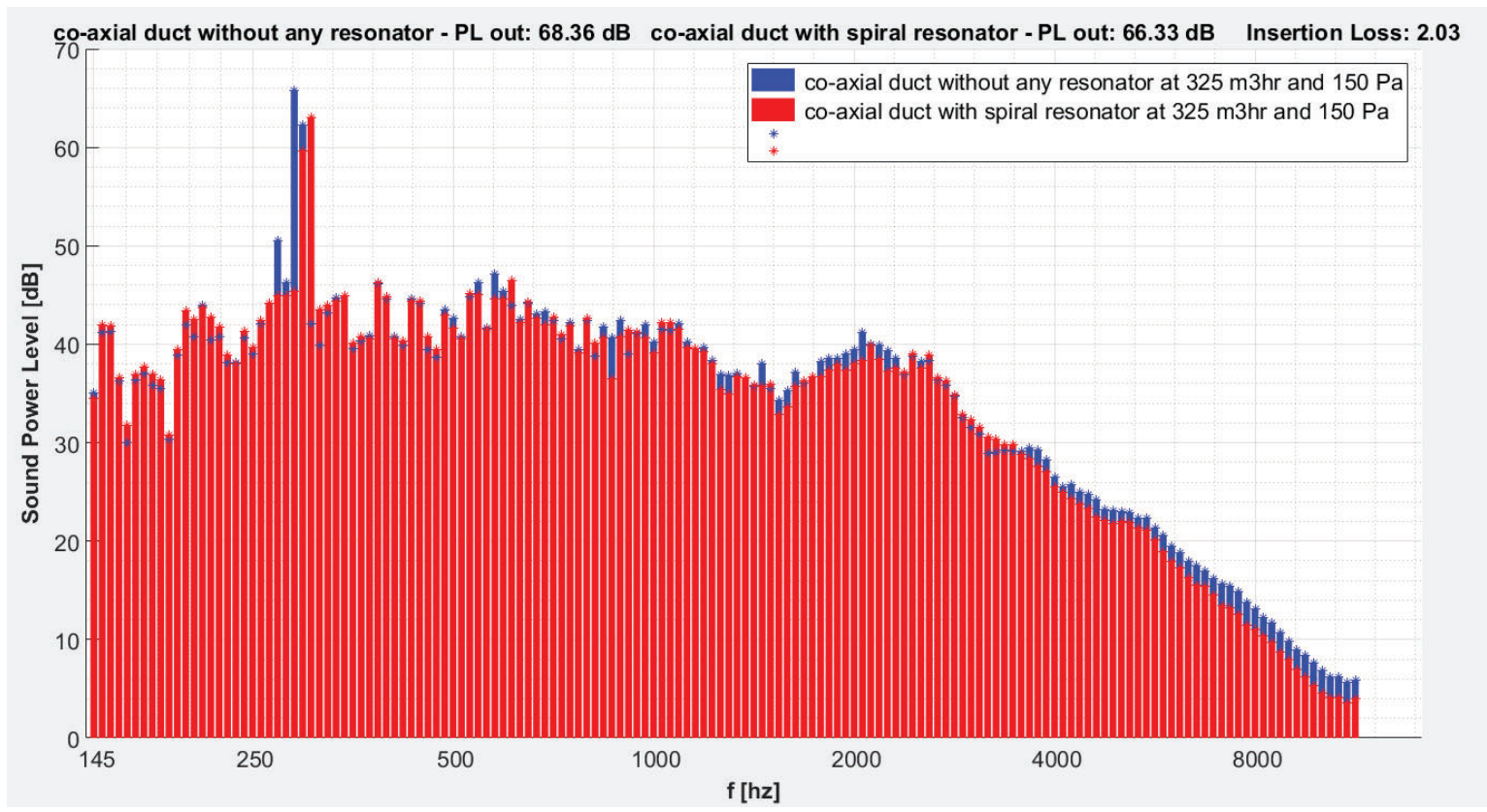

Figure 6.10: Sound power level of applying the spiral resonator at $325 \mathrm{~m}^{3} / \mathrm{h}$ and $150 \mathrm{~Pa}$. 
The insertion loss of the spiral resonator in the real environment is $2.03 \mathrm{~dB}$ but in Fig. 6.10 the eigen-frequencies are not clear, so the first test setup is used to demonstrate the effect of the spiral resonator. The sound power level and the insertion loss for the spiral resonator in the first test setup at $300 \mathrm{~m}^{3} / \mathrm{h}$ flowrate is presented in Fig. 6.11:

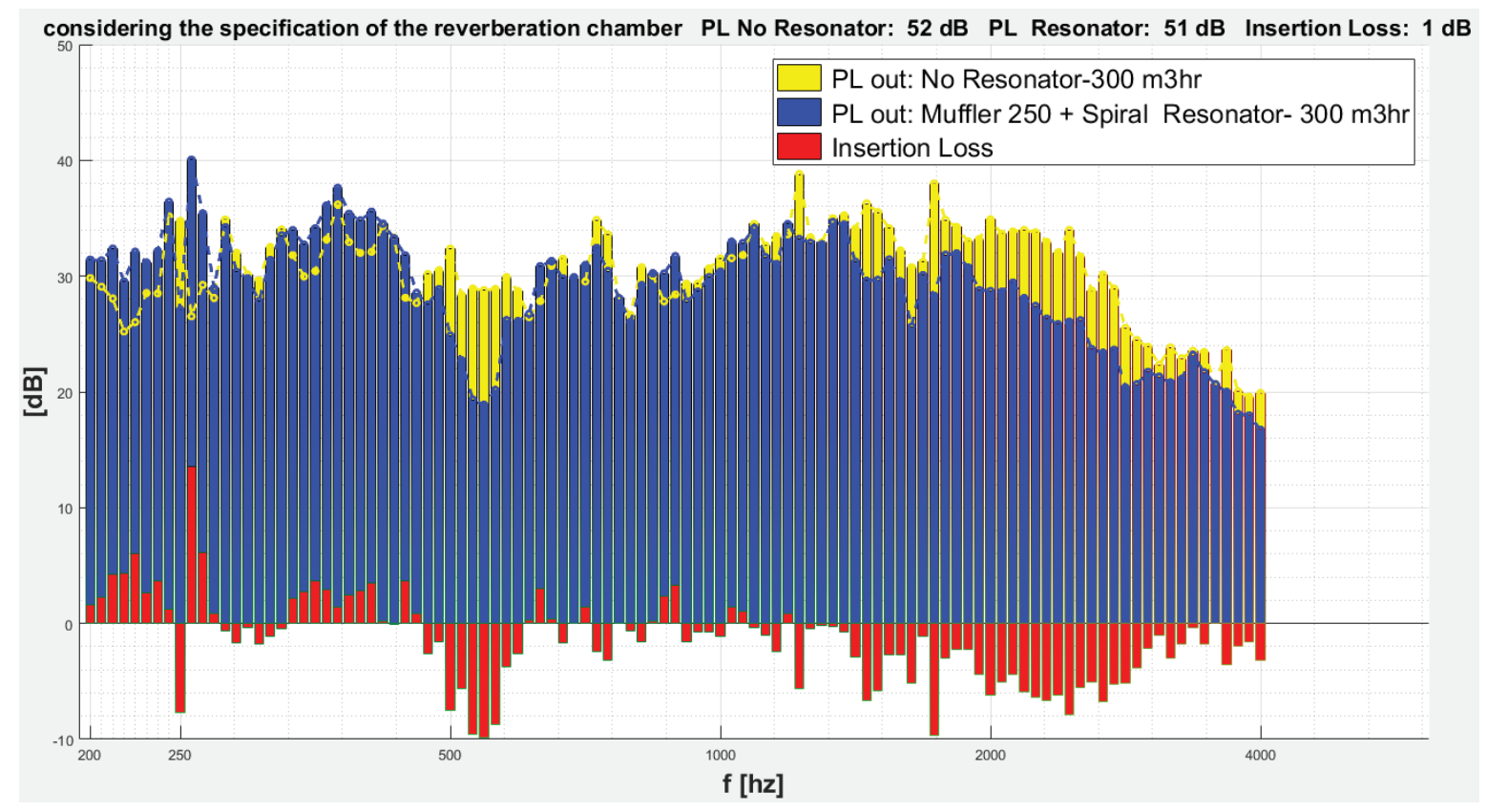

Figure 6.11: Sound power level and Insertion loss of applying the spiral resonator at $300 \mathrm{~m}^{3} / \mathrm{h}$.

As the same to the helicoidal resonator, reducing the noise at the desired eigen-frequencies, i.e., $421 \mathrm{~Hz}, 656 \mathrm{~Hz}, 705 \mathrm{~Hz}, 876 \mathrm{~Hz}$, etc. based on analytical and simulation are achieved however, due to the uncontrolled inlet air pressure, the fan is working harder which causes more noise specially at the critical frequency of the fan, $270 \mathrm{~Hz}$, and the total performance of the resonator becomes lower. 


\subsection{Funnel resonator}

Like the other internal resonators, the funnel resonator shown in Fig. 6.12 is placed inside the co-axial duct before implementing inside the tube.

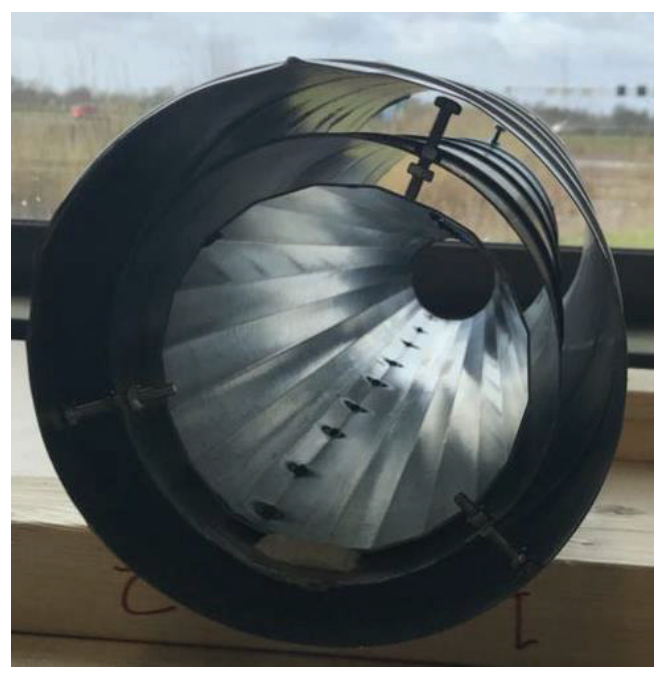

Figure 6.12. The funnel resonator inside the co-axial duct.

The $1 / 24$ octave band sound power level spectrum of the unit by applying the funnel resonator at $325 \mathrm{~m}^{3} / \mathrm{h}$ flowrate and 150 Pa pressure differences between the inlet and outlet ducts is depicted in Fig. 6.13:

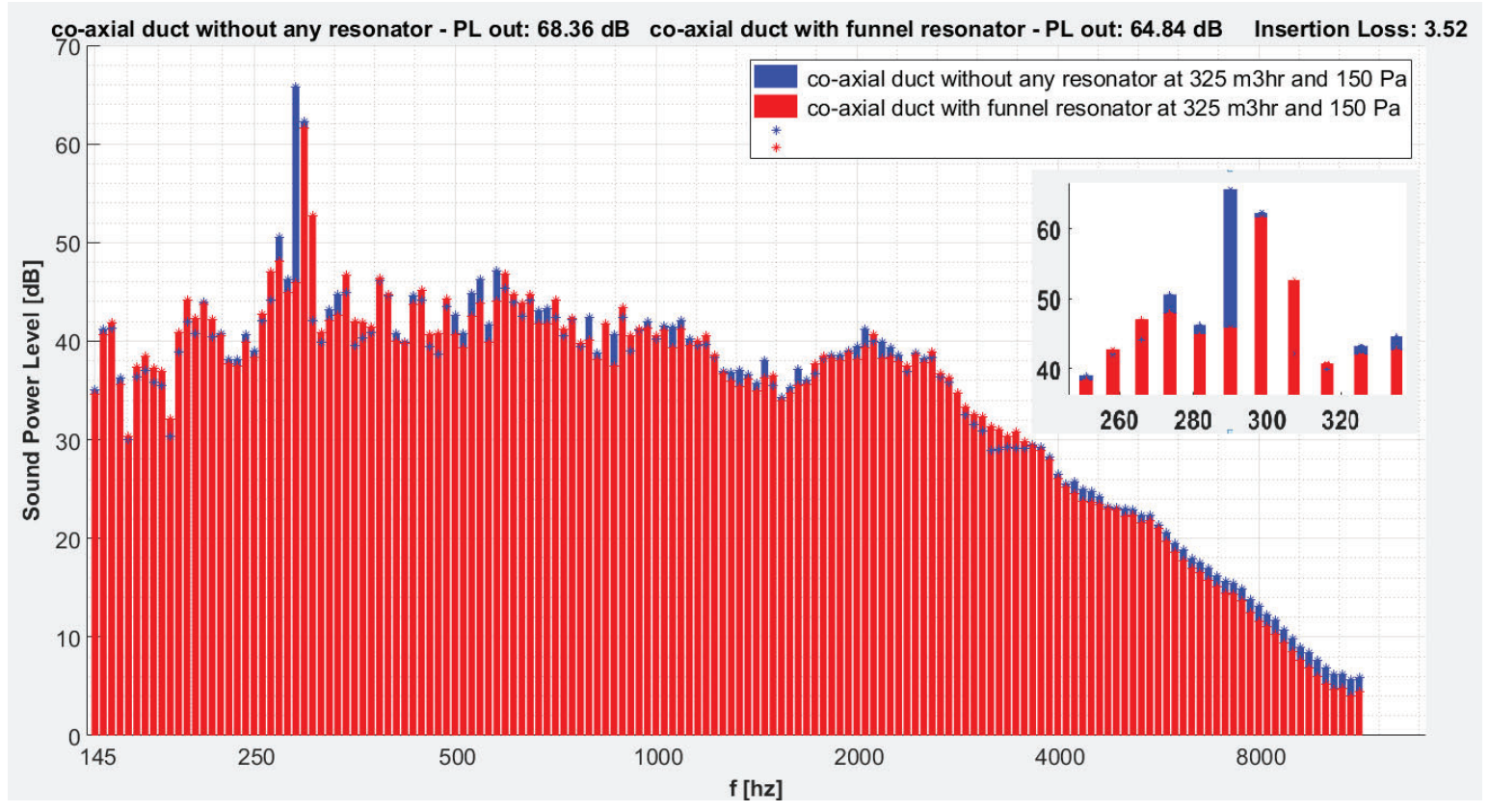

Figure 6.13: Sound power level of applying the funnel resonator at $325 \mathrm{~m}^{3} / \mathrm{h}$ and $150 \mathrm{~Pa}$. 
The measured sound power level at various frequencies is written in Table 6.2 which shows the noise reduction at the desired eigen-frequencies, i.e., $272,540,810 \mathrm{~Hz}$ is achieved.

Table 6.2: measured Sound power level of the unit Flair 325.

\begin{tabular}{|c|c|c|c|c|c|c|c|}
\hline & Lw dB & Lw dB & Lw dB & Lw dB & $L w d B$ & Lw dB & Lwa dB(A) \\
\hline & $250 \mathrm{~Hz}$ & $500 \mathrm{~Hz}$ & $1000 \mathrm{~Hz}$ & $2000 \mathrm{~Hz}$ & $4000 \mathrm{~Hz}$ & $8000 \mathrm{~Hz}$ & overall \\
\hline $\begin{array}{l}\text { co-axial 160mm no silencer } \\
325 \mathrm{~m}^{3} / \mathrm{h}, 150 \mathrm{~Pa}\end{array}$ & 72.1 & 59.6 & 55.9 & 53.7 & 44.7 & 33.1 & 66.2 \\
\hline $\begin{array}{l}\text { co-axial } 160 \mathrm{~mm} \text { funnel } \\
\text { resonator } 325 \mathrm{~m}^{3} / \mathrm{h}, 150 \mathrm{~Pa}\end{array}$ & 67.6 & 59.3 & 55.7 & 53.3 & 45.3 & 32.1 & 63.3 \\
\hline Insertion Loss & 4.5 & 0.3 & 0.2 & 0.4 & -0.6 & 1 & 2.9 \\
\hline
\end{tabular}

\subsection{Acoustic Black Hole muffler}

The first test setup is changed such that the Acoustic Black Hole muffler can be applied at the T-joint which is displayed in Fig. 6.14.
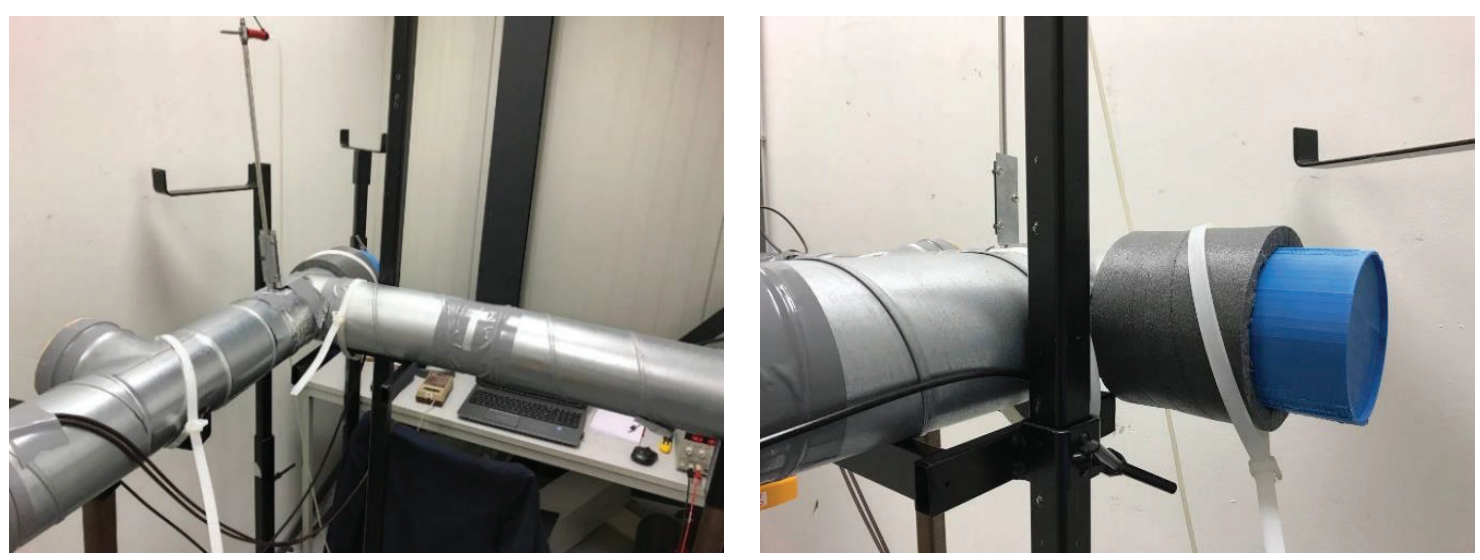

Figure 6.14. Installing the Acoustic Black Hole muffler in the T-joint.

The sound power level and the insertion loss for the Acoustic Black Hole muffler at $300 \mathrm{~m}^{3} / \mathrm{h}$ flowrate is plotted in Fig. 6.15: 


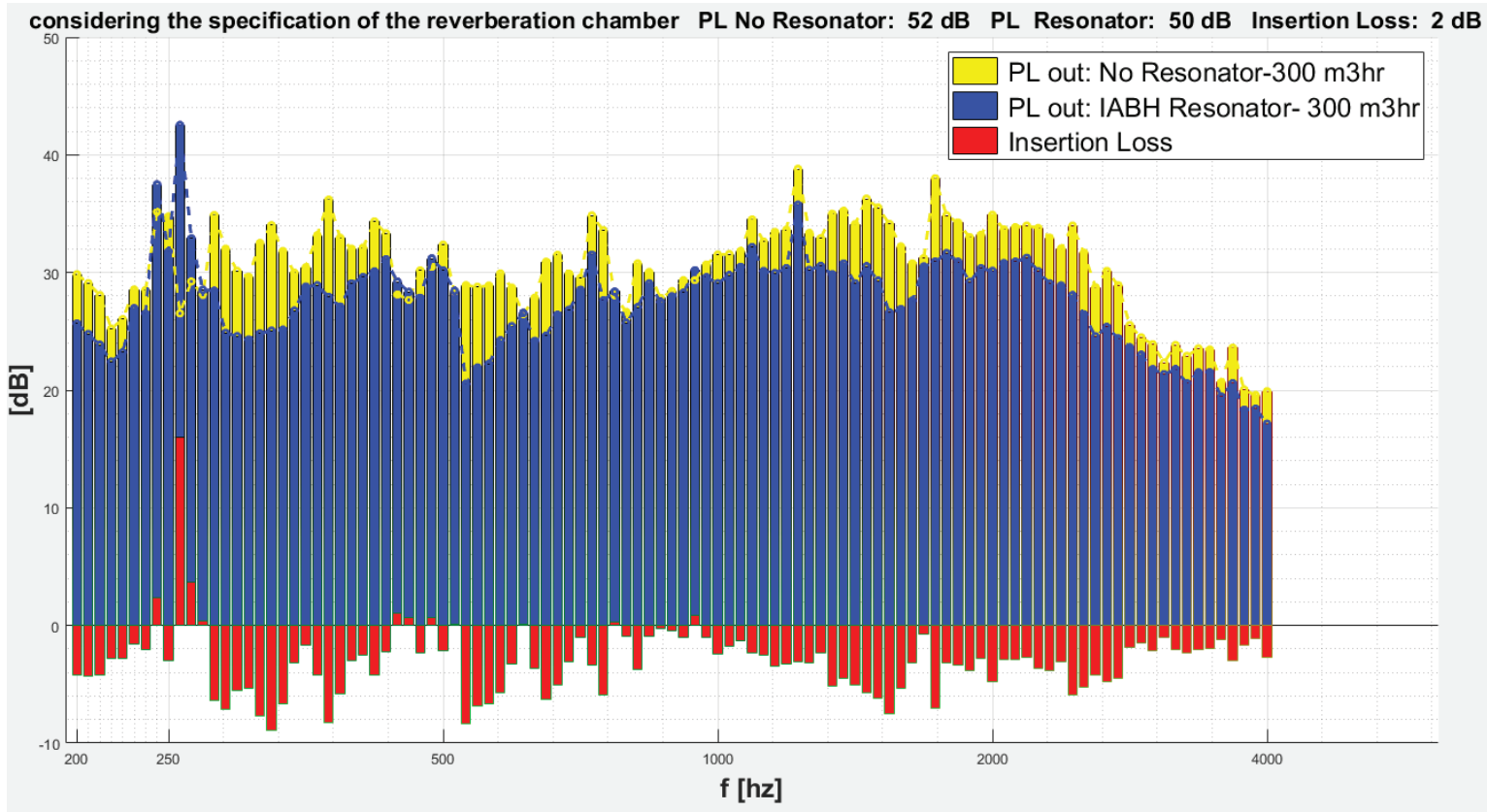

Figure 6.15: Sound power level and Insertion loss of applying the ABH muffler at $300 \mathrm{~m}^{3} / \mathrm{h}$.

As can be seen in Fig. 6.15, the Acoustic Black Hole muffler can be considered as the broadband noise reduction system and it can be installed at each 90 degree elbow to absorb the noise. It should be noted that by modifying the first test setup, the noise at critical frequency will not increase and the total result will be $5 \mathrm{~dB}$ instead of $2 \mathrm{~dB}$.

\subsection{External Acoustic Black Hole muffler}

The first test setup is used to test the external Acoustic Black Hole muffler, shown in Fig. 6.16, in the controlled environment.
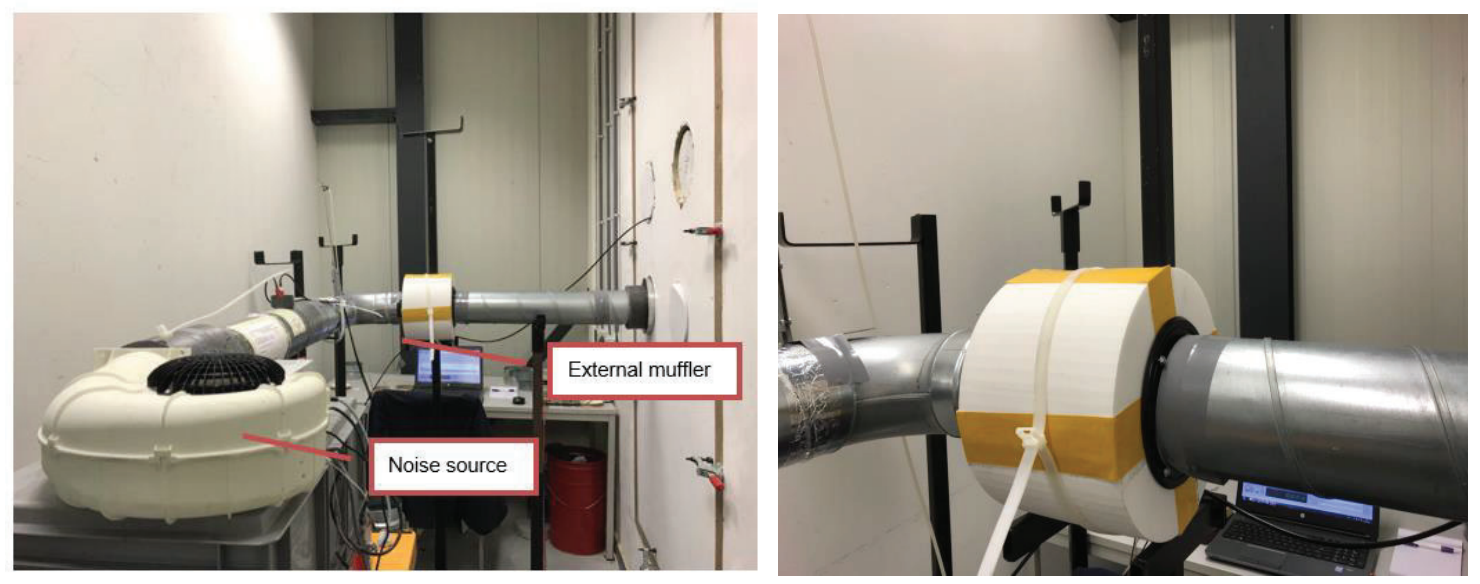

Figure 6.16: The first test setup for testing the external Acoustic Black Hole muffler. 
The sound power level and the insertion loss of the external Acoustic Black Hole muffler at $300 \mathrm{~m}^{3} / \mathrm{h}$ flowrate is plotted in Fig. 6.17 .

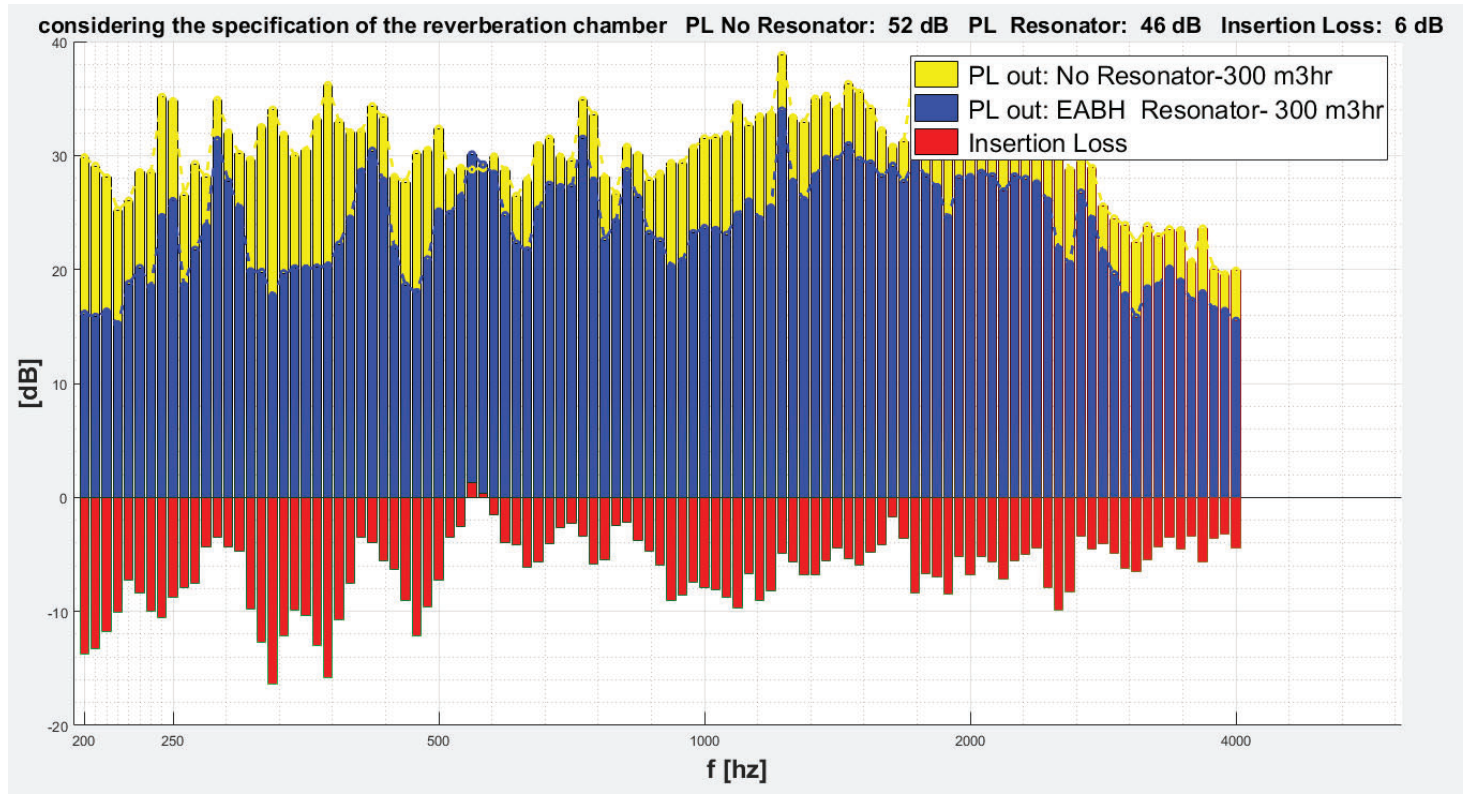

Figure 6.17: Sound power level and Insertion loss of applying the EABH muffler at $300 \mathrm{~m}^{3} / \mathrm{h}$.

The insertion loss of the system is more than $6 \mathrm{~dB}$ which is a big achievement for this project, then the second test setup is prepared to test it in the real environment shown in Fig. 6.18:

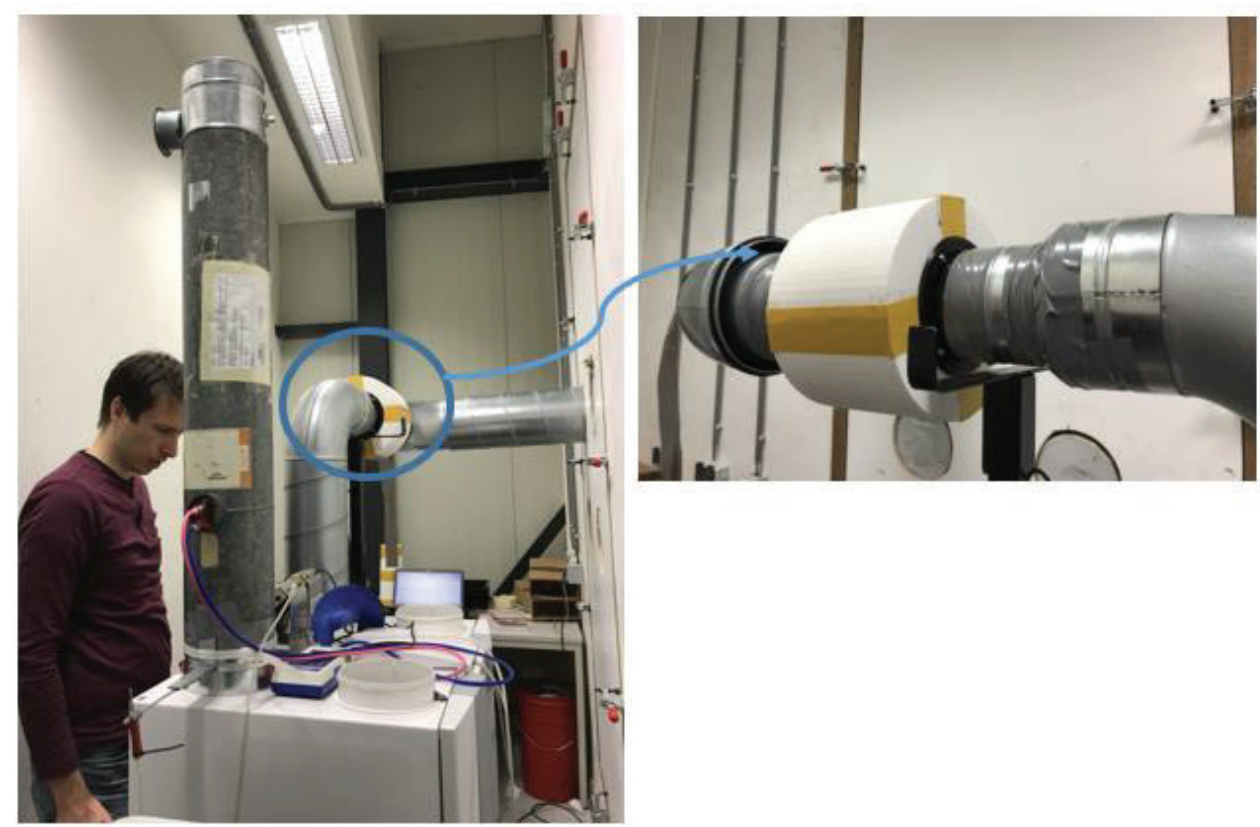

Figure 6.17: The second test setup for testing the EABH muffler in the real environment. 
The sound power level of the external Acoustic Black Hole muffler at $325 \mathrm{~m}^{3} / \mathrm{h}$ flowrate and 150 Pa pressure differences between the inlet and outlet ducts is shown in Fig. 6.18:

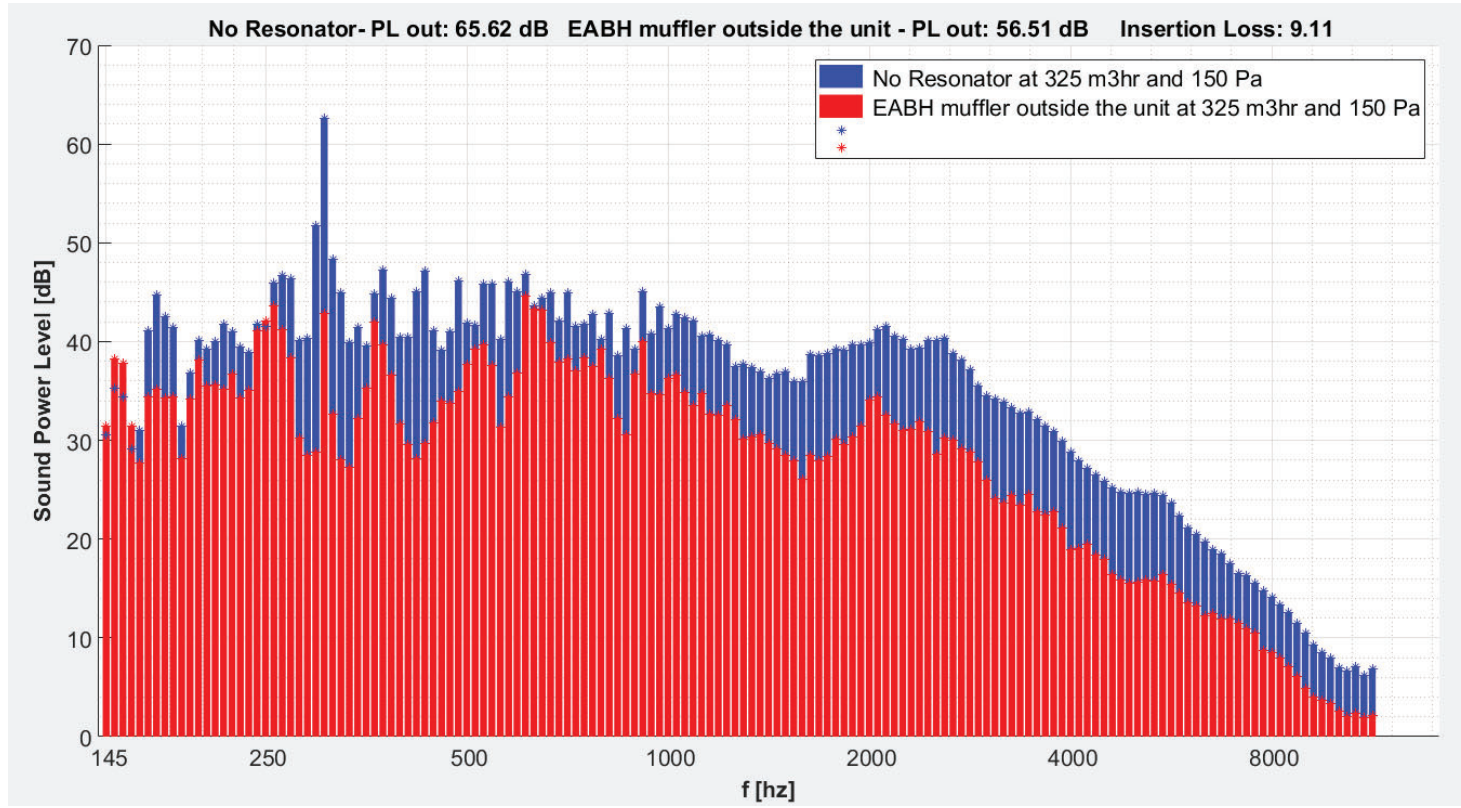

Figure 6.18: PL of applying the EABH muffler at $325 \mathrm{~m}^{3} / \mathrm{h}$ and $150 \mathrm{~Pa}$ in the real environment.

The measured sound power level at various frequencies is written in Table 6.3.

Table 6.3: measured Sound power level of the unit Flair 325.

\begin{tabular}{|c|c|c|c|c|c|c|c|}
\hline & Lw dB & Lw dB & Lw dB & Lw dB & Lw dB & $L w d B$ & Lwa dB(A) \\
\hline & $250 \mathrm{~Hz}$ & $500 \mathrm{~Hz}$ & $1000 \mathrm{~Hz}$ & $2000 \mathrm{~Hz}$ & $4000 \mathrm{~Hz}$ & $8000 \mathrm{~Hz}$ & overall \\
\hline no silencer $325 \mathrm{~m}^{3} / \mathrm{h} 150 \mathrm{~Pa}$ & 69.2 & 60.6 & 56.8 & 54.9 & 47.6 & 34.8 & 64.9 \\
\hline external ABH $325 \mathrm{~m}^{3} / \mathrm{h} 150 \mathrm{~Pa}$ & 57.6 & 54.5 & 51.3 & 46.3 & 38.8 & 27.8 & 56.3 \\
\hline Insertion Loss & 11.6 & 6.1 & 5.5 & 8.6 & 8.8 & 7 & 8.6 \\
\hline
\end{tabular}

The results of Fig. 6.18 and Table. 6.3 reveal that the external Acoustic Black Hole muffler is one of the best mufflers to absorb the broadband noise. To compare the simulation result vs the practical result, the power level and the insertion loss of the system are plotted in Fig. 6.19: 


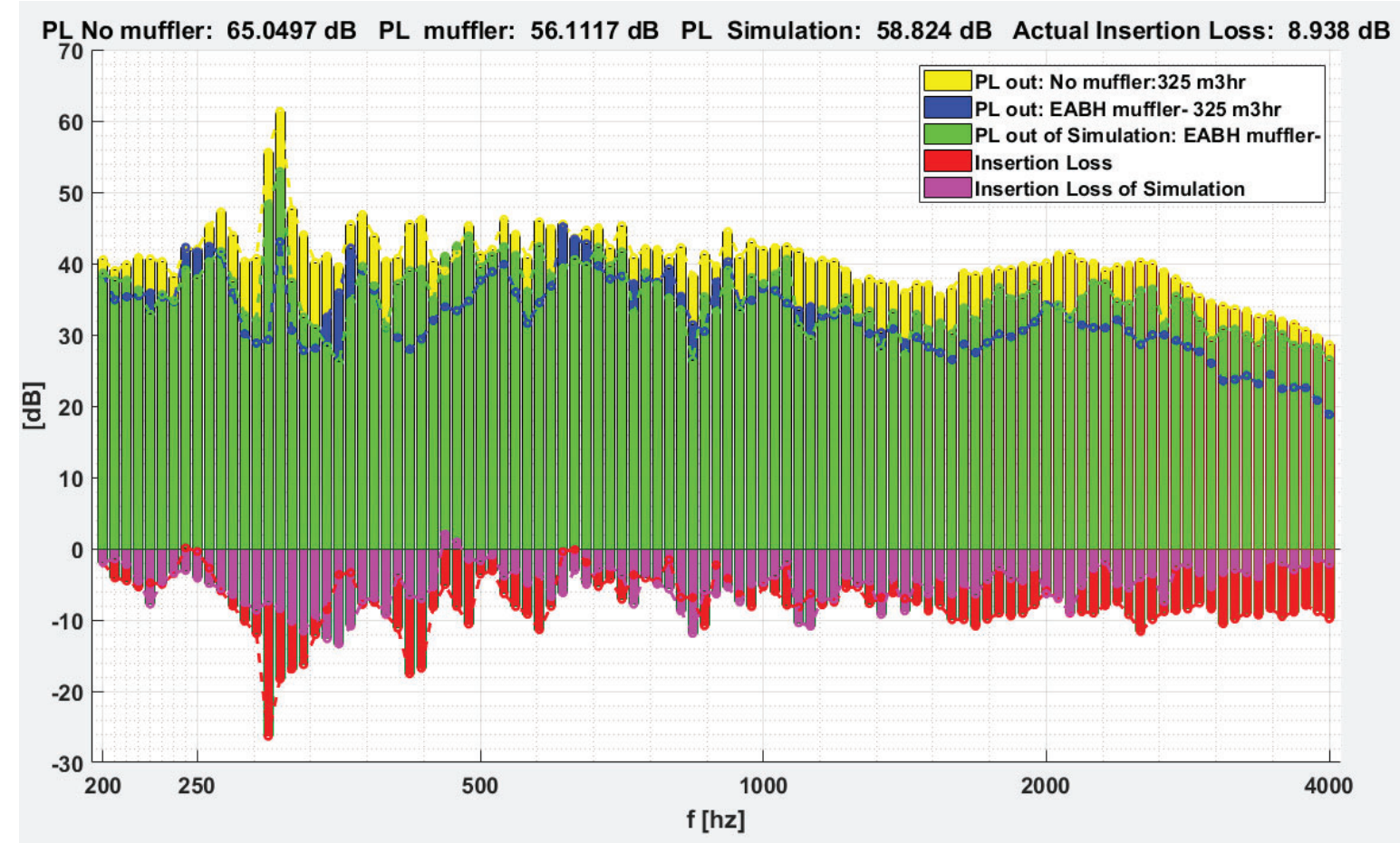

Figure 6.19: PL and IL of applying the EABH muffler at $325 \mathrm{~m}^{3} / \mathrm{h}$ and $150 \mathrm{~Pa}$.

Fig. 6.19 displays a good agreement between the simulation result and practical one however, it could be better by controlling more parameters in the simulation and test.

\subsection{The combination of the External and internal Acoustic Black Hole resonators}

One of the combinations of the mufflers is shown in Fig 6.20, which involves $A B H$ muffler and EABH muffler.
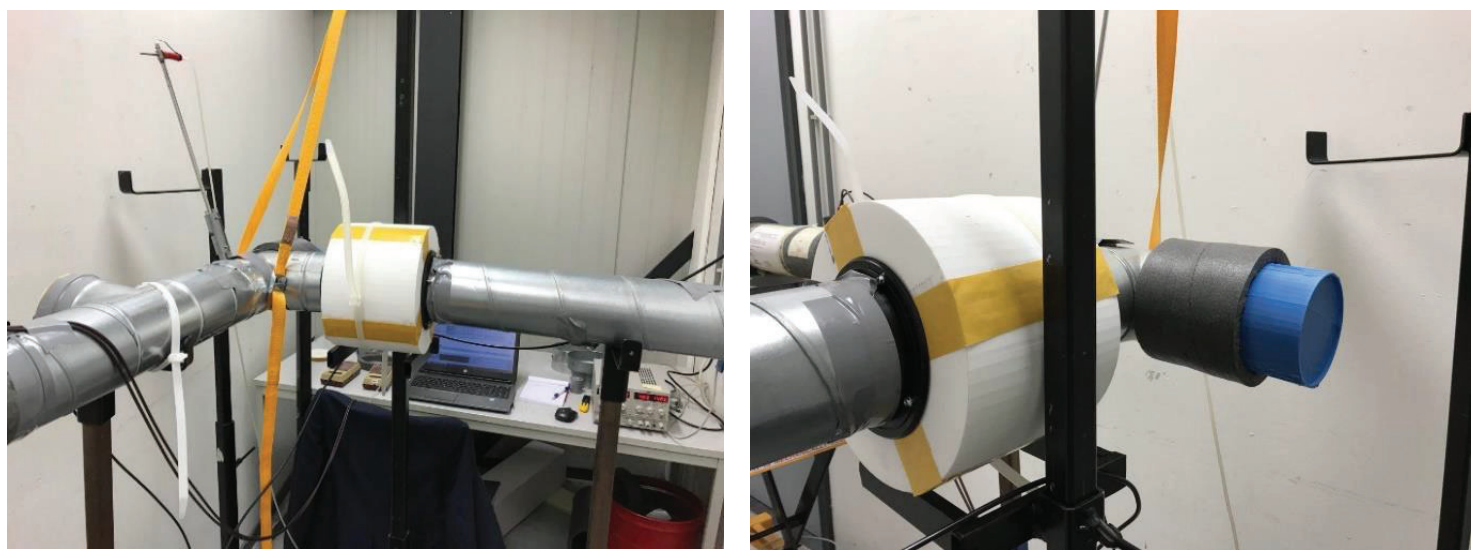

Figure 6.20: Test setup of the combination of the $A B H$ and $E A B H$ mufflers.

The sound power level and the insertion loss of the combination of the $\mathrm{ABH}$ muffler with EABH muffler at the first test setup at $300 \mathrm{~m}^{3} / \mathrm{h}$ flowrate is shown in Fig. 6.21. 


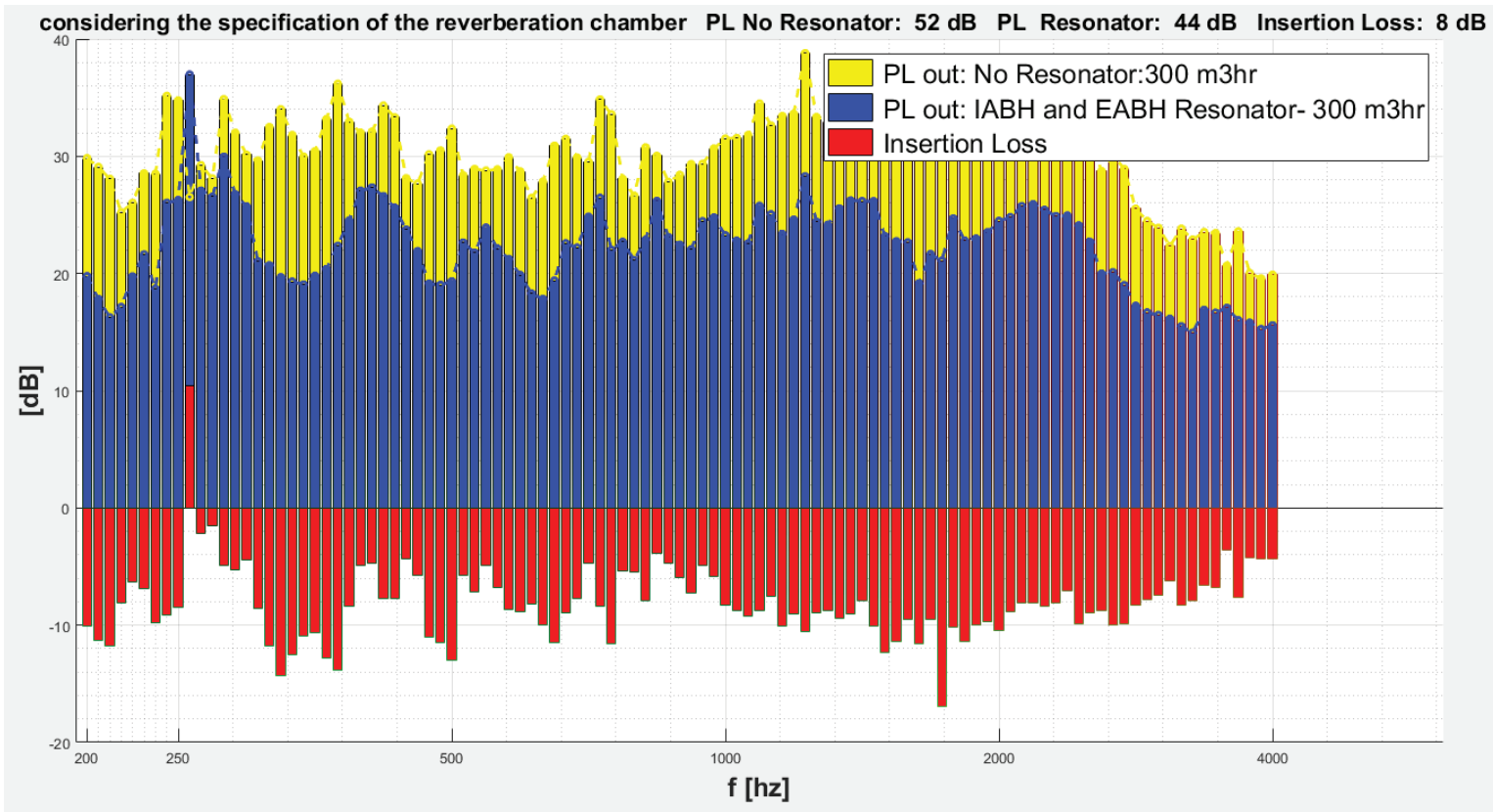

Figure 6.21: Sound power level and Insertion loss of the $A B H$ and EABH mufflers at $300 \mathrm{~m}^{3} / \mathrm{h}$.

\subsection{The internal Acoustic Black Hole muffler}

The second test setup is prepared to test the IABH muffler, put inside the ventilation unit, shown in Fig. 6.22.
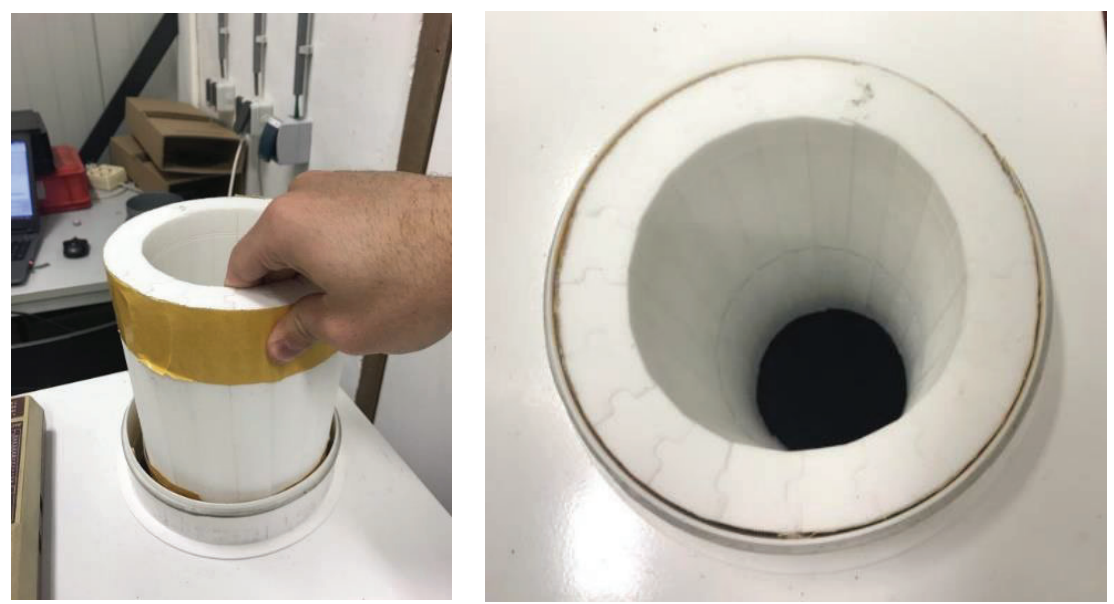

Figure 6.22: The internal Acoustic Black Hole muffler inside the ventilation unit.

The sound power level of the noise measured in the reverberation chamber in two situations, with and without internal Acoustic Black Hole muffler inside the unit, at $325 \mathrm{~m}^{3} / \mathrm{h}$ flowrate and 150 Pa pressure differences between the inlet and outlet ducts is plotted in Fig. 6.23: 


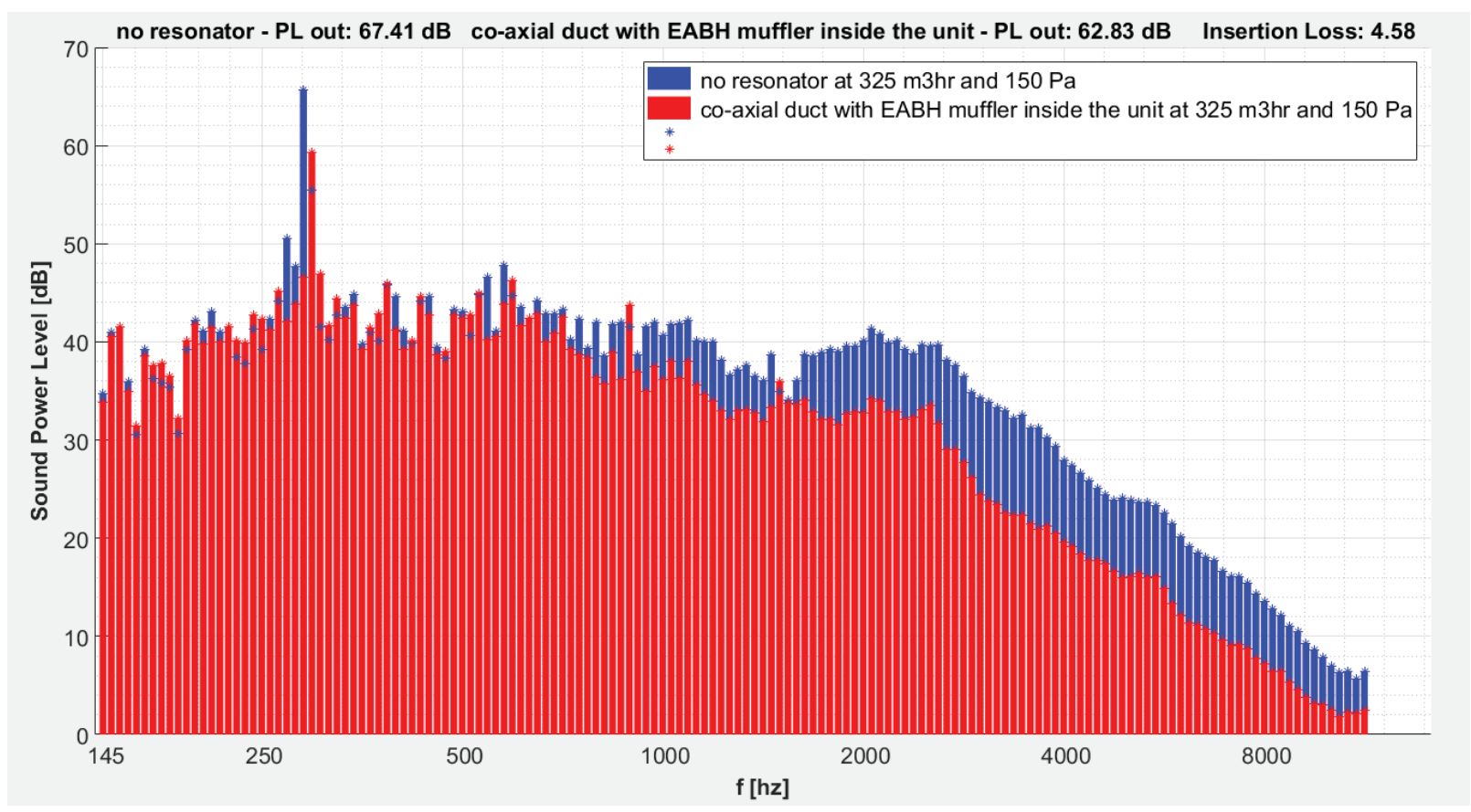

Figure 6.23: Sound power level of the IABH muffler inside the unit at $325 \mathrm{~m}^{3} / \mathrm{h}$ and $150 \mathrm{~Pa}$.

The measured sound power level at various frequencies are depicted in Table 6.4.

Table 6.4: measured Sound power level of the unit Flair 325.

\begin{tabular}{|c|c|c|c|c|c|c|c|}
\hline & Lw dB & Lw dB & Lw dB & Lw dB & Lw dB & Lw dB & Lwa dB(A) \\
\hline & $250 \mathrm{~Hz}$ & $500 \mathrm{~Hz}$ & $1000 \mathrm{~Hz}$ & $2000 \mathrm{~Hz}$ & $4000 \mathrm{~Hz}$ & $8000 \mathrm{~Hz}$ & overall \\
\hline $\begin{array}{l}160 \mathrm{~mm} \text { no silencer } 325 \\
\mathrm{~m}^{3} / \mathrm{h} 150 \mathrm{~Pa}\end{array}$ & 73.1 & 59.5 & 56 & 54.6 & 46.9 & 33.8 & 67 \\
\hline $\begin{array}{l}\text { duct } 160 \mathrm{~mm} \text { internal } \\
\text { ABH } 325 \mathrm{~m}^{3} / \mathrm{h} 150 \mathrm{~Pa}\end{array}$ & 67.2 & 58.5 & 52.8 & 48.5 & 37.8 & 26.1 & 62 \\
\hline Insertion Loss & 5.9 & 1 & 3.2 & 6.1 & 9.1 & 7.7 & 5 \\
\hline
\end{tabular}

In this chapter, 6 prototypes are tested and acoustic performance of each resonator and muffler are obtained. The implementation evaluation of the resonators and mufflers are discussed in the next chapter. 


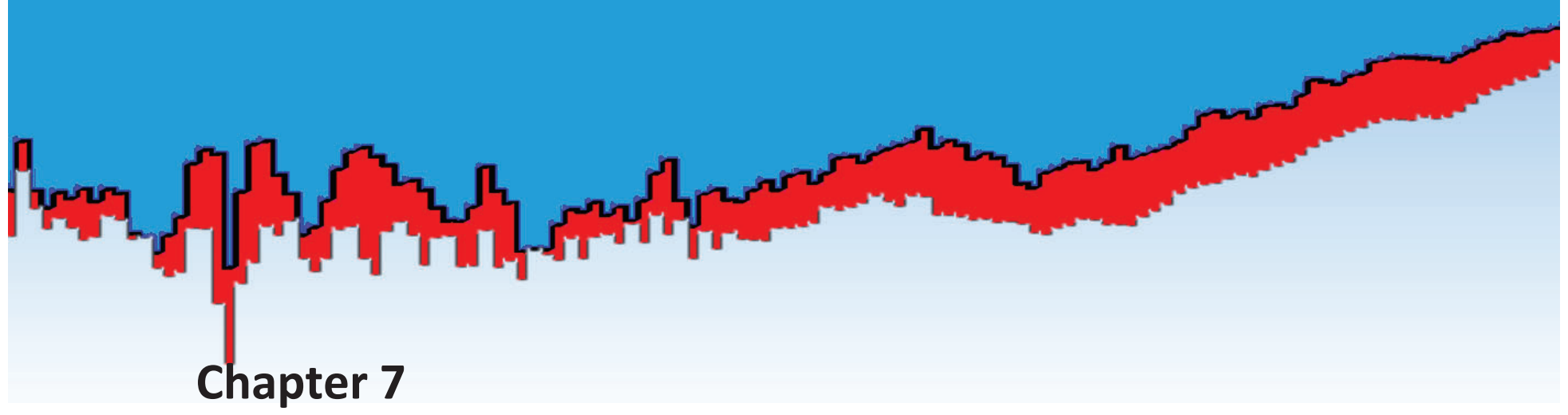

\section{Implementation evaluation and conclusion}

\subsection{Acoustics performance}

To evaluate the acoustical performance of each resonator and muffler, the best TPM value is the insertion loss of the system. The insertion loss of each resonator and muffler is depicted in Table. 7.1:

Table 7.1: measured Insertion Loss of the noise reduction systems.

\begin{tabular}{|l|c|c|c|}
\hline Resonator/muffler & $\begin{array}{l}\text { Flowrate } \\
\mathrm{m3} / \mathrm{h}\end{array}$ & $\begin{array}{l}\text { Pressure difference of inlet } \\
\text { and outlet ducts Pa }\end{array}$ & $\begin{array}{l}\text { Insertion } \\
\text { Loss dB }\end{array}$ \\
\hline Helicoidal resonator & 325 & 150 & 5.37 \\
\hline Spiral resonator & 325 & 150 & 2.03 \\
\hline Funnel resonator & 325 & 150 & 3.52 \\
\hline External Acoustic Black Hole muffler & 325 & 150 & 9.11 \\
\hline Internal Acoustic Black Hole muffler & 325 & 150 & 5.58 \\
\hline
\end{tabular}

\subsection{Pressure drop and energy efficiency}

To define the energy efficiency for each of the noise reduction systems, pressure drop of the resonator or muffler, input power of the ventilation unit and specific power input ratio are suitable TPMs to measure. The results are written in Table. 7.2:

Table 7.2: measured energy TPMs values of the noise reduction systems.

\begin{tabular}{|c|c|c|c|c|c|}
\hline & $\begin{array}{l}\text { Flowrate } \\
\text { m3/h }\end{array}$ & $\begin{array}{l}\text { Pressure } \\
\text { drop over } \\
\text { unit Pa }\end{array}$ & $\begin{array}{l}\text { Pressure drop } \\
\text { over silencer } \\
\text { Pa }\end{array}$ & $\begin{array}{l}\text { Input power } \\
\text { of the unit }\end{array}$ & $\begin{array}{l}\text { Specific } \\
\text { power } \\
\text { input }\end{array}$ \\
\hline No silencer $\mathrm{L}=0.8 \mathrm{~m}$ & 327 & 152 & 12 & $75[\mathrm{~W}]-224[\mathrm{~V}]$ & 0.229 \\
\hline co-axial duct $-160-125 \mathrm{~mm}$ & 326 & 153 & 12 & $75[\mathrm{~W}]-224[\mathrm{~V}]$ & 0.230 \\
\hline Helicoidal resonator & 324 & 153 & 33 & 79 [W]-223 [V] & 0.244 \\
\hline Spiral resonator & 326 & 153 & 24 & $78[\mathrm{~W}]-225[\mathrm{~V}]$ & 0.240 \\
\hline Funnel resonator & 326 & 152 & 27 & 78 [W]-224 [V] & 0.240 \\
\hline External Acoustic Black Hole muffler & 323 & 152 & 22 & $80[\mathrm{~W}]-224[\mathrm{~V}]$ & 0.247 \\
\hline Internal Acoustic Black Hole muffler & 322 & 153 & - & $76[\mathrm{~W}]-226[\mathrm{~V}]$ & 0.236 \\
\hline
\end{tabular}




\subsection{Conclusion}

System engineering guided the structuring of this project. Starting from the problem investigation the design process algorithm was drawn. The next step was treatment design, which involved finding analytical solutions based on solving the wave equation, followed by a knowledge-based preliminary design phase to design the proper treatments. Then, the treatments were validated by numerical simulation. The results guided us to do the next step, comprising the detail designing and development phase. The prototypes were made and tested in controlled and real environments. Finally the treatment implementations were evaluated.

The main outcome of the project is the design and production of six resonators and mufflers, i.e., three internal resonators (helicoidal, spiral, and funnel resonators) to put inside the ventilation ducts, an Acoustic Black Hole muffler that is possible to add in each $90^{\circ}$ elbow by placing it to the T-joint, and two external/internal Acoustic Black Hole mufflers to put outside/inside the ventilation unit. It should be noted that resonators are mainly considered as a narrow band treatments while the mufflers are broadband treatments.

Generally speaking, the analytical and numerical results do match well. Also, the achieved practical eigen-frequencies are almost similar to the derived eigen-frequencies in theory and simulation while the desired performances, IL and PL, are not achieved completely. More time and tests are needed to know how these resonators should be implemented so that the desired noise reduction values will be obtained.

The stakeholder's needs and requirements are met in this project in terms of acoustical performance, space limitations, energy efficiency, and health issues which are explained in detail as follows.

\section{External acoustic black hole muffler}

In terms of the space limitation, the length of the muffler is less than 1 meter $(0.182 \mathrm{~m})$, the outer diameter of the external muffler is $254 \mathrm{~mm}$ which is less than $300 \mathrm{~mm}$, and the inner diameter is similar to the normal duct size of $125 \mathrm{~mm}$. The insertion loss of the system is 9.1 $\mathrm{dB}$ which is near to the desired $10 \mathrm{~dB}$. However, by combining three of these mufflers, the insertion loss will be more than $20 \mathrm{~dB}$. Also by increasing the length of the muffler the transmission loss at lower frequencies can be increased. In terms of energy efficiency, this 
muffler is installed surrounding the pipe and generally it does not have any pressure drop, but due to the connections needed in this model the input power of the fan is increased from $75 \mathrm{~W}$ to $80 \mathrm{~W}$. Moreover, this treatment is a passive noise reduction technique. Possible health issues can be prevented by adding a membrane at the inner side of the muffler so that air cannot flow directly into the muffler. Hence the health issue regarding growing the bacteria inside the muffler will not happen.

\section{Internal acoustic black hole muffler}

In terms of the space limitation, the muffler is fitted exactly inside the diffuser of the ventilation system (model: Flair 325). The insertion loss of the system is $5.58 \mathrm{~dB}$ which is more than expected in simulation, $2.3 \mathrm{~dB}$. It may result from the sensitivity of the system to the pressure differences between the inlet and outlet ducts of the unit, i.e., by increasing even 1 Pa more, the noise level at the critical frequency will increase extremely. In terms of energy efficiency, this muffler is installed inside the unit and it produces more pressure drop over the unit; the input power of the fan is increased from $75 \mathrm{~W}$ to $76 \mathrm{~W}$. In terms of health issues, this resonator is similar to the EABH muffler and it does not have any problem in this regard.

\section{Helicoidal resonator}

In terms of the space limitation, it is installed inside a duct with a diameter more than $125 \mathrm{~mm}$ and it does not have any problem, however it could be possible to incorporate it inside the unit due to the length of the resonator being less than $35 \mathrm{~cm}$. The insertion loss of the system is $5.37 \mathrm{~dB}$ which is a good achievement for this type of the resonator, since it can only tune the noise at specific frequency (more than $1180 \mathrm{~Hz}$ ). In terms of energy efficiency, this muffler should be installed inside the co-axial duct to not increase the pressure drop over the resonator dramatically which causes the production of more noise from the fan. In case of installation inside a co-axial duct, the input power of the unit is increased from $75 \mathrm{~W}$ to $79 \mathrm{~W}$. Also, this treatment like the previous treatments does not have health issues.

\section{Spiral resonator}

In terms of the space limitation, it is installed inside ducts, whereas the length and number of turns give more opportunity to tune the noise at desired frequencies. The insertion loss of the system is $2 \mathrm{~dB}$, which means that more time is needed to find out how it should be implemented to get the desired result. It is obvious that this resonator is much more effective than a helicoidal resonator at low frequencies. In terms of energy efficiency, this muffler 
should be installed inside the co-axial duct. The input power of the unit is increased from 75 $\mathrm{W}$ to $78 \mathrm{~W}$. Also, this treatment like the previous treatments does not have health issues.

\section{Funnel resonator}

In terms of the space limitation, it should be installed inside the co-axial duct. The insertion loss of the system is $3.52 \mathrm{~dB}$ which mainly happens at the designed frequency, $287 \mathrm{~Hz}$. In terms of energy efficiency, the input power of the unit is increased from $75 \mathrm{~W}$ to $78 \mathrm{~W}$. Moreover, this treatment like the previous treatments does not have health issues.

An additional solution could be obtained by inserting the spiral-helicoidal resonator and funnel-spiral-helicoidal resonator inside the duct, which makes it possible to absorb the noise at any desired low frequency. 


\subsection{Future work}

For various types of the ventilation units at Brink Climate Systems, one or a combination of these developed resonators and mufflers could be the best choice due to the use limitations, advantages and disadvantages of each method. Therefore, developing an analysis and design tool to support the designer in choosing the right methodology for the problem at hand could be the next project for the near future.

By analyzing the noise spectrum of the unit (see Fig. 6.6) it can be seen that the critical frequency is between 250 to $300 \mathrm{~Hz}$. One of the main reasons for high noise power level at this frequency range is a propeller flowmeter installed inside the fan shown in Fig. 7.1. The fan optimization was not considered in this project, but it could be considered to change this type of flowmeter with another one. For instance, a new invented acoustical flowmeter or ultrasonic flowmeter could be good solutions in this respect.

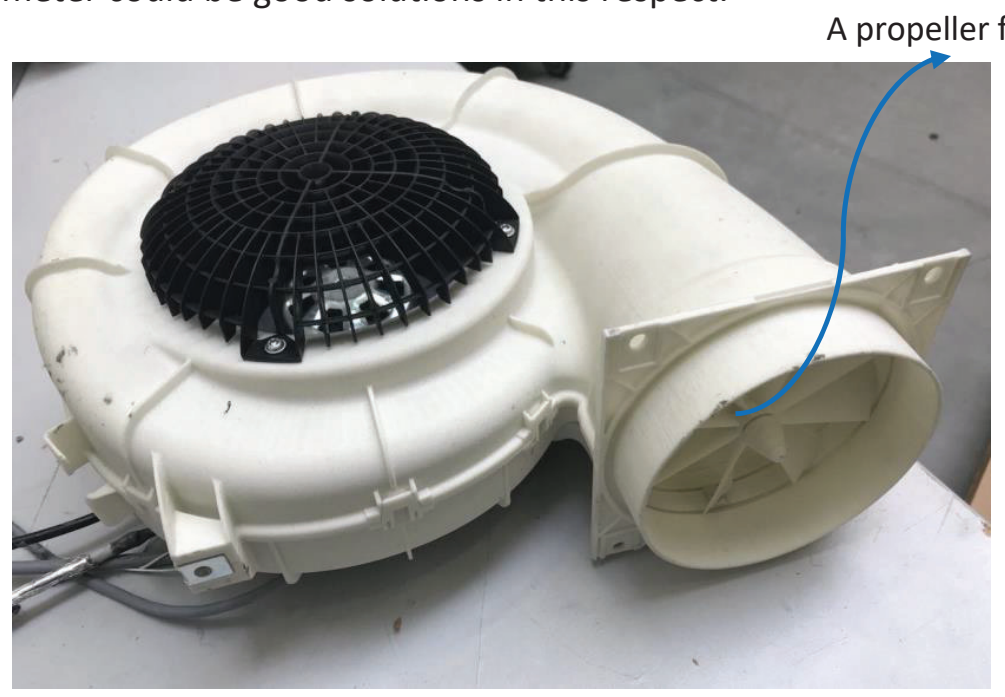

Figure 7.1: The fan of the ventilation unit with a propeller flowmeter inside.

To get good correlation between analytical, numerical, and practical results, it is needed to consider more parameters in equations and simulations. Somehow, the simulated models become more similar to the system in the real environment. Analyzing this type of the complicated models takes more time and energy, so it could be another future topic.

Finally, an improvement of each of the mentioned resonators and mufflers could be undertaken to get still better acoustical performance and energy efficiency. 


\section{Appendix A.}

- The pressure gradient in cylindrical coordinate could be written as:

$$
\begin{aligned}
& \nabla \vec{P}=\frac{\partial R}{\partial r} \vec{e}_{r}+\frac{1}{r} \frac{\partial \Phi}{\partial \varphi} \vec{e}_{\varphi}+\frac{\partial Z}{\partial z} \vec{e}_{z}, \vec{e}_{r}=\left(\begin{array}{c}
\cos (\varphi) \\
\sin (\varphi) \\
0
\end{array}\right), \vec{e}_{\varphi}=\left(\begin{array}{c}
-\sin (\varphi) \\
\cos (\varphi) \\
0
\end{array}\right), \vec{e}_{z}=\left(\begin{array}{l}
0 \\
0 \\
1
\end{array}\right) \\
& \Rightarrow \nabla \vec{P}=\left(\begin{array}{l}
\frac{\partial R}{\partial r} \cos (\varphi)-\frac{1}{r} \frac{\partial \Phi}{\partial \varphi} \sin (\varphi) \\
\frac{\partial R}{\partial r} \sin (\varphi)+\frac{1}{r} \frac{\partial \Phi}{\partial \varphi} \cos (\varphi) \\
\frac{\partial Z}{\partial z}
\end{array}\right)
\end{aligned}
$$

The helicoid surface could be expressed:

$$
\text { Helicoid Surface }=\left(\begin{array}{c}
r \cos \left(\frac{2 \pi}{L} z\right) \\
r \sin \left(\frac{2 \pi}{L} z\right) \\
z
\end{array}\right), \varphi=\frac{2 \pi}{L} z .
$$

To find the normal vector of the surface, the derivation with respect to the $r$ and $z$ is needed:

$$
\vec{S}_{r}=\left(\begin{array}{c}
\cos \left(\frac{2 \pi}{L} z\right) \\
\sin \left(\frac{2 \pi}{L} z\right) \\
0
\end{array}\right), \vec{S}_{z}=\left(\begin{array}{c}
-\frac{2 \pi r}{L} \sin \left(\frac{2 \pi}{L} z\right) \\
\frac{2 \pi r}{L} \cos \left(\frac{2 \pi}{L} z\right) \\
1
\end{array}\right)
$$

The normal vector of the surface could be found by the cross product of the above vectors:

$$
\hat{n}=\vec{S}_{r} \times \vec{S}_{z}=\left(\begin{array}{c}
\sin \left(\frac{2 \pi}{L} z\right) \\
-\cos \left(\frac{2 \pi}{L} z\right) \\
\frac{2 \pi r}{L}
\end{array}\right) .
$$


- Function and normal vector of the spiral surface could be expressed:

$$
\begin{gathered}
\text { Spiral Surface }=\left(\begin{array}{c}
\frac{r_{2} \varphi}{2 n \pi} \cos (\varphi) \\
\frac{r_{2} \varphi}{2 n \pi} \sin (\varphi) \\
z
\end{array}\right) \vec{S}_{\varphi}=\left(\begin{array}{c}
\frac{r_{2}}{2 n \pi} \cos (\varphi)-\frac{r_{2} \varphi}{2 n \pi} \sin (\varphi) \\
\frac{r_{2}}{2 n \pi} \sin (\varphi)+\frac{r_{2} \varphi}{2 n \pi} \cos (\varphi) \\
0
\end{array}\right), \vec{S}_{z}=\left(\begin{array}{l}
0 \\
0 \\
1
\end{array}\right) \\
\hat{n}=\vec{S}_{\varphi} \times \vec{S}_{z}=\left(\begin{array}{c}
\frac{r_{2}}{2 n \pi} \sin (\varphi)+\frac{r_{2} \varphi}{2 n \pi} \cos (\varphi) \\
-\frac{r_{2}}{2 n \pi} \cos (\varphi)+\frac{r_{2} \varphi}{2 n \pi} \sin (\varphi) \\
0
\end{array}\right) .
\end{gathered}
$$

- For the regions, 2 and 3, in spiral resonator (see Fig. 3.8) we have:

$$
\begin{gathered}
\left\{\begin{array}{c}
A_{m 1} J_{m}^{\prime}\left(k_{r} r_{1}\right)=\left[A_{m 2} J_{m}^{\prime}\left(k_{r} r_{1}\right)+B_{m 2} Y_{m}^{\prime}\left(k_{r} r_{1}\right)\right] \\
r_{1} \varphi_{1} A_{m 1} J_{m}^{\prime}\left(k_{r} r_{1}\right)=r_{2} \varphi_{2}\left[A_{m 2} J_{m}^{\prime}\left(k_{r} r_{2}\right)+B_{m 2} Y_{m}^{\prime}\left(k_{r} r_{2}\right)\right]
\end{array} \Rightarrow\right. \\
\left\{\begin{array}{c}
A_{m 2}(r)=A_{m 1}(r) *\left[\frac{r_{1} \varphi_{1} J_{m}^{\prime}\left(k_{r} r_{1}\right)}{r_{2} \varphi_{2} J_{m}^{\prime}\left(k_{r} r_{2}\right)}-\frac{J_{m}^{\prime}\left(k_{r} r_{1}\right)}{Y_{m}^{\prime}\left(k_{r} r_{1}\right)} \frac{Y_{m}^{\prime}\left(k_{r} r_{2}\right)}{J_{m}^{\prime}\left(k_{r} r_{2}\right)}\right] /\left[1-\frac{J_{m}^{\prime}\left(k_{r} r_{1}\right)}{Y_{m}^{\prime}\left(k_{r} r_{1}\right)} \frac{Y_{m}^{\prime}\left(k_{r} r_{2}\right)}{J_{m}^{\prime}\left(k_{r} r_{2}\right)}\right] \\
B_{m 2}(r)=\left[A_{m 1}(r)-A_{m 2}(r)\right] * \frac{J_{m}^{\prime}\left(k_{r} r_{1}\right)}{Y_{m}^{\prime}\left(k_{r} r_{1}\right)}
\end{array}\right.
\end{gathered}
$$

For the last region, Re. 4, unknown parameters will be derived as:

$$
\begin{gathered}
\left\{\begin{array}{c}
r_{1} \varphi_{1} A_{m 1} J_{m}^{\prime}\left(k_{r} r_{1}\right)=r_{2} \varphi_{2}\left[A_{m 3} J_{m}^{\prime}\left(k_{r} r_{2}\right)+B_{m 3} Y_{m}^{\prime}\left(k_{r} r_{2}\right)\right] \\
A_{m 3} J_{m}^{\prime}\left(k_{r} r_{3}\right)+B_{m 3} Y_{m}^{\prime}\left(k_{r} r_{3}\right)=0
\end{array} \Rightarrow\right. \\
\left\{\begin{array}{c}
A_{m 3}(r)=A_{m 1}(r) *\left[\frac{r_{1} \varphi_{1}}{r_{2} \varphi_{2}} J_{m}^{\prime}\left(k_{r} r_{1}\right)\right] /\left[J_{m}^{\prime}\left(k_{r} r_{2}\right)-\frac{J_{m}^{\prime}\left(k_{r} r_{3}\right)}{Y_{m}^{\prime}\left(k_{r} r_{3}\right)} Y_{m}^{\prime}\left(k_{r} r_{2}\right)\right] . \\
B_{m 3}(r)=-A_{m 3}(r) * \frac{J_{m}^{\prime}\left(k_{r} r_{3}\right)}{Y_{m}^{\prime}\left(k_{r} r_{3}\right)}
\end{array}\right.
\end{gathered}
$$

- The funnel surface can be expressed:

$$
\text { Funnel Surface }=\left(\begin{array}{c}
\left(r_{1}+\frac{r_{2}-r_{1}}{L} z\right) \cos (\varphi) \\
\left(r_{1}+\frac{r_{2}-r_{1}}{L} z\right) \sin (\varphi) \\
z
\end{array}\right)
$$

To find the normal vector of the funnel surface, the derivation respect to the $r$ and $z$ is needed: 


$$
\vec{S}_{\phi}=\left(\begin{array}{c}
-\left(r_{1}+\frac{r_{2}-r_{1}}{L} z\right) \sin (\phi) \\
\left(r_{1}+\frac{r_{2}-r_{1}}{L} z\right) \cos (\phi) \\
0
\end{array}\right), \vec{S}_{f}=\left(\begin{array}{c}
\frac{r_{2}-r_{1}}{L} \operatorname{Cos}(\phi) \\
\frac{r_{2}-r_{1}}{L} \operatorname{Sin}(\phi) \\
1
\end{array}\right) * \frac{L}{r_{2}-r_{1}}
$$

The normal vector of the surface can be found by the cross product of the above vectors:

$$
\hat{n}=\vec{S}_{\phi} \times \vec{S}_{z}=\left(\begin{array}{c}
\left(\frac{L}{r_{2}-r_{1}} r_{1}+z\right) \cos (\phi) \\
\left(\frac{L}{r_{2}-r_{1}} r_{1}+z\right) \sin (\phi) \\
-\left(\frac{L}{r_{2}-r_{1}} r_{1}+z\right)
\end{array}\right) .
$$

- The approximate of the first and second kinds of Bessel functions are:

$$
J_{m}(x)=\left\{\begin{array} { l l l } 
{ \sum _ { l = 0 } ^ { \infty } \frac { ( - 1 ) ^ { l } } { 2 ^ { 2 l + | m | } l ! ( | m | + l ) ! } x ^ { 2 l + | m | } } & { \text { for } | m | \neq \frac { 1 } { 2 } } \\
{ \sqrt { \frac { 2 } { \pi x } \operatorname { c o s } x } } & { \text { for } m = - \frac { 1 } { 2 } }
\end{array} \quad Y _ { m } ( x ) \sim \left\{\begin{array}{ll}
\frac{2}{\pi}\left[\ln \left(\frac{1}{2} x\right)+\gamma\right] & m=0, x \ll 1 \\
-\frac{\Gamma(m)}{\pi}\left(\frac{2}{x}\right)^{m} & m \neq 0, x \ll 1
\end{array}\right.\right.
$$

Based on the approximate of the first and second kinds of Bessel functions, the Taylor's expansions for $m=0$ are:

$$
\begin{aligned}
& J_{0}(x)=1-\frac{1}{2^{2}} x^{2}+\frac{1}{2^{4}(2 !)^{2}} x^{4}-\ldots \\
& J_{0}^{\prime}(x)=-\frac{2}{2^{2}} x+\frac{4}{2^{4}(2 !)^{2}} x^{3}-\ldots \\
& Y_{0}(x)=\left\{\begin{array}{l}
\frac{2}{\pi}\left[\operatorname{Ln}\left(\frac{x}{2}\right)+0.57721\right] x<<1 \\
\sqrt{\frac{2}{\pi x}} \operatorname{Sin}\left(x-\frac{\pi}{4}\right) x>>1
\end{array}\right. \\
& Y_{0}^{\prime}(x)=\left\{\begin{array}{l}
\frac{1}{x \pi} \quad x<<1 \\
\sqrt{\frac{2}{\pi x}} \operatorname{Cos}\left(x-\frac{\pi}{4}\right)-\sqrt{\frac{2}{\pi}} \frac{1}{2 x^{1.5}} \operatorname{Sin}\left(x-\frac{\pi}{4}\right) x>>1
\end{array}\right.
\end{aligned}
$$




\section{References}

[1] Brink, “About Brink," 2018. [Online]. Available:

https://www.brinkclimatesystems.nl/en-us/international/home.

[2] R. Wieringa, Design Science Methodology for Information Systems and Software Engineering. 2014.

[3] K. Visscher and J. I. A. Visscher-Voerman, "Organizational design approaches in management consulting," Manag. Decis., vol. 48, no. 5, pp. 713-731, 2010.

[4] M. Kojourimanesh, "Literature Study for PDEng- Noise reduction for ventilation systems with heat exchangers," 2016.

[5] G. M. Bonnema, T. Veenvliet, and J. F. Broenink, systems design and engineering. 2013.

[6] Brink, Installation \& Regulations of Flair 325. 2017.

[7] W. O. Schotborgh, C. Mcmahon, and F. J. A. M. Van Houten, "A knowledge acquisition method to model parametric engineering design processes," vol. 4, 2012.

[8] J. M. J. Becker and W. W. Wits, "A Template for Design for eXcellence (DfX) Methods," Smart Prod. Eng., vol. 5, pp. 33-42, 2013.

[9] W. Lapka, "Numerical study of acoustic-structure interaction of selected helicoidal resonator with flexible helicoidal profile," 2016.

[10] M. Schroeder, Springer Handbook of Acoustics. 2007.

[11] L. E. Kinsler, A. R. Frey, A. . Coppens, and J. V. Sanders, Fundamentals of Acoustics. New York: John Wiley \&Sons, Inc, 2000.

[12] ISO 3741: Preview Acoustics -- Determination of sound power levels and sound energy levels of noise sources using sound pressure -- Precision methods for reverberation test rooms. 2010.

[13] M. L. Munjal, Acoustics of Ducts and Mufflers. John Wiley \&Sons, Inc, 1987.

[14] S. Errede, "Mathematical Musical Physics of the Wave Equation - Part 2," pp. 1-26, 2017.

[15] F. Jacobsen, "Propagation of sound waves in ducts," Lect. notes, no. 31260, p. 71, 2011.

[16] Comsol, “Comsol Multiphysics," User Guid., pp. 630-645, 2012.

[17] A. Bayliss, M. Gunzburger, and E. Turkel, "Boundary Conditions for the Numerical Solution of Elliptic Equations in Exterior Regions," SIAM J. Appl. Math., vol. 42, no. 2, pp. 430-451, 1982.

[18] D. Givoli and B. Neta, "High-order non-reflecting boundary scheme for time-dependent waves," J. Comput. Phys., vol. 186, no. 1, pp. 24-26, 2003.

[19] M. A. Mironov and V. V Pislyakov, "One-Dimensional Acoustic Waves in Retarding Structures with Propagation Velocity Tending to Zero," Acoustical Physics, vol. 48, no. 3, pp. 347-352, 2002. 
[20] S. Marburg and H. J. Hardtke, "Study on the acoustic boundary admittance. Determination, results and consequences," Eng. Anal. Bound. Elem., vol. 23, no. 9, pp. 737-744, 1999.

[21] A. T. Moorhouse, "Analysis of Low Frequency Muffler based on 'Acoustic Black Hole Effect,"' no. September, 2016.

[22] W. Lapka, "Acoustic attenuation performance of selected helicoidal resonators lined with an absorbent materials of different thickness and density," Arch. Acoust., vol. 32, no. 4, pp. 247-252, 2007. 